\title{
Genetic Management of Wild and Translocated Black Rhinoceros in South Africa's KwaZulu-Natal Region
}

Rosalynn Marie Anderson-Lederer

\author{
A thesis submitted to \\ Victoria University of Wellington \\ in fulfillment for the requirement for the degree of \\ Doctor of Philosophy in Cell and Molecular Ecology
}

\section{VIt:}

Victoria University of Wellington

Te Whare Wānanga o te Ūpoko o te Ika a Mãui 


This thesis was conducted under the supervision of:

Dr. Peter A. Ritchie

Victoria University of Wellington

Wellington, New Zealand

and

Dr. Wayne L. Linklater

Victoria University of Wellington

Wellington, New Zealand 
To my parents, Louis and Linda Anderson 
Biology is a science of three dimensions. The first is the study of each species across all levels of biological organization, molecule to cell to organism to population to ecosystem. The second dimension is the diversity of all species in the biosphere. The third dimension is the history of each species in turn, comprising both its genetic evolution and the environmental change that drove the evolution. Biology, by growing in all three dimensions, is progressing toward unification and will continue to do so.

-Edward 0. Wilson

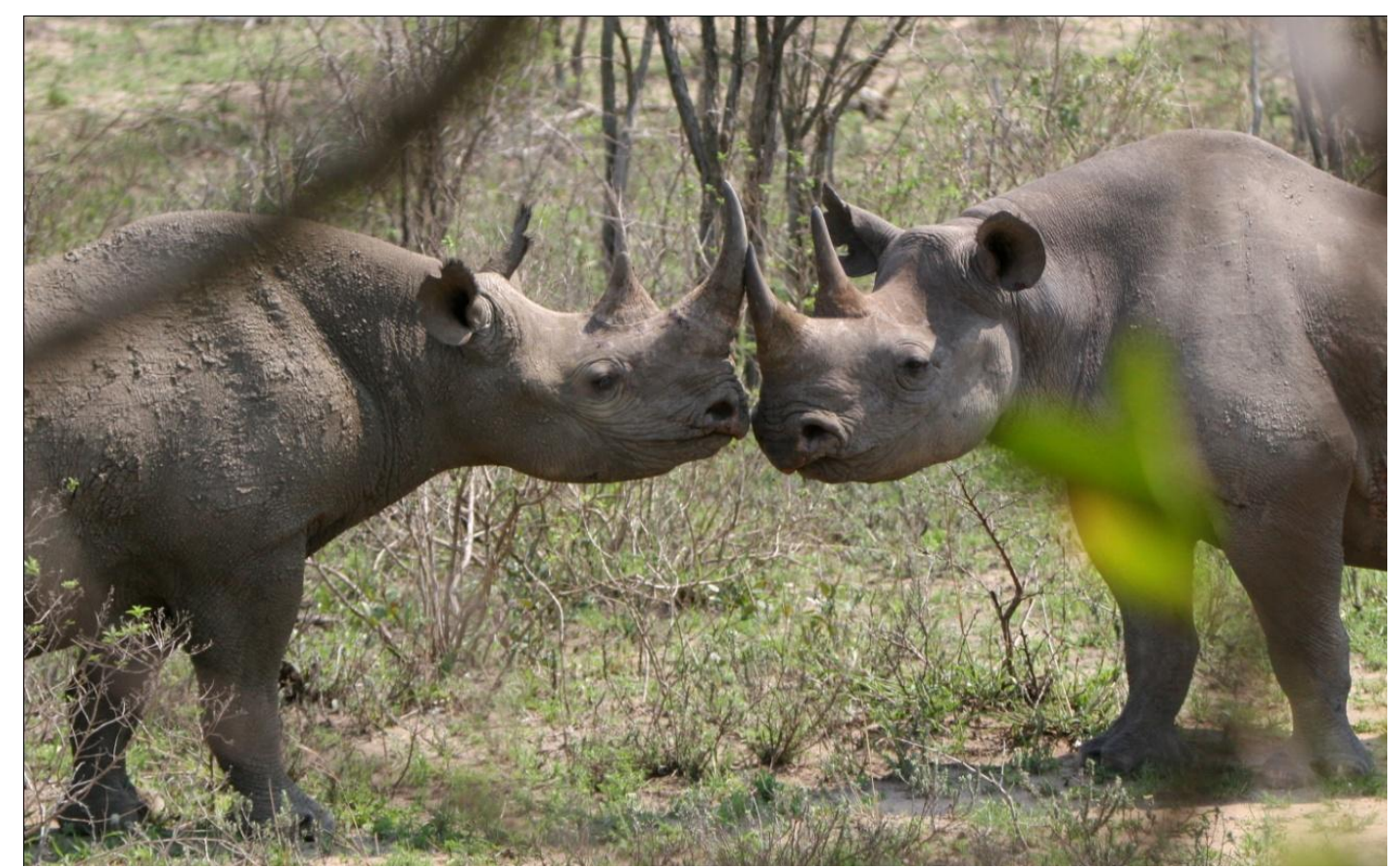

Photo by Rosalynn Anderson-Lederer 


\section{ABSTRACT}

The African black rhinoceros (Diceros bicornis) is critically endangered. Like other megafauna, the species is managed in parks and is often translocated to expand their range into reserves where they have been extirpated. Management of genetic variation has been identified as an important consideration in longterm management plans for many wild and captive endangered species including black rhino. In this thesis I examined the contemporary levels of genetic variation within the black rhinoceros (D. b. minor) in KwaZulu-Natal (KZN), South Africa, and specifically the relict source population at HluhluweiMfolozi Game Reserve (HiP), and how this information can be incorporated into management decisions to improve the long-term viability and persistence of the population. Previous studies have examined levels of genetic variation and differentiation among the three black rhino subspecies (D. b. minor, D. $b$. michaeli and D. b. bicornis) in an attempt to resolve their taxonomy and to establish baseline genetic assessments for managing populations. However, there has been a lack of genetic information based on the variable mitochondrial DNA (mtDNA) control region of the KZN metapopulation and a direct comparison of microsatellite variability between the D. b. minor populations of KZN and Zimbabwe.

The specific objectives of this study were to: (1) determine the DNA sequence of the mtDNA control region of three subspecies and estimate the level of variation within the HiP source and KZN metapopulation and compare the results with D. b. minor outside KZN and the other two subspecies; (2) use ten microsatellite DNA markers to estimate the levels of heterozygosity and 
allelic diversity in the HiP source and KZN metapopulation and compare results to previously published microsatellite data (specifically native Zimbabwe $D . b$. minor; and (3) use VoRTEX Population Viability Analysis (PVA) and HiP vital rates to model the effects of increasing population size and supplementation, and investigate what management scenarios would be most effective for minimizing the loss of genetic variation caused by genetic drift with HiP. MtDNA showed evidence of a bottleneck in the KZN D. b. minor metapopulation. The KZN metapopulation were fixed for a single haplotype $(\mathrm{n}=65)$, compared to six haplotypes $(\mathrm{n}=11)$ in native Zimbabwe D. b. minor. D. $b$. michaeli $(\mathrm{n}=21)$ samples had 13 haplotypes, while the $D$. b. bicornis $(\mathrm{n}=4)$ samples had one. Additionally, a haplotype network showed a discernable pattern of separation amongst the three subspecies with the KZN population positioned with the D. b. minor populations of Zimbabwe. While it was expected that the KZN D. b. minor would cluster together with the Zimbabwe D. b. minor because they are the same subspecies, the haplotype network provides further supporting evidence of a bottleneck in the KZN metapopulation.

The microsatellite DNA results from the KZN metapopulation also indicated a likely bottleneck pattern and possible inbreeding. The KZN metapopulation was out of Hardy-Weinberg Equilibrium, monomorphic at one locus, showed excess of homozygosity at five out of ten microsatellite DNA loci, and had 28\% less genetic variation at microsatellite DNA loci and lower allele frequency than the native Zimbabwe $D$. $b$. minor. Modified M-ratio results indicated that all three of the subspecies had been through a bottleneck. There is no pre-decline genetic information available for the KZN metapopulation, so it 
is unclear if the KZN region has historically always had low genetic variation or if the low levels were caused by a recent population decline.

No translocations are made into HiP. Modelling results of a simulated $D$. b. minor source population indicated that if no translocations into the reserve are made, expected heterozygosity $\left(H_{\mathrm{E}}\right)$ would decrease $\sim 25 \%$ over $\sim 100$ black rhino generations (BRGs). Increasing the size of the modelled population slowed the rate of loss with the mean $H_{\mathrm{E}}$ decreasing by $\sim 10 \%$ over $\sim 100$ BRGs. Models of supplementations made with a pair of black rhino (one female and one male) from the KZN metapopulation made every ten gestational years, maintained the mean $H_{\mathrm{E}}\left(H_{\mathrm{E}}=0.45\right)$ of the population over $\sim 100 \mathrm{BRGs}$, but increased $\sim 30 \%$ when supplemented with individuals from Zimbabwe. PVA results indicated that increasing gene flow through supplementation is effective and does not require a large number of individuals or need to be frequent.

If KZN has always had low levels of genetic variability, then based on the model findings, serial translocations made with KZN metapopulation rhinos into the HiP source population would be recommended to slow the rate of loss caused by drift and to maintain current levels of genetic variation. If, however, the low levels of variation were caused by a recent decline in population size, then according to the model, supplementations with native Zimbabwe $D . b$. minor would not only decrease the rate of loss of genetic variation, but would increase levels of genetic variation.

This research highlights the importance of shifting focus from increasing the number of individuals within a population to that of quality (e.g. levels of genetic variation) as black rhino move into recovery. Techniques like serial translocation and supplementation can help maintain current levels of genetic 
diversity and prevent further loss of variation in the KZN source and metapopulation, which will enable managers to improve long-term African black rhinoceros conservation efforts. These techniques can also be integrated into active management schemes for other large conservation-reliant species. More specifically, those in small, remnant populations with limited reserve or range sizes in order to increase long-term survival and population persistence. 


\section{ACKNOWLEDGEMENTS}

I want to thank all the people who played varying, although important roles in process of my $\mathrm{PhD}$.

I'm indebted to Peter Ritchie and Wayne Linklater for accepting the challenge of taking me on as a student. Thank you both very much. I could not have hand-picked better supervisors if I tried.

I am very thankful for the financial support of the following organizations: Centre for Biology and Restoration Ecology (CBRE) and U.S. Fish and Wildlife Service administered Rhinoceros and Tiger Conservation Act of 1994 (i.e. grant agreement number 96200-9-G268).

To my amazing cheerleaders: Kelly Mitchell, James Oakley, Jennifer Spoor Fishbaugh, Justine Lopez, Kathleen Julian Michalski of the USN-R, Jim and Karen Austin, Gino Grillo, Sherry Fischer and DonnaMarie Hall. The brilliant people I've met at VUW who have encouraged me and not only made me laugh, but helped me laugh at myself along the way: Jamie Tam, Jo Davy, Heidy Kikillus, Hannah Jolly, Danielle Middleton, Isle Corkery, Catherine Davis, Shalen Kumare, Lindsay Anderson, Gesine Pufal, Monica Awasthy, Andrea Varela, Danielle Hannan, Heather Constable, Sebastien Roux Paquette, Monica Gruber, Elizabeth Heeg, Anna Carter, Patricia Stein, Mary Murray, Sandra Taylor, Damon Chu, Kirsty McClure, Celine Reisser, Roan Plotz, Meagan English, Andrew Stringer and Gaius Wilson.

It's hard to eat and pay bills without a scholarship, so a huge thank you to Boris Lederer for your incredibly generous support during this project and for offering to airlift me out of South Africa when I was hospitalized. 
Family is what keeps us going when we are far from home, especially when the 'pity party for one' is in full swing, so a special thanks to my aunt Evadene Brosky for skype sessions that helped me put things into perspective. My sister-in-law Alekzandra (Allie) Lederer-Collins whose cards and care packages made me feel less homesick, and my cousins Becky Flick-Walker, Angela Flick, Melinda Brosky-Young and Larry Brosky. And most of all to my parents: Louis and Linda Anderson. Without your support through my incredibly rocky life, I'm not sure where I would have ended up. Thank you from the depths of my heart for supporting me emotionally, on occasion financially and for the numerous care packages sent from home. A thesis doesn't quite make up for not giving you those grandkids you used to always go on about, but I know you're still proud of me.

Time spent during the process of my PhD has not been easy. I lost four uncles and a cousin to cancer, one uncle to a heart attack, a mentor who I greatly respected to an age related illness, and a very dear friend of mine to a blood clot. I thank each of you for having made such a positive impact in my life. I miss you all very much. 
Acknowledgements for work by chapter:

Chapter 1: Dr's Ilse Corkery, Jo Davy and Celine Reisser for proofreading, Dr. Jacques Flamand who posed questions re: the HiP source population while working with the WWF Black Rhino Range Expansion Project. Dr. Kees Roomaaker for answering distribution and taxonomical questions.

Chapter 2: Dr. Wayne Linklater for samples collected during his post-doc. Roan Plotz, Dr.s Dave Druce and Dave Cooper for assistance while collecting field samples. Thank you to three anonymous reviewers for the Journal of African Ecology for their time and constructive comments regarding my manuscript. Raoul de Toit for answering questions regarding native Zimbabwe and translocated KZN D. b. minor populations. Dennis Kelly for answering questions about the Mkuze D. b. minor population. Dr. Oliver Ryder for answering black rhino chromosomal questions. The Terrestrial Vertebrate Group at Victoria University for proof reading.

Chapter 3: Dr. Wayne Linklater for samples collected during his post-doc. Roan Plotz, Dr.s Dave Druce and Dave Cooper for assistance while collecting field samples. Thank you to the anonymous reviewers for the Journal of Zoology for their time and constructive comments regarding my manuscript. Dr. Celine Reisser for proofreading. Dr.s Andrea Vilera and Kristina Ramstand for discussions involving genetics software. Keryn Adcock for answering questions re: South African game reserve rhino $/ \mathrm{km}^{2}$. Dr. Richard Emslie for answering questions about black rhino abundance.

Chapter 4: Geoff Clinning for HiP population information. Keryn Adcock for answering questions regarding rhino $/ \mathrm{km}^{2}$ and black rhino resource questions. James Oakley for assistance with Vortex data input code. Dr.s Robert Lacy and Carlo Pacioni for answering Vortex parameter questions. Linklater lab group for discussions regarding Vortex.

Chapter 5: Hannah Jolly and Dr. Heidy Kikillus for thesis proofreading. Dr.s Jamie Tam, Catherine Davis and Danielle Middleton for thesis formatting assistance. 


\section{Table of Contents}

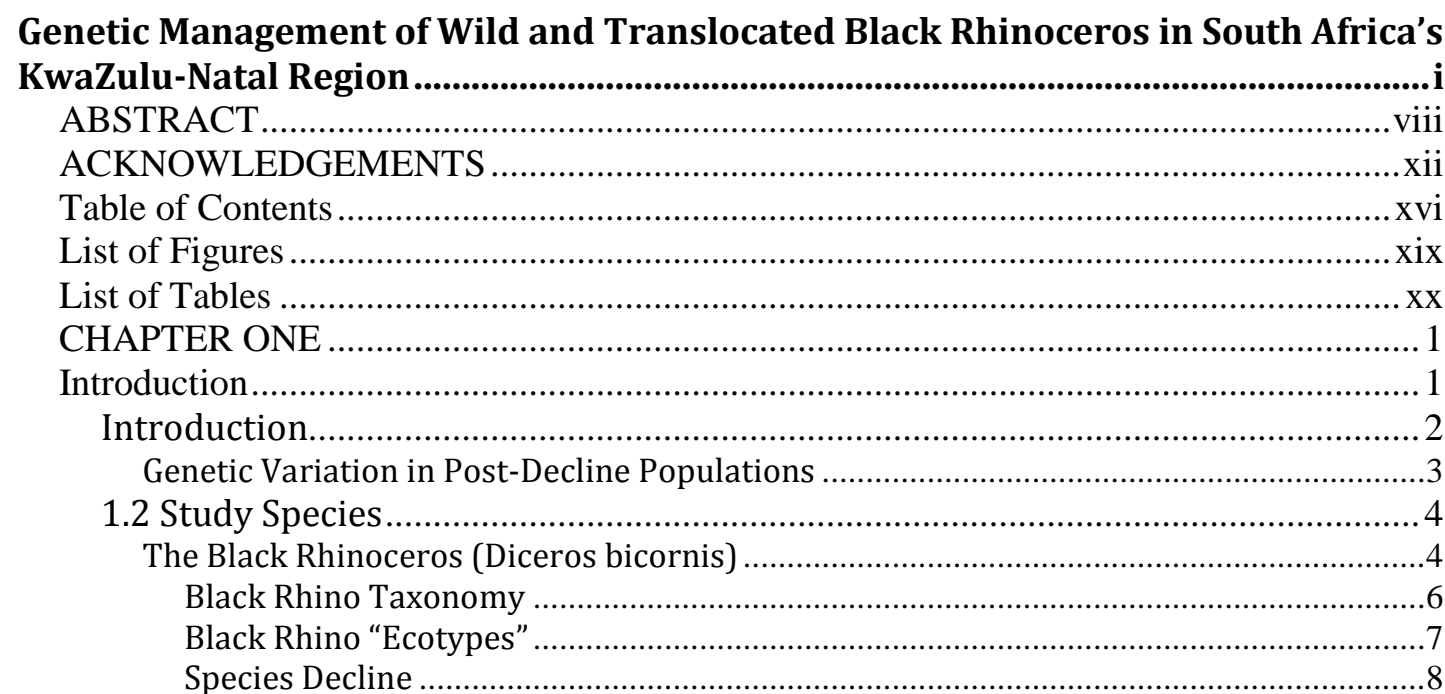

1.3 Hluhluwe-iMfolozi Game Park (HiP), Source Population ................................... 12

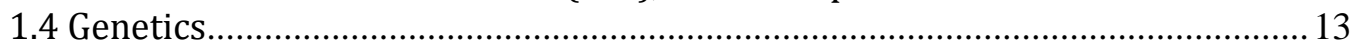

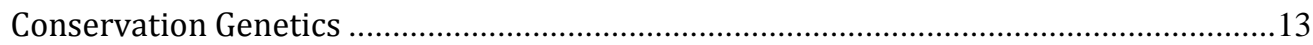

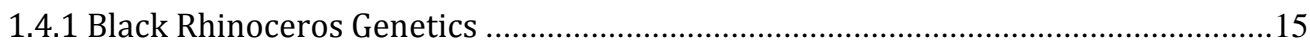

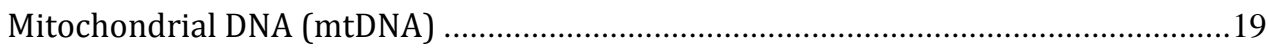

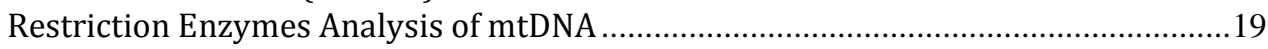

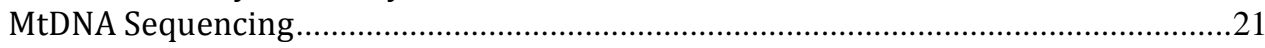

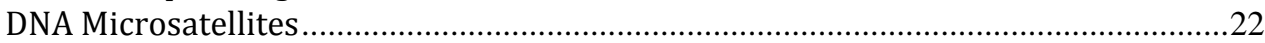

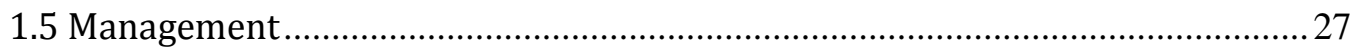

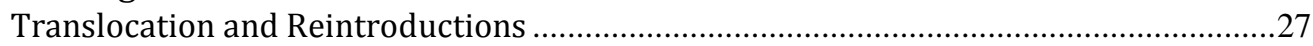

The Need for a Paradigm Shift in Rhino Conservation Practice ........................................29

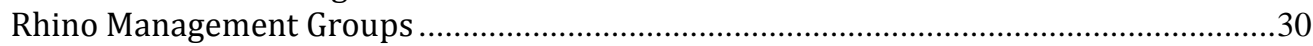

The International Union for Conservation of Nature (IUCN) .............................................31

Regional Rhino Conservation Groups Affiliated with the IUCN …………………..............31

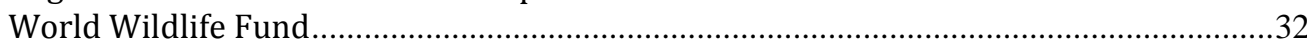

1.6 Recommended Guidelines for Black Rhino Management.....................................32

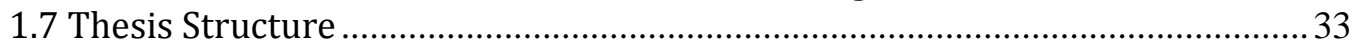

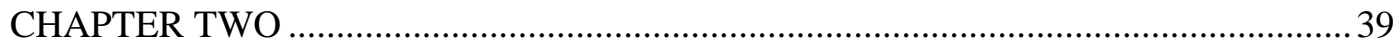

Limited mitochondrial DNA variation within South Africa's black rhino (D. b. minor)

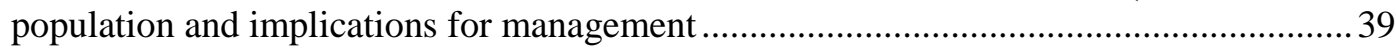

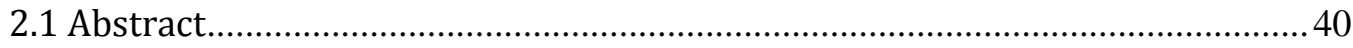

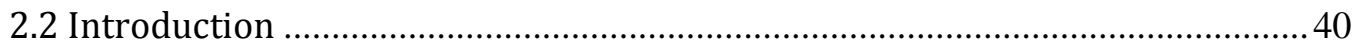

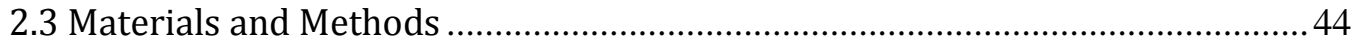

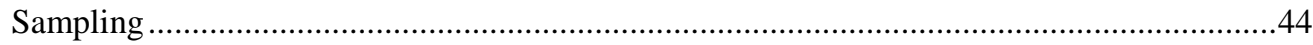

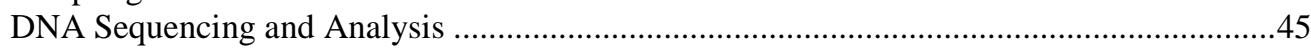

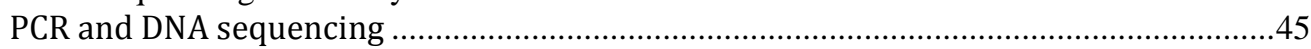

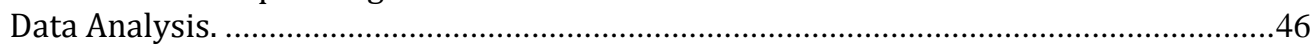

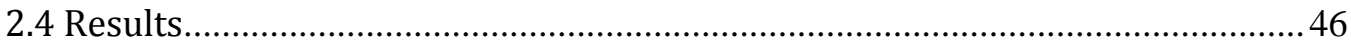

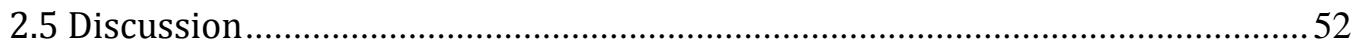

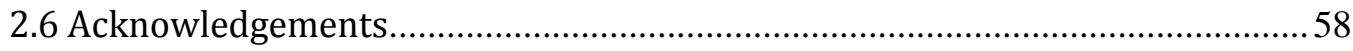

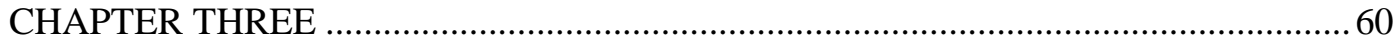

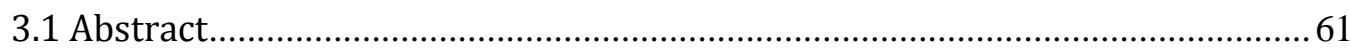

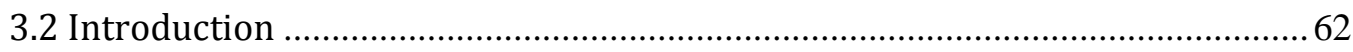

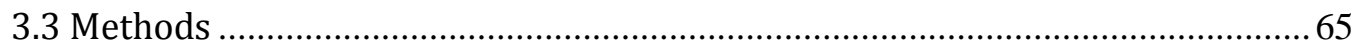

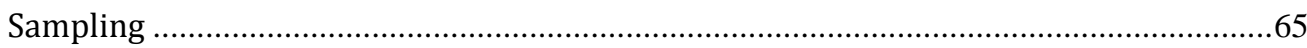

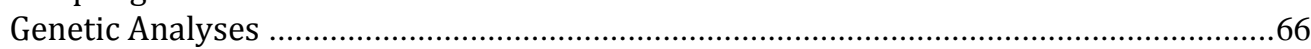

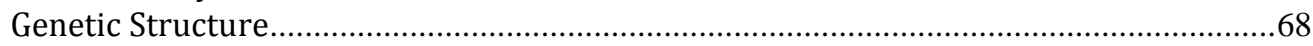

Testing for a Genetic Bottleneck ……………………................................................69 


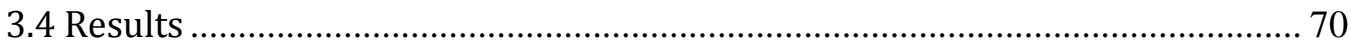

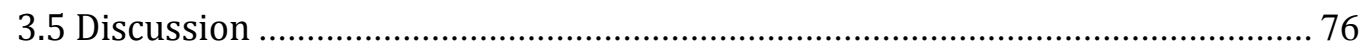

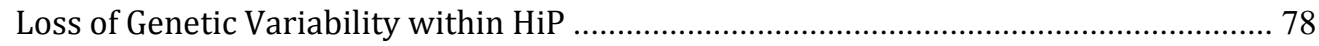

Population Increases through Land Acquisitions and Serial Translocations .................. 79

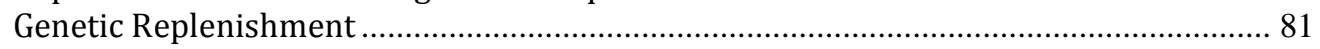

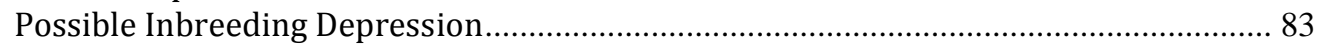

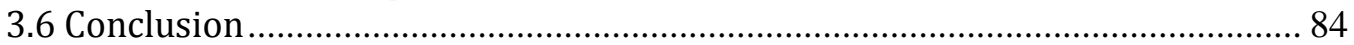

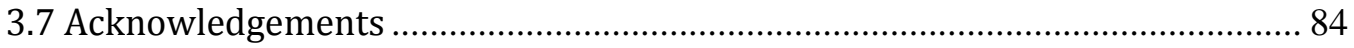

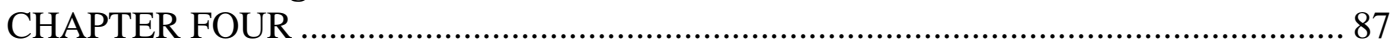

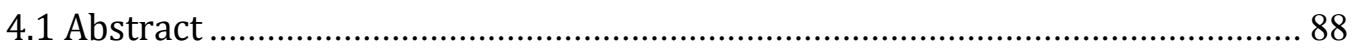

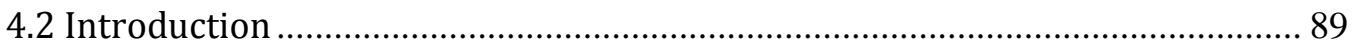

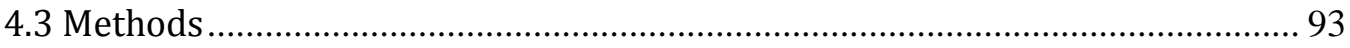

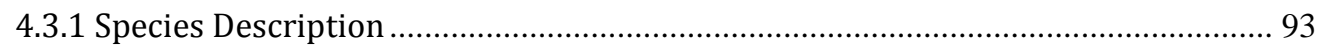

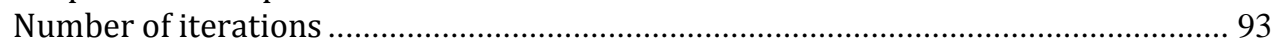

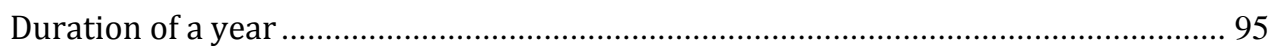

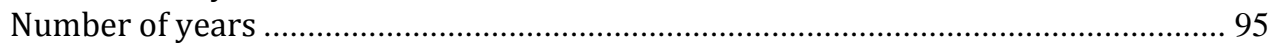

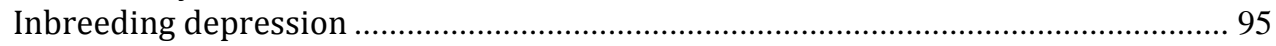

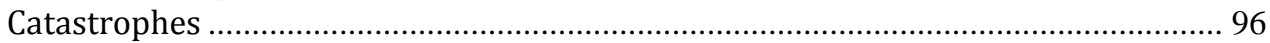

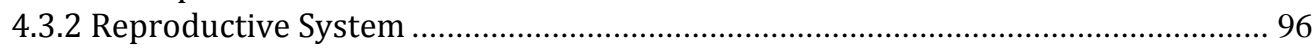

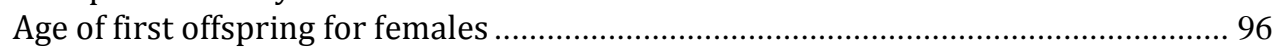

Age of first offspring for males .................................................................................. 97

Maximum age of reproduction, number of progeny per year ...................................... 97

Sex ratio at birth - in \% male................................................................................. 97

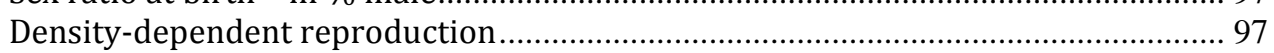

\% Adult Females Breeding ……………...................................................................... 98

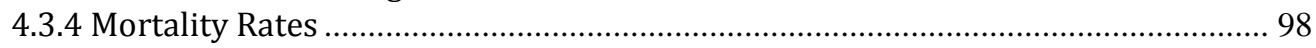

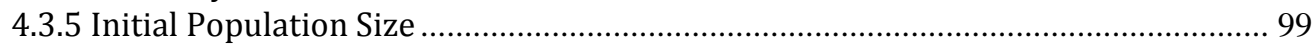

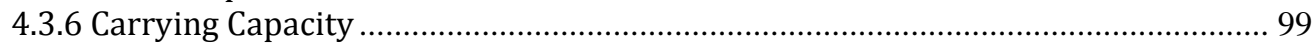

4.3.7 Harvest (Capturing individuals to relocate to other reserves) ............................... 100

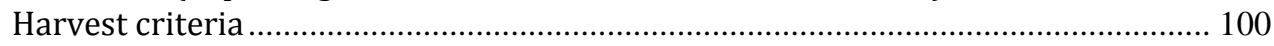

Optional criteria for harvest ..................................................................................... 100

Number of female and male of each age to be harvested .......................................... 100

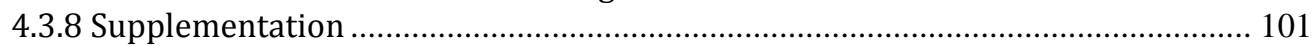

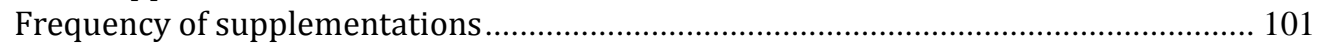

Number of females and males supplemented ………………................................ 101

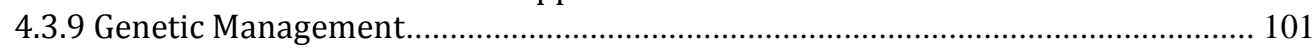

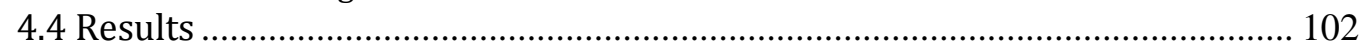

4.4.1 Levels of Genetic Variation with No Supplementation......................................... 102

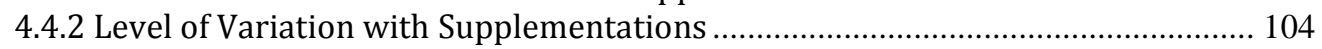

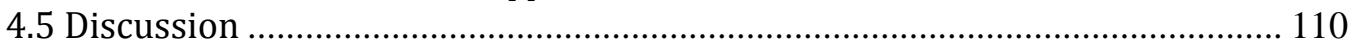

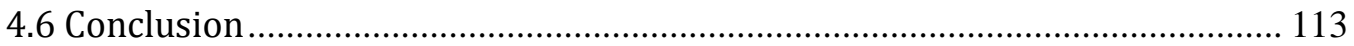

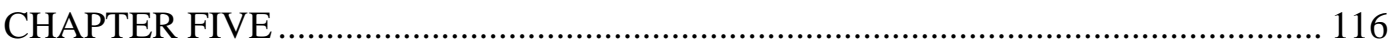

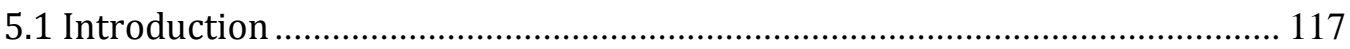

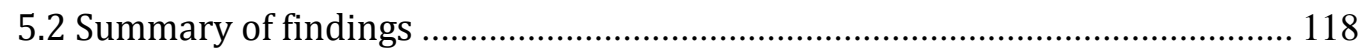

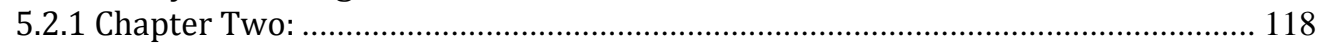

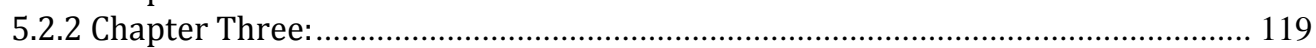

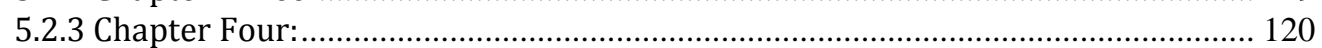

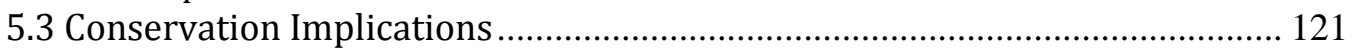

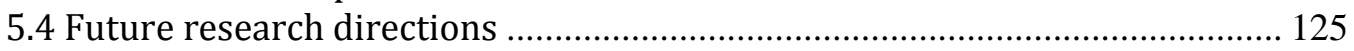

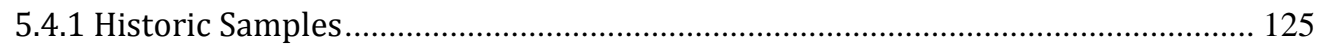

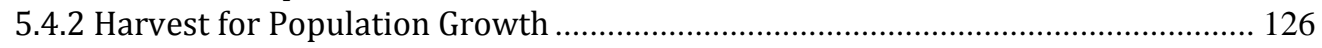

5.4.3 Translocation Cohort Sizes and Composition ..................................................... 127

5.4.4 Functionally important genetic variation and Conservation Genomics ................ 128

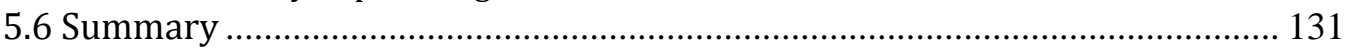

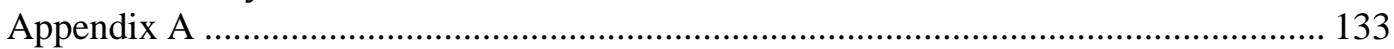

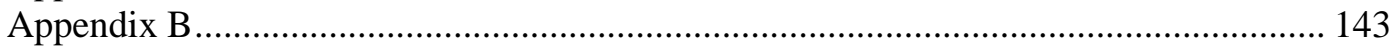

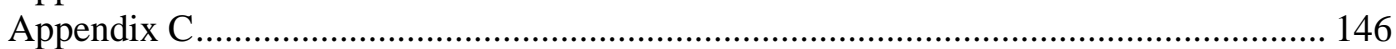


5.7 References .

148

xviii 


\section{List of Figures}

Figure 1.1: Previous 'pre-1900' distribution map of African black rhino 9

Figure 1.2: Revised 'pre-1900' distribution map of black rhino in west Africa..10

Figure 1.3: Black rhino game reserves in KwaZulu-Natal 10

Figure 2.1: Map of Southern Africa showing black rhinoceros sample sites......42

Figure 2.2: Statistical parsimony haplotype network. .50

Figure 3.1: Map of southern Africa with sample sites. 63

Figure 3.2: STRUCTURE population genetic structure output for D. bicornis......74

Figure 3.3: Black rhino game reserves in KwaZulu-Natal 80

Figure 4.1: The mean population sizes for a modelled population with a carrying capacity of 430 , with and without inbreeding depression, and a $4 \%$ harvesting rate per gestational year. 103

Figures 4.2: The mean population sizes for a modelled population with a carrying capacity of 430 , with and without inbreeding depression, with a $4 \%$ harvesting rate, with and without supplementions 106

Figure 4.3: Mean expected heterozygosity for a modelled population with a carrying capacity of 430, without inbreeding depression, with a $4 \%$ harvesting rate per gestational year with various supllementation regimes with one female and one male. 107

Figure 4.4: Mean expected heterozygosity for a modelled population with a carrying capacity of 430 , without inbreeding depression, with a $4 \%$ harvesting rate per gestational year with various supplementation regimes with one female and one male, and two females and two males. 108

Figure 5.1: The five structures of fragmented populations. 127

Figure 5.2: A diagram of interacting factors in the conservation of a natural population 


\section{List of Tables}

Table 1.1: Translocation history of D. B. minor from HiP from $1962-2008 \ldots . . .13$

Table 1.2: Genetic studies that include black rhino .17

Table 2.1: Rhinoceros subspecies and sources analyzed for mitochondrial DNA variation . .47

Table 2.2: Mitochondrial DNA D-loop sequence variability within subspecie...48

Table 2.3: Summary statistics for the mitochondrial control region sequence variability in each subspecies and haplotype identifiers used in Figure 2.2.......49

Table 2.4: Microsatellite results from Harley et al. 2005 and Karsten et al. 2011

Table 3.1: Characteristics of 10 microsatellite markers used to type 127 black rhinoceros and number of alleles found in 118 KwaZulu-Natal D. b. minor........64

Table 3.2: Subspecies of Diceros bicornis and corresponding populations and sample size. .65

Table 3.3: $H_{\mathrm{e}}$ and $H_{\mathrm{o}}$ per locus, per subspecies with associcated P-values...69

Table 3.4: A) Pairwise $F_{\mathrm{ST}}$ values B) Pairwise $R_{\mathrm{ST}}$ values C) Pairwise $D_{\text {est }}$ values for D. bicornis. . .71

Table 3.5: Microsatellite results from this study, Harley et al. 2005, Karsten et al. 2011, Muya et al. 2011 and Van Coeverden de Groot et al. 2011

Table 4.1: Vortex scenario parameters .94

Table 4.2: Allele frequencies for the 10 microsatellite DNA loci examined in Chapter 3 for HiP D. b. minor 102

Table 4.3: Allele frequencies for the 10 microsatellite DNA loci examined by Garnier et al. (2001) for native Zimbabwe D. b. minor 102

Table 4.4: Expected and observed heterozygosities with standard error for $D . b$. minor with and without inbreeding depression at 430 carrying capacity for years $50,100,150,200,250,500,700$ and 1000 104

Table 4.5: Expected and observed heterozygosities with standard error for $D . b$. minor with and without inbreeding depression at 830 carrying capacity for years $50,100,150,200,250,500,700$ and 1000 104

Table 4.6: Expected and observed heterozygosities with standard error for a population of D. b. minor with and without inbreeding depression at 430 carrying capacity for years 50,100, 150, 200, 250, 500, 700 and 1000 and a 4\% harvest. 
Table A.1: Expected heterozygosities with standard error; with and without inbreeding at 430 carrying capacity for years 50, 100, 200, 250, 500, 700 and 1000; with mean population sizes and standard error....................145

Table A.2: Probability of success or extinction for founder populations with sizes from 5-30 individuals. 


\section{CHAPTER ONE}

\section{Introduction}

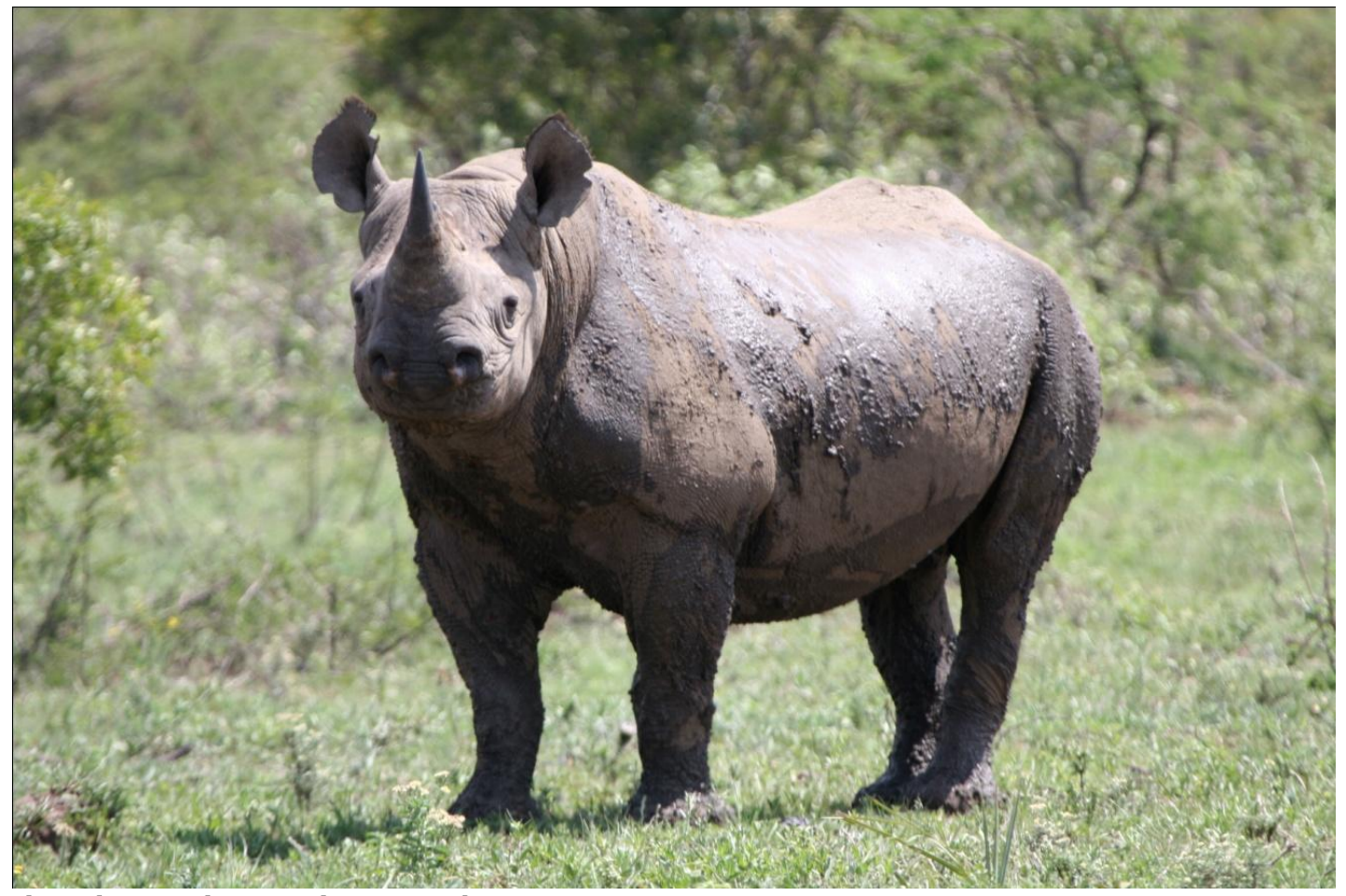

Photo by Rosalynn Anderson-Lederer 


\section{Introduction}

The evolutionary process is dependent on genetic variation in order for populations to adapt to changes occurring in the environment (e.g. diseases, predators, climate change) (Lacy 1987b). Large breeding populations are able to maintain high levels of variation and evolutionary potential (Lacy 1987b; Bijlsma et al. 2000), but small populations are prone to decreases in the level of genetic variation and inbreeding depression. This increases the risk of extinction and reduces their evolutionary potential (Lacy 1987b; Frankham et al. 1999; Garner et al. 2005; Frankham 2005). It is unclear which, or how many, traits will be affected by inbreeding depression, or how long before adverse genetic effects manifest in small populations (Hogg et al. 2006). However, when genetic diversity is lost in small populations, the rate at which a population recovers is primarily contingent on mutation rate (Lynch 1996), which can take a considerable number of generations (Allendorf and Leary 1986a).

Many African wild animal populations have gone through gradual or sudden population declines for reasons that include overexploitation, habitat loss and disease (e.g. rinderpest) (Western and Vigne 1985; Simonsen et al. 1998; Harley et al. 2005). Historic population sizes and declines, however, are seldom well documented. Europeans recorded wildlife sightings while exploring and hunting the continent, but documentation was general with basic descriptions, usually kept in personal journals (Tingley and Beissinger 2009). These records are valuable for estimating historic ranges, but their use is limited when estimating historic population sizes and declines (Shoo et al. 2006; Rookmaaker 2007; Tingley and Beissinger 2009). It was often only in extreme 
cases of species decline that detailed population information was recorded and is available (Shaffer et al. 1998).

Genetic Variation in Post-Decline Populations

Many threatened or endangered species that have experienced population declines are now conservation-reliant (see: Miller et al. 1988; Walters 1991; Tyus and Saunders 2000; Jamieson et al. 2006; Johnson et al. 2010). The level of genetic variation remaining after a severe population decline is determined by past and current population sizes, and pre-decline levels of diversity (Frankham et al. 2002). An ongoing loss of diversity is expected if population recovery is slow (Nei et al. 1975).

To determine loss of genetic variation as a consequence of decline, an assessment of pre-decline gene flow and variability is required, but seldom possible (Briskie and Mackintosh 2004). Museum samples can be used to apprise missing data (greater prairie chicken (Tympanuchus cupido) Bouzat et al. 1998; whooping crane (Grus americana) Glen et al. 1999), but when postdecline levels of genetic variation are low and no museum specimens are available it may be difficult to determine whether the low variability was due to the reduction in population size or a general response to demographic and environmental differences (Bouzat et al. 1998).

If pre-decline levels of genetic variation are unknown, post-decline population levels should still be sampled and monitored. The collected data will establish baseline levels of variation, assisting in future reintroductions and parentage analyses in founder populations (Schwartz et al. 2006). 


\subsection{Study Species}

The Rhinocerotidae (meaning "nose horns") are the second largest living land animals after elephants (Kingdon 1997). They are in the order Perissodactyla (odd-toed ungulates), which includes the Tapiridae (Tapirs) and Equidae (Horses) (Silberman and Fulton 1979; Lacombat 2005). First appearing approximately 56 to 34 million years ago (MYA), Rhinocerotidae included some 26 different genera in Eurasia spreading to North America and later, in the Miocene Epoch (approximately 23 to 5 MYA), from Asia into Africa (Lacombat 2005). The earliest genera of Rhinocerotidae went extinct during early Oligocene (37 MYA), and Rhinocerotidae declines at the end of the Miocene are attributed to climate change (Lacombat 2005). Today there are five extant rhinoceros species: three Asian; Sumatran (Dicerorhinus sumatrensis; critically endangered A2abd; C1+2a(i) ver 3.1 (van Strien et al. 2008a)), Javan (Rhinoceros sondaicus; critically endangered C2a(i); D ver 3.1 (van Strien et al. 2008b)) and Indian (Rhinoceros unicornis; vulnerable B1ab(iii) (Talukdar et al. 2008)) and two African; black (Diceros bicornis) and white (Ceratotherium simum; near threatened ver 3.1 (Emslie 2012)) (Owen-Smith 1988).

\section{The Black Rhinoceros (Diceros bicornis)}

Unlike their Asian counterparts, the African black rhino lack incisors and canine teeth (Kingdon 1997; Emslie and Brooks 1999). Their brachyodont teeth (low crown) enable them to browse on coarse plant material like leaves, twigs, branches and long grass (Kingdon 1997; Lacombat 2005). They have two horns which have a dermal origin and are comprised of laminated keratinaceous filaments (compressed hair and fingernail matter) that grow throughout the 
animal's life (Silberman and Fulton 1979; Lacombat 2005). Black rhino have a muscular finger-like prehensile lip used for browsing (Skinner and Smithers 1990). They range in height from $1.4-1.8 \mathrm{~m}$ (55 - 71 in.) at the shoulder and vary in weight from 1000 - $1800 \mathrm{~kg}$ (2200 - $3970 \mathrm{lbs})$; their body length ranges from 2.9 - 3.75 m (114 - 148 in.) (Kingdon 1997; Emslie and Brooks 1999).

Black rhino are not actually black, but generally grey; they may appear to vary in colour as a result of mud or dust bathing (Kingdon 1997). Wallowing may help reduce body temperature and protects rhino from ectoparasites (e.g. ticks and biting flies). Distinguishing the sex of a rhinoceros in the field can be difficult because males have undescended testes and, therefore, lack a scrotum (Kingdon 1997). The genitalia of both sexes face backwards and they are capable of projecting urine up to three to four meters (Schenkel and SchenkelHulliger 1969). Black rhino tend to be asocial and while female home ranges overlap, males tend to live in mutually exclusive home ranges (Owen-Smith 1988; Conway and Goodman 1989). Both sexes can live up to $30-35$ years in the wild, but that is extended to $45+$ years in captivity (Owen-Smith 1988). One black rhino generation is approximately 14 years (Brooks and Adcock 1997). Gestation is approximately 15.33 months (Schenkel and SchenkelHulliger 1969; Owen-Smith 1988; Bertschinger 1994) with infants weighing between $27-45 \mathrm{~kg}(60-100 \mathrm{lbs})$ at birth. Females give birth to a single offspring that is able to stand and walk shortly after birth. They start to suckle within 3 - 4 hours of birth and are able start eating solid food (grass and nonwoody plants) within 10 days (Schenkel and Schenkel-Hulliger 1969; OwenSmith 1988; Bertschinger 1994). The mean intercalving time was shown to be between 30 and 44 months within HiP (Bertschinger 1994). 


\section{Black Rhino Taxonomy}

Before wildlife managers can design effective conservation plans they must first resolve and understand the target species' taxonomy. Identification inaccuracies can lead to inadequate protection for some species resulting in extinction and/or the possibility of unwanted hybridization (e.g. black wildebeest (Connochaetes gnou) Grobler et al. 2011) of sister taxon when translocating animals for supplementation or reintroductions (Allendorf and Luikart 2007).

Taxonomic resolution includes two processes: (1) listing and priority setting involving legislation; and (2) recovery planning and in situ and ex situ conservation actions (Mace 2004). Once a species has been listed, conservation managers can turn their attention to figuring out why the species is in decline and implementing strategies for arresting and mitigating the effects of the decline (Mace 2004).

Zukowsky (1965) described 17 separate black rhino subspecies based on photos, literature, museum skull specimens and zoo animals. Groves' (1967) study of a smaller number of skulls narrowed the number of subspecies down to seven, based on size and morphology. However, subsequent but unpublished data collected on southern Africa black rhino skulls claim to refute Groves' findings (du Toit 1987). Groves and Grubb (2011) have increased the subspecies number up to eight to include Diceros bicornis bicornis (Linnaeus, 1758; from the Cape north to Kuruman, South Africa to southern Namibia); D. $b$. chobiensis, (Zukowsky 1965; the Okavango region of Botswana) D. b. minor (Drummond, 1976; KZN, South African north to north-west Tanzania and the south-west borders of Kenya); D. b. occidentalis (Zukowsky, 1922; northern Namibia and southern Angola); D. b. michaeli (Zukowsky 1965; north-west 
Tanzania into eastern Kenya); D. b. brucii (Lesson, 1842; Somalia, western Somalia and northern Sudan); D. b. ladoensis (Groves 1967; Kenya Rift Valley north-west into southern Sudan); and D. b. longipes (Zukowsky 1949; southwestern Chad, northern Cameroon and north-east Nigeria).

\section{Black Rhino "Ecotypes"}

In the context of a poorly resolved black rhino taxonomy, a meeting of rhino managers in 1986 proposed that for better metapopulation management, formal recognition of vernacular "ecotypes" be accepted and applied (Rookmaaker 1995, 2005, 2011). In 1990, taxonomic accuracy was set aside for pragmatic reasons and ecotype designations were officially recognized (Rookmaaker 2005; du Toit 2006a). Even though more than one subspecies occupied each ecotype region before the decline in black rhino numbers, in 1990 there was only one subspecies remaining in each of the regions. The three remaining recognized ecotypes and the corresponding subspecific names of subspecies remaining within the ecotypes (Eastern (D.b. michaeli): Ethiopia, Kenya, Rwanda, Somalia, Sudan, Tanzania, Uganda; South-western (D. $b$. bicornis): Angola, Botswana, Namibia, South Africa; and South-central (D. $b$. minor): Angola, Botswana, Congo Malawi, Mozambique, South Africa, Swaziland, Tanzania, Zambia, Zimbabwe) are now used interchangeably.

The critically endangered A2abcd ver 3.1 (Emslie 2011) D. b. michaeli (Eastern) is mostly found in Kenya, although small numbers have been accounted for in Rwanda and Tanzania. There is an extralimital or 'insurance' population in South Africa that was founded with individuals from Kenya. This subspecies is, however, probably nationally extinct in Ethiopia (Amin et al. 
2006; Emslie and Brooks 1999). The vulnerable D1 ver 3.1 (Emslie 2011) D. $b$. bicornis (South-western) are found in Namibia and parts of South Africa, and are presumed nationally extinct in Angola and Botswana (Emslie and Brooks 1999). The critically endangered D. b. minor A2abcd ver 3.1 (Emslie 2011) (Southcentral) are mainly found in South Africa with smaller numbers in Zimbabwe (native), Tanzania (native), Swaziland (reintroduced), Malawi (reintroduced), Zambia (reintroduced), and Botswana (reintroduced). They are believed to be extinct in Angola and Mozambique (Amin et al. 2006). The last sighting of five remaining members of a fourth subspecies D. b. longipes (Western 'ecotype') was in 2001 in Northern Cameroon. This ecotype was declared 'probably extinct' in 2006 and subsequently declared 'extinct' in 2011 (Emslie 2011). While each of the ecotypes occupy different regions in Africa, there are no impervious geographical boundaries between them (Emslie and Brooks 1999). With the three remaining subspecies already red-listed, managers are addressing the second taxonomic process of how best to manage each subspecies.

\section{Species Decline}

Black rhino once numbered in the hundreds of thousands but they have suffered an extraordinary decline in the last century, disappearing more quickly than any other large mammal (Hitchins 1975; Western and Vigne 1985; Ashley et al. 1990; Swart et al. 1994; Harley et al. 2005). Human hunting is the primary reason for their decline (Western and Vigne 1985; Emslie and Brooks 1999; Harley et al. 2005). Black rhino were (and continue to be) killed for trophies, 
meat, hides (for shields and good luck charms) and for their horns (traditional medicines and handles for daggers).

Prior to 2004, it was assumed that historic black rhino distribution extended from the Cape of South Africa, north to Somalia and Ethiopia, west to Senegal and Guinea (Figure 1.1). Rookmaaker (2004) revised the extent of

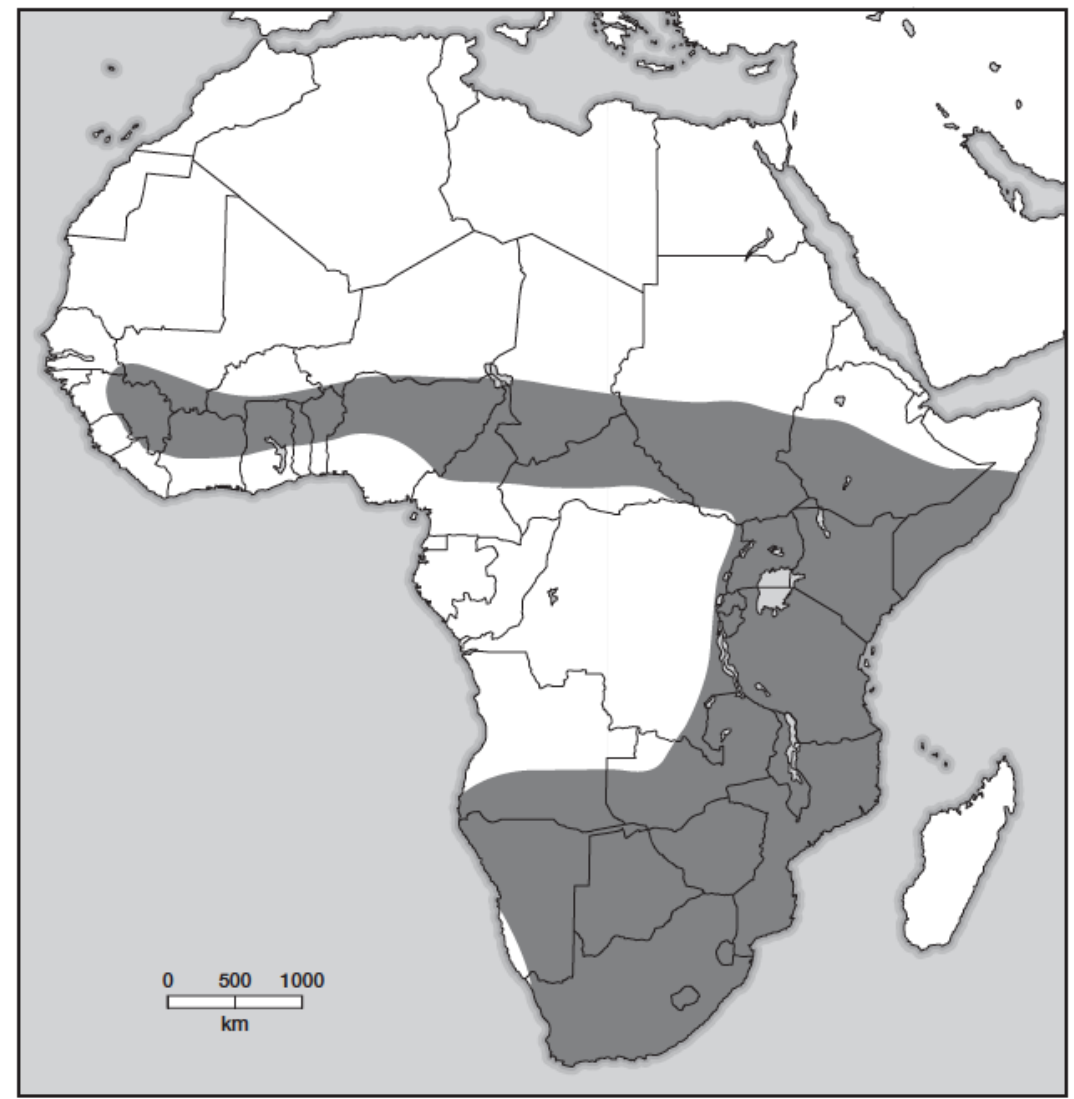

Figure 1.1: Previous 'pre-1900' distribution map of African black rhino. (Map from Emslie and Brooks 1999)

historic distribution in West Africa (only to as far as Nigeria, possibly the southwest of Niger) based on a study of bibliographical and iconographic literature (Figure 1.2). Revising the extent of the black rhino historic range to the west may help some aspects of taxonomic resolution. 


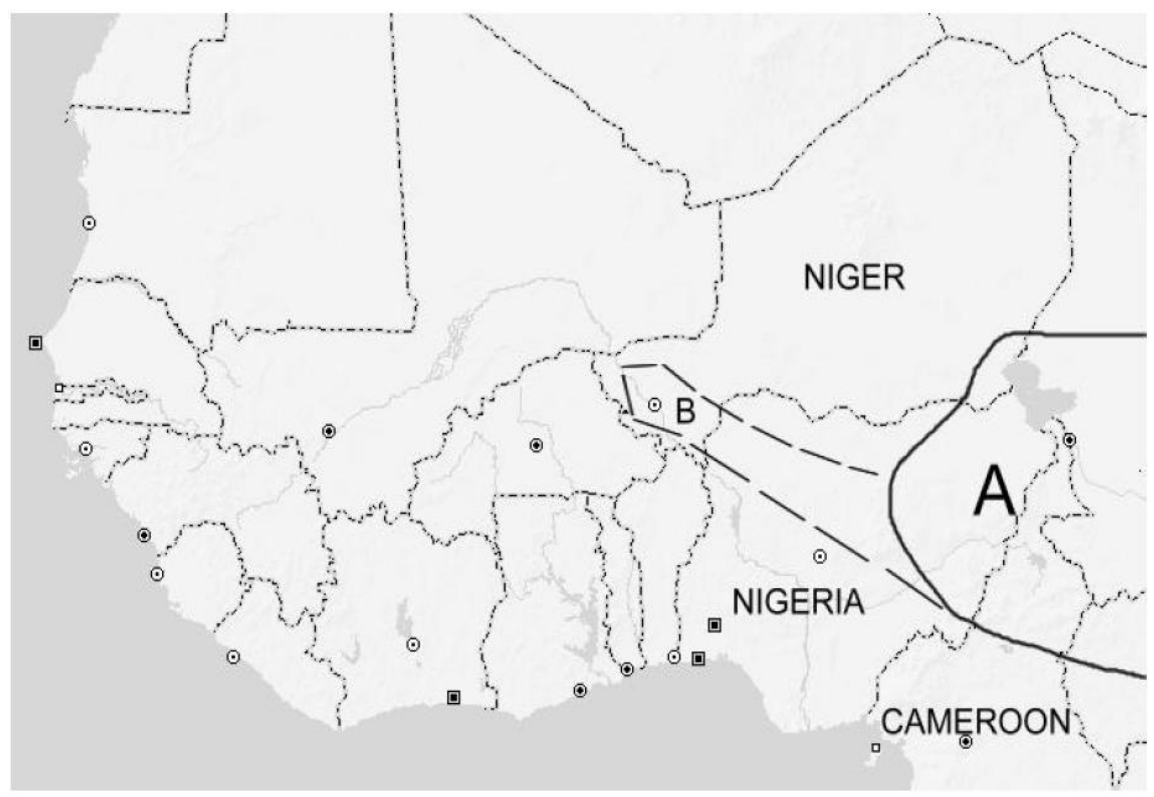

Figure 1.2: Revised 'pre-1900' distribution map of black rhino in West Africa. Area A is the likely extent of the black rhino to the west. Area B is the maximum possible extent of the range. (Map from Rookmaaker, 2004)

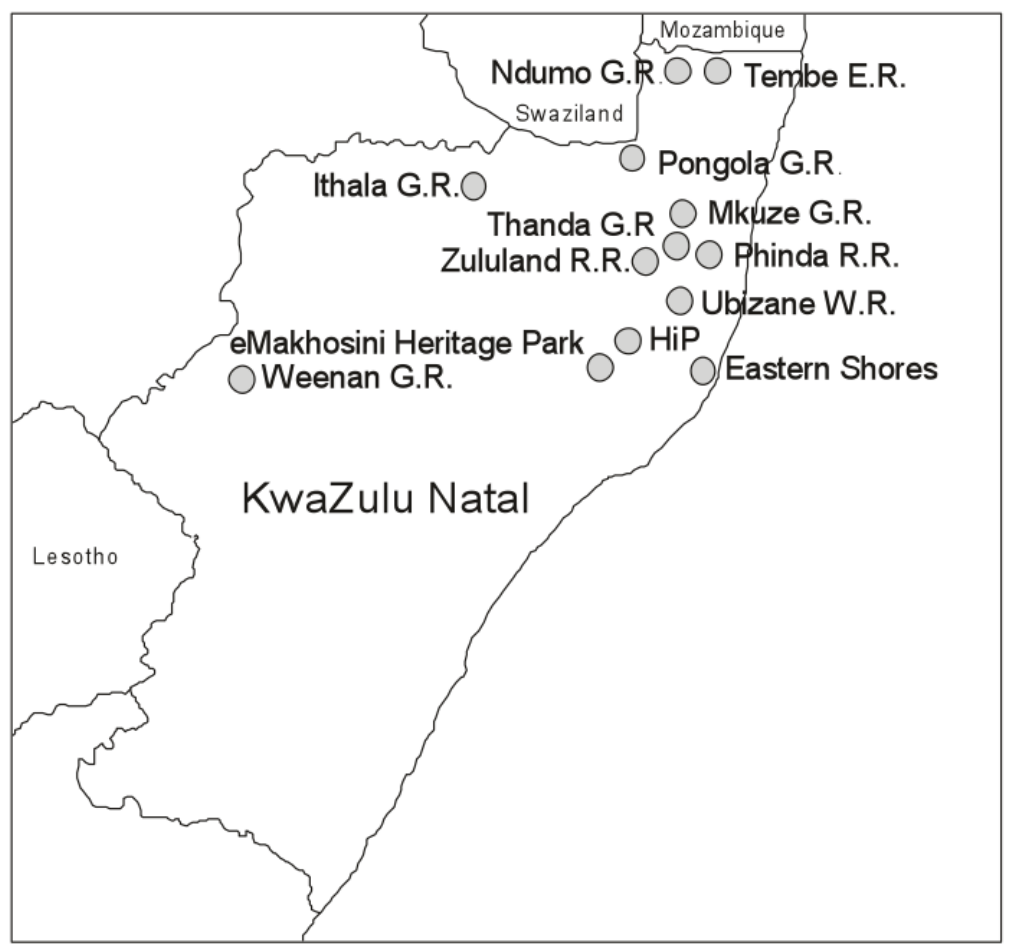

Figure 1.3: Black rhino game reserves in KZN. Ndumo Game Reserve, Tembe Elephant Reserve, Pongola Game Reserve, Ithala Game Reserve, Mkuze Game Reserve, Thanda Game Reserve, Phinda Resource Reserve, Zululand Rhino Reserve, Ubizane Wildlife Reserve, eMakhosini Heritage Park, HiP, Eastern Shores, Weenan Game Reserve 
From 1970 to 1995, black rhino numbers were reduced from approximately 65,000 to an estimated 2,400 (Emslie et al. 2009). However, by the end of 2010, in situ conservation methods increased the total number of black rhino to 4,880 (Emslie 2011). The number of D. b. minor in South Africa increased from a mere 110 reported in 1930 to 1,684 by the end of 2010 (Emslie 2011). While the number of D. b. minor in South Africa appears to be increasing, a recent spike in poaching has decreased the number of $D . b$. minor in Zimabwe to approximately 431 (Emslie 2011).

D. b. minor were extirpated from Kruger National Park (KNP), South Africa in the 1930's, but have been reintroduced through translocations from South Africa and Zimbabwe. Ferreira et al. (2011) estimated that there were, approximately 627 D. b. minor in KNP, which would make it the largest $D . b$. minor population in Africa. Their results were based on block surveys consisting of 155 individual black rhino visual encounters (Ferreira et al. 2011). Information gained by estimating population sizes is valuable and is often required to justify the implementation of mangement schemes (Tacha et al. 1982), but as management budgets allow, more precise methods of determining population size should be utilized.

Hluhluwe-iMfolozi Game Park (HiP), in the KwaZulu-Natal Region of South Africa used estmates for mangement purposes from 1998 to 2008 only to discover that their estimates were inflated by nearly 50\% (Clinning et al. 2009). To improve accuracy of $D . b$. minor numbers, HiP currently employs a Priority Species Monitor (specialized ranger), who spends up to three months in each of it's five sections visually locating as many black rhino as possible. Recent monitoring put the number of D. b. minor within HiP at roughly 220 (Clinning et 
al. 2009) or $13 \%$ of the total D. b. minor in South Africa and approximately half of the D. b. minor in KwaZulu-Natal (Emslie 2011). HiP is the focal site for this project (Figure 1.3).

\subsection{Hluhluwe-iMfolozi Game Park (HiP), Source Population}

$\mathrm{HiP}$ is home to the largest relict population of the critically endangered $D . b$ minor. The park covers $\sim 96,000$ hectares (ha) and consists of two reserves, Hluhluwe and iMfolozi, which were previously managed separately but are now managed as a single game park with a connecting corridor between the two reserves. HiP is further broken down into five administration sections: Makhamisa (Wilderness-southernmost), Mbhuzane (South-west), Masinda (south-central), Nqumeni (north-central) and Manzibomvu (north). Field rangers regularly patrol each section and a game-proof fence surrounds the entire park. Located in the KwaZulu-Natal (KZN) region, it was one of the first game parks established in South Africa. Before becoming a game park in 1897, HiP was used as a hunting ground for King Shaka kaSenzangakhona, founder of the Zulu Nation (Brooks 2000). The park is also home to many other extant African wildlife species, including all of the "Big Five" (buffalo, elephant, lion, leopard and rhino) and has been a major source population for D. b. minor since the early 1960's (Table 1.1).

Along with its historical significance, HiP is instrumental in the conservation of important southern African species. Southern white rhino (Ceratotherium simum simum), once found in large numbers across southern Africa, had been hunted to near extinction (Emslie and Brooks 1999). By the end of the $19^{\text {th }}$ century 200 individuals remained in South Africa; most were found 
in the iMfolozi section of HiP (Rookmaaker 2000). Protection was set up for them and through ex situ breeding programmes their numbers grew. As of December 2010, there were $\sim 20,170$ white rhino in the wild (Emslie 2011).

Table 1.1: Translocation history of D. b. minor from HiP from 1962 - 2008. (Adapted from Hitchens 1984, Hall-Martin \& Knight 1994 and Brooks \& Adcock 1997)

\begin{tabular}{lcc}
\hline Game Park & Year(s) & Number \\
\hline Eastern Shores & 1984 & 7 \\
Elandsdrift & 1994 & 5 \\
Great Fish River & $1989-1991$ & 10 \\
Ithala Game Reserve & $1977-1984$ & 17 \\
Kruger National Park & $1971-1982$ & 55 \\
Lapalala Game Reserve & $1990-1993$ & 11 \\
Marakele National Park & $1993-2002$ & 2 \\
Maremani Nature Reserve & 2003 & 5 \\
Mauricedale Game Ranch & 2007 & 1 \\
Malilangwe Wildlife Reserve & 1998 & 27 \\
Ndumo Game Reserve & $1962-2008$ & 18 \\
Phinda Game Reserve & $2004-2008$ & 9 \\
Phongola Game Reserve & 2006 & 5 \\
Pilanesberg Game Reserve & $1981-1983$ & 18 \\
Rhinolands & 1995 & 6 \\
Sable Game Reserve & 1991 & 4 \\
Shamwari Game Reserve & 1993 & 4 \\
Somkhanda Game Reserve & 2007 & 8 \\
Sutton Game Reserve & 2006 & 3 \\
Tembe National Elephant Park & 1994 & 13 \\
Weenen Game Reserve & $1983-2007$ & 3 \\
Western Shores & 2003 & 4 \\
Zulu Nyala Game Reserve & 2004 & 1 \\
Zululand Game Reserve & 2005 & 18 \\
& &
\end{tabular}

\subsection{Genetics}

\section{Conservation Genetics}

Anthropogenic activities are the primary reason for species decline and extinction (e.g. habitat destruction and overexploitation) (Caughley 1994; Allendorf and Luikart 2007). The current levels of extirpations and the 
widespread decline in the number of taxon (e.g. bear (Ursus arctos), bighorn sheep (Ovis canadensis)), are prompting conservation mangers to incorporate reintroductions into species recovery plans (e.g. giant tortoises (Geochelone nigra hoodensis) and cactus (Opuntia megasperma var. megasperma) Gibbs et al. 2008; tuatara (Sphenadon guntheri) Nelson et al. 2002; Przewalski horse (Equus ferus przewalskii) Van Dierendonck and Wallis de Vries 1996). If the remaining number of individuals in a population of an endangered species is extremely small, genetic considerations may be overlooked for pragmatic reasons (e.g. necessity to increase the number of individuals). However, if populations are in recovery, genetic diversity should not be neglected. If a population is small enough to be conservation-reliant, it is likely to be prone to loss of genetic diversity and inbreeding, which may affect evolutionary potential (Frankham et al. 1999; Frankham 2005), meaning that a loss in the level of genetic variation might limit a population's suitability or adaptability to its environment (Soulé 1980; Allendorf and Leary 1986b). Management of black rhino has mainly focused on protecting existing populations, creating new populations through the means of reintroductions, translocations and supplementations, and through captive breeding programmes (Emslie et al. 2007).

Black rhino genetic studies have examined levels of genetic variation and differentiation among and between the subspecies (Ashley et al. 1990; Swart et al. 1994; Harley et al. 2005), but the definition of "acceptable levels" of genetic variation differ between studies. As researchers, we need to provide quantitative evidence to wildlife managers in a way that helps them in the field to secure the evolutionary potential of the black rhinoceros (e.g. identifying individuals that are ideal translocation candidates). Despite work in the field of 
black rhino genetics over the past two decades, field mangers only have a general idea of how their subspecific populations differ genetically from other populations.

\subsubsection{Black Rhinoceros Genetics}

As the black rhino species recovery progresses, it is now necessary for metapopulation management to shift in emphasis from size and growth to population quality indicators, such as levels of genetic variation. Despite an increase in the total number of black rhino, sample sizes for genetic studies tend to have been small due to the difficulty in collecting genetic material. Small sample sizes typically underestimate diversity measures like allelic richness (also called allelic diversity) and heterozygosity. Nei (1978) demonstrated that when estimating average heterozygosity (measure of genetic variation within a population), a small number of individuals could be used if a large number of loci (more than 50) were used in the study and the average heterozygosity is low. Nei (1978) also established that a small number of individuals could be sampled for determining genetic distance if the gentic distance was large and the average heterozyogisty of the two species being compared was low.

The following are studies that have included black rhino. Most of the studies vary in sample size, examine fewer than 50 loci, and the genetic distance between the subspecies is not large. The combination of these limitations has occassionally lead to contradictory results. While each of these studies has contributed to the understanding of rhino genetics, there are still information gaps. 


\section{Allozymes}

Allozymes are soluble protein-coding enzymes, usually taken from blood, kidney or liver, mixed with a buffer and separated by their charge or molecular weight on an eletrophoresis gel. Allozyme markers are used to evaluate genetic variation, population structure and gene flow (Lowe et al. 2004). They are inexpensive, easy to detect and generally selectively neutral (Lowe et al. 2004); however they are limited in that they do not directly measure the amount of DNA variation within a population (Conner and Hartl 2004).

Merenlender et al. (1989) observed a significant lack of genetic variability across 25-30 loci in the four rhino taxa (C. s. simum, C. s. cottoni, D. bicornis and $R$. unicornis) they examined and more specifically, low amounts of genetic variation within D. b. michaeli black rhino samples $(0.013$ observed heterozygosity) from Kenya and east Africa (Table 1.2), concluding that the low levels of genetic variation was likely caused by recent historic demographic bottlenecks. While the authors suggested that the results were probably not important for short-term conservation goals, they recognized that their sample sizes were small ( $<10$ samples for each taxa), which may have biased results.

Swart et al. (1994) narrowed their focus to four southern African black rhino populations (D. b. minor, Zambezi Valley, Zimbabwe (n=90); D. b. bicornis, Etosha, Namibia (n=6); D. b. minor, $\mathrm{HiP}(\mathrm{n}=25)$ and D. b. minor, Mkuze (n=34), South Africa) (Table 1.2). All populations were in Hardy-Weinberg Equilibrium (HWE), six out of 30 loci were polymorphic and one locus was sex linked $(G p-5)$. When the 24 monomorphic loci were excluded from calculations, the proportion of heterozygote individuals for the four populations was between $0.036-0.059$ with expected heterozygosity between $0.003-0.02$. The Zimbabwe samples had 
Chapter I: Introduction

Table 1.2: Genetic studies that include black rhino; Type of study, Allozyme using plasma and red blood cells (RBC), Restriction Fragment Length Polymorphism (RFLP) using mitochondrial DNA (mtDNA) and microsatellites (msats) for black rhino (BR) and white rhino (WR); Number of restriction sites and number of loci used in the study, Sample Size, Species/Subspecies, Sample Country of Origin, Expected heterozygosity $\left(H_{e}\right)$, Observed heterozygosity $\left(H_{0}\right)$, Number of mtDNA haplotypes

\begin{tabular}{|c|c|c|c|c|c|c|c|c|}
\hline Author & $\begin{array}{l}\text { Type of } \\
\text { Study }\end{array}$ & Number of sites / loci & $\begin{array}{l}\text { Sample } \\
\text { Size }\end{array}$ & $\begin{array}{l}\text { Species/ } \\
\text { Subspecies }\end{array}$ & Sample Origin & $\sim H_{e}$ & $\sim H_{0}$ & $\begin{array}{l}\text { No. of mtDNA } \\
\text { Haplotypes }\end{array}$ \\
\hline $\begin{array}{l}\text { Merenlender et al. } \\
1989\end{array}$ & $\begin{array}{l}\text { Allozyme } \\
\text { (plasma) }\end{array}$ & $25-31$ & $\begin{array}{l}9 \\
23 \\
7 \\
3\end{array}$ & $\begin{array}{l}\text { D. b. michaeli } \\
\text { C. s. simum } \\
\text { C. s. cottoni } \\
\text { R. unicornis }\end{array}$ & $\begin{array}{l}\text { Kenya/East Africa } \\
\text { South Africa } \\
\text { Sudan } \\
\text { India }\end{array}$ & $\begin{array}{l}0.013 \\
0.013 \\
0.019 \\
0.000\end{array}$ & $\begin{array}{l}- \\
- \\
-\end{array}$ & $\begin{array}{l}- \\
- \\
-\end{array}$ \\
\hline Swart et al. 1994 & $\begin{array}{l}\text { Allozyme } \\
\text { (plasma } \\
\text { \& RBC) }\end{array}$ & $\begin{array}{l}30 \\
\text { (6 polymorphic) }\end{array}$ & $\begin{array}{l}6 \\
105 \\
34 \\
25\end{array}$ & $\begin{array}{l}\text { D. b. bicornis } \\
\text { D. b. minor } \\
\text { D. b. minor } \\
\text { D. b. minor }\end{array}$ & $\begin{array}{l}\text { Namibia } \\
\text { Zimbabwe } \\
\text { South Africa (HiP) } \\
\text { South Africa (Mkuze) }\end{array}$ & $\begin{array}{l}0.013 \\
0.020 \\
0.003 \\
0.012\end{array}$ & $\begin{array}{l}0.053 \\
0.059 \\
0.038 \\
0.036\end{array}$ & $\begin{array}{l}- \\
- \\
-\end{array}$ \\
\hline $\begin{array}{l}\text { Swart \& Ferguson } \\
1997\end{array}$ & $\begin{array}{l}\text { Alloyzyme } \\
\text { (plasma } \\
\& \text { RBC) }\end{array}$ & $\begin{array}{l}30 \\
\text { (6 polymorphic) }\end{array}$ & $\begin{array}{l}21 \\
135 \\
25 \\
34\end{array}$ & $\begin{array}{l}\text { D. b. bicornis } \\
\text { D. b. minor } \\
\text { D. b. minor } \\
\text { D. b. minor }\end{array}$ & $\begin{array}{l}\text { Namibia } \\
\text { Zimbabwe } \\
\text { South Africa (HiP) } \\
\text { South Africa (Mkuze) }\end{array}$ & $\begin{array}{l}- \\
- \\
-\end{array}$ & $\begin{array}{l}0.053 \\
0.062 \\
0.038 \\
0.037\end{array}$ & $\begin{array}{l}- \\
- \\
-\end{array}$ \\
\hline Ashley et al. 1990 & $\begin{array}{l}\text { RFLP } \\
\text { (mtDNA) }\end{array}$ & $\begin{array}{l}18 \\
\text { (4 polymorphic) }\end{array}$ & $\begin{array}{l}11 \\
11 \\
1\end{array}$ & $\begin{array}{l}\text { D. b. michaeli } \\
\text { D. b. minor } \\
\text { D. b. minor }\end{array}$ & $\begin{array}{l}\text { Kenya } \\
\text { Zimbabwe } \\
\text { South Africa }\end{array}$ & $\begin{array}{l}- \\
-\end{array}$ & - & $\begin{array}{l}\left.1 \text { (type } B_{1}\right) \\
2 \text { (type } A \& B_{2} \text { ) } \\
1 \text { (type } A \text { ) }\end{array}$ \\
\hline O'Ryan et al. 1993 & $\begin{array}{l}\text { RFLP } \\
\text { (mtDNA) }\end{array}$ & $\begin{array}{l}18 \\
\text { (monomorphic } \\
\text { for D. b. minor) }\end{array}$ & $\begin{array}{l}4 \\
22 \\
2\end{array}$ & $\begin{array}{l}\text { C. s. simum } \\
\text { D. b. minor } \\
\text { D. b. minor }\end{array}$ & $\begin{array}{l}\text { South Africa } \\
\text { South Africa (HiP \& Mkuzi) } \\
\text { Zimbabwe }\end{array}$ & & & $\begin{array}{l}2 \\
1 \\
1\end{array}$ \\
\hline O'Ryan et al. 1994 & $\begin{array}{l}\text { RFLP } \\
\text { (mtDNA) }\end{array}$ & $\begin{array}{l}16 \\
\text { ( } 3 \text { polymorphic) }\end{array}$ & $\begin{array}{l}5 \\
1 \\
26 \\
1\end{array}$ & $\begin{array}{l}\text { D. b. bicornis } \\
\text { D. b. michaeli } \\
\text { D. b. minor } \\
\text { D. b. chobiensis }\end{array}$ & $\begin{array}{l}\text { Namibia } \\
\text { South Africa } \\
\text { South Africa } \\
\text { Namibia }\end{array}$ & $\begin{array}{l}- \\
- \\
-\end{array}$ & $\begin{array}{l}- \\
- \\
-\end{array}$ & $\begin{array}{l}\left.1 \text { (type } B_{1}\right) \\
\left.1 \text { (type } B_{2}\right) \\
1 \text { (type } A \text { ) } \\
1 \text { (type } A)\end{array}$ \\
\hline $\begin{array}{l}\text { Brown \& Houlden } \\
2000\end{array}$ & $\begin{array}{l}\text { Sequencing } \\
\text { (mtDNA) }\end{array}$ & $\begin{array}{l}2 \text { (forward and reverse) } \\
\text { mt15996L (forward) } \\
\text { mt1652H (reverse) }\end{array}$ & $\begin{array}{l}2 \\
9\end{array}$ & $\begin{array}{l}\text { D. b. michaeli } \\
\text { D. b. minor }\end{array}$ & $\begin{array}{l}\text { Captive Born } \\
\text { Zimbabwe \& Captive Born }\end{array}$ & - & - & $\begin{array}{l}2 \\
5\end{array}$ \\
\hline $\begin{array}{l}\text { Brown \& Houlden } \\
1999\end{array}$ & Msat & $\begin{array}{l}11 \\
\text { DB1, DB4, DB5, DB14, } \\
\text { DB23, DB30, DB42,DB44, } \\
\text { DB49, DB52,DB66 }\end{array}$ & $\begin{array}{l}7 \\
2 \\
5\end{array}$ & $\begin{array}{l}\text { D. b. bicornis } \\
\text { D. b. michaeli } \\
\text { D. b. minor }\end{array}$ & $\begin{array}{l}\text { Unknown } \\
\text { Captive Born } \\
\text { Zimbabwe \& Captive Born }\end{array}$ & $\begin{array}{l}0.686 \\
0.682 \\
0.594\end{array}$ & - & $\begin{array}{l}- \\
-\end{array}$ \\
\hline $\begin{array}{l}\text { Cunningham et al. } \\
1999\end{array}$ & Msat & $\begin{array}{l}5 \\
\text { BR3, BR4, BR6, BR17, } \\
\text { BR20 }\end{array}$ & 72 & D. bicornis & Unknown & 0.580 & - & - \\
\hline Garnier et al. 2001 & Msat & $\begin{array}{l}10 \\
\text { BR4, BR6, BR17 } \\
\text { (Cunningham et al. 1999) } \\
\text { DB1, DB5, DB23, DB44, } \\
\text { DB49, DB52, DB66 } \\
\text { (Brown \& Houlden 1999) }\end{array}$ & 35 & D. b. minor & Zimbabwe & 0.616 & 0.726 & - \\
\hline Harley et al. 2005 & Msat & $\begin{array}{l}9 \\
\text { BR4, BR6, BR17 } \\
\text { (Cunningham et al. 1999) } \\
\text { DB1, DB14, DB44, DB49, } \\
\text { DB52, DB66 } \\
\text { (Brown \& Houlden 1999) }\end{array}$ & $\begin{array}{l}19 \\
47 \\
1 \\
1 \\
53\end{array}$ & $\begin{array}{l}\text { D. b. michaeli } \\
\text { D. b. minor } \\
\text { D. b. chobiensis } \\
\text { D. b. longipes } \\
\text { D. b. bicornis }\end{array}$ & $\begin{array}{l}\text { Kenya } \\
\text { South Africa \& Zimbabwe } \\
\text { southern Angolia } \\
\text { Camaroon } \\
\text { Namibia }\end{array}$ & $\begin{array}{l}0.675 \\
0.459 \\
- \\
- \\
0.505\end{array}$ & $\begin{array}{l}0.731 \\
0.436 \\
- \\
- \\
0.523\end{array}$ & $\begin{array}{l}- \\
- \\
- \\
-\end{array}$ \\
\hline Nielsen et al. 2008 & Msat & $\begin{array}{l}5 \text { (WR) } 12 \text { (BR) } \\
\text { WR: AY138542, AY138543, } \\
\text { AY138544, AY138545, } \\
\text { AY138541 } \\
\text { BR: AF12972, AF129726, } \\
\text { AF129727, AF129729, } \\
\text { AF129730, AF129734, } \\
\text { AF129732, AY606078, } \\
\text { AY606079, AY606080, } \\
\text { AY606082, AY606083 } \\
\text { (Nielsen et al. 2008) }\end{array}$ & $\begin{array}{l}22 \\
6\end{array}$ & $\begin{array}{l}\text { C. s. simum } \\
\text { D. b. minor }\end{array}$ & $\begin{array}{l}\text { South Africa (HiP) } \\
\text { South Africa (HiP) }\end{array}$ & $\begin{array}{l}0.420 \\
0.372\end{array}$ & $\begin{array}{l}0.436 \\
0.322\end{array}$ & - \\
\hline Karsten et al. 2011 & Msat & $\begin{array}{l}10 \\
\text { BR4, BR6, BR17 } \\
\text { (Cunningham et al. 1999) } \\
\text { DB1, DB14, DB49, DB66 } \\
\text { (Brown \& Houlden 1999) } \\
\text { AY606078, AY606080, } \\
\text { AY606083 } \\
\text { (Nielsen et al. 2008) }\end{array}$ & $\begin{array}{l}77 \\
4 \\
4\end{array}$ & $\begin{array}{l}\text { D. b. minor } \\
\text { D. b. bicornis } \\
\text { D. b. michaeli }\end{array}$ & $\begin{array}{l}\text { South Africa \& Zimbabwe } \\
\text { Namibia } \\
\text { South Africa \& Tanzania }\end{array}$ & $\begin{array}{l}0.44 \\
0.43 \\
0.54\end{array}$ & $\begin{array}{l}0.38 \\
0.46 \\
0.54\end{array}$ & - \\
\hline Muya et al. 2011 & Msat & $\begin{array}{l}\text { BR4, BR6, BR17 } \\
\text { (Cunningham et al. 1999) } \\
\text { DB1, DB5, DB30, DB44, } \\
\text { DB52, DB66 } \\
\text { (Brown \& Houlden 1999) }\end{array}$ & 145 & D. b. michaeli & Kenya & 0.69 & 0.70 & - \\
\hline $\begin{array}{l}\text { Van Coeverden de } \\
\text { Groot et al. } 2011\end{array}$ & Msat & $\begin{array}{l}\text { DB1, DB44, DB52, DB66 } \\
\text { (Brown \& Houlden 1999) } \\
\text { BR4, BR6, BR17 } \\
\text { (Cunningham et al. 1999) } \\
\text { BIRh2B, Blrh37D } \\
\text { (Van Coeverden de Groot } \\
\text { et al. 2011) }\end{array}$ & 144 & D. b. bicornis & Namibia & 0.51 & 0.52 & - \\
\hline
\end{tabular}


the highest gene diversity, while Mkuze had the lowest. The authors suggested that the two KZN populations (HiP and Mkuze) were not genetically depauperate and many southern African black rhino populations had similar genetic variation to populations of "some outbreeding mammal species", although the authors do not site examples of said outbred species. Swart et al. (1994) also propose that the level of genetic variation in the southern African populations was representative of the heterozygosity present before drastic population declines and that this would be advantageous to captive breeding programmes.

Swart and Ferguson (1997) studied two subspecies of black rhino (one $D$. b. bicornis and three D. b. minor populations) (Table 1.2). They concluded that the four populations were conspecific isolated remnants of a large ancestral population; none of the populations belonged to discrete subspecies but were instead part of a west-to-east 'genetic continuum' where by the Etosha (Namibia) and KZN (South Africa) populations are the extremes, but mere subsets of the Zambezi (Zimbabwe) population. They concluded that short-term genetic management for the species was unnecessary due to large genetic variation and no evidence of inbreeding or excess in homozygosity. However, they concluded that the levels of variation in the Zimbabwe D. b. minor population indicate that it is the only population of black rhino to retain prebottleneck levels of genetic variation. They recommended immediate genetic management in order to maintain the level of variability in the Zambezi, Zimbabwe population. 


\section{Mitochondrial DNA (mtDNA)}

MtDNA consists of a haploid circular molecule found in the cellular mitochondria of most eukaryotes. It is typically maternally inherited in mammals and lacks recombination due to the nature of its replication process. MtDNA is more sensitive to changes in population demography because it has a quarter the effective population size $\left(\mathrm{N}_{\mathrm{e}}\right)$ compared with nuclear loci. MtDNA has a relatively high mutation rate and shows higher levels of polymorphism compared to many nuclear genes making it useful when looking for patterns of genetic differentiation (Moritz et al. 1987). Studies of mtDNA can be used effectively in long-term and short-term management of populations, more specifically to (1) measure genetic variation in recently declining populations (2) define Evolutionarily Significant Units (ESUs) and (3) to ascertain evolutionary or phylogenetic conservation value of populations (Moritz 1994). Studies on black rhino mtDNA include the use of restriction maps and direct DNA sequencing of the control region and 12S rRNA which have indicated differences between the black rhino subspecies and suggest there may be population differentiation (Harley et al. 2005).

\section{Restriction Enzymes Analysis of mtDNA}

Restriction fragment length polymorphisms (RFLP) are used to evaluate genetic variation, population structure and gene flow; however, restriction enzymes identify differences in the sequence of DNA, not the expressed proteins (Lowe et al. 2004). RFLP results are repeatable and a considerable amount of variation can be identified if the right combination of restriction enzymes is developed. 
Unfortunately, this method can be expensive, time consuming and combining results from different labs can be difficult (Lowe et al. 2004).

Ashley et al. (1990) examined mtDNA RFLPs of black rhinos from three different geographic populations: Zimbabwe (D. b. minor, $\mathrm{n}=11)$, South Africa $(D$. b. minor, $\mathrm{n}=1$ ) and Kenya (D. b. michaeli, $\mathrm{n}=11$ ) (Table 1.2). They found a small amount of intraspecific variation, with only three mtDNA haplotypes; one unique haploptype in Kenya (D. b. michaeli), one unique haplotype in Zimbabwe (D. b. minor) and one shared with Zimbabwe and South Africa (D. b. minor). The DNA sequences of all three haplotypes were similar which led the authors to conclude that the subspecies had recently shared a common ancestor.

O'Ryan and Harley (1993) used mtDNA restriction maps using 18 restriction endonucleases and estimated that the time divergence from a common ancestor for black rhino and white rhino was $3.4 \times 10^{6}$ years ago. Black rhino samples were from HiP $(n=16)$, Mkuze $(n=6)$ and Zimbabwe $(n=2)$ (Table 1.2). White rhino samples $(n=4)$ were from HiP. There were no polymorphic sites detected in the restriction map of the black rhino and only one polymorphic site in the white rhino. The results contrasted with those of Ashley et al. (1990) who also used a restriction-fragment size approach, but were consistent with Merenlender et al. (1989) allozyme findings of small intraspecific variation.

O'Ryan et al. (1994), again using a mtDNA restriction map, analyzed differentiation among black rhino subspecies and populations. Their subspecies samples included D. b. minor $(\mathrm{n}=26)$, D. b. bicornis $(\mathrm{n}=5)$, D. b. michaeli $(\mathrm{n}=1)$ and D. b. chobiensis $(\mathrm{n}=1)$ (Table 1.2). In their findings, they recommended discarding D. b. chobiensis as a subspecies and placing it in the south-central 
ecotype with $D . b$. minor. Their results showed little variation with only two site differences between 33 individuals from three different geographic regions and monomorphic mtDNA restriction maps within the same geographic region.

\section{MtDNA Sequencing}

DNA sequencing typically involves the amplification of a segment of DNA (e.g. mitochondrial DNA control region) via polymerase chain reaction (PCR) and it is a direct measure of genetic variation.

Tougard et al. (2001) sequenced the mtDNA 12S rRNA in order to establish where Sumatran rhino fit within the other four extant Asian and African species. They narrowed the divergence time of the species to approximately 26 million years ago with the Sumatran rhino forming a sister group of the genus Rhinoceros. Later, Fernando et al. (2006) used mtDNA 12S rRNA and control region sequences to examine the genetic divergence and level of variation within and between two extant Javan rhino populations based on samples from all five extant rhino species. They established that the two populations each formed a discrete ESU and recommended independent management of each population.

Brown and Houlden (2000) sequenced the non-coding mtDNA control region of captive D. b. michaeli $(\mathrm{n}=2)$ and wild captured Zimbabwe D. b. minor $(n=9)$ to examine evolutionary relationships. Five haplotypes were found in the nine $D$. $b$. minor samples with a haplotype diversity of 0.86 (Table 1.2 ). Both $D . b$. minor and $D . b$. michaeli were shown to be reciprocally monophyletic, meaning that all members of a lineage share a more recent common ancestor, with a divergence time between the two subspecies ranging from 0.92 to 1.3 MYA. 


\section{DNA Microsatellites}

DNA microsatellites are a type of co-dominant DNA marker used extensively in contemporary population and evolutionary genetic studies. (Slatkin 1995b). They are tandem repeats usually between one and five base pairs long (Jarne and Lagoda 1996) that are typically non-coding and are not influenced by the selection process (Slatkin 1995). Microsatellites are found throughout an organism's genome and are surrounded by unique DNA sequences, which enable primers to be designed and used to amplify each locus separately (Jarne and Lagoda 1996). The mutation rate of microsatellites is important because the rate of change and model of mutation help determine population structure (Jarne and Lagoda 1996). Microsatellite mutations are approximately $10^{-5}$ to $10^{-2}$ higher than seen in allozymes. Mutations occur through slippage through increases and decreases in the number of unit repeats (Jarne and Lagoda 1996). Brown and Houlden (1999) and Cunningham et al. (1999) were the first to specifically isolate microsatellite sequences from black rhinos followed by Nielsen et al. (2008) and Van Coeverden de Groot et al. (2011) (Table 1.2). Brown and Houlden (1999) designed 11 microsatellite marker primers to assess genetic diversity within an Australian ex situ breeding and conservation programme of D. b. michaeli (originally from Kenya $(\mathrm{n}=2)$ and D. b. minor (originally from Zimbabwe $(\mathrm{n}=5)$ ). The mean expected heterozygosity $\left(H_{\mathrm{E}}\right)$ over 11 loci for D. b. minor was $0.594 \pm 0.068$ and $0.682 \pm 0.085$ for D. b. michaeli.

Garnier et al. (2001) conducted a genetic analysis using ten microsatellites and used DNA from faecal samples to increase the understanding of the mating system, reproductive skew and effective population size of a D. b. minor population $(\mathrm{n}=35)$ in Save Valley, Zimbabwe 
(Table 1.2). This was the first study to provide genetic evidence of both polygyny and a male reproductive skew in black rhino. The Save Valley population was founded with individuals from Zambezi, Zimbabwe, which Swart and Ferguson (1997) suggested had the highest level of genetic variation of all black rhino populations and probably reflect that of a pre-bottleneck population. The published genotypes provide a genetic record that will enable conservation managers of other Zambezi founder populations to compare the levels of genetic variations within their own populations. These data will also be helpful in the future to the Save Valley population as a decrease in the level of variability will be easily identifiable.

Harley et al. (2005) used nine of the black rhino microsatellite markers to establish baseline information regarding levels of genetic diversity and population differentiation in black rhino subspecies (D. b. bicornis $(\mathrm{n}=53) ; D . b$. minor $(\mathrm{n}=47) ;$ D. $b$. michaeli $(\mathrm{n}=19)$ and the now extinct $D$. b. longipes $(\mathrm{n}=1)$ and D. b. chobiensis $(\mathrm{n}=1)$ ) (Table 1.2). They found that D. b. michaeli had the highest level of genetic diversity with an expected heterozygosity $\left(H_{\mathrm{E}}\right)$ of 0.675 followed by D. b. bicornis $\left(H_{\mathrm{E}}=0.505\right)$ and D. b. minor $\left(H_{\mathrm{E}}=0.459\right)$. The authors point out that the D. b. minor results may indicate a level of population substructure due to samples coming from a number of games reserves in South Africa and Zimbabwe. The possible population substructure of D. b. minor was not important to their study however, and was therefore, not investigated.

Nielsen et al. (2008) designed 21 microsatellites for both black and white rhino, seven of which were polymorphic and were used to distinguish the two species from each other (Table 1.2). The authors do not specify with which subspecies of black rhino $(n=6)$ they were working, but an assumption can be 
made that the subspecies is $D . b$. minor as the authors stated that the samples were from HiP.

When comparing results among genetic studies with differing sample sizes, expected heterozygosity is preferred, as it takes into account sample size variation. Nielsen et al. (2008) reported their microsatellite observed heterozygosity $\left(H_{0}=0.322\right)$, instead of $H_{\mathrm{E}}(0.372)$ for two out of three of the comparisons with other black rhino studies and also compared their results against different black rhino subspecies. Since the aim of their study was to differentiate black rhino samples from white rhino samples, identifying differences between black rhino subspecies was not considered important. That being said, they concluded that the wild black rhino population in South Africa retains a moderate degree of allele diversity. They reached this conclusion by stating that their black rhino $H_{0}=0.322(\mathrm{n}=6)$ was lower than Brown and Houlden's (1999) $H_{0}=0.660$, however the $H_{0}$ the authors reported for Brown and Houlden (1999) was the mean $H_{0}$ of two black rhino subspecies (D. b. minor, $H_{0}=0.594(\mathrm{n}=5)$ and D. b. michaeli, $\left.H_{O}=0.682(\mathrm{n}=2)\right)$. Brown and Houlden (1999) used 11 microsatellites, six of which were used by Nielsen et al. (2011). When comparing their microsatellite results to Garnier et al.'s (2001) D. $b$. minor $(\mathrm{n}=35)$, the authors used Garnier et al.'s (2001) $H_{0}=0.726$ instead of $H_{\mathrm{E}}$ $=0.62$. For their last result comparison, Nielsen et al. (2008) compared their $H_{\mathrm{E}}$ $=0.372$ with that of Harley et al. (2005), except they only reported the results for Harley et al.'s (2005) D. b. michaeli, $H_{\mathrm{E}}=0.68(\mathrm{n}=19)$, which had the highest $H_{\mathrm{E}}$ of each of the three subspecies in Harley et al's (2005) study (D. b. minor, $H_{\mathrm{E}}$ $=0.46(\mathrm{n}=46) ; D$. b. bicornis, $\left.H_{\mathrm{E}}=0.51(\mathrm{n}=53)\right)$ (Table 1.2). Finally, due to the nature of their study, the $H_{\mathrm{E}}$ and $H_{\mathrm{O}}$ results Neilsen et al. (2008) reported for the 
black rhino in their study included the use of both black rhino and white rhino loci instead of separating out the data between the two species. When specific white rhino marker results are excluded, the black rhino $H_{\mathrm{E}}$ and $H_{\mathrm{O}}$ increase to 0.439 and 0.411 respectively, much higher that the reported mean $H_{\mathrm{E}}$ and $H_{\mathrm{O}}$ of 0.372 and 0.322 , respectively.

Muya et al. (2011) focused on 12 of 16 extant D. b. michaeli populations in Kenya using both mtDNA control region sequencing and nine microsatellite loci (Table 1.2). They confirmed previous studies (Harley et al. 2005) of moderate to high levels of genetic diversity in their D. b. michaeli metapopulation, reporting a mean mtDNA haplotype diversity $(h)$ of $0.73 \pm 0.14$ with mean microsatellite $H_{\mathrm{E}}$ and $H_{\mathrm{O}}$ of $0.70 \pm 0.087$ and $0.69 \pm 0.034$ respectively.

Van Coeverden de Groot et al. (2011) used nine polymorphic microsatellite loci to examine genetic diversity and structure of $D$. b. bicornis $(n=144)$ individuals of Etosha National Park, Namibia (Table 1.2); a population that experienced a significant population increase due to increased protection. The results were to be utilized as a baseline with which conservation managers can measure changes in the level of genetic variation in the future. Mean expected heterozygosity for the samples was 0.51 , similar to levels published for D. b. bicornis by Harley et al. (2005).

Karsten et al. (2011) used 10 microsatellites to evaluate levels of genetic diversity, differentiation and inbreeding among D. b. minor $(\mathrm{n}=74)$ in seven game reserves in KZN, South Africa and a single population of D. b. minor $(\mathrm{n}=3)$ in Zimbabwe that was founded (and is managed separately from native Zimbabwe D. b. minor) with black rhino from KZN. They also compared the 
results to $D . b$. bicornis $(\mathrm{n}=4)$ and $D . b$. michaeli $(\mathrm{n}=4)$ (Table 1.2$)$. They found low levels of differentiation among KZN metapopulation and their microsatellite variation over 10 loci in D. b. minor $\left(H_{\mathrm{E}}=0.44\right)$ was lower than for the other two subspecies $\left(H_{\mathrm{E}}=0.54\right.$ for D. b. michaeli and $H_{\mathrm{E}}=0.43$ for D. b. bicornis $)$. The authors stated that the KZN D. b. minor values still fell within the range of other large mammals across Africa (African buffalo (Syncerus caffer) $H_{\mathrm{E}}=0.76$ and $H_{0}$ $=0.73$ (van Hooft et al. 2000) and $H_{\mathrm{E}}=0.58$ and $H_{0}=0.52$ (Simonsen et al. 1998), African elephant (Loxodonta africana africana) $H_{\mathrm{E}}=0.96$ and $H_{0}=0.37$ (Whitehouse and Harley 2001), Black wildebeest (Connochaetes gnou) $H_{\mathrm{E}}=0.35$ (Grobler et al. 2005), Blue wildebeest (C. taurinus) $H_{\mathrm{E}}=0.65$, (Grobler et al. 2005), Cape Mountain zebra (Equus zebra zebra) $H_{\mathrm{E}}=0.38$ and $H_{0}=0.24$ (Moodley and Harley 2005), Hartmann's mountain zebra (E. z. hartmannae) $H_{\mathrm{E}}$ $=0.54$ and $H_{0}=0.48$ (Moodley and Harley 2005)). While it is true that the $H_{\mathrm{E}}=$ 0.44 for $D$. b. minor falls within the $0.38-0.96$ of the other large mammals they listed, caution should be exercised when comparing levels of genetic variation across species since experiments that do not include the entire genome (e.g. microsatellite) represent a small portion of total DNA (Selander and Johnson 1973). The authors concluded that the use of translocations within the KZN metapopulation has helped D. b. minor retain acceptable amounts of genetic diversity and further concluded there was no need to change how black rhino in KZN were managed. They, however, missed an opportunity to compare regional levels of microsatellite variation for $D . b$. minor of KNZ to native Zimbabwe $D . b$. minor by not comparing their findings to the genotype data provided in Garnier et al.'s (2001) paper. 
The levels of genetic variation within and between populations of $D . b$. minor from different regions (e.g. South Africa and Zimbabwe) should be examined separately with comparable and repeatable methods (e.g. similar microsatellite DNA markers) to ensure accuracy. With successful reintroductions of South African black rhino continuing into areas where they have been extirpated, expansion will eventually mean that regional populations may meet and become part of the same management scheme. However, genetic considerations (e.g. differentiation) need to be addressed to ensure that longterm viability of regional populations is not compromised by outbreeding.

\subsection{Management}

\section{Translocation and Reintroductions}

A translocation is the well-planned movement of animals from one part of their range to another (IUCN 1987). It is a powerful tool used by conservation mangers to reintroduce animals to areas where they have been extirpated or have undergone dramatic declines in their distribution ranges (e.g. big horn sheep (Ovis canadensis), Singer et al. 2000; black bears (Ursus americanus) Smith and Clark 1994); to genetically augment existing populations (whitespotted charr (Salelinus leucomaenis) Yamamoto et al. 2006) and establish extralimital populations to reduce the possibility of species loss from catastrophe (e.g. Rarotonga Monarch (Pomarea dimidiata) (Griffith et al. 1989; Robertson et al. 2006)).

Reduced levels of heterozygosity and allelic diversity in founder populations is well documented (Nei et al. 1975; Leberg 1992; Keller and Waller 2002), but for pragmatic reasons reintroduced populations of threatened and 
endangered species are typically founded with a small number of individuals (Griffith et al. 1989; Kerley et al. 2003). Unfortunately, those individuals are usually sourced from populations that have themselves been through a bottleneck. Since reintroduced populations often only retain a small portion of the genetic variation from the source at functional and neutral loci, if the source population has experienced a genetic bottleneck, the rate of loss of genetic variation in the founder population may be accelerated (Bijlsma et al. 2000; Keller and Waller 2002; Leberg 1993). Establishing founder populations with individuals from different populations of the same subspecies (different genetic stock) or with high levels of genetic variation may be more likely to be successful (e.g. increased fitness)(Leberg 1993).

Reintroductions of megaherbivores like elephants and rhino require different treatment than smaller animals for various reasons. Some of these include: (1) the nature of plant abundance and disturbance due to their size, (2) they do not persist outside of conservation areas, (3) they are charismatic species and attract attention (e.g. tourism, conservation), and (4) management techniques are well-developed (Kerley et al. 2003). The primary reason for establishing founder populations of megaherbivores is because parks and reserves can no longer support (e.g. nutritionally) an increase in the number of individuals in established populations.

The first successful black rhino translocation from HiP was to Ndumo Game Reserve in 1962 (Table 1.1), coordinated under the Natal Parks Game and Fish Preservation Board (Hitchins 1984). Successful translocations from HiP continue today under current management of Ezemvelo KwaZulu-Natal Wildlife. Like HiP, Mkuze Game Reserve (MGR) is home to the only other relict 
population of D. b. minor (n= 45; D. Kelly, pers. comm.) in South Africa. However, MGR is smaller than $\operatorname{HiP}(38,000$ ha compared to $\sim 96,000$ ha) and translocations out of MGR occur less frequently than from HiP.

Receiving sites for black rhino translocations include other KZN reserves capable of supporting black rhino (e.g. Ndumo Game Park, Tembe Elephant Reserve, Ithala Gave Reserve, Zululand Rhino Reserve, Pongola Game Reserve, Phinda Resource Reserve, Weenan Game Reserve and Eastern Shores Game Reserve; Figure 1.3). As per recommened guidelines, when black rhino populations within smaller, established KZN reserves approach $75 \%$ of their estimated carrying capacities, individuals are removed and used for reintroductions elsewhere. In addition to parks within $\mathrm{KZN}$, receiving translocation sites also include parks outside KZN like Kruger National Park, Pilanesberg Game Reserve and across borders (e.g. Zimbabwe, Mozambique and Swaziland; Hitchins 1984, Emslie et al. 2009). As translocation success rates improve, it becomes increasingly necessary to study the genetic diversity of various black rhino populations in order to plan long-term management of the species (Emslie and Brooks 1999).

\section{The Need for a Paradigm Shift in Rhino Conservation Practice}

As black rhino species recovery continues, the focus of conservation managers on population growth (number of black rhino) will need to be replaced by that of population quality (e.g. genetic variation and $\mathrm{N}_{\mathrm{e}}$ ). Marked decreases in levels of genetic variation in small and recovering populations could decrease fitness or limit the long-term capacity of a population to respond to changes in the environment (Westemeier et al. 1998). The shift in focus is important because if 
genetic factors are disregarded, it could lead to inappropriate recovery strategies (Frankham 2005). If rhino poaching can be reduced and current population trends continue, in 20 years black rhino are likely to be regarded as a conservation success, much as the southern white rhino conservation is today.

While it is possible to have high levels of genetic variation represented by a few individuals forming a founder population, variation may be lost if the population remains small (Lande and Barrowclough 1987). Currently, black rhino from HiP are used to reintroduce the species back into its historic range. However, no individuals immigrate (naturally or assisted through translocations) back into HiP, meaning that the HiP population is unable to benefit from the expansion in the metapopulation, which would slow or stop a decline in levels of genetic variation. HiP has been through a population reduction and may have lost a significant amount of allelic diversity, in which case HiP could benefit from translocations either from the KZN metapopulation or possible native Zimabawe populations back into the reserve to replenish diversity. Comparing levels of genetic variation within HiP and the KZN metapopulation against native Zimbabwe populations may help with developing management schemes to prevent loss of genetic variability within the $D . b$. minor subspecies.

\section{Rhino Management Groups}

In my thesis, I will refer to several different organizations that make recommendations regarding black rhino management. Here I describe the international agencies and how they are associated with each other. There is 
considerable crossover between the groups as individuals are often affiliated with more than one organization that drafts black rhino managment guidelines.

The International Union for Conservation of Nature (IUCN)

The International Union for Conservation of Nature is a multinational organization dedicated to the conservation of nature and natural resources. The IUCN is divided into specialized groups like the Species Survival Commission (SSC), a science-based network that provides feedback to the IUCN on biodiversity, species concerns, and dispenses recommendations to specialized conservation projects. The African Rhino Specialist Group (AfRSG) is a working group within the SSC network. The group meets every two years to update rhino statistics, set priorities for populations of rhino and generate 'Action Plans'. AfRSG members are also usually involved in regional projects (Emslie et al. 2007; Emslie and Brooks 1999; Emslie et al. 2009).

\section{Regional Rhino Conservation Groups Affiliated with the IUCN}

Southern African Development Community (SADC) is an organization of southern Africa nations including Angola, Botswana, Malawi, Mozambique, Namibia, South Africa, Swaziland, Tanzania, Zambia and Zimbabwe (Emslie et al. 2009) established to promote improved standards of living in member states (SADC 2010). Food, Agriculture and Natural Resources (FANR 2010), a department within the SADC oversees the SADC Wildlife Programme of Action (WPA). The SADC WPA is responsible for managing several projects, one being the SADC Regional Programme for Rhino Conservation that focuses on conservation efforts of both black and white rhinos in southern Africa. The SADC Rhino Management Group (RMG) concentrates on black rhino efforts and 
implementing conservation plans in South Africa, Namibia, Swaziland and Zimbabwe. Members of the IUCN-AfRSG are an integral part of the SADC RMG (Emslie and Brooks 1999; Emslie et al. 2007; Emslie et al. 2009).

\section{World Wildlife Fund}

The World Wildlife Fund (WWF) South African Black Rhino Range Expansion Project (BRREP) began in 2003 and is currently directed by Dr. Jacques Flamand and coordinated with Ezemvelo KwaZulu-Natal Wildlife. The project is ongoing and focuses on successful reintroduction and translocation of black rhino from HiP and MGR source populations to areas where they have been extirpated.

\subsection{Recommended Guidelines for Black Rhino Management}

The Status Survey and Conservation Action Plan for the African Rhino (SSCAP) (Emslie and Brooks 1999) and the IUCN's Guidelines for the in situ Reintroduction and Translocation of African and Asian Rhinoceros (Emslie et al. 2009) are examples of management plans detailing necessary concerns for conservation managers including genetic monitoring. The SSCAP incorporates guidance for protection, ascertaining sex and age structure of populations, estimating population sizes, recording mortalities and estimating carrying capacities, each of which is necessary as part of a comprehensive plan. It also stresses the importance of genetic diversity maintenance. Recommendations also include founding new populations in areas that formerly supported the subspecies with cohorts consisting of at least 20 individuals. In addition, guidelines also suggest that when translocating rhino to established populations, the newly translocated individuals have as little "genetic similarity" 
as possible with the receiving population. They also advise that if possible, one rhino per generation should be introduced and accurate stud books be maintained.

The IUCN re-introduction guidelines (Emslie et al. 2009) give detailed criteria for African and Asian rhino translocations for both in situ and ex situ conservation schemes. It spells out important details needed before (i.e., harvesting in existing populations, selecting rhino for translocation, nominating new locations), during (i.e., logistics, veterinary care, holding) and after (i.e., monitoring, protection) translocations take place. Regarding genetic management, the IUCN guidelines are similar to the SSCAP in that translocated individuals should be unrelated if they are being introduced to an existing population, founder populations need to be of the same subspecies that were in that particular historical range, the number of individuals in a new population should be at least 20 , and newly founded populations should be carefully monitored.

Local agencies like Ezemvelo KZN Wildlife (EKZNW) and South Africa National Parks (SANParks) together with private and community conservancies work hard to manage black rhino populations. The local private and government management groups create management plans and follow recommended guidelines to the very best of their ability considering the financial and manpower constrants many game reserves face.

\subsection{Thesis Structure}

The specific objectives of this study were to: 
1) Determine the sequence of the mitochondria DNA (mtDNA) control region of three black rhino subspecies, estimate the level of variation within the HiP source and KZN metapopulation and compare the results to native Zimbabwe D. b. minor and the other black rhino subspecies.

2) Use ten microsatellite DNA markers to estimate the levels of heterozygosity and allelic diversity in HiP and KZN metapopulation and compare the results to previously published microsatellite data of native Zimbabwe D. b. minor as well as the other black rhino subspecies to determine whether or not HiP still has an appropriate level of genetic variation to use to establish founder populations of D. b. minor.

3) Perform a Population Viability Analysis (PVA) based on genetic data from $\mathrm{HiP}$ and vital rates to model the effects of increasing population size and supplementation to determine which management scenarios would be most effective for minimizing the loss of genetic variation.

When attempting to ascertain historic gene flow, evaluate a species demographic limits and gain understanding into a species population structure, mtDNA is an excellent metric with which to start (Rubinoff and Holland 2005). MtDNA is haploid, generally maternally inherited in mammals, lacks recombination and has a high mutation rate. Chapter Two examines sequences of the highly variable mtDNA control region of the D. b. minor source (HiP) and metapopulation of KZN and compares them against previously published native 
Zimbabwe $D$. b. minor samples, as well as, samples of $D$. $b$. bicornis from Namibia and D. b. michaeli from Kenya. Identifying the level of mtDNA variation within $\mathrm{HiP}$ is the first step in determining whether or not HiP remains an appropriate source for D. b. minor for South African founder populations. This chapter is published (Appendix A) as:

Anderson-Lederer, R.M., Linklater, W.L., and P.A. Ritchie (2012) Limited mitochondrial DNA variation within South Africa's black rhino (Diceros bicornis minor) population and implications for management. African Journal of Ecology 50(4): 404-413

I have changed the format to conform to a suggested thesis format.

Chapter Three continues the investigation of genetic structure by examining ten microsatellite DNA loci to estimate the levels of heterozygosity and allelic diversity in $\mathrm{HiP}$ and the KZN metapopulation comparing them against a small number of samples of D. b. bicornis from Namibia and D. b. michaeli from Kenya as well as previously published $D . b$. minor microsatellite data including native Zimbabwe populations. This chapter is in preparation for submission to Journal of Zoology as:

Anderson-Lederer, R.M., Linklater, W.L., and P.A. Ritchie (In prep) Low levels of microsatellite DNA variation and possible management considerations for black rhino (Diceros bicornis minor) in KwaZuluNatal, South Africa

MtDNA and microsatellite DNA results help describe past and current genetic variation as well as assisting conservationists with understanding mechanisms that are responsible for variation in allele frequencies (Conner and Hartl 2004), but they are only part of a larger management picture. Chapter Four builds on the previous chapters by incorporating microsatellite DNA and HiP vital rate information into a VORTEX population viability analysis (PVA). A 
PVA was used to model the effects of increasing population size versus supplementation to determine which management scenarios would be most effective for maintaining or minimizing the loss of genetic variation in a source population.

Chapter Five is a synopsis of work completed. My findings contribute to the knowledge base already accumulated for the KZN D. b. minor metapopulation and will assist wildlife managers improve conservation plans as D. b. minor enter recovery. Results from the data chapters are reviewed and recommendations for management of black rhino are made. Shortcomings of this project are also pointed out and suggestions for future work are addressed. 

Chapter I: Introduction 


\section{CHAPTER TWO}

Limited mitochondrial DNA variation within South Africa's black rhino (D. b. minor) population and implications for management

PUBLISHED IN: The African Journal of Ecology

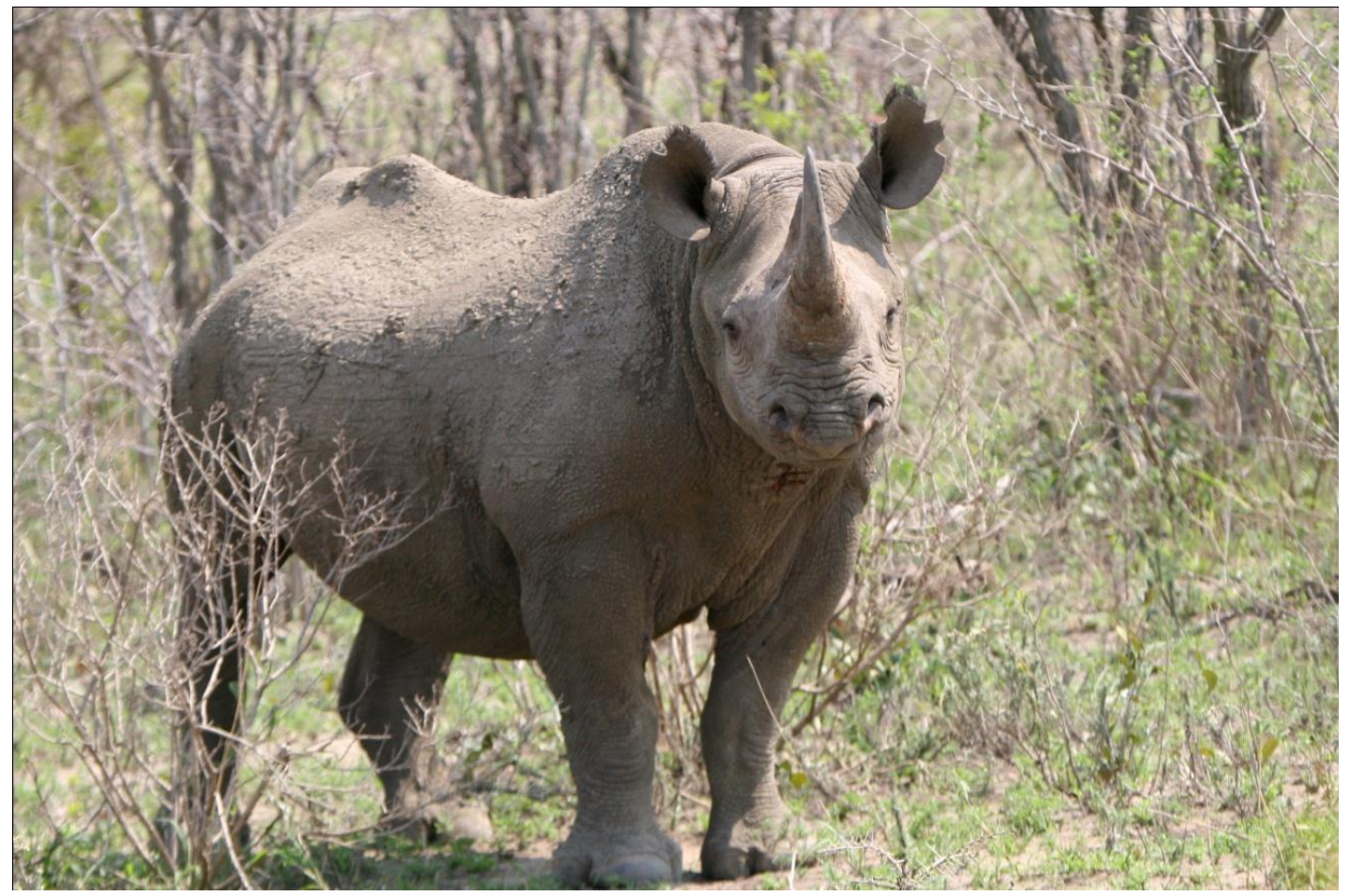

Photo by Rosalynn Anderson-Lederer 


\subsection{Abstract}

The taxonomy of African black rhinoceros (Diceros bicornis) remains unresolved. Maintaining levels of genetic diversity, and species rescue by reintroduction and restocking requires its resolution. I compared the sequences of the mitochondrial DNA (mtDNA) control region for a total of 101 D. bicornis from three subspecies: D. b. minor, D. b. michaeli and D. b. bicornis. A single unique haplotype was found within the 65 D. b. minor samples from KwaZuluNatal (KZN) Province, South Africa, 55 of which came from Hluhluwe-iMfolozi Game Park (HiP) and Mkuze Game Reserve (MGR) source populations. However, six different haplotypes were represented in 11 D. b. minor samples from Zimbabwe. Similarly, published autosomal microsatellite data indicate low levels of diversity within the KZN D. b. minor populations. The low levels of mtDNA diversity within the KZN metapopulation point to the possible need for genetic supplementation. However, there is a need to determine whether the low levels of genetic variation within KZN D. b. minor is a result of the recent bottleneck or if KZN historically always had low diversity.

\subsection{Introduction}

Species conservation depends on identifying genetically distinct groups or management units and implementing strategies to retain genetic variation. Genetically distinct populations can contain unique genetic variation and/or they can be locally adapted to their habitat. Mixing them with other populations may break up genetically complex traits and, in some cases, lead to outbreeding depression (Templeton 1986; O'Ryan et al. 1994). Alternatively, genetic differences between populations can also result from strong genetic drift caused 
by population fragmentation and declining population sizes (Frankham et al. 2002; Allendorf and Luikart 2007). When the genetic structure and historic pattern of gene flow of a species has been described, reintroduction methods can be used to secure locally adapted populations or restocking used for genetic supplementation.

Variation in mitochondrial DNA (mtDNA) is a particularly useful metric for determining population structure and history (Moritz 1994). The control region of mtDNA is highly variable and it can often be used to resolve phylogenetic relationships between closely related taxa or for describing the genetic structure within species (Moritz et al. 1987). MtDNA is maternally inherited and so does not recombine (Hayashi et al. 1985), which means it reflects a quarter the effective population size $\left(\mathrm{N}_{\mathrm{e}}\right)$ compared with nuclear loci and hence it is more sensitive to changes in population demography.

The black rhinoceros (Diceros bicornis: Perissodactyla) once ranged across the African continent and numbered in the hundreds of thousands (Western and Vigne 1985). By 1969, their numbers had declined to $\sim 65,000$ (Muya and Oguge 2000) and, during the last century, the species disappeared faster than any other large mammal (Hitchins 1975; Western and Vigne 1985). The major causes for their decline have been anthropogenic, primarily illegal hunting (Western and Vigne 1985; Emslie and Brooks 1999; Amin et al. 2006). Nevertheless, conservation efforts have seen in situ black rhino numbers increase from a low of 2,475 individuals in 1993 to approximately 4,880 in 2010 (Emslie 2011).

Three extant black rhino subspecies are recognised across Africa, including approximately 742 D. b. michaeli (Eastern black rhino), 1,922 D. b. 
bicornis (South-western black rhinoceros) and 2,216 D. b. minor (South-central black rhinoceros) (Emslie 2011). Appraisal of the black rhino subspecies was initially based on skull measurements (Zukowsky 1965; Groves 1967; du Toit 1987), however, uncertainty regarding taxonomy remained (du Toit 1987).

Although there are apparently no impervious geographic boundaries or reproductive barriers between the subspecies, they occupy different areas with distinct habitats and climates (Harley et al. 2005; Emslie and Brooks 1999). With no historical records of migration and the extent of gene flow between the subspecies unknown, some authors have speculated that each subspecies may have genetic or behavioural adaptations to their local environments (Emslie and Brooks 1999; Harley et al. 2005). Their suggestion regarding genetic differences was confirmed through recent mtDNA and autosomal DNA analyses (Merenlender et al. 1989; Ashley et al. 1990; O'Ryan and Harley 1993; O'Ryan et al. 1994; Swart and Ferguson 1997; Brown and Houlden 1999, 2000; Nielsen et al. 2008; Karsten et al. 2011; Muya et al. 2011). Thus, current black rhino management policy is for each subspecies to be managed separately in order to maintain possible local adaptive traits and minimize the risk of outbreeding depression (Templeton 1986; O'Ryan et al. 1994; Brown and Houlden 2000; Harley et al. 2005).

The largest remnant population of the critically endangered (IUCN 2008) D. b. minor subspecies is in Hluhluwe-iMfolozi Game Park (HiP) $(\mathrm{n}=\sim 220$ Clinning et al. 2009) in KwaZulu-Natal (KZN) Province, South Africa (Figure 2.1). KZN black rhino have been separated from other populations to the north (e.g. Zimbabwe) since at least the latter half of the 19th century (Swart et al. 1994). HiP and the smaller remnant in Mkuze Game Reserve (MGR) ( $n=\sim 45$ D. 


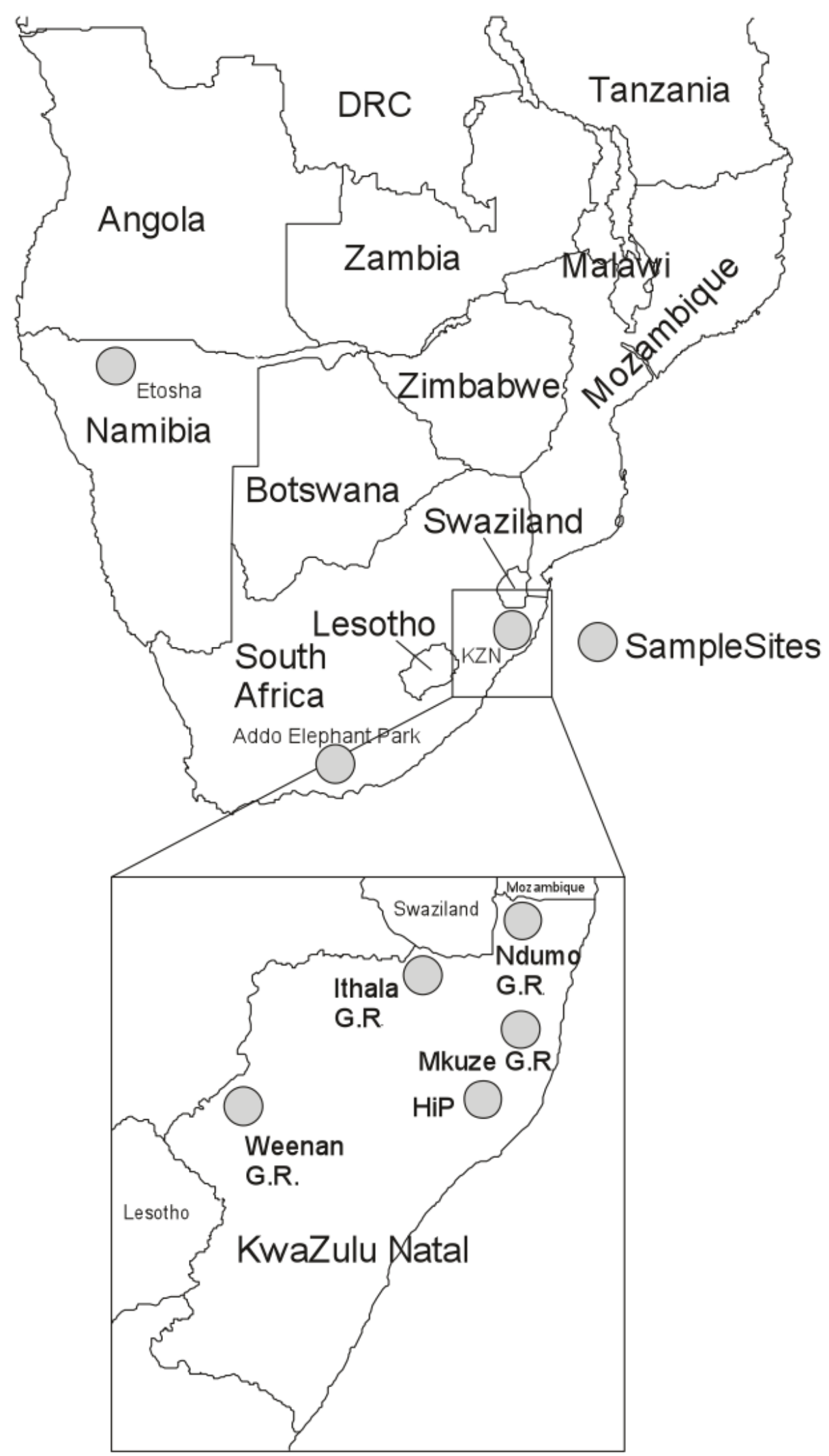

Figure 2.1: Map of Southern Africa showing black rhinoceros sample sites. Inset showing KwaZulu-Natal Game Reserves (Ndumo Game Reserve, Ithala Game Reserve, Mkuze Game Reserve, Hluhluwe-iMfolozi Game Park (HiP) and Weenan Game Reserve) 
Kelly pers. comm.) have been sources for metapopulation expansion and genetic management by reintroduction and re-stocking. Translocations from HiP to other KZN reserves first began in 1962, expanded to other South African provinces and later to other African nations (e.g., Zimbabwe, Zambia, Hitchins 1984; Emslie et al. 2009). The potential now exists for KZN D. b. minor to be mixed with D. b. minor in or from other smaller African populations, especially those in Zimbabwe, if they are not too genetically divergent. Although the KZN population will likely be strategic to the subspecies recovery throughout the African continent (Emslie and Brooks 1999), no study has yet compared the mtDNA sequences of the KZN D. b. minor metapopulation with populations outside South Africa.

The aim of this study was to use mtDNA control region sequences (406 bp) to determine the level of variation within the D. b. minor source population at $\mathrm{HiP}(\mathrm{n}=50)$ and compare it with the KZN metapopulation $(\mathrm{n}=15)$ and $D . b$. minor populations outside South Africa $(\mathrm{n}=11)$ and the other black rhino subspecies (D. b. michaeli $\mathrm{n}=21, D . b$. bicornis $\mathrm{n}=4)$. I considered the implications of the findings for the long-term management of D. $b$. minor and made recommendations for possible future research.

\subsection{Materials and Methods}

Sampling

Samples of blood and pinna ear tissue were collected from individuals of $D . b$. minor in the KZN province in South Africa (n=65), D.b. michaeli in Addo Elephant National Park, South Africa $(\mathrm{n}=1)$ and D. b. bicornis in Namibia's Northern Region (n=4) (Figure 2.1). The samples were acquired 
opportunistically during routine translocation and ear notching (for identification) events from 2002 to 2009 . Blood samples were stored in cryovials containing 1mL of DMSO/EDTA/Tris/salt solution (Seutin et al. 1991).

DNA Sequencing and Analysis

DNA extraction.

Seventy microlitres of the preserved blood solution or a $3 \mathrm{~mm} \times 3 \mathrm{~mm}$ piece of pinna ear tissue was digested in an SDS/proteinase-K solution. After dissolution, a standard phenol-chloroform DNA extraction and ethanol precipitation was conducted following the procedure of Sambrook et al. (1989).

PCR and DNA sequencing

A fragment of the mitochondrial DNA control region (406bp) was amplified using the primers mt15996L (5'-TCCACCATCAGCACCCAA-AGC-3') (Campbell et al. 1995; Brown and Houlden 2000) and mt16502H (5'- TTTGATGGCCCTGAAGTAAGAACCA- 3') (Brown and Houlden 2000; Moro et al. 1998). PCR amplifications using 1-2 $\mu \mathrm{L}$ of DNA template were carried out in $25 \mu \mathrm{L}$

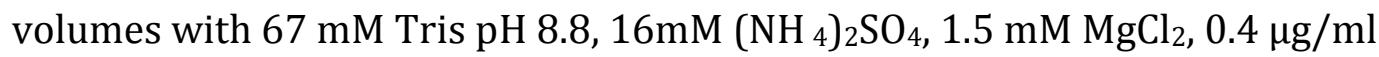
BSA, $0.4 \mu \mathrm{L}$ of each of the forward and reverse primer, $200 \mu \mathrm{M}$ of each dNTP, and 0.5 to 1 units of BIOTAQ DNA polymerase (Bioline). Thermal cycling was carried out using an Eppendorf Mastercycler for; $94^{\circ} \mathrm{C} 2 \mathrm{~min},\left(94^{\circ} \mathrm{C} 3 \mathrm{~min}, 50\right.$ $54^{\circ} \mathrm{C} 30 \mathrm{sec}, 72^{\circ} \mathrm{C} 2 \mathrm{~min}$ ), repeated for $30-40$ cycles, followed by a final step of $72^{\circ} \mathrm{C} 3 \mathrm{~min}$.

PCR products were electorphoresed in agarose gel and a molecular weight standard was used to determine the size of amplified products. Products of the correct size were purified using column purification (Roche) or ExoSAP- 
IT (GE Healthcare Lifesciences) and their DNA sequence determined using an ABI 3730 Genetic Analyzer (Massey University Genome Service).

For comparison, 11 D. b. minor sequences stored in GenBank (Accession numbers AF187825 - AF187827 \& AF187829 - AF187831, (Brown and Houlden 2000); AY742832 \& AY742833 (Fernando et al. 2006)) originally sampled from Zimbabwe and zoos in Australia and the United States were added to the data set, in addition to 20 D. b. michaeli samples (Accession numbers AF187834 \& AF187835, (Brown and Houlden 2000); AY742830 \& AY742831, (Fernando et al. 2006); FJ227484 - FJ227498, (Muya et al. 2011)) originally sampled from Kenya and zoos in Australia and the United States.

\section{Data Analysis.}

The 101 mitochondrial DNA sequences were edited by eye and then aligned using Clustal W (Larkin et al. 2007). Homogeneity of base compositions was tested using PAUP 4.0b (Swofford 2002). DnaSP v 5.10.1 (Rozas et al. 2003) was used to calculate haplotype diversity $(h)$, nucleotide diversity $(\pi)$ and standard deviation (SD) within the subspecies. The level of sequence divergence within and between populations was estimated using a pairwise distance analysis in MEGA 5.1 (Tamura et al. 2011), and standard errors were calculated using a bootstrap procedure. A statistical parsimony haplotype network was calculated with NETWORK 4.610 (Bandelt et al. 1999).

\subsection{Results}

The sequence of the mtDNA control region was determined for a total of 70 individual black rhinos as follows: D. b. minor samples: 50 from HiP, eight from Ithala, five from MGR, one from Ndumo Game Reserve, one from the 
Johannesburg Zoo (Accession number JN593089) and 11 sequences from Genbank (Accession numbers AF187826 - AF187831, AY742832 - AY742833 \& AF187832 - AF187833); D. b. michaeli samples: one from Addo Elephant Park (Accession number JN5930090) and 20 from Genbank (Accession number FJ227483 - FJ227498, AY742830 - AY742831 \& AF187834 - AF187835) and four samples for D. b. bicornis from Namibia's northern region (Accession numbers JN593091-JN593094) (Table 2.1).

The 101 aligned sequences were $363 \mathrm{bp}$ long with 31 polymorphic sites; there was an average pairwise difference of $4 \% \pm 1 \%$ between $D . b$. michaeli and D. b. minor $4.5 \% \pm 1.1 \%$ between $D$.b. michaeli and D.b. bicornis and $2.3 \% \pm$ $0.8 \%$ between $D . b$. minor and $D$. b. bicornis. No insertions or deletions were observed.

Considering each subspecies separately, the greatest level of diversity was recorded in D. b. michaeli $(\mathrm{n}=21)$, which contained 13 haplotypes and showed comparatively high nucleotide diversity $(\pi=0.011 \pm 0.00106)$ and haplotype diversity $(h=0.958 \pm 0.026)$ (Table 2.2$)$. The lowest level of diversity within subspecies was seen in the Namibian D. b. bicornis samples $(\mathrm{n}=4)$ where only one unique haplotype was found, however this was based on a small sample size and might not represent the total amount of genetic variation within the population. The pooled KZN samples and Genbank sequences of all $D$. b. minor individuals $(\mathrm{n}=79)$ contained seven haplotypes and haplotype diversity (h) was $0.267 \pm 0.067$ and a nucleotide diversity ( $\pi$ ) of $0.002 \pm 0.00063$. The eight D. b. minor Zimbabwe sequences from Brown and Houlden (2000) and two from Fernando et al. (2006) had shared haplotypes (Table 2.3), however there were no shared haplotypes with the KZN samples. 
Table 2.1: Rhinoceros subspecies and sources analyzed for mtDNA variation

\begin{tabular}{|c|c|c|c|}
\hline Subspecies & Sample Size & Sample/Sequence Source & Reference \\
\hline \multirow[t]{10}{*}{ D.b.minor } & 50 & $\begin{array}{l}\text { Hluhluwe-iMfolozi Game Park, } \\
\text { KNZ Region, Accession number } \\
\text { JN593089 }\end{array}$ & This study \\
\hline & 8 & $\begin{array}{l}\text { Itala Game Park, } \\
\text { KNZ Region }\end{array}$ & This study \\
\hline & 5 & $\begin{array}{l}\text { Mkuze Game Park, } \\
\text { KNZ Region }\end{array}$ & This study \\
\hline & 1 & $\begin{array}{l}\text { Ndumo Game Park, } \\
\text { KNZ Region }\end{array}$ & This study \\
\hline & 1 & Johannesburg Zoo & This study \\
\hline & 6 & $\begin{array}{l}\text { Chete National Park, Zimbabwe, } \\
\text { Accession number } \\
\text { AF187825 - AF187827 \& } \\
\text { AF187829 - AF187831 }\end{array}$ & Brown \& Houlden 2000 \\
\hline & 2 & $\begin{array}{l}\text { Zambezi Valley, Zimbabwe, } \\
\text { Accession numbers AY742832- } \\
\text { AY742833 }\end{array}$ & Fernando et al. 2006 \\
\hline & 1 & $\begin{array}{l}\text { Captive born, San Diego Zoo } \\
\text { Accession number AF187832 }\end{array}$ & Brown \& Houlden 2000 \\
\hline & 1 & $\begin{array}{l}\text { Captive born, Milwaulkee Zoo, } \\
\text { Accession number AF187833 }\end{array}$ & Brown \& Houlden 2000 \\
\hline & 1 & $\begin{array}{l}\text { Captive born, Western Plains Zoo } \\
\text { Accession number AF187828 }\end{array}$ & Brown \& Houlden 2000 \\
\hline \multirow[t]{5}{*}{ D.b.michaeli } & 16 & $\begin{array}{l}\text { Kenya, } \\
\text { Accession numbers } \\
\text { FJ227483-FJ227498 }\end{array}$ & Muya 2008 \\
\hline & 2 & $\begin{array}{l}\text { Solio Game Reserve, Kenya, } \\
\text { Accession numbers } \\
\text { AY742830-AY742831 }\end{array}$ & Fernando et al. 2006 \\
\hline & 1 & $\begin{array}{l}\text { Cincinnati Zoo, } \\
\text { Accession number AF187834 }\end{array}$ & Brown \& Houlden 2000 \\
\hline & 1 & $\begin{array}{l}\text { Taronga Zoo, } \\
\text { Accession number AF187835 }\end{array}$ & Brown \& Houlden 2000 \\
\hline & 1 & $\begin{array}{l}\text { Addo Elephant Park, South Africa } \\
\text { Accession number JN593090 }\end{array}$ & This study \\
\hline D.b.bicornis & 4 & $\begin{array}{l}\text { Etoshia, Namibia } \\
\text { Accession numbers } \\
\text { JN593091-JN593094 }\end{array}$ & This study \\
\hline C.c.simum & 4 & $\begin{array}{l}\text { HiP \& London Zoo } \\
\text { Accession numbers } \\
\text { AF187836-AF187839 }\end{array}$ & Brown \& Houlden 2000 \\
\hline
\end{tabular}


Chapter II: Mitochondrial DNA

Table 2.2: Mitochondrial DNA D-loop sequence variability within subspecies. Sample size $(n)$, Number of haplotypes $(H)$, haplotype diversity $(h)$, Nucleotide diversity $(\Pi)$, Standard deviation (SD)

\begin{tabular}{|c|c|c|c|c|c|c|}
\hline \multirow{3}{*}{ Subspecies: } & \multirow{3}{*}{$\mathrm{n}$} & \multicolumn{3}{|c|}{ Genetic Variability } & \multirow[b]{2}{*}{$\Pi$} & \multirow[b]{2}{*}{$(S D)$} \\
\hline & & $\mathrm{H}$ & $h$ & $(S D)$ & & \\
\hline & & & & & & \\
\hline D.b.minor (aggregate) & 76 & 7 & 0.267 & 0.067 & 0.0023 & 0.001 \\
\hline KZN meta- population & 65 & 1 & - & - & - & - \\
\hline Zimbabwe samples & 11 & 6 & 0.855 & 0.085 & 0.0074 & 0.00124 \\
\hline D.b.michaeli & 21 & 13 & 0.952 & 0.024 & 0.0112 & 0.00106 \\
\hline D.b.bicornis & 4 & 1 & - & - & - & - \\
\hline
\end{tabular}


Table 2.3: Summary statistics for the mitochondrial DNA control region sequence variability in each subspecies and haplotype identifiers used in Figure 2.2. Sample size $(n)$, Haplotypes, labeled A - U (H), haplotype diversity (h), Nucleotide diversity $(n)$, Standard deviation (SD), Number of segregating sites (S)

Subspecies \& Population:

D. b. minor

Pooled D. b. minor samples Hluhluwe-iMfolozi Game Park (KZN)

Accession number JN593089

Itala Game Park (KZN)

Mkuze Game Park (KZN)

Ndumo Game Park (KZN)

Johannesburg Zoo

Chete National Park,

Zimbabwe,

Accession number

AF187825 - AF187827 \&

AF1878329 - AF187831

Zambezi Valley, Zimbabwe,

Accession numbers

AY742832 - AY742833

Captive born, San Diego Zoo

Accession number AF187832

Captive born, Western Plains Zoo

Accession number AF187828

Captive born, Milwaulkee Zoo, Accession number AF187833

D. b. michaeli Pooled D. b. michaeli samples

Kenya,

Accession numbers

FJ227483-FJ227498

Solio Game Reserve, Kenya

Accession numbers

AY742830-AY742831

Cincinnati Zoo,

Accession number AF187834

Taronga Zoo,

Accession number AF187835

Addo Elephant Park,

South Africa

Accession number JN593090

D.b.bicornis

Pooled D. b. bicornis samples

Etosha, Namibia

Accession numbers

JN593091-JN593094

\section{Genetic Variability}

\begin{tabular}{cccccc}
$\mathrm{H}$ & $h$ & $(S D)$ & $\Pi$ & $(S D)$ & $S$ \\
\hline $\mathrm{A}-\mathrm{G}$ & 0.267 & 0.067 & 0.002 & 0.00063 & 6
\end{tabular}

A

$\begin{array}{llllll}A & - & - & - & - & - \\ A & - & - & - & - & - \\ A & - & - & - & - & - \\ A & - & - & - & - & -\end{array}$

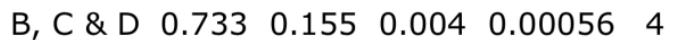

$\begin{array}{llllll}E \& G & 1 & 0.5 & 0.008 & 0.00413 & 3\end{array}$

$$
\text { E }
$$

B

$$
\text { F }
$$

$\begin{array}{llllll}H-T & 0.958 & 0.026 & 0.011 & 0.00106 & 15\end{array}$

I - S $\quad \begin{array}{lllll}0.952 & 0.031 & 0.011 & 0.00119 & 15\end{array}$

$\begin{array}{llllll}\text { J }-\mathrm{T} & 1 & 0.5 & 0.011 & 0.00413 & 3\end{array}$

$\mathrm{H}$

I

$\mathrm{T}$

U

U 
The haplotype network (Figure 2.2) shows a clear pattern of the separation among the three currently recognised subspecies with the KZN population falling out with the D. b. minor populations of Zimbabwe. Our finding of no more than three base pair substitutions between adjacent haplotypes within the D. b. michaeli subspecies is consistent with Muya et al. (2011). There is significant separation between the D. b. minor and D. b. bicornis with eight base pair substitutions as well as between D. b. minor and D. b. michaeli with nine base pair substitutions.

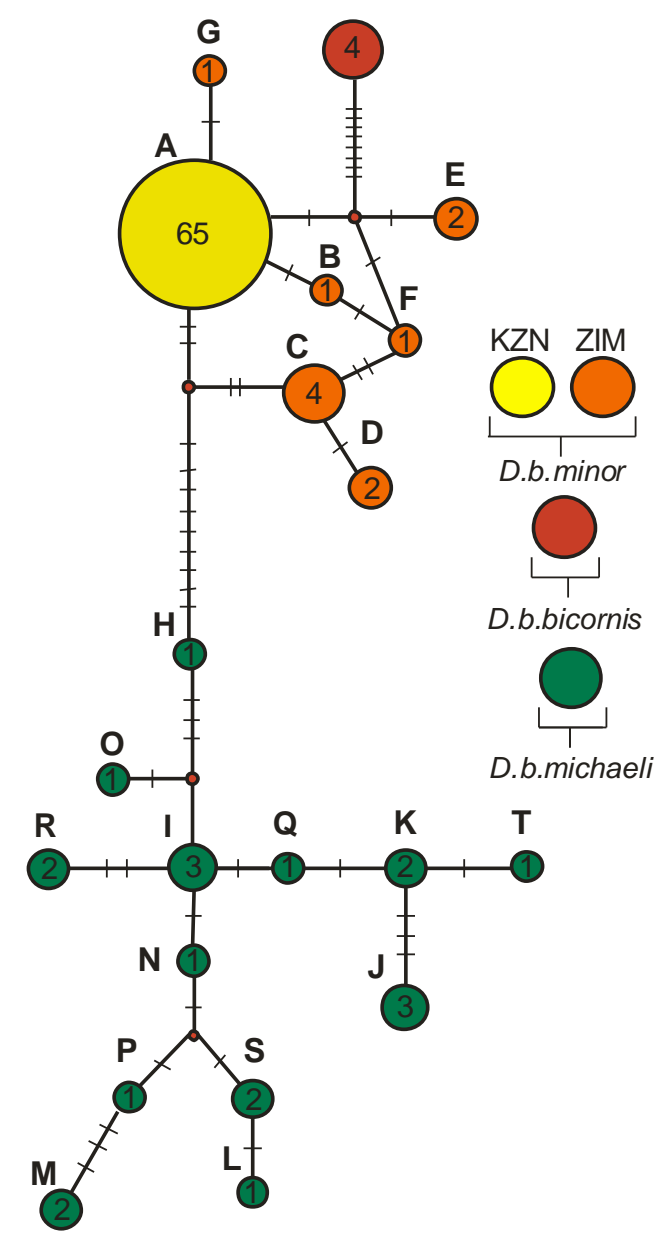

Figure 2.2: Statistical parsimony haplotype network calculated with Network Software for D. bicornis. KZN refers to the pooled D. b. minor samples within KZN (Ndumo Game Reserve, Ithala Game Reserve, Mkuze Game Reserve, Hluhluwe-iMfolozi Game Park (HiP) 


\subsection{Discussion}

I showed that the KZN population of D. $b$. minor is fixed for a single mtDNA haplotype, like most Sumatran rhino (Dicerorhinus sumatrensis) populations (Morales et al. 1997). However, unlike the Sumatran rhino populations that have occupied separate land masses for more than 10,000 years (Morales et al. 1997), it has been widely assumed that the KZN D. b. minor population has been separate from other D. b. minor populations only recently (i.e., caused by anthropogenic settlement and habitat modification during the $19^{\text {th }}$ century, Swart et al. 1994). The single mtDNA haplotype in KZN D. b. minor raises the question of whether the KZN remnant population lost genetic variation recently due to the population bottleneck, or has it been a genetically separate lineage for longer than previously thought?

MtDNA has a smaller effective population size $\left(\mathrm{N}_{\mathrm{e}}\right)$ compared to nuclear loci and is one of the first genetic markers to show the genetic signature of a demographic decline. The likelihood of two or more mtDNA haplotypes persisting within an isolated population is reduced to $\mathrm{p}<0.1$ over $4 \mathrm{~N}_{e f}$ generations and the population is expected to become monophyletic after $4 \mathrm{~N}_{e f}$ generations (Avise et al. 1984; Mucci et al. 1999). If this holds true for the KZN D. b. minor then recent population decline and fragmentation would have increased the rate of drift and might be responsible for lack of haplotype diversity within the KZN black rhinoceros. Examples of monomorphic haplotypes occurring from severe bottlenecks are well documented in several species. For instance, the Whooping Crane (Grus americana) once found throughout North America had six haplotypes in 10 pre-bottleneck museum samples, but only one haplotype persisted in the remnant post-bottleneck 
population of 14 (Glenn et al. 1999). Such rapid declines in genetic variation have also occurred amongst southern Africa's other large mammals. For example, three small remaining remnant populations of Cape mountain zebra (Equus zebra zebra) each contain a single, unique haplotype but larger Namibian populations of closely related Hartmann's mountain zebra (E. z. hartmannae) have as many as 11 different haplotypes (Moodley and Harley 2005; Watson and Chadwick 2007). Another case in point is the loss of genetic diversity at mitochondrial and Y-chromosome loci observed in small, managed populations of Cape buffalo in Kenya and Uganda which was attributed to restricted gene flow into protected areas (Van Hooft et al. 2002).

Low genetic variation is not always a consequence of recent anthropogenic fragmentation. An alternative hypothesis is that low levels of mtDNA and autosomal variation are a result of long-term demographic separation, historically small population sizes and local adaptation. For example, despite having lower mtDNA and autosomal DNA variation, there was no evidence of a genetic bottleneck in the Yellowstone National Park, U.S.A. grizzly bear (Ursus arctos) population compared to surrounding grizzly bear populations (Miller and Waits 2003). Although Yellowstone's large population is embedded within the species' range, Miller and Waits (2003) attribute the lower genetic variation to restricted gene flow into the area from the north. The common impala (Aepceros melampus melampus) of KZN also exhibited population differentiation from populations in the Limpopo Province just 490 km north. Schwab et al. (2012) attributed the genetic divergence to a narrow zone of unsuitable habitat below the eastern escarpment of the Drankensberg Mountains that impeded dispersal between the two provinces. 
Genetic replenishment by restocking and outbreeding is recommended in cases where anthropogenically induced fragmentation has caused a loss in genetic diversity and an increase in genetic divergence. For example, "genetic rescue" has been recommended for the Cape zebra (Moodley and Harley 2005; Watson and Chadwick 2007). However, where differences amongst genetically depauperate populations might be of natural origin, population management may need to take into account local adaptation and the possibility of outbreeding depression. Resolving the question regarding KZN D. b. minor mtDNA and autosomal DNA genetic structure being a recent or old event is important for guiding management plans (Rookmaaker 2005).

Microsatellite DNA markers were previously used to assess the levels of genetic variation amongst D. b. minor populations. Harley et al. (2005) found appreciable amounts of variation within the $D . b$. minor subspecies using nine microsatellite loci (Table 2.4). They recommended that as long as heterozygosity and allele numbers stayed at 'current' levels, no management policy change was necessary.

Based on a survey of 10 microsatellite DNA loci (Table 2.4), Karsten et al. (2011) found low levels of genetic variation within the KZN D. b. minor, but concluded that it was not cause for concern. They reached their conclusion based on (1) the similarity of allelic diversity and heterozygosity between the KZN D. b. minor population and the other subspecies and (2) a higher level of diversity within the black rhinoceros metapopulation compared to those found in other large African mammals. In their study, $H_{\mathrm{E}}$ estimates for the $D . b$. bicornis and D. b. michaeli subspecies (each based on only four samples) were substantially lower than those reported by Harley et al. (2005) (Table 2.4). 
Thus, estimates for D. b. bicornis and D. b. michaeli in Karsten et al. (2011) are probably underestimates. Moreover, comparisons with other large African mammals should be made cautiously. Lions in the Serengeti Plains and Ngorongoro Crater have an $H_{\mathrm{E}}$ of 0.54 and 0.46 , on par with black rhinos in Harley et al. (2005), yet unlike the Serengeti Plains lions the Ngorongoro Crater lions have a marked decrease in their reproductive rate attributed to inbreeding depression levels of genetic diversity and differentiation within and among the KZN metapopulation reported by this mtDNA study and published autosomal microsatellite data.

The likelihood of outbreeding depression in supplemented populations of the same species is low if they have the same karyotype, have been isolated for less than 500 years, and occupy similar environments (Frankham et al. 2011). Houck et al. (1995) identified variation in chromosome morphology (number of submetacentric elements) between D. b. minor and D. b. michaeli zoo samples and recommended further studies to investigate possible differences in geographically separated populations of each subspecies in the wild.

Furthermore, twenty-seven KZN D. b. minor were translocated to Malilangwe, Zimbabwe in 1997 where they were managed separately and not outbred with any Zimbabwe populations. The translocated population thrived with a growth rate of $8.3 \%$ per annum (R. du Toit pers. comm.) cf. 3.4\% over a 10-year period (1999 - 2008) in HiP (Clinning et al. 2009). The success of the translocated KZN D. b. minor in Zimbabwe alleviated concerns about the adaptability of KZN rhino to Zimbabwe. The only remaining concern is whether or not the populations have been genetically isolated for longer than previously considered. 
Table 2.4: Microsatellite results from Harley et al. 2005 and Karsten et al. 2011. Expected heterozygosity $\left(H_{\mathrm{E}}\right)$, Observed heterozygosity $\left(H_{0}\right)$

\begin{tabular}{|c|c|c|c|c|c|c|}
\hline Study & Microsatellites Used & Subspecies & Geographic region & $\begin{array}{l}\text { Sample } \\
\text { Size }\end{array}$ & $H_{\mathrm{E}}$ & $H_{0}$ \\
\hline \multirow[t]{3}{*}{ Harley et al. 2005} & BR4, BR6, BR17 & D. b. minor & South Africa and Zimbabwe & 46 & 0.46 & 0.436 \\
\hline & (Cunningham et al. 1999) & D. b. bicornis & Namibia & 53 & 0.51 & 0.523 \\
\hline & $\begin{array}{l}\text { DB1, DB14, DB44, DB49, DB52, } \\
\text { DB66 (Brown \& Houlden 1999) }\end{array}$ & D. b. michaeli & South Africa & 19 & 0.68 & 0.731 \\
\hline \multirow[t]{3}{*}{ Karsten et al. 2011} & $\mathrm{BR} 4, \mathrm{BR} 6, \mathrm{BR} 17$ & D. b. minor & South Africa & 77 & 0.44 & 0.38 \\
\hline & (Cunningham et al.1999) & D. b. bicornis & Namibia & 4 & 0.43 & 0.46 \\
\hline & $\begin{array}{l}\text { DB1, DB14, DB49, DB66 } \\
\text { (Brown \& Houlden 1999) } \\
\text { AY606078, AY606080, } \\
\text { AY606083 } \\
\text { (Nielsen et al. 2008) }\end{array}$ & D. b. michaeli & South Africa and Tanzania & 4 & 0.54 & 0.54 \\
\hline
\end{tabular}


I recommend five research tasks to assist in resolving the genetic structure of southern Africa's black rhino as a guide to future management. (1) Determine historic levels of genetic variation using museum or collection samples. (2) Investigate whether there is evidence of inbreeding depression within the $\mathrm{HiP}$ and KZN metapopulation. (3) Conduct a karyotype analysis on D. b. minor in KZN and Zimbabwe to determine whether chromosomal differences exist. (4) Increase the mtDNA sample size of the Zimbabwe D. b. minor population. Considering the high level of variation in the small sample size of the Zimbabwe sequences, a larger sample size of D. b. minor from that region might show that the KZN haplotype (A) is also there. (5) Lastly, genetic supplementation experiments should be implemented cautiously and systematically. A mixed population should be founded with at least 20 animals as suggested by du Toit (2006a), perhaps using the Malilangwe, Zimbabwe translocation event as a template or more recent guidelines (Linklater et al. 2012; Linklater et al. 2011). The translocated KZN D. b. minor rhinos in Malilangwe have not yet been outbred with the Zimbabwe rhinos (R. du Toit pers. comm.) but might be with the F1 and F2 offspring carefully monitored for signs of reduction in reproductive fitness (outbreeding depression). If the research tasks we have recommended are completed and there is evidence of historic gene flow between KZN and Zimbabwe D. b. minor and no signs of outbreeding depression in the experimentally mixed population, then KZN D. b. minor is a candidate for genetic supplementation using progeny from Zimbabwe populations. 


\subsection{Acknowledgements}

I thank Ezemvelo KZN Wildlife, specifically Drs. Dave Cooper and Dave Druce without whom this project would not have come to fruition, the Section Rangers and Game Capture Crew at Hluhluwe-iMfolozi Park, and Dr. Jacques Flamand and Pam Sheriffs of WWF's Black Rhino Range Expansion Project for their support. Thanks also to South African National Parks, specifically Michael Knight, and Ministry of Environment and Tourism, Namibia, specifically Pierre du Preez and Rudi Loutit, for facilitating earlier sampling. Thanks for financial support from the U.S. Fish and Wildlife Service administered Rhinoceros and Tiger Conservation Act of 1994 (i.e., grant agreement number 96200-9-G268), including support of other projects that facilitated earlier tissue and blood sampling (e.g. grant agreement numbers 98210-2-G363, 98210-4-G920, and 98210-6-G102). And a special thanks to Roan Plotz for assistance with sampling and data collecting. 
Chapter II: Mitochondrial DNA 


\section{CHAPTER THREE}

Low levels of microsatellite DNA variation and possible management considerations for black rhino (Diceros bicornis minor) in KwaZulu-Natal, South Africa

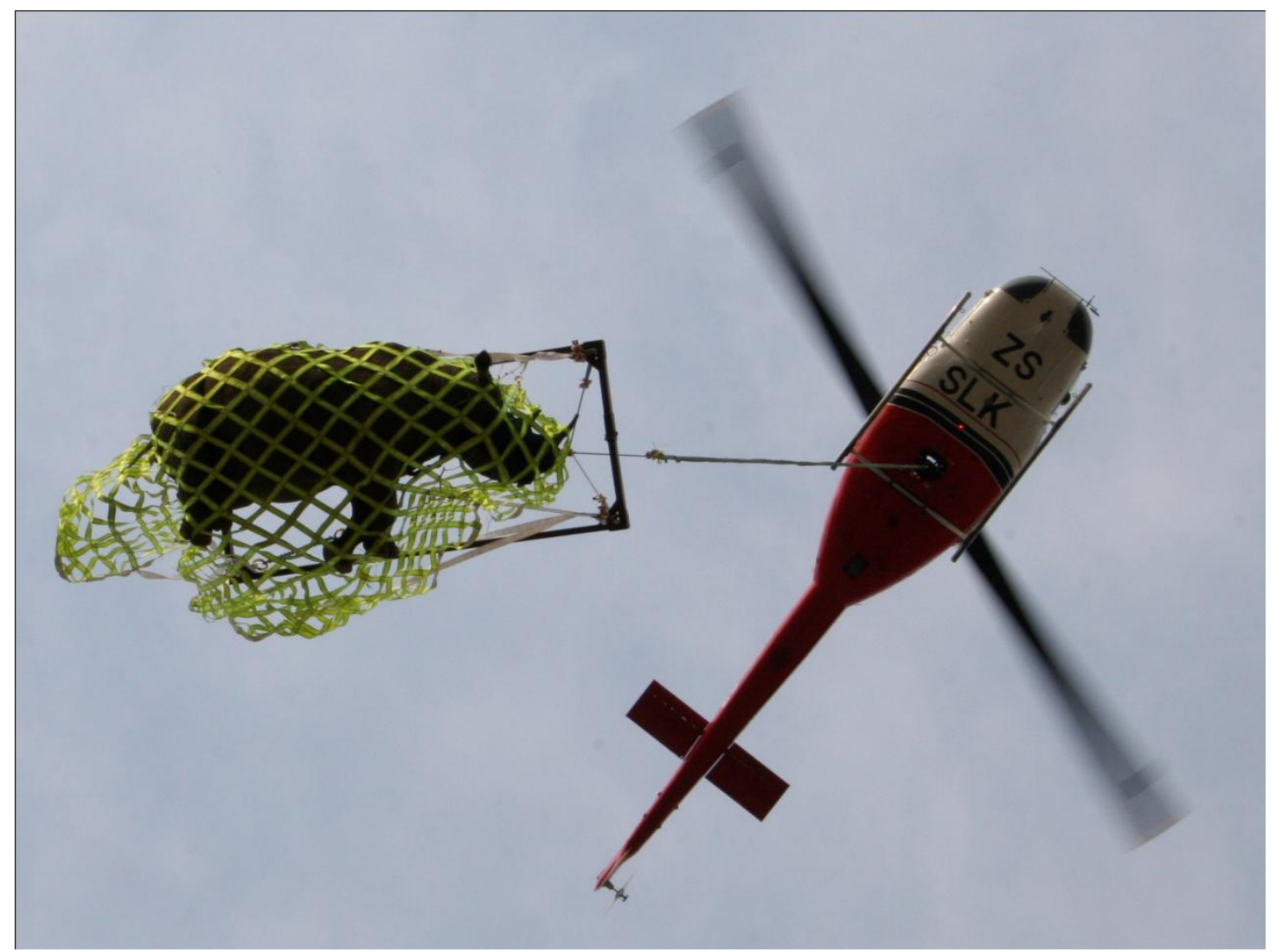

Photo by Rosalynn Anderson-Lederer 
Chapter III: Microsatellite DNA

\subsection{Abstract}

When preparing management plans based on genetic information, it is helpful to validate results with discrete tests to confirm outcomes. Mitochondrial DNA (mtDNA) combined with microsatellite DNA markers can assist managers in making conservation decisions based on understanding the genetic structure of a species. Previous studies indicated that the expected heterozygosity in the black rhino (Diceros bicornis minor) metapopulation of KwaZulu-Natal (KZN) including the source population of Hluhluwe-iMfolozi (HiP), South Africa is within the range of other large animals across Africa and no changes in management policy were necessary. However, recent mtDNA findings of one unique haplotype $(n=65)$ in the KZN metapopulation compared to six haplotypes $(\mathrm{n}=11)$ in native Zimbabwe $D . b$. minor suggest otherwise. I used 10 microsatellites and found that the KZN metapopulation was out of Hardy-Weinberg Equilibrium and showed excess homozygosity at five loci. I confirmed mtDNA findings that the South African metapopulation has lower genetic variation than the native Zimbabwe $D . b$. minor population indicating that current conservation plans might need to be modified to prevent further genetic decay. A loss of genetic diversity might be arrested by either (1) increasing population numbers to accommodate needed growth by expanding habitat and reserve sizes, or (2) carrying out a serial translocation scheme between the metapopulation of smaller populations including the source population Hluhluwe-iMfolozi and native D. b. minor from Zimbabwe to generate an artificially larger single population. Implementing these recommended changes could help reduce further genetic loss and maintain the levels of genetic variability 
in the HiP source and KZN metapopulation.

\subsection{Introduction}

Understanding the genetic structure of wild populations provides conservation managers with valuable insight into the design of management plans for reintroduction and supplementation. Levels of genetic variability are known to vary among populations, making it a perfect tool for determining the underlying structure of a natural population. While not always feasible, the level of genetic variability should be quantified using a range of DNA markers, and decisions should be based on corroborated results (Moritz 1994; Waits et al. 1998; Manceau et al. 1999). For example, recommended guidelines for the management of the Scandinavian brown bear (Ursus arctos) (Taberlet and Bouvet 1994; Waits et al. 2000) and western North American caribou (Rangifer tarandus) (Weckworth et al. 2012) are the consensus of mtDNA and microsatellite data.

Microsatellite and mtDNA are complimentary metrics for examining the genetic structure and the level of diversity of a populations (Toews and Brelsford 2012). MtDNA is maternally inherited and has one quarter of the effective size $\left(\mathrm{N}_{\mathrm{e}}\right)$ of a nuclear diploid locus, which makes it more sensitive to changes in population size. Microsatellite DNA markers, on the other hand, enable a better coverage of the genome and give a more precise estimate of the level of genetic variation in a population. Typically highly polymorphic, microsatellites are used extensively in genetic studies (Bruford and Wayne 1993; Jarne and Lagoda 1996; Forstmeier et al. 2012). 
African black rhino (Diceros bicornis) once ranged across the African continent in large numbers (Ashley et al. 1990; Lacombat 2005), but are now endangered (Emslie 2011). The genetic structure of the species has been extensively studied (ALLOYZMES: Merenlender et al. (1989); Ashley et al. (1990); Swart et al. (1994), MTDNA SEQUENCING: Brown and Houlden (2000); Muya et al. (2011); Anderson-Lederer et al. (2012) MICROSATELLITES: Brown and Houlden (1999); Cunningham et al. (1999); Garnier et al. (2001); Harley et al. (2005); Karsten et al. (2011); Muya et al. (2011)), yet confusion remains regarding subspecific nomenclature and the grouping of subspecies into 'ecotypes' (Zukowsky 1965; Groves 1967; du Toit 1986, 1987; Rookmaaker 1995, 2005).

Hluhluwe-iMfolozi Game Park (HiP) (Fig. 3.1) in the KwaZulu-Natal (KZN) province of South Africa has the largest remnant population of critically endangered (IUCN 2008) D. b. minor (n 220; Clinning et al. 2009). Successful translocations of D. b. minor from HiP to other KZN reserves began in 1962, later expanding to other South African provinces and African nations (e.g., Zimbabwe, Zambia, Swaziland) (Hitchins 1984; Emslie et al. 2009). While translocations have resulted in expansion and growth of the KZN D. b. minor metapopulation, the HiP source remains small, with no translocations back into the population, making it vulnerable to loss of genetic variation. Harley et al. (2005) and Karsten et al. (2011) demonstrated that $D$. b. minor subspecies had lower microsatellite variability than the other two subspecies (D. b. michaeli and D. b. bicornis). More specifically, Anderson-Lederer et al. (2012) established that the KZN metapopulation of D. $b$. minor are fixed for a single unique haplotype whereas six haplotypes $(\mathrm{n}=11)$ were 
identified in native Zimbabwe $D$. b. minor. The mtDNA results could be used to imply that genetic variability may be decreasing within HiP.

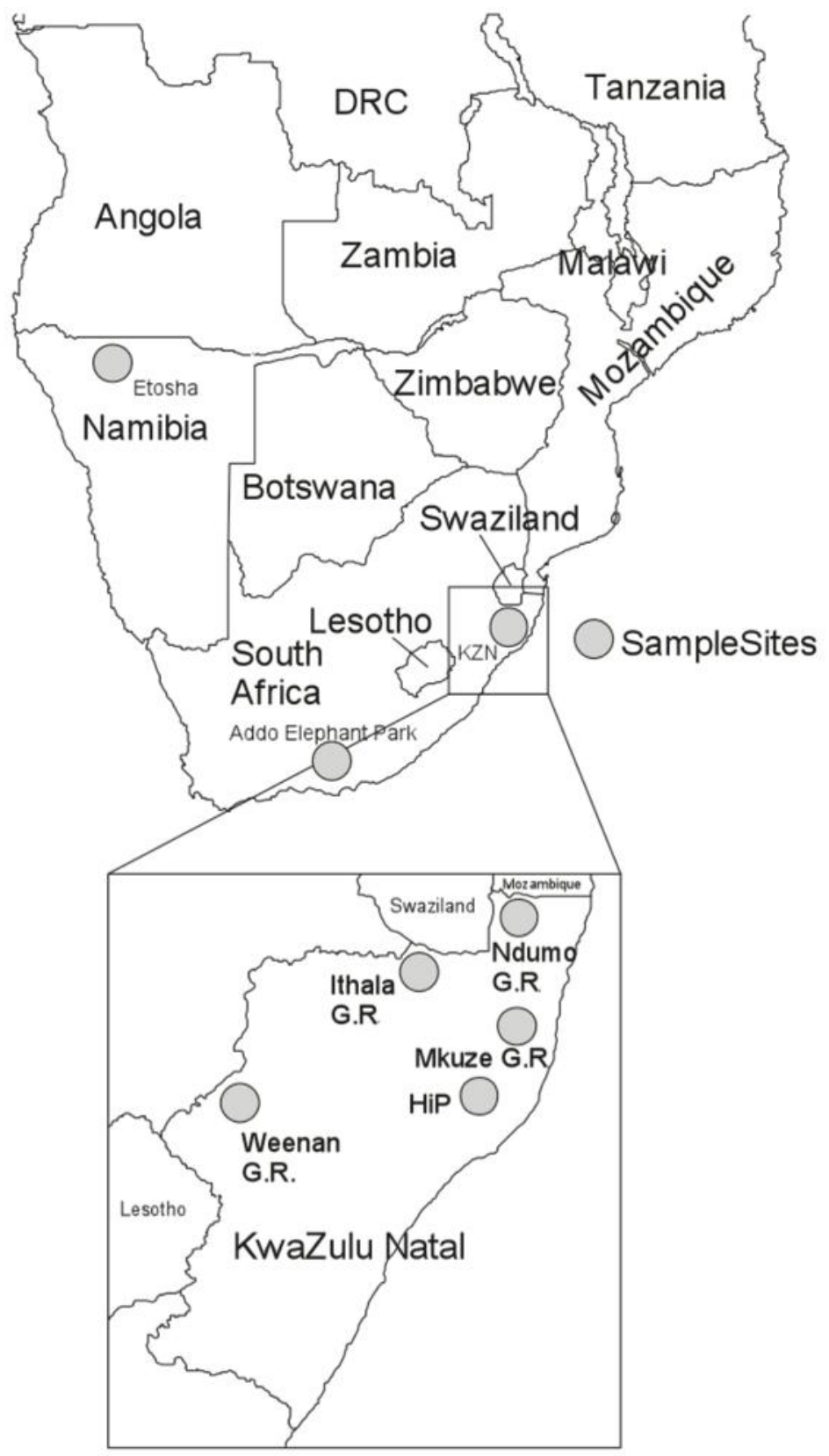

Figure 3.1: Map of southern Africa with sample sites indicated by circles. Inset of KwaZuluNatal with sample sites Hluhluwe-iMfolozi Game Park (HiP), Mkuze Game Reserve, Ndumo Game Reserve, Ithala Game Reserve and Weenan Game Reserve. 
Table 3.1: Characteristics of 10 microsatellite markers used to type 127 black rhinoceros and the number of alleles found in $118 \mathrm{KZN}$ D. b. minor

\begin{tabular}{|c|c|c|c|c|c|c|c|}
\hline $\begin{array}{c}\text { Locus } \\
\text { ID }\end{array}$ & Repeat Size & Primer pair sequence 5'-3' & Author & Tag & $\begin{array}{l}\text { Size } \\
\text { (bp) }\end{array}$ & $\begin{array}{l}\text { Annealing } \\
\text { Temp }\left(C^{\circ}\right)\end{array}$ & $\begin{array}{l}\text { No. of } \\
\text { alleles }\end{array}$ \\
\hline BR4 & $(\mathrm{CA}) 19$ & (F) CCC CTA AAT TCT AGG AAC AC & (Cunningham et al. 1999) & M-13 VIC & $124-146$ & 49 & 5 \\
\hline BR6 & $(\mathrm{CA}) 15$ & $\begin{array}{l}\text { (R) CCA AAG ACC ACC AGT AAT TC } \\
\text { (F) TCA TIT CTT TGT TCC CCA TAG CAC } \\
\text { (R) AGC AAT ATC CAC GAT ATG TGA AGG }\end{array}$ & " & M-13 VIC & $126-158$ & 51 & 4 \\
\hline BR17 & (AT)6(GT) 18 & $\begin{array}{l}\text { (F) ACT AGC CCT CCT TTC ATC AG } \\
\text { (R) GCA TAT TGT AAG TGC CCC AG }\end{array}$ & $"$ & Fluoro VIC & $123-135$ & 60 & 3 \\
\hline DB1 & $(\mathrm{CA}) 14$ & $\begin{array}{l}\text { (F) AGA TAA TAA TAG GAC CCT GCT CCC } \\
\text { (R) GAG GGT TTA TTG TGA ATG AGG C }\end{array}$ & (Brown \& Houlden 1999) & Fluoro FAM & $121-127$ & 60 & 2 \\
\hline DB5 & $(\mathrm{CA}) 13$ & $\begin{array}{l}\text { (F) GAC CCC CAT GTT CAC TGC } \\
\text { (R) AGG TCC ATC CAT TIT GTC CC }\end{array}$ & $"$ & Fluoro FAM & $185-204$ & 60 & 4 \\
\hline DB30 & $(\mathrm{CA}) 21$ & $\begin{array}{l}\text { (F) GCG ACT ATG ACA TAC AAC TAT CTA C } \\
\text { (R) GGT CA AGG ATT ATT CTG ACT AGC }\end{array}$ & $"$ & Fluoro VIC & $201-205$ & 64 & 4 \\
\hline DB44 & $(\mathrm{CA}) 4 \mathrm{G}(\mathrm{CA}) 16$ & $\begin{array}{l}\text { (F) GGT GGA ATG TCA AGT AGC GG } \\
\text { (R) CTT GTT GCC CCA TCC CTG }\end{array}$ & $"$ & M-13 VIC & $170-184$ & 64 & 1 \\
\hline DB49 & $(\mathrm{CA}) 14$ & $\begin{array}{l}\text { (F) GTC AGG CAT TGG CAG CAA G } \\
\text { (R) CAG GGT AAG TGG GGG TGC }\end{array}$ & $"$ & Fluoro FAM & $152-162$ & 64 & 4 \\
\hline DB52 & $(\mathrm{CA}) 21$ & $\begin{array}{l}\text { (F) CAT GTG AAA TGG ACC GTC AGG } \\
\text { (R) ATT TCT GGG AAG GGG CAG G }\end{array}$ & $"$ & Fluoro FAM & $210-220$ & 64 & 3 \\
\hline DB66 & $(\mathrm{CA}) 7 \mathrm{TA}(\mathrm{CA}) 16$ & (F) CCA GGT GAA GGG TCT TAT TAT TAG C & $"$ & Fluoro FAM & $187-205$ & 58 & 3 \\
\hline
\end{tabular}

In light of recent mtDNA findings, re-examination of the levels of variation using microsatellite markers in the KZN D. b. minor metapopulation and more specifically the HiP source population is required. The aim of this study was to use ten microsatellite DNA markers (Table 3.1) to investigate levels of heterozygosity and allelic diversity in HiP D. b. minor. I then compared these results to the KZN metapopulation and previously published microsatellite and sequenced mtDNA control region data. I used the results to make recommendations for translocation, reintroduction and supplementation for KZN's D. b. minor source and metapopulation.

\subsection{Methods}

\section{Sampling}

Samples of blood and/or ear tissue were collected from a total of 127 individuals of D.b. minor in the KZN province in South Africa, D .b. michaeli in Addo Elephant 
National Park and D. b. bicornis in Namibia's Northern Region (Table 3.2). The samples were acquired opportunistically during routine translocation and ear notching (for identification) events from 2002 to 2009. Blood and tissue samples were stored in cryovials containing $1 \mathrm{~mL}$ of DMSO/EDTA/Tris/salt solution (Seutin et al. 1991) or RNAlater ${ }^{\circledR}$ Solution (Life Technologies).

Table 3.2: Subspecies of Diceros bicornis and corresponding populations and sample size $(\mathrm{N})$. All D. b. minor samples are from the KwaZuluNatal metapopulation

\begin{tabular}{llc}
\hline Subspecies & Population & $\mathrm{N}$ \\
\hline D. b. minor & Eastern Shores & 2 \\
& Hluhluwe-iMfolozi Park & 97 \\
& Ithala Game Reserve & 10 \\
& Johannesburg Zoo & 1 \\
& Mkuze Game Reserve & 6 \\
& Ndumo Game Reserve & 1 \\
& Tembe Elephant Park & 1 \\
& KZN Populations Combined & $\mathbf{1 1 8}$ \\
D. b. & Addo Elephant Park, South & 3 \\
michaeli & Africa & \\
D. b. bicornis & Waterberg National Park, & 6 \\
& Namibia
\end{tabular}

Genetic Analyses

I extracted genomic DNA using DNeasy kits (Qiagen Inc.) following the manufacturer's recommended protocol. Ten polymorphic microsatellite loci (Table 3.1) reported by Cunningham et al. (1999) and Brown and Houlden (1999) were chosen based on their reliability and were amplified by polymerase chain reaction 
(PCR). One primer from each pair was labeled using the M-13 tag methodology (Schuelke 2000) or directly labeled with a fluorescent dye (6-FAM or VIC, Invitrogen). The resultant PCR products were analysed on a 3730 automated sequencer using the GS-500 LIZ size standard and the GENESCAN software (Applied Biosystems). Samples that did not amplify for all loci were removed from the data set. Alleles were visualized and analysed using GENEMAPPER software ver. 3.7 (Applied Biosystems), then results were then confirmed with GENEMARKER software (Softgenetics). MicRoCHECKER ver. 2.2.0.3 (Van Oosterhout, 2004) assessed possible reasons for deviation of HWE, which include null alleles (one or more alleles that fail to amplify during PCR), large allele dropout (small alleles amplify better than large alleles) and scoring errors due to stutter (slight changes that that occur in the allele sizes during PCR).

Hardy-Weinberg Equilibrium (HWE) (non-random association of alleles within diploid individuals), linkage disequilibrium (non-random association of alleles at different loci) and heterozygote excess and deficiency were estimated using GenePoP ver. 1.2 (Raymond and Rousset 1995; Rousset 2008). The Markov Chain parameters for the locus-by-locus pair-wise tests for gametic disequilibrium utilized 1000 dememorizations, 100 batches, and 5000 iterations per batch. Statistical significance (P-value) was corrected for multiple testing using the False Discovery Rate (FDR) procedure (Benjamini and Hochberg 1995). ARLEQUIN ver. 3.5 (Excoffier et al. 2005) was used to assess the level of population differentiation between the three subspecies based on Wright's (1965) pairwise $F_{\mathrm{ST}}$, which is derived from the variances of allele frequency and Slatkin's (1995) $R_{\mathrm{ST}}$, which 
calculates the fraction of total variance of allele size that exists between populations (Balloux and Lugon-Moulin 2002). The level of significance was assessed at 1000 permutations. I also examined $D_{\text {est }}($ Jost 2008), which is an estimate for actual differentiation, with SMOGD ver. 1.2.5 (Crawford 2010) using 1000 bootstrap replicates. Allelic richness ( $A r$, a measure of the number of alleles corrected for different sample sizes) was calculated for each subspecies and each loci with HP-Rare ver. 1.0 (Kalinowski 2005). Inbreeding coefficient $F_{\text {IS }}$ was analysed using FsTAT (Goudet 1995).

\section{Genetic Structure}

STRUCTURE ver. 2.3.3 (Pritchard et al. 2000), a Bayesian model-based clustering software implementing the Markov Chain Monte Carlo (MCMC) method was used to identify distinct genetic patterns in the subspecies populations and designate individuals to one or more genetic clusters (K). One potential criticism of STRUCTURE is that the output of this programme can be difficult to interpret when levels of population structure are low. Therefore data was analysed using the LOCPRIOR setting within STRUCTURE, which uses the sampling locations of individuals to assist the clustering process, thereby generating more accurate estimates of $K$ (Hubisz et al. 2009). Ten STRUCTURE runs for each value of $K$ were carried out (one to five for the $D$. bicornis subspecies and one to eight for the KZN $D$. $b$. minor metapopulation) for 1000000 iterations and a burn-in time of 100000 iterations for both data sets. Since the three subspecies sampled may have mixed ancestry, admixture ancestry model was chosen (Pritchard et al. 2007). Allele frequencies were correlated among populations and assumed different values of $F_{\mathrm{ST}}$ for the different 
subpopulations. StRUCTURE HARVESTER ver. A.1 (Earl and vonHoldt 2012) which applies the Evanno method (Evanno et al. 2005) was used to visualize STRUCTURE output.

\section{Testing for a Genetic Bottleneck}

I examined signatures of a reduction in population size using BOTTLENECK ver. 1.2.2 (Cornuet and Luikart 1996; Luikart et al. 1998; Piry et al. 1999). This analysis is designed to detect a recent bottleneck occurring within the past $2 \mathrm{~N}_{\mathrm{e}}-4 \mathrm{~N}_{\mathrm{e}}$ generations, assuming the populations were in mutation-drift equilibrium. Piry et al. (1999) recommends the Wilcoxon two-tailed sign-rank test within BoTTLENECK, which accounts for both heterozygosity excess and deficiency for data with less than 20 polymorphic loci and where effective population sizes may have remained constant for long periods of time. The three mutation models within the Wilcoxon test are the infinite allele model (IAM), the stepwise mutation model (SMM) and the two-phase model (TPM); microsatellite mutation was set at 95\% single-step mutation rate and 5\% multiple step mutation along with the variance among multiple steps of 12 (Piry et al. 1999). BoTTLENECK also tested for a mode-shift of the allele frequency distribution, because when a population has recently been through a bottleneck, rare alleles are typically lost causing a distortion in allele frequencies at selectively neutral loci (Luikart et al. 1998). Since Harley et al. (2005) found a significant departure from HWE and a slight overall homozygous excess in their mixed Zimbabwe/South Africa samples of D. b. minor (n=46), I also checked for a bottleneck using the Garza-Williamson index or M-ratio (Garza and Williamson 2001) implemented in ARLEQUIN ver. 3.5 (Excoffier et al. 2005). The M-ratio (M = 
$k / r$ where $k=$ number of alleles and $r=$ overall range in fragment sizes) can identify a bottleneck even when data is out of equilibrium and a modified M-ratio is used if any of the loci are monomorphic.

\subsection{Results}

All ten microsatellite loci were amplified successfully. There was evidence of null alleles at loci DB5 and DB1 in the KZN D. b. minor samples ( $\mathrm{n}=118)$. The two loci were removed for KZN metapopulation comparisons, but neither loci was excluded from the data set for comparison between the three subspecies, since null alleles were not present in either D. b. micheali or D. b. bicornis. Results for D. b. bicornis samples $(n=6)$ were monomorphic at two loci and there was no evidence for scoring error due to stuttering, large allele dropout or null alleles. The D. b. michaeli samples $(n=3)$ had too few alleles at each locus to perform the same tests. No significant linkage disequilibrium was observed for any pairs of loci after FDR correction.

The KZN D. b. minor population ( $\mathrm{n}=118)$ was not in HWE. Five of the ten loci showed deviation from HWE (DB30, DB1, BR17, DB5 and DB52) (Table 3.3) and locus DB44 was monomorphic. Three loci showed deviation from HWE after an FDR correction (DB30, DB5 and DB52). The D. b. michaeli samples (n=3) were in HWE, but were monomorphic at two loci (DB1 \& DB44) (Table 3.3). The $D . b$. bicornis samples $(\mathrm{n}=6)$ were in HWE but monomorphic at one locus (BR4) (Table 3.3). 
Table 3.3: $H_{\mathrm{e}}$ and $H_{\mathrm{o}}$ per locus, per subspecies with associated P-values for $D$. b. bicornis $(\mathrm{n}=6), D . b$. michaeli $(\mathrm{n}=3), \mathrm{KZN} D . b$. minor $(\mathrm{n}=$ 118 ) and native Zimbabwe $D . b$. minor $(n=35)$. Zimbabwe data are from Garnier et al. (2001). Bold data highlight regional variations between $D$. $b$. minor subspecies. (M: monomorphic, no value)

\begin{tabular}{|c|c|c|c|c|c|c|c|c|c|c|c|}
\hline \multirow[t]{2}{*}{ Locus } & \multicolumn{3}{|c|}{ D. b. bicornis } & \multicolumn{3}{|c|}{ D. b. michaeli } & \multicolumn{3}{|c|}{$\begin{array}{c}\mathrm{KZN} \\
\text { D. b. minor }\end{array}$} & \multicolumn{2}{|c|}{$\begin{array}{l}\text { Zimbabwe } \\
\text { D. b. minor }\end{array}$} \\
\hline & $H_{\mathrm{e}}$ & $H_{\mathrm{o}}$ & P-value & $H_{\mathrm{e}}$ & $H_{0}$ & P-value & $H_{\mathrm{e}}$ & $H_{\mathrm{o}}$ & P-value & $H_{\mathrm{e}}$ & $H_{0}$ \\
\hline DB30 & 0.62 & 1.00 & 1.00 & 0.33 & 0.33 & - & 0.63 & 0.60 & 0.002 & \multicolumn{2}{|c|}{ not tested } \\
\hline DB1 & 0.59 & 0.67 & 0.76 & $M$ & - & - & 0.33 & 0.25 & 0.009 & 0.46 & 0.69 \\
\hline BR17 & 0.41 & 0.50 & 1.00 & 0.60 & 0.33 & 0.20 & 0.20 & 0.17 & 0.029 & 0.61 & 0.69 \\
\hline DB66 & 0.74 & 0.83 & 0.19 & 0.93 & 1.00 & 1.00 & 0.64 & 0.66 & 0.598 & 0.72 & 0.91 \\
\hline DB5 & 0.67 & 1.00 & 1.00 & 0.73 & 0.33 & 0.21 & 0.64 & 0.52 & 0.001 & 0.58 & 0.69 \\
\hline BR6 & 0.32 & 0.33 & 1.00 & 0.87 & 0.67 & 0.33 & 0.55 & 0.56 & 0.768 & 0.80 & 0.81 \\
\hline BR44 & 0.68 & 0.33 & 0.05 & $M$ & - & - & M & - & - & 0.47 & 0.47 \\
\hline BR4 & $M$ & - & - & 0.87 & 1.00 & 1.00 & 0.66 & 0.58 & 0.109 & 0.60 & 0.69 \\
\hline DB49 & 0.64 & 0.20 & 0.50 & 0.73 & 1.00 & 1.00 & 0.55 & 0.58 & 0.288 & 0.70 & 0.81 \\
\hline DB52 & 0.53 & 0.50 & 0.76 & 0.80 & 0.67 & 0.46 & 0.49 & 0.50 & 0.003 & 0.77 & 0.91 \\
\hline mean & 0.52 & & & 0.59 & & & 0.47 & & & 0.62 & \\
\hline
\end{tabular}

The $F_{\mathrm{ST}}$ results indicated differentiation between the three subspecies over the 10 microsatellite loci examined with values ranging from 0.091 - 0.20 (Table 3.4). $F_{\text {ST }}$ results for the three largest sample sets within the KZN metapopulation (HiP, Mkuze \& Ithala) indicated very little differentiation with the KZN samples with values between $0.001-0.03$ (Table 3.4). $R_{\text {ST }}$ results for the three subspecies were lower than those reported for $F_{\mathrm{ST}}$ and ranged from $0.02-0.10$ (Table 3.4) suggesting lower genetic differentiation between the subspecies. Allelic differentiation as expressed by $D_{\text {est }}$ was minimal between the three subspecies ranging between 0.05 and 0.10 (Jost 2009)(Table 3.4). 
Table 3.4: A) Pairwise $F_{\mathrm{ST}}$ values B) Pairwise $R_{\mathrm{ST}}$ values C) Pairwise $D_{\text {est }}$ values for $D$. bicornis

\begin{tabular}{|c|c|c|}
\hline A) $F_{\mathrm{ST}}$ & D. b. michaeli & D. b. minor \\
\hline D. b. minor & $0.09094(p=0.01802 \pm 0.0121)$ & \\
\hline D. b. bicornis & $0.20116(p=0.00301 \pm 0.0091)$ & $0.19101(p=0.000 \pm 0.000)$ \\
\hline$F_{\mathrm{ST}}$ & Mkuze & $\mathrm{HiP}$ \\
\hline $\mathrm{HiP}$ & $0.002044(p=0.11712 \pm 0.0237)$ & \\
\hline Ithala & $0.001111(p=0.30631 \pm 0.0388)$ & $0.02846(p=0.02703 \pm 0.0194)$ \\
\hline B) $R_{\mathrm{ST}}$ & D. b. michaeli & D. b. minor \\
\hline D. b. minor & $0.10011(p=0.09009 \pm 0.0271)$ & \\
\hline D. b. bicornis & $0.09305(p=0.01802 \pm 0.0121)$ & $0.02104(p=0.10811 \pm 0.0353)$ \\
\hline C) $D_{\text {est }}$ & D. b. michaeli D. b. minor & \\
\hline D. b. minor & 0.04675 & \\
\hline D. b. bicornis & 0.09656 & \\
\hline
\end{tabular}

$H_{\mathrm{E}}$ averaged over all 10 loci for the three subspecies were between $0.47 \pm$ 0.22 and $0.59 \pm 0.35$, while $H_{0}$ were between $0.49 \pm 0.17$ and $0.67 \pm 0.31$ (Table 3.5). $H_{\mathrm{E}}$ and $H_{0}$ for KZN source populations were as follows: $\mathrm{HiP}=0.45 \pm 0.24$ and $0.48 \pm 0.23$; Mkuze; $0.46 \pm 0.22$ and $0.49 \pm 0.25$ respectively. When loci DB1 and DB5 were removed from the KZN souce populations for anaylsis (HiP and Mkuze) $H_{\mathrm{E}}$ was only slightly changed: $\mathrm{HiP}=0.45 \pm 0.25$; Mkuzi $=0.43 \pm 0.23 . H_{\mathrm{E}}$ and $H_{\mathrm{O}}$ for the D. b. minor Zimbabwe data published by Garnier et al. (2001) was $0.62 \pm 0.13$ and $0.72 \pm 0.13$ respectively (Table 3.5). Ar for the three subspecies was between 2.18 and 2.90 (Table 3.5) while $F_{\text {IS }}$ was between $-0.032 \pm 0.46$ and $0.054 \pm 0.12$ (Table 3.5). 
Table 3.5: Microsatellite results from this study, Harley et al. 2005, Karsten et al. 2011, Garnier et al. 2001, Muya et al. 2011 and Van Coeverden de Groot et al. 2011. $\mathrm{N}$, number of individuals analysed; $H_{\mathrm{E}}$, expected heterozygosity (for polymorphic loci); $H_{0}$, observed heterozygosity (for polymorphic loci); SD,

standard deviation; $A r$, allelic richness (averaged over loci); $F_{\mathrm{IS}}$, inbreeding coefficient.

\begin{tabular}{|c|c|c|c|c|c|c|c|c|c|c|c|}
\hline Study & Microsatellites Used & Country & Subspecies & $\bar{N}$ & $\overline{H_{\mathrm{E}}}$ & SD & $\mathrm{H}_{\mathrm{O}}$ & SD & $A r$ & $\overline{F_{\mathrm{IS}}}$ & SD \\
\hline \multirow[t]{3}{*}{ This study } & BR4, BR6, BR17 & South Africa & D. b. minor & 118 & 0.47 & \pm 0.22 & 0.49 & \pm 0.17 & 2.18 & 0.054 & \pm 0.12 \\
\hline & (Cunningham et al. 1999) & Namibia & D. b. bicornis & 6 & 0.52 & \pm 0.22 & 0.60 & \pm 0.30 & 2.34 & -0.032 & \pm 0.46 \\
\hline & $\begin{array}{l}\text { DB1, DB5, DB30, DB44, DB49, } \\
\text { DB52, DB66 (Brown \& Houlden 1999) }\end{array}$ & South Africa & D. b. michaeli & 3 & 0.59 & \pm 0.35 & 0.67 & \pm 0.31 & 2.90 & 0.111 & \pm 0.39 \\
\hline \multirow[t]{3}{*}{ Harley et al. 2005} & BR4, BR6, BR17 & South Africa \& Zimbabwe & D. b. minor & 47 & 0.46 & - & 0.44 & - & & - & - \\
\hline & (Cunningham et al. 1999) & Namibia & D. b. bicornis & 53 & 0.51 & - & 0.52 & - & & - & - \\
\hline & $\begin{array}{l}\text { DB1, DB14, DB44, DB49, } \\
\text { DB52, DB66 (Brown \& Houlden 1999) }\end{array}$ & South Africa & D. b. michaeli & 19 & 0.68 & - & 0.73 & - & & - & - \\
\hline \multirow{5}{*}{ Karsten et al. 2011} & BR4, BR6, BR17 & South Africa \& Zimbabwe & D. b. minor & 77 & 0.44 & - & 0.38 & - & & 0.14 & - \\
\hline & (Cunningham et al. 1999) & Namibia & D. b. bicornis & 4 & 0.43 & - & 0.46 & - & & 0.09 & - \\
\hline & DB1, DB14, DB49, DB66 & South Africa \& Tanzania & D. b. michaeli & 4 & 0.54 & - & 0.54 & - & & 0.16 & - \\
\hline & (Brown \& Houlden 1999) & & & & & & & & & & \\
\hline & AY606078, AY606080, AY606083 & & & & & & & & & & \\
\hline \multirow[t]{4}{*}{ Garnier et al. 2001} & BR4, BR6, BR17 & Zimbabwe & D. b. minor & 35 & 0.616 & - & 0.726 & - & & - & - \\
\hline & (Cunningham et al. 1999) & & & & & & & & & & \\
\hline & DB1，DB5，DB23，DB44，DB49, & & & & & & & & & & \\
\hline & DB52, DB66 (Brown \& Houlden 1999) & & & & & & & & & & \\
\hline \multirow[t]{4}{*}{ Muya et al. 2011} & BR4, BR6, BR17 & Kenya & D. b. michaeli & 145 & 0.69 & \pm 0.03 & 0.70 & \pm 0.09 & & 0.046 & \pm 0.09 \\
\hline & (Cunningham et al. 1999) & & & & & & & & & & \\
\hline & DB1, DB5, DB30, DB44, & & & & & & & & & & \\
\hline & DB52, DB66 (Brown \& Houlden 1999) & & & & & & & & & & \\
\hline \multirow[t]{5}{*}{$\begin{array}{l}\text { Van Coeverden de } \\
\text { Groot et al. } 2011\end{array}$} & $\begin{array}{l}\text { DB1, DB44, DB52, DB66 } \\
\text { (Brown \& Houlden 1999) }\end{array}$ & Namibia & D. b. bicornis & 144 & 0.51 & - & 0.52 & - & & - & - \\
\hline & BR4, BR6, BR17 & & & & & & & & & & \\
\hline & (Cunningham et al. 1999) & & & & & & & & & & \\
\hline & BIRh2B，BIrh37D & & & & & & & & & & \\
\hline & (Van Coeverd & & & & & & & & & & \\
\hline
\end{tabular}




\section{Genetic Structure}

The STRUCTURE analysis indicated, the maximum mean log likelihood value of -2094.54 for the three D. bicornis subspecies was most likely $K=2(-2195.10$ for $K=1$ and -2106.98 for $K=3$ ). Utilizing the Evanno method (Evanno et al. 2005), the highest value of $\Delta \mathrm{K}$ also indicated that the number of clusters was likely $K=$ 2 (Fig. 3.2A). The maximum mean log likelihood value of -1776.88 indicated that the number of clusters for three largest populations in KZN (HiP, Mkuze and Ithala) was $K=1(-1808.80$ for $K=2)$, utilizing the Evanno method (Evanno et al. 2005), the highest value of $\Delta \mathrm{K}$ inferred that the number of clusters was $K=2$ (Fig. 3.2B). However, $\Delta \mathrm{K}$ is based on the second order rate of change with respect to the likelihood associated with $\mathrm{K}$ and is not a suitable method for detecting if the true $K$ of a population is $K=1$ (Evanno et al. 2005).

\section{Bottleneck}

Wilcoxon two-tailed sign-rank test for bottleneck gave conflicting results. $D . b$. minor showed a classic L-shaped allele frequency distribution, and both the SMM and TPM were both in mutation-drift equilibrium (no bottleneck detected), however the IAM was out of equilibrium (indicating bottleneck). The D. b. michaeli and D. b. bicornis populations could not be assessed, as there were too few samples for the Wilcoxon and mode-shift tests. Modified M-ration (Excoffier et al. 2005) results indicated that all three populations have been through significant bottlenecks: D. b. minor 0.26494 , D. b. michaeli 0.22761 and D. b. bicornis 0.25238 (results $<0.7$ indicate bottleneck; Excoffier et al. 2005). Not all of the loci in this study were polymorphic, so the results of the modified M-ratio were reported. 
A

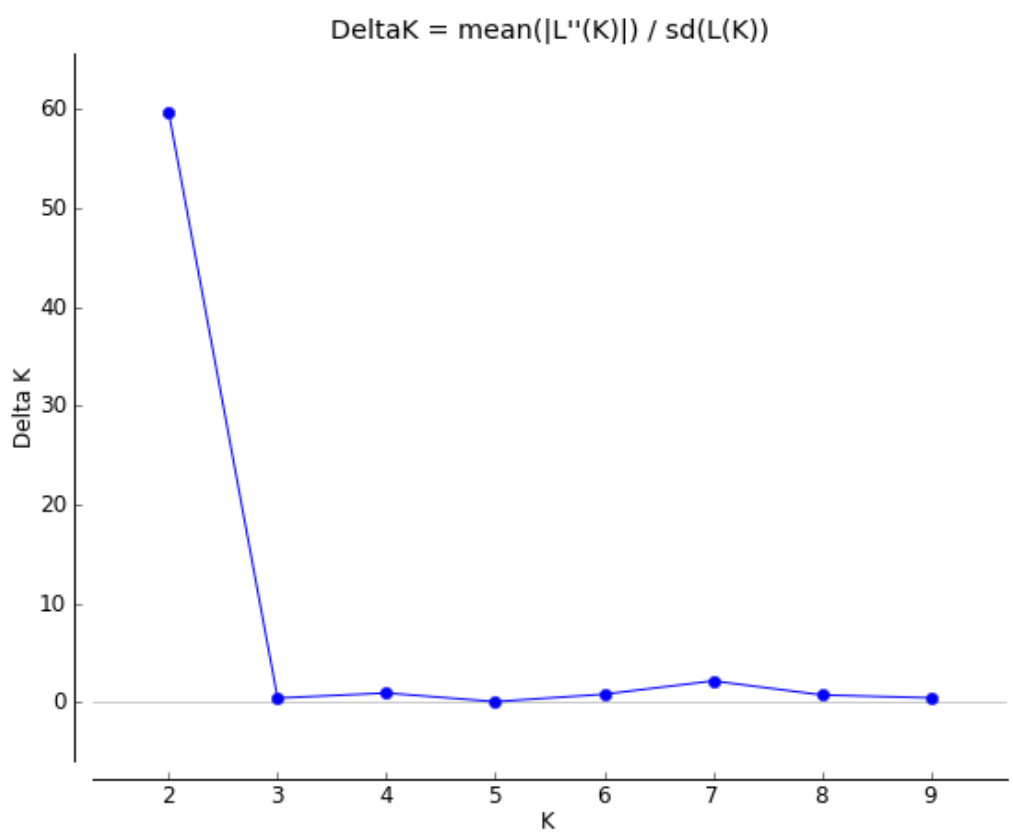

B

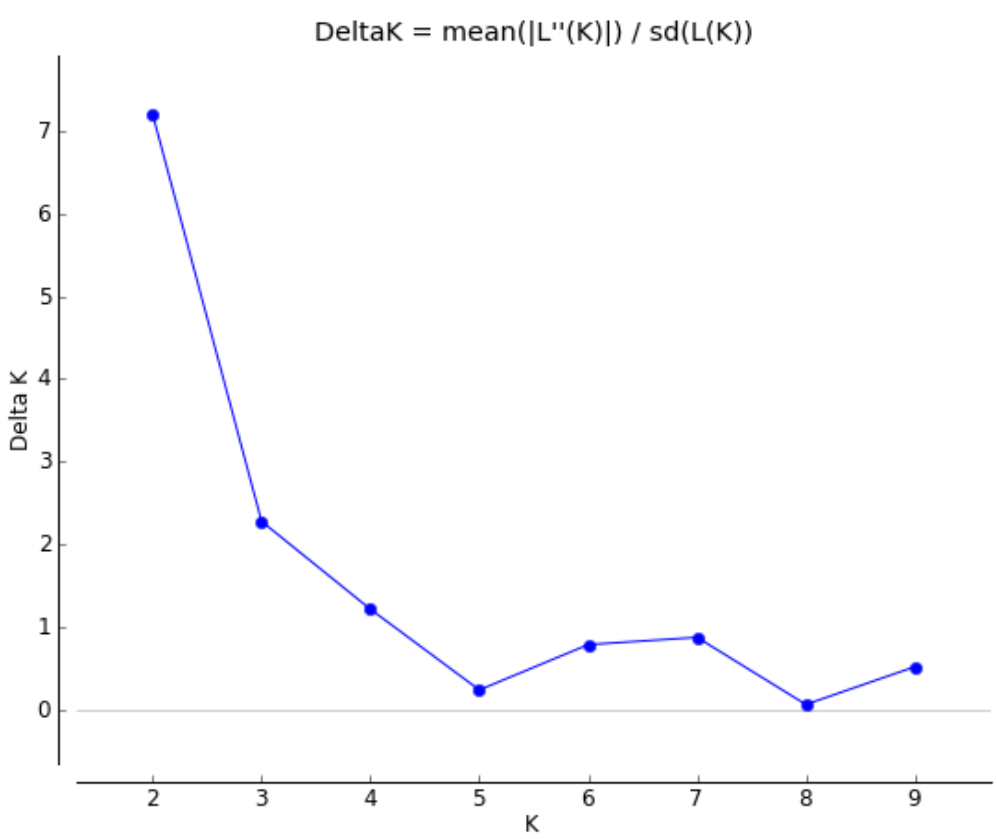

Figure 3.2: STRUCTURE population genetic structure output for D. bicornis.

Determination of the number of clusters using $\Delta K$ for values of $K$ from 1 to 10 . A) Three D. bicornis subspecies, $\mathrm{K}=2$. B) $\mathrm{KZN}$ sample set, $\mathrm{K}=2$. 


\subsection{Discussion}

The KZN D. b. minor population had an excess of homozygotes at five out of ten microsatellite loci and deviated from HWE expectations. This was consistent (excess homozygotes) with those reported by Harley et al. (2005) whose $D . b$. minor samples were a combination of both South Africa and Zimbabwe individuals. Examination of eight out of 10 of the same microsatllite loci (Table 3.3) as Harley et al. (2005) and $~ 81 \%$ of their samples collected from KZN are probable contributing factors to the similar outcomes.

The $R_{\mathrm{ST}}$ values for this data set should be viewed with skepticism since $R_{\text {ST }}$ does not perform well with a small sample size $(\leq 10)$ or a small number of loci $(\leq 20)$, as is the case with this study (Gaggiotti et al. 1999). In addition a histogram of allele sizes for the largest population (HiP; not shown) revealed multiple peaks, indicating deviation from the assumption of stepwise mutation, further indicating that $R_{\mathrm{ST}}$ is probably not a suitable measure for this data set. Although based on the IAM, Fst outperforms $R_{\mathrm{ST}}$ in cases such as this where sample sizes are small (Gaggiotti et al. 1999). Fst and $D_{\text {est }}$ results suggest differentiation at the subspecies level, but very low differentiation at the KZN metapopulation level.

Identifying the cause of homozygote excess and departure from HWE may be difficult. The most commonly reported reasons a population can have excess homozygotes at microsatellite loci include scoring errors, the presence of null (non-amplifying) alleles (Pemberton et al. 1995), sampling more than one population (i.e., the Wahlund effect) and inbreeding (Castric et al. 2002). The possibility of scoring errors was elimination by MiCROCHECKER (Van Oosterhout, 2004). Samples that did not amplify for all loci were removed from the analysis. 
All remaining individuals in the data set amplified for all loci, indicating that there were no homozygotes for a null allele. This implies that the null alleles were only present at a low frequency and should not have significantly contributed to deviation from HWE.

Departure from HWE may also be present in a population with a low number of effective breeders (reproductive skew) (Luikart and Cornuet 1999). Garnier et al. (2001) found a high reproductive skew ( 53\%) in Zimbabwe $D . b$. minor that might be related to spatial distribution and linked to variations in fertility levels in each sex. A reproductive skew would affect the level of genetic vatiation in a small population more than it would in a larger population, especially if only a small number of males (as was the case in Zimbabwe black rhino) are contributing to reproductive output (Garnier et al. 2001).

STRUCTURE results suggest no significant population subdivision between the three subspecies and less within the KZN metapopulation. However, Rodriguez-Ramilo and Wang (2012) advised using caution interpreting output from STRUCTURE. Closely related individuals should be removed from datasets before conducting analyses, otherwise Hardy-Weinberg and linkage disequilibrium may skew results of genetic structure of the population. Unfortunately, there was no way of controlling the samples for this parameter since no pedigrees or studbooks are kept on the wild populations. I do however, have more confidence in the KZN STRUCTURE results than for the subspecies since the subspecies sample size for $D . b$. michaeli was only $\mathrm{n}=3$.

The indeterminate $D$. $b$. minor BOTTLENECK results might be attributed to testing less than 20 loci. Increasing the number of loci may provide discernable results, but since BOTTLENECK was designed to detect a recent bottleneck within 
the past $2 \mathrm{~N}_{\mathrm{e}}-4 \mathrm{~N}_{\mathrm{e}}$ generations, the IAM results are likely indicative of a bottleneck occurring more than $4 \mathrm{~N}_{\mathrm{e}}$ generations ago.

\section{Loss of Genetic Variability within HiP}

Reductions in microsatellite variation and bottleneck signature were not unexpected given that black rhino across Africa suffered rapid geographic and population size declines over the last century. However, the genetic variation of the KZN D. b. minor was significantly lower than that of the native Zimbabwe $D$. b. minor (KZN: $H_{\mathrm{E}} 0.47 \pm 0.22$; Zimbabwe: $H_{\mathrm{E}} 0.62 \pm 0.13$ ) (Table $3.3 \& 3.5$ ), which could be a consequence of low population numbers persisting for many generations (Harley et al. 2005). Although the use of translocations of $D . b$. minor between game reserves in KZN has aided in the retention of current levels of genetic variation (Karsten et al. 2011), there are no translocations of $D$. b. minor into the HiP source population. Lower microsatellite variability coupled with a fixed mtDNA haplotype (Anderson-Lederer et al. 2012), especially in HiP may signal a need for management to intervene to prevent further genetic decay within this valuable source population. If the level of genetic variability continues to decline without intervention (e.g. genetic rescue), it could lead to a reduction in adaptability (evolutionary potential) and increase the risk of inbreeding depression in HiP (Lacy 1987b; Burger and Lynch 1995). This has occurred in other species such as the black-footed rock-wallaby island populations (BFRW) (Petrogale lateralis) (Eldridge et al. 1999) and harbour seals (Phoca vitulina) (Coltman et al. 1998).

Swart and Ferguson (1997) speculated that native Zimbabwe black rhino populations were the only D. b. minor to retain pre-bottleneck levels of genetic 
variation. It is unclear why the small native Zimbawe populations have retained genetic variation through a severe bottleneck while KZN black rhino have not. To prevent a further loss of genetic variation and increasing risk of inbreeding depression within KZN but specifically $\mathrm{HiP}$, the following steps could be taken: (1) rapidly increasing population numbers by increasing reserve sizes or (2) serial translocations amongst the KZN metapopulation reserves, including back into the $\mathrm{HiP}$ source population, perhaps including replenishment using native Zimbabwe D. b. minor.

\section{Population Increases through Land Acquisitions and Serial Translocations}

The recommendation by Emslie (2001) for rapid growth as a buffer against black rhino poaching would also apply to precluding the effects of low genetic variability. Avoidance of a loss of allelic variation through rapid expansion has been documented in other animals. Despite only 13 European rabbits (Oryctolagus cuniculus) being imported to Australia in 1859 , there was no significant genetic difference between the contemporary introduced rabbit population and the population of European rabbits in France (Zenger et al. 2003). Researchers suspect that the initial population did not experience a decrease in levels of genetic variation because at no time were there enough generations at small sizes to lose significant diversity.

While HiP could increase in size by connecting adjacent reserves in northern KZN, the area of land in question may not be sufficiently large enough for increasing population numbers to levels necessary to arrest the loss of genetic variation. Mkuze Game Reserve (MGR) is connected via a corridor to Greater St. Lucia Wetland Park that extends along the east coast from Kosi Bay 
south to Mapelane encompassing Eastern Shores (one of this study's sample sites, Figure 3.4). Connecting MGR (including Greater St. Lucia Wetland Park from Sodwana National Park south to Mapelane) with close neighbours Phinda, Thanda, and founder populations established by the Black Rhino Range Expansion Project (Pongola, Munyawan and Zululand Game Reserves) would create an area that is very roughly $2,806 \mathrm{~km}^{2}$. Adding HiP $(\sim 40 \mathrm{~km}$ away from Zululand Game Reserves) into the conglomeration of reserves by establishing a corridor through existing subtropical fruit and sugarcane fields would increase the area to $\sim 3,745 \mathrm{~km}^{2}$. Using the average black rhino $/ \mathrm{km}^{2}$ of 0.22 for northern KwaZulu-Natal reserves (Adcock, K. pers. comm.) the approximate carrying capacity (CC) for an area that size would be about 824 , almost doubling the 430 CC for HiP. Procuring enough land for black rhino management to increase $\mathrm{HiP}$ to a size that would allow for rapid growth is unrealistic (Goodman 2001). A compromise to increasing land area of HiP may be found in serial translocations between $\mathrm{HiP}$ and other reserves to replicate immigration and emigration for each reserve. Translocations from HiP have been used as a successful black rhino management tool for KZN black rhinos since 1962 (Hitchins 1984; Hall-Martin and Knight 1994; Emslie et al. 2009). Rhinos moved back to HiP would allow the source population to benefit genetically from the growth in other reserves effectively reinstating a single large genetic population. There are still costs associated with translocation and not all of them are financial, but may also include short-term social disruption, reduction in breeding performance and death during capture and post 


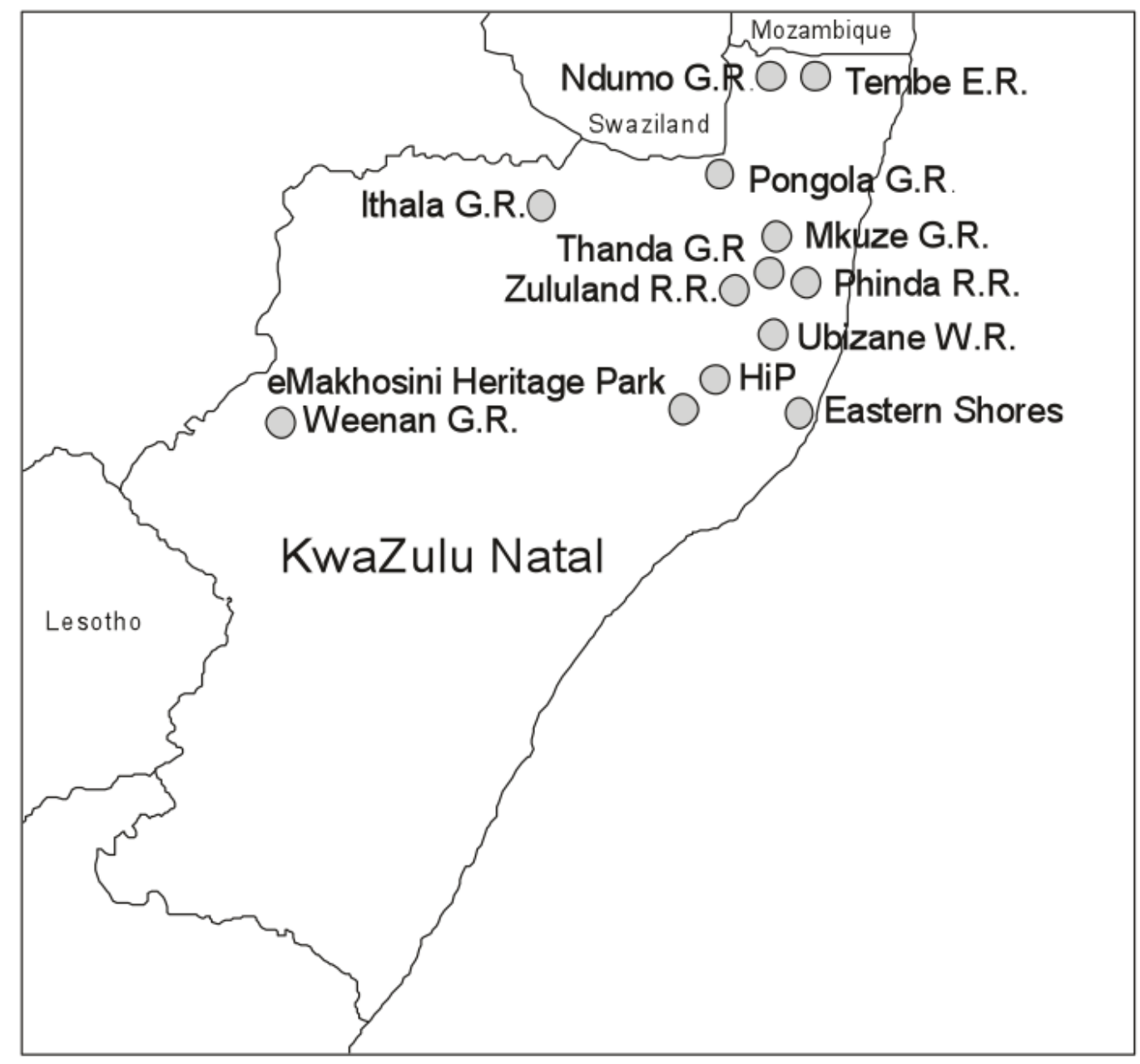

Figure 3.3: Black rhino game reserves in KZN. Ndumo Game Reserve, Tembe Elephant Reserve, Pongola Game Reserve, Ithala Game Reserve, Mkuze Game Reserve, Thanda Game Reserve, Phinda Resource Reserve, Zululand Rhino Reserve, Ubizane Wildlife Reserve, eMakhosini Heritage Park, HiP, Eastern Shores, Weenan Game Reserve

translocation (Hitchins 1984; Hall-Martin and Knight 1994; Adcock et al. 1998;

Linklater et al. 2011). However, benefits to KZN D. b. minor through of use of this type of adaptive management may outweigh associated costs of all types (Van Houtan et al. 2009).

\section{Genetic Replenishment}

Managers may also consider genetic replenishment by introducing native $D . b$. minor from Zimbabwe to KZN. The native D. b. minor population in Zimbabwe 
has higher mtDNA variation (six haplotypes; $n=11$ ) than the KZN

metapopulation (one mtDNA haplotype; $n=65$ ) (Anderson-Lederer et al. 2012) and has a higher microsatellite $H_{\mathrm{E}}$ and $H_{0}$ than KZN (Table 3.4). Although there may be concerns with how well the native Zimbabwe rhinos would thrive in KZN, the reverse scenario was successfully tested. In 1997, twenty-seven native KZN D. b. minor were translocated to Malilangwe, Zimbabwe where they were managed separately and not outbred with native Zimbabwe populations. The translocated KZN rhinos thrived with a growth rate of $8.3 \%$ per annum (du Toit 2001). The success of the translocated KZN D. b. minor to Zimbabwe may translate to native Zimbabwe D. b. minor being successfully translocated to KZN. However, before any native Zimbabwe $D . b$. minor are introduced to the KZN source populations, genetic supplementation experiments with a mixed population of KZN and native Zimbabwe $D . b$. minor should be cautiously and systematically established. F1 and F2 offspring of the mixed population could then be carefully monitored for signs of reduction in reproductive fitness (outbreeding depression), even though the likelihood of outbreeding depression in supplemented populations of the same species is low if they have the same karyotype, have been isolated for less than 500 years, and occupy similar environments (Frankham et al. 2011). An example of this type of mixed population is in Kruger National Park where 15 native Zimbabwe and 82 native KZN D. b. minor were introduced to the southern section of the nearly 2 million ha park from 1971 to 1988 (Hall-Martin and Castley 2001). The population would be ideal to study if stud books were kept and genetic samples collected during ear notching for identification events. 


\section{Possible Inbreeding Depression}

Experiments with mixed KZN and native Zimbabwe D. b. minor populations may also offer insight and solutions to possible inbreeding depression being expressed within the HiP population. Inbreeding depression and the way it impacts wild populations varies across taxa, populations and environments (Keller and Waller 2002). Garner et al. (2005) found that in populations that experienced one or more demographic threats (e.g. population declines, bottlenecks, reduction of population range) the level of genetic variation ( $>20 \%$ reduction in heterozygosity) was affected. Indeed, as the impact of genetic drift increases in small fragmented populations, genetic loss will reduce the range of possible adaptive responses in stressful environments (Bijlsma and Loeschcke 2012). Unfortunately, most stress resistance alleles have lower frequencies in populations and as genetic erosion takes place, those "rare" alleles have a higher probability of being lost (Bijlsma and Loeschcke 2012). While no outward signs of inbreeding depression have been identified, the average growth rate of the HiP population was only 3.4\% per annum (1999 - 2008) (Clinning et al. 2009). This is quite low when compared to the $6.75 \%$ per annum in KZP (Ferreira et al. 2011) and 8.3\% per annum (du Toit 2001) for the 27 translocated KZN $D . b$. minor to Zimbabwe mentioned earlier. The low growth rate and homozygote excess could be a result of genetic erosion or simply that Zimbabwe has a greater annual rain fall than South Africa which may increase the natural resources available to the black rhinos creating an environment more favourable to higher birth rates (Berkeley and Linklater 2010). 


\subsection{Conclusion}

It is important to detect losses in the level of genetic variation in $\mathrm{KZN}$, especially HiP where no translocations into the population take place. As poaching continues to threaten rhino populations across Asia and Africa, problems associated with managing small isolated populations grow with it. KZN black rhino managers have an opportunity to take necessary steps to curtail or stop inbreeding depression before it becomes detrimental to the metapopulation. With the KZN metapopulation exhibiting a single mtDNA haplotype and lower expected and observed heterozygosities than the native Zimbabwe D. b. minor (Table 3.3 \& 3.5) suggesting inbreeding, a more detailed management plan including data from this study may be required to prevent further loss of genetic variation, especially within $\mathrm{HiP}$. The natural migration process that black rhino were afforded before the 1600's may no longer be possible, but increasing reserve size, translocations of native $D$. $b$. minor from Zimbabwe to KZN or serial translocations from amongst the KZN metapopulation back into HiP may aid in maintaining current levels or increasing the overall genetic diversity of the source population that will translate to more diversity in the KZN metapopulation.

\subsection{Acknowledgements}

Thanks goes to Ezemvelo KZN Wildlife, specifically Drs. Dave Cooper and Dave Druce without whom this project would not have come to fruition, the Section Rangers, Game Capture Crew at Hluhluwe-iMfolozi Park, Geoff Clinning, and Dr. Jacques Flamand and Pam Sheriffs of WWF's Black Rhino Range Expansion Project for their support. Thanks also to South African National Parks, 
specifically Michael Knight, and Ministry of Environment and Tourism, Namibia, specifically Pierre du Preez and Rudi Loutit, for facilitating earlier sampling. Financial support from the U.S. Fish and Wildlife Service administered Rhinoceros and Tiger Conservation Act of 1994 (i.e., grant agreement number 96200-9-G268), including support of other projects facilitating earlier tissue and blood sampling (i.e. grant agreement numbers 98210-2-G363, 98210-4G920, and 98210-6-G102). 
Chapter III: Microsatellite DNA 


\section{CHAPTER FOUR}

Population Viability Analysis of Black Rhino (Diceros bicornis minor) in Hluhluwe-iMfolozi Game Park, KwaZulu-Natal, South Africa

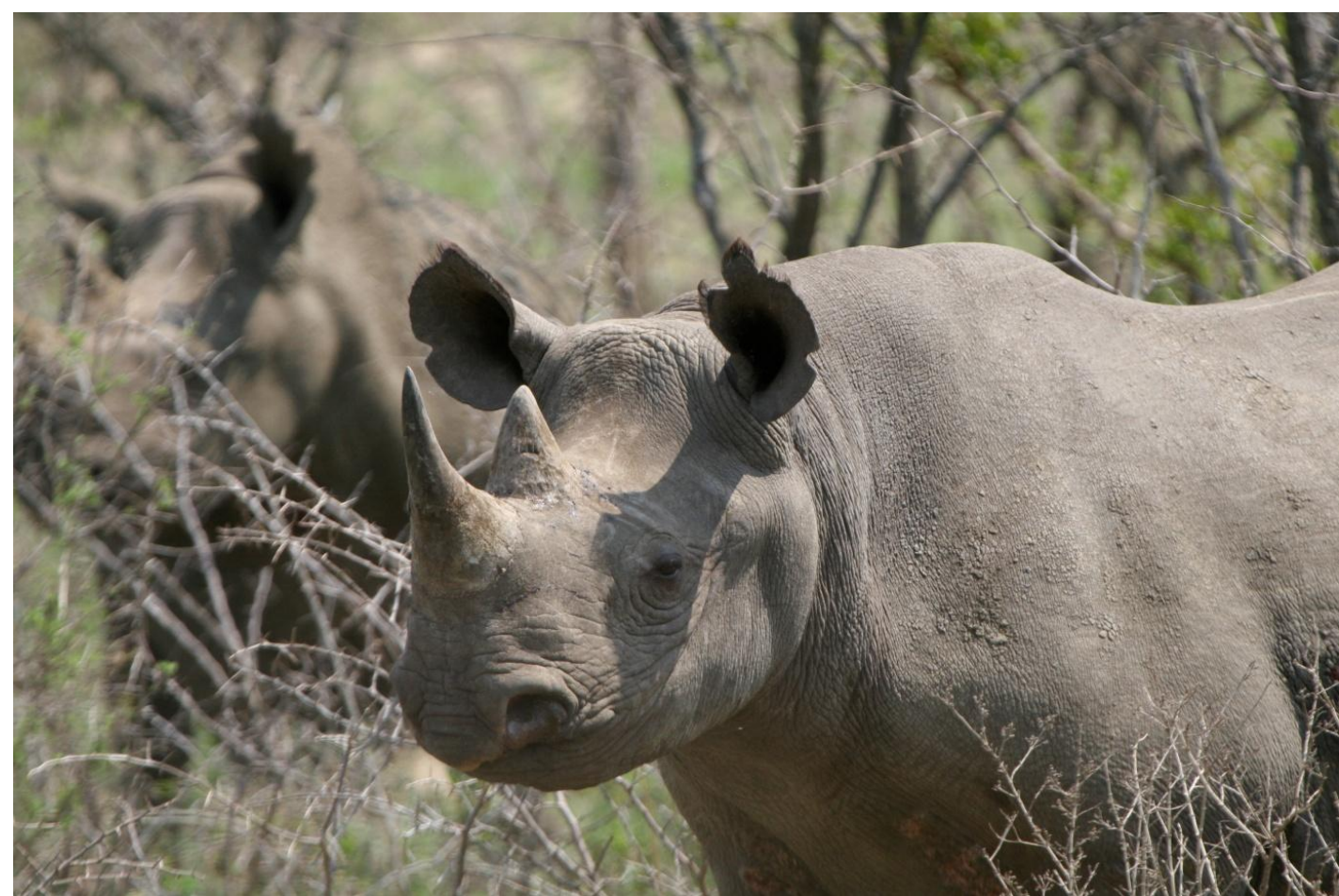

Photo by Rosalynn Anderson-Lederer 


\subsection{Abstract}

Once the loss of genetic diversity is identified in an endangered population, it is important for conservation managers to develop a plan to arrest further loss. Population viability analyses (PVA) are stochastic computer simulations used to predict the probability of future population persistence or extinction. Researchers use PVAs to visualize quantitative data to create informed management plans for endangered species. Hluhluwe-iMfolozi Game Park (HiP) in KwaZulu-Natal (KZN) is home to the largest remnant population of black rhino (Diceros bicornis minor) in South Africa and is a primary source for metapopulation expansion in South Africa. Unfortunately, HiP has significantly lower levels of mitochondrial and microsatellite DNA genetic variability than native D. b. minor populations in Zimbabwe. In this study, Vortex PVA was used to model population increases and supplementations into HiP with individuals from the KZN metapopulation and Zimbabwe. If current management remains unchanged, the PVA predicted a loss in the mean expected heterozygosity of $\sim 25 \%$ over $\sim 100$ black rhino generations (BRGs). Doubling the size of the modelled population decreased the rate of loss of the mean $H_{\mathrm{E}}$ by $\sim 10 \%$ over 100 BRGs. When supplementations of one female and one male black rhino from the KZN metapopulation were made every ten gestational years, the mean $H_{\mathrm{E}}$ of the population was maintained $(\sim 0.45)$ over $\sim 100 \mathrm{BRGs}$, but increased $\sim 30 \%$ when supplemented with one female and one male from Zimbabwe. PVA results indicate that artificial game park expansion through supplementation is effective and does not require a large number of individuals or frequent translocation. Based on these results, HiP managers should consider 
incorporating a supplementation regime into current management plans to prevent further loss of genetic diversity within this valuable source population.

\subsection{Introduction}

The task of restoring populations of vulnerable and endangered species while maintaining genetic diversity is challenging because remnant populations are inevitably small. When a species which had an historically large and widespread population is fragmented into smaller isolated populations, genetic drift can quickly eliminate genetic variability (Lacy 1987b). In response to the problem of small populations the idea of a minimum viable population (MVP) size was introduced. Unfortunately, population sizes required to prevent variability loss tend to be significantly larger than the targets set by conservation managers and organizations (Traill et al. 2010). Nevertheless, increasing population sizes to match the MVP is not always possible due to habitat size limits. Adding conspecific individuals (supplementations) to the population (IUCN 1987), however, can be carried out via serial translocations. Supplementations via serial translocations among small populations would create an artificial metapopulation that would make the effective population size $\left(\mathrm{N}_{\mathrm{e}}\right)$ large enough to match the MVP size. The $\mathrm{N}_{\mathrm{e}}$ could in turn prevent the loss of genetic diversity in small, fragmented populations (Waite et al. 2005).

Similar to MVPs, population viability analyses (PVA) use a stochastic computer simulation to forecast the likelihood of future population persistence or extinction using species specific life-history data (vital rates: e.g. age, reproductive rates, mortality, breeding system) (Boyce 1992). Conservation managers typically use the outcomes of PVAs to visualize quantitative data (e.g. 
demographic, ecological, and genetic) to establish policy priorities and develop realistic targets (e.g. fiscal, technical, personnel use) (Possingham et al. 1993; Lindenmayer et al. 1993).

The species life-history data used to create models can be difficult to obtain from small populations of some species, which means PVA models have an inherent uncertainty in their results (Shaffer 1990; Lindenmayer et al. 1993). That being said, retrospective PVAs performed on birds (e.g. black-capped chickadee (Parus atricapillus), mammals (e.g. Cape hunting dog (Cynomys ludovicianus), fish (brook trout (Salvelinus fontinalis) and reptiles (sage-brush lizard (Sceloporus graciosus)) were proven to be a reliable and effective tool for managing endangered species (Brook et al. 2000). Several PVA models for wild and captive black rhino populations have also been reported.

Analyses of founder members for captive populations, demographic stability, and loss of genetic variability in reserves in Kenya and Tanzania as well as the viability of captive black rhinos have been investigated using VoRTEX PVA (Lacy 1987a; Foose 1987; Moehlman et al. 1996). In addition, conservation strategies including carrying capacity, population structure and densitydependence (Swart et al. 1990; Adcock 2001; Cromsigt et al. 2002; Dunn et al. 2007) have been investigated using a range of MVP and PVA modelling techniques. With a black rhino generation time of c. 14 years (Brooks and Adcock 1997) it is too early to corroborate most of the simulated results with real populations that have been modelled. Nonetheless, population estimates of black rhino in the Ngorongoro Crater, Tanzania in 2006 (Mills et al. 2006) were comparable with predicted PVA estimates from simulations made in 1996 (Moehlman et al. 1996) confirming the predicted outcome of the black rhino 
population. In addition, the retrospective study by Cromsigt et al. (2002) examining structure and density-dependence models demonstrated that out of five deterministic models tested, Fowler's translocation model (Fowler 1981) best fitted the Hluhluwe-iMfolozi (HiP) and Mkuze Game Reserve (MGR) population and translocation censuses (HiP 1990 - 1998; MGR 1989 - 1998), again showing that computer models based on black rhino data can produce realistic population outcomes.

The HiP D. b. minor population is important in the black rhino recovery programme because it is the largest endemic and remnant population of $D . b$. minor in South Africa. Recent findings of significantly lower microsatellite DNA variation in KwaZulu-Natal (KZN) D. b. minor than native Zimbabwe D. b. minor (KZN: $H_{\mathrm{E}}=0.47, H_{0}=0.49$; Zimbabwe: $H_{\mathrm{E}}=0.65, H_{0}=0.72$; Chapter 3 ) and low mtDNA variation (KZN: one haplotype, $n=65$; Zimbabwe: six haplotypes, $n=11$ Anderson-Lederer et al. 2012) strongly suggest a loss of genetic diversity within $\mathrm{HiP}$ and the KZN metapopulation requiring alterations to current management strategies to preserve genetic diversity.

There are several possible management responses that could be taken to reduce the loss of genetic variation. One such strategy is to expand the size of the game reserve. This has been proposed for HiP by connecting it via corridors with other neighbouring game parks including Zululand Game Reserve, Mkuze Game Reserve, Phinda Resource Reserve and Greater St. Lucia Wetland Park. This strategy however, has limits including the cost of purchasing land, increased management expenses (e.g. additional monitoring, fencing) and the logistics of creating corridors. In addition, the land must fulfill strict black rhino nutritional requirements for optimal breeding at pre-set carrying capacities 
(CC) enforced by state agencies (Hall-Martin and Castley 2001; Emslie et al. 2009). It is unclear, though how much area would be required to slow the rate of loss in the level of genetic variation for the population.

Another strategy is serial translocation that exchanges individuals between small populations allowing each population to benefit from immigration. Limitations for this strategy involve costs associated with translocations. Expenses include, but are not limited to the capture and holding of animals (e.g. trucks, helicopters, fuel, transportation crates, darting medications), as well as legal and biological considerations (e.g. age and sex of animals being moved) (Emslie et al. 2009). If the translocation takes place across international borders, the process can be even more complicated by government involvement (Emslie et al. 2009). It is uncertain however, how many individuals would need to be moved between populations to slow the rate of loss in the level of genetic variation for the population.

PVA modelling can be used to determine which management scenario might be most effective. When choosing a PVA programme for modelling possible management scenarios, Lindenmayer et al. (1995) suggests selection criteria be based on (1) the primary objectives of the study and (2) the strengths, limitations and assumptions of the programme and how these correspond to the traits, life-history parameters, quality and quantity of available data for the species being modelled. The criteria selection is important because not all PVAs calculate outcomes in the same way and what may be appropriate for one type of population may not be appropriate for others (e.g. closed population versus metapopulation, long-lived species versus shortlived). VORTEX has been rigorously examined in peer-reviewed studies and 
population forecasts have been shown to be accurate when sufficient and accurate species specific life-history data are available (Brook et al. 1997; Brook et al. 1999; Brook et al. 2000; Coulson et al. 2001).

The objective of this study was to use a PVA to examine a set of management strategies and determine the most effective management plan for preventing the loss of genetic variation within the HiP population. Included in the analyses are scenarios that increase the population size and supplementations made with individuals representing the KZN metapopulation and individuals representing the native Zimbabwe D. b. minor population. The results of the models are used to make management recommendations for the HiP population.

\subsection{Methods}

Available life-history data of the HiP population of $D$. $b$. minor were incorporated into the PVA model. When specific data for HiP black rhino were unavailable, information from other populations of black rhino were used from published literature and unpublished reports. Life-history data and model parameters were set as follows (Table 4.1):

\subsubsection{Species Description}

\section{Number of iterations}

The model used a random number generator so that none of the repeated simulations would be the same (Miller and Lacy 2005); therefore, 100 iterations is usually adequate to uncover tendencies (Lacy 1993); however, between 500 
Table 4.1: Vortex scenario parameters

\begin{tabular}{|c|c|c|}
\hline Vortex Parameters & Value & Source \\
\hline \multicolumn{3}{|l|}{ Species Description } \\
\hline Iterations & 1000 & This study \\
\hline No. of years & 1000 'gestational' (1342 actual) & This study \\
\hline Duration of each "year" & $\begin{array}{l}490 \text { days (adjusted to accommodate } \\
\text { length of black rhino pregnancy) }\end{array}$ & This study \\
\hline Extinction def: & one sex remains & This study \\
\hline No. of Populations & 1 & This study \\
\hline Inbreeding depression & Yes \& No & This study \\
\hline \multicolumn{3}{|l|}{ Reproductive System } \\
\hline Polygynous & Polygynous & Garnier et al. 2001 \\
\hline Age of First offspring for females & $6^{+}$(gestational years) & Owen-Smith 1988 \\
\hline Age of First offspring for males & $7^{+}$(gestational years) & Lent \& Fike 2003 \\
\hline \multirow[t]{2}{*}{ Maximum Age of Reproduction } & $27^{+}$(gestational years) & Shenkle \& Shenkle 1969 \\
\hline & & Owen-Smith 1988 \\
\hline Maximum no. of broods per year & 1 & Owen-Smith 1988 \\
\hline Maximum no. of progeny per brood & 1 & Owen-Smith 1988 \\
\hline Sex ratio at birth in $\%$ males & 53 & Berkley \& Linklater 2010 \\
\hline Density Dependent Reproduction & $=\left(32-\left((32-28) *\left((\mathbf{N} / \mathrm{K})^{\wedge} 8\right)\right)\right) *(\mathrm{~N} / 0+\mathrm{N})^{*}$ & This study \\
\hline \multicolumn{3}{|l|}{ Reproductive Rates } \\
\hline$\%$ Adult females breeding & $32 \%$ & Clinning 2009 \\
\hline environmental variation in \% breeding & 3 & Clinning 2009 \\
\hline \multicolumn{3}{|l|}{ Mortality Rates Female in \% } \\
\hline from age $0-1$ & $15 \%{ }^{+}$(adjusted from $11 \%$ )* & Adcock and Emslie 2003 \\
\hline from age $1-4$ & $4 \%^{+}$(adjusted from $\left.3 \%\right) *$ & Adcock and Emslie 2003 \\
\hline from age $4-6$ & $8 \%{ }^{+}$(adjusted from $5.7 \%$ ) $*$ & Owen-Smith 1988 \\
\hline Mortality from age 6 and above & $5 \%{ }^{+}$(adjusted from $3.5 \%$ )* & Owen-Smith 1988 \\
\hline \multicolumn{3}{|l|}{ Mortality rate Male \% } \\
\hline Mortality from age $0-1$ & $15 \%^{\dagger}$ (adjusted from $11 \%$ )* & Adcock and Emslie 2003 \\
\hline from age $1-4$ & $4 \%{ }^{\dagger}$ (adjusted from $3 \%$ )* & Adcock and Emslie 2003 \\
\hline from age $4-6$ & $8 \%{ }^{\dagger}$ (adjusted from $5.7 \%$ )* & Owen-Smith 1988 \\
\hline Mortality from age 6 and above & $10 \%{ }^{+}$(adjusted from $\left.7.3 \%\right) *$ & Owen-Smith 1988 \\
\hline \multicolumn{3}{|l|}{ Mate Monopolization } \\
\hline$\%$ males breeding in pool & 100 & \\
\hline$\%$ males successfully siring offspring & 37.1 (Calculated by Vortex) & \\
\hline Mean \# of mates/successful sire & 1.2 (Calculated by Vortex) & \\
\hline \multicolumn{3}{|l|}{ Initial population size } \\
\hline Stable Age Distribution & 300 & Fanayo et al. 2005 \\
\hline \multicolumn{3}{|l|}{ Carrying Capacity } \\
\hline Carrying capacity (K) & $430 \& 860^{\ddagger}$ & Emslie 2009 \\
\hline $\mathrm{SD}$ in $\mathrm{K}$ due to environmental variation & 43,86 & Emslie 2009 \\
\hline \multicolumn{3}{|l|}{ Harvest } \\
\hline Population Harvested? & Yes & Emslie 2009 \\
\hline First year of harvest? & 1 & Emslie 2009 \\
\hline Last year of harvest? & 1000 & Emslie 2009 \\
\hline Interval between harvests & 1 & Emslie 2009 \\
\hline Optional Criterion for harvest & $=(W+X) \geq(K / 2)$ & \\
\hline Supplementation & $1 F, 1 M ; 2 F, 2 M$ & This study \\
\hline first year of supplementation & 1 & This study \\
\hline last year of supplementation & Varied & This study \\
\hline interval between supplementations & $1,2,5, \& 10$ years (gestational) & This study \\
\hline
\end{tabular}

Number of microsatellite loci examined 10 from KZN pop. \& 10 from Zim. pop This study, Garnier et al. 2001 + Variation in age and mortality greatly affect PVA outcomes, younger ages and and older mortality rates increase reproductive output over an animal's life; Based on density dependent variables, see text for details; * Percentages are adjusted to reflect a longer year of 490 days; $\neq$ Carrying capacities based on HiP CC, see text for details 
and 1000 iterations are encouraged to provide more rigorous results (Miller and Lacy 2005). One thousand iterations were chosen for the models run in this study.

\section{Duration of a year}

The length of a year was adjusted from 365 days (default) to 490 days in order to satisfy 'maximum number of broods per year' in the "Reproductive System" section of the parameter settings (Lacy, R. pers. comm.). Entries made for 'maximum number of broods per year' must be a whole number. The gestation period for a black rhino is 460 days (15.33 months) (Linklater 2007). In order to enter an integer (i.e. 1) instead of a fraction (i.e. 0.8), a 'year' was adjusted to reflect 490 days to accommodate one brood per year, plus an additional 30 days, the minimum time required to become pregnant again. This was done to avoid over-estimating the number of births in the simulations. The adjusted year $(460+30$ days $)$ is referred to as the 'gestational year'.

\section{Number of years}

Simulations were run for 1000 gestational years to see how genetic variation changed over a lengthy time period. One thousand gestational years translates to 1342 calendar years. According to the Conservation Plan for the Black Rhinoceros in South Africa (Brooks and Adcock 1997) one black rhino generation (BRG) is c. 14 years. One thousand gestational years enabled visualization of approximately 96 BRGs.

\section{Inbreeding depression}

Previous black rhino microsatellite DNA studies concluded that the level of genetic variation in D. b. minor was not low enough to be of concern (Harley et 
al. 2005; Karsten et al. 2011). However, since KZN D. b. minor has only one mtDNA haplotype (Anderson-Lederer et al. 2012) and homozygote excess was observed in the 10 microsatellite DNA loci examined (Chapter Three), scenarios with and without inbreeding were run to compare how inbreeding depression influences the outcome for the simulated population. In scenarios with inbreeding depression, a default value of lethal equivalents (3.14) was selected; the default is based on Ralls et al. (1988) survey of 40 mammal populations (Miller and Lacy 2005). When modelling inbreeding depression, the model reduced the survival of offspring only in the first year, which caused the results of inbreeding depression to be conservative (Miller and Lacy 2005).

\section{Catastrophes}

No catastrophes (e.g. drought and disease) were modelled in these scenarios. Environmental variation is reflected in other parameters, and this project is focused on establishing baseline genetic results for increasing population sizes and supplementation, not addressing how catastrophes affect the population.

\subsubsection{Reproductive System}

\section{Age of first offspring for females}

Female black rhino first give birth between ages 6.5 and 8.5 (Owen-Smith 1988). Using the average of 7.5, age was adjusted to 6 years based on a gestational year ( 7.5 years $x 365.25$ days $/ 490$ days $=5.59$ years, $\sim=6$ ). This number appears high because black rhino females become sexually mature as early as 3.5 - 4 years old (Schenkel and Schenkel-Hulliger 1969), but the model population is assumed to be near carrying capacity (CC). Large mammal density-dependence is expected to be weak except near CC, where it is reflected 
in low reproductive rates especially in younger females which exhibit delayed first reproduction (Fowler 1981). An increase in the age of first offspring will mean a reduced reproductive output over the life of the female, but the mean age was chosen to reflect conservative outcomes for the simulated population.

Age of first offspring for males

Males successfully reproduce at approximately 9 years old (Owen-Smith 1988; Bertschinger 1994; Lent and Fike 2003). Age was adjusted from 9 years to 7 years based on a gestational year ( 9 years x 365.25 days $/ 490$ days $=6.70$ years, $\sim=7$ ).

\section{Maximum age of reproduction, number of progeny per year}

Black rhino females have one offspring per pregnancy. The maximum age of reproduction is approximately 37 years old (Schenkel and Schenkel-Hulliger 1969; Owen-Smith 1988). Age was adjusted to 28 based on a gestational year (37 years $\mathrm{x} 365.25$ days $/ 490$ days $=27.58, \sim=28$ ).

Sex ratio at birth - in \% male

The sex ratio of black rhino across combined age groups averages to approximately 1:1 (Hillman-Smith and Groves 1994); however, the proportion of males detected soon after birth is slightly higher (53\%) (Emslie et al. 2009; Berkeley and Linklater 2010).

\section{Density-dependent reproduction}

Large-bodied species show a life-history strategy that includes slow growth rates with fitness components (e.g. infant mortality, reproductive rates) that are affected as populations near CC (Eberhardt 1977; Fowler 1981; Gaillard et al. 
2000). Density-dependence first affects the youngest members of a population with infant and juvenile mortality highest when populations approach CC (Gaillard et al. 2000). Fecundity of young females is next to be affected, followed by adult females and then adult (male and female) survival (Gaillard et al. 2000). The influence of density-dependence has been observed in several large ungulate species, including wildebeest (Mduma et al. 1999), caribou (Messier et al. 1988; Tews et al. 2007), wild reindeer (Skogland 1985), roe deer (Kjellander et al. 2004) and northern fur seals (Fowler 1990).

The function used for modelling was Density-Dependent Reproduction = $\left.\left(32-\left((32-28) *(\mathrm{~N} / \mathrm{K})^{\wedge} 8\right)\right)\right)^{*}(\mathrm{~N} / 0+\mathrm{N})$, where $\mathrm{N}=$ population size, $\mathrm{K}=$ carrying capacity and $\mathrm{P}=$ population identifier, based on the following:

- \% Breeding at Low Density, P (0): 32 (Clinning et al. 2009)

- \% Breeding at Carrying Capacity (Maximum Age), P (K): 28 (Miller and Lacy 2005)

- Allee Parameter A: 0 Vortex manual: (Miller and Lacy 2005)

- Steepness Parameter B: 8 Vortex manual: (Miller and Lacy 2005)

\subsubsection{Reproductive Rates}

\section{$\%$ Adult Females Breeding}

The percent of adult females breeding is set automatically based on the densitydependence variables entered in the model.

\subsubsection{Mortality Rates}

Mortality rates were based on Owen-Smith's (1988) observations from HiP. Any age groups that were not documented by Owen-Smith were supplemented with mortality rates for black rhino from South Africa and Namibia from Adcock and 
Emslie (2003). All mortality rates were adjusted to accommodate the gestational year (Table 4.1).

\subsubsection{Initial Population Size}

Between 1930 - 2009, black rhino numbers at HiP ranged between 130 to 400 individuals (Emslie 2011; Fanayo et al. 2005), although Clinning et al. (2009) determined that past census numbers were over-estimated, in some years by as much as $48 \%$. Since it is impossible to know the exact number of individuals in a wild population, 300 was entered as the initial population size and the default 'stable age distribution' was chosen.

\subsubsection{Carrying Capacity}

Management cannot increase a reserve's CC unless the size of the reserve is increased through land acquisition. However, an understanding of how a population's size is affected by its CC is important in order to decrease effects of density-dependence when drafting black rhino management plans (Adcock 2001). Owen-Smith (2001) defines CC as the number of individuals a population can sustain (relying on resources in the area) that remains constant due to births cancelling out deaths. Brooks and Adcock (1997) estimate that the CC for HiP black rhino is 430 individuals. Their estimates are based on frost ratings, vegetation and approximate annual rainfall each year. Carrying capacity for the simulated population was therefore set at 430 for baseline scenarios. If the simulated population were to increase in size (e.g. HiP merging with neighbouring game reserves), it may be able to double its CC $(\sim 860)$; therefore, an 860 CC was also used to visualize how increasing the size of the simulated population would affect the population from a genetic perspective. 


\subsubsection{Harvest (Capturing individuals to relocate to other reserves)}

\section{Harvest criteria}

Harvests occurred every gestational year (490 days).

\section{Optional criteria for harvest}

Parameters were set to only harvest if the total population size was $50 \%$ or more of the CC (Emslie et al. 2009). The function used for this was Optional Criteria $=(\mathrm{W}+\mathrm{X}) \geq(\mathrm{K} / 2)$, where $\mathrm{X}$ is females in the population, $\mathrm{W}$ is males in the population and $\mathrm{K}$ is the carrying capacity (Lacy, R. pers. comm.). If during any year of a harvest the total population was less than $50 \%$ of the carrying capacity, no harvest took place (Miller and Lacy 2005).

\section{Number of female and male of each age to be harvested}

As suggested by translocation studies, the model harvested only adult females and males (Linklater et al. 2011; Linklater et al. 2012) because young translocated black rhino experience higher mortality rates than their adult counterparts and young females have lower fecundity rates after translocations. The SADC recommends harvest rates of 5\% - 8\% for black rhino (Emslie 2001). The percentage of black rhino removed from the modelled population was set at $4 \%$ of the total population per gestational year (which translates to $5.4 \%$ for a calendar year). This percentage was chosen because it was conservative and also because it is the harvesting goal of HiP (Clinning et al. 2009). If harvesting took place during a particular year (population size was more than $50 \%$ of the carrying capacity), the $4 \%$ was split between females and males (e.g. $2 \%$ of the total population was removed from the adult females ( $\geq 6$ years old) and $2 \%$ of the total population was removed from the adult males ( $\geq 7$ years old). The 
functions used for this were Females Harvested $=\mathrm{X}^{*} \%$ and Males Harvested $=$ $\mathrm{W} * \%$, where $\mathrm{X}$ was females, $\mathrm{W}$ was males, and $\%$ was half of the percent harvested that year.

\subsubsection{Supplementation}

Frequency of supplementations

Supplementations occurred every year (E1Y), two years (E2Y), 5 years (E5Y) and ten years (E10Y).

Number of females and males supplemented

Supplementations were made with one female and one male $(1 \mathrm{~F}, 1 \mathrm{M})$ and two females and two males $(2 \mathrm{~F}, 2 \mathrm{M})$ at each of the time periods simulated.

\subsubsection{Genetic Management}

Data from 10 microsatellite DNA loci for the KZN metapopulation (Chapter 3) with an $H_{\mathrm{E}}$ of 0.47 were imported to the PVA to reflect the current level of genetic diversity for the modelled population (Table 4.2).

Supplementations were made with two different types of supplemental individuals, those representing the KZN metapopulation ( $H_{\mathrm{E}}$ of 0.47 ; Table 4.2) and those representing the native Zimbabwe $D$. $b$. minor population $\left(H_{\mathrm{E}}\right.$ of 0.62 ; Table 4.3). 
Table 4.2: Allele frequencies for the 10 microsatellite DNA loci examined in Chapter 3 for HiP $D . b$.

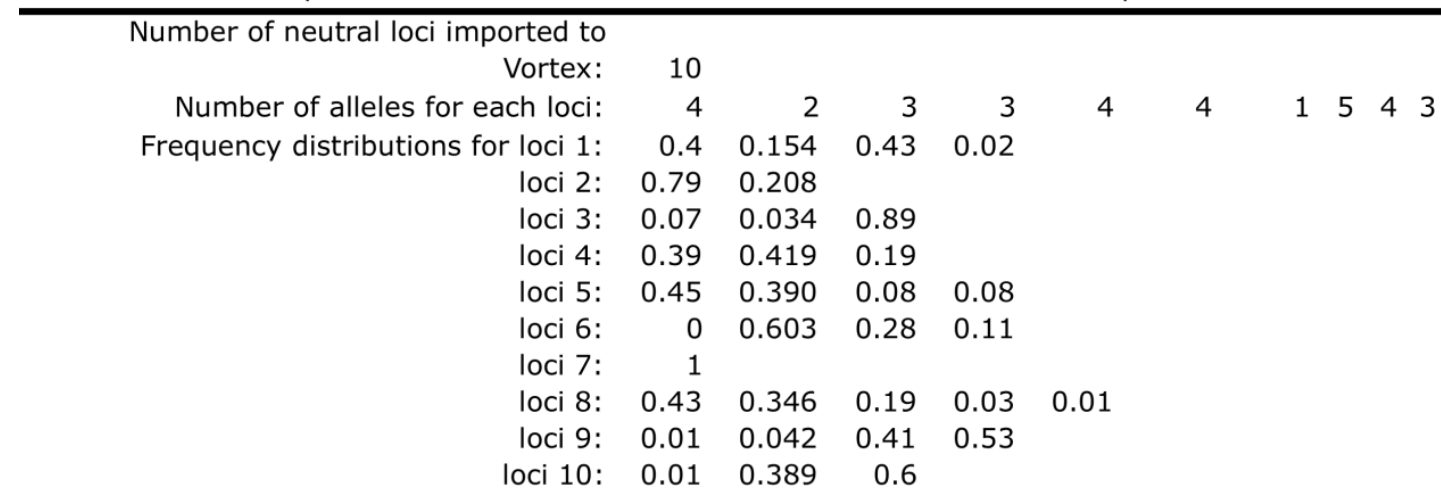

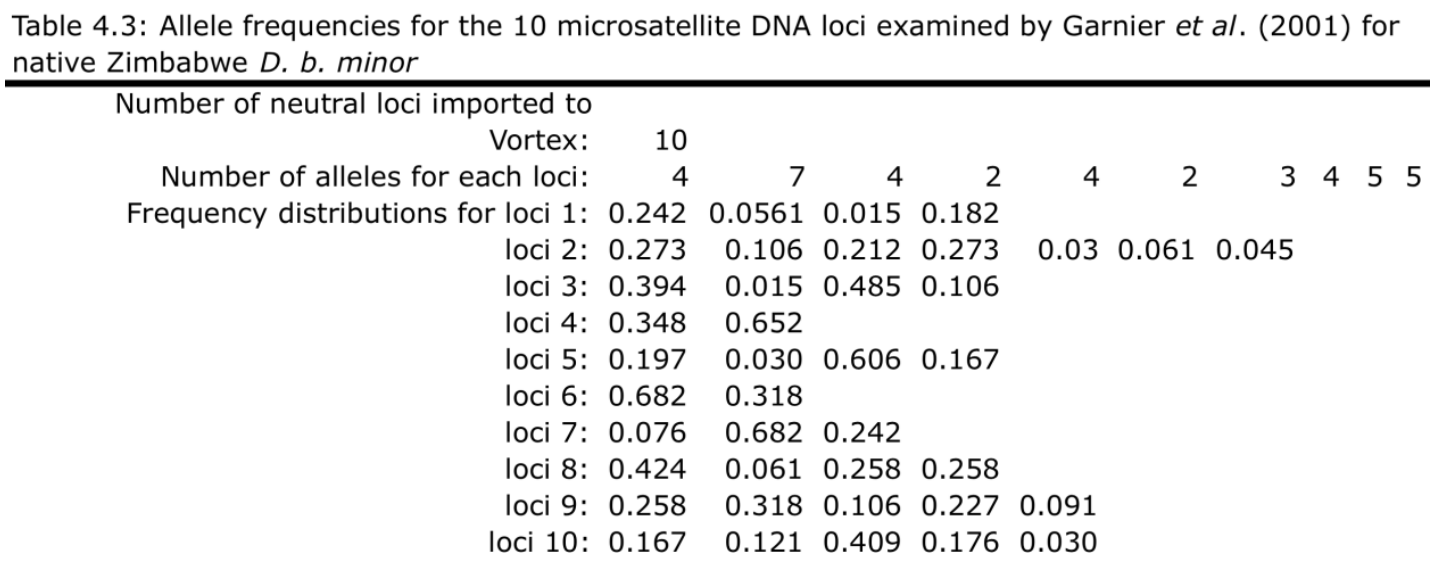

\subsection{Results}

\subsubsection{Levels of Genetic Variation with No Supplementation}

When current levels of genetic variation observed at 10 microsatellite DNA loci in the KZN metapopulation ( $H_{\mathrm{E}}$ of 0.47$)$ were assigned to the modelled population (CC 430) with no inbreeding depression and no supplementation, the population decreased in size from 300 where it then stabilised to approximately 259 individuals (Figure 4.1). In the model population with no supplementation and a CC of 430, mean expected heterozygosity $\left(H_{\mathrm{E}}\right)$ averaged over 10 microsatellite loci decreased by 3\%,11\% and 23\% over 19, 48 and 96 
BRGs respectively (200, 500 and 1000 gestational years) (Table 4.4). When the model population's CC was doubled to 860 , to simulate the outcome of increasing the size of the reserve (e.g. merging with other reserves), genetic variation decreased over time, but at a slower rate than the 430 CC (Table 4.5). The mean $H_{\mathrm{E}}$ averaged over 10 microsatellite loci was maintained for approximately 24 BRGs (250 gestational years), but then began to decline. There was a decrease in the mean $H_{\mathrm{E}}$ of $4 \%$ and $11 \%$ over 48 and 96 BRGs respectively (500 and 1000 gestational years respectively) (Table 4.5).

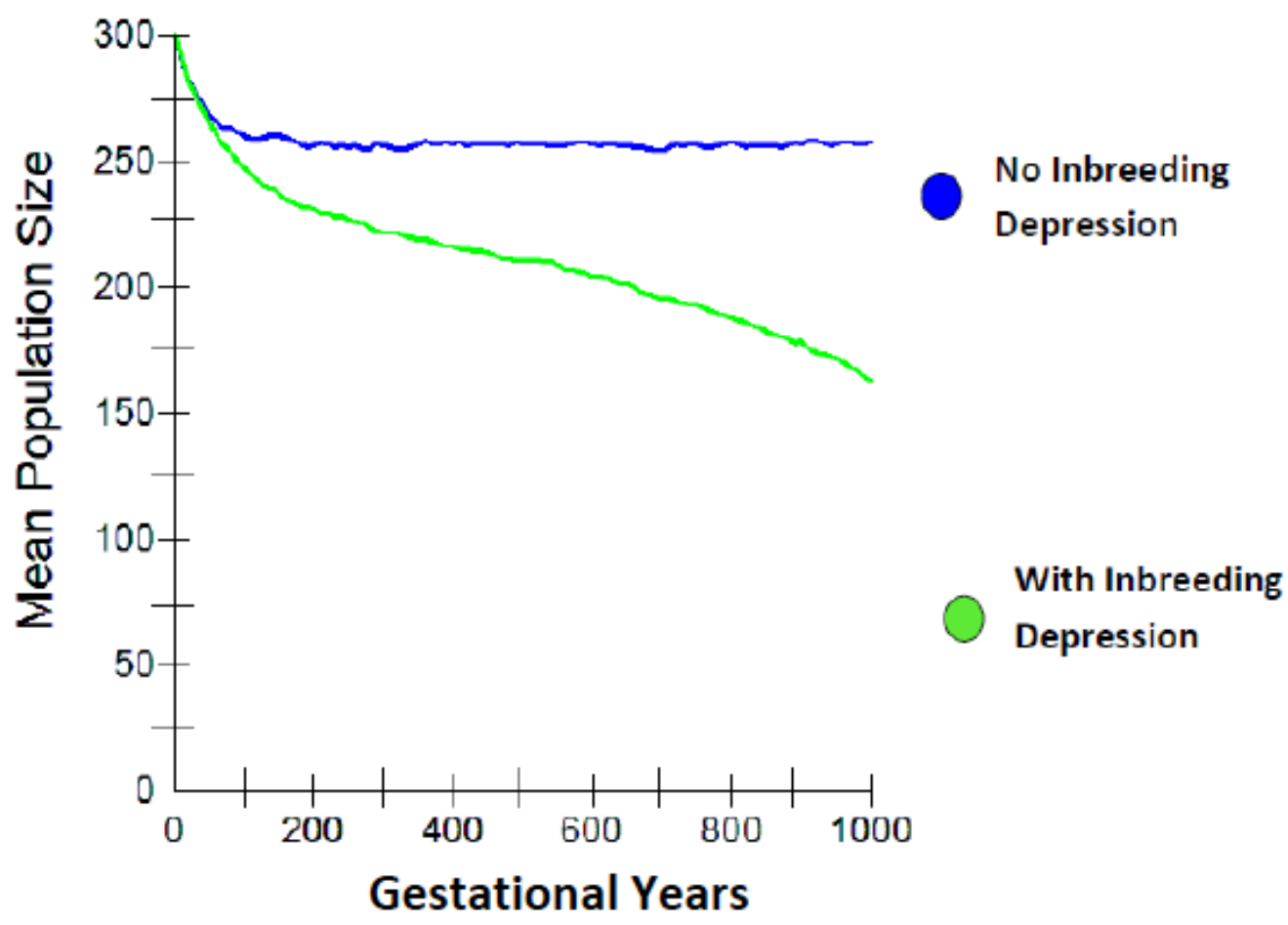

Figure 4.1: The mean population sizes for a modelled population with a CC of 430, with and without inbreeding depression, and a 4\% harvesting rate per gestational year (translates to $5.4 \%$ in a calendar year). 
Table 4.4: Expected and observed heterozygosities $\left(H_{\mathrm{E}}\right.$ and $\left.H_{\mathrm{O}}\right)$ with standard error for HiP $D . b$. minor; with and without inbreeding depression at 430 carrying capacity for years 50, 100, 150, 200, 250, 500, $700 \& 1000(5,10,14,19,24,48,67$, and 96 black rhino generations respectively); with mean population sizes and standard error. The $4 \%$ harvest is for a gestational year, which translates to $5.4 \%$ in a calendar year.

\begin{tabular}{|c|c|c|c|c|c|c|}
\hline \multirow[t]{2}{*}{ Year } & \multirow[t]{2}{*}{$\sim$ Pop Size } & \multicolumn{2}{|c|}{$\begin{array}{l}\text { No Inbreeding Depression, } \\
\text { No Genetic Supplementation }\end{array}$} & \multirow[t]{2}{*}{ Pop Size } & \multicolumn{2}{|c|}{$\begin{array}{l}\text { Inbreeding Depression, } \\
\text { No Genetic Supplementation }\end{array}$} \\
\hline & & $H_{\mathrm{E}}$ & $H_{\circ}$ & & $\underline{H_{\mathrm{E}}}$ & $H_{0}$ \\
\hline 50 & $267 \pm 1.25$ & $0.459 \pm 0.000$ & $0.462 \pm 0.000$ & $263 \pm 1.21$ & $0.459 \pm 0.000$ & $0.462 \pm 0.000$ \\
\hline 100 & $260 \pm 1.19$ & $0.451 \pm 0.001$ & $0.453 \pm 0.001$ & $247 \pm 1.09$ & $0.453 \pm 0.001$ & $0.455 \pm 0.001$ \\
\hline 150 & $257 \pm 1.16$ & $0.446 \pm 0.001$ & $0.448 \pm 0.001$ & $238 \pm 1.01$ & $0.447 \pm 0.001$ & $0.450 \pm 0.001$ \\
\hline 200 & $258 \pm 1.21$ & $0.438 \pm 0.001$ & $0.441 \pm 0.001$ & $230 \pm 0.91$ & $0.440 \pm 0.001$ & $0.444 \pm 0.001$ \\
\hline 250 & $256 \pm 1.20$ & $0.432 \pm 0.001$ & $0.435 \pm 0.001$ & $226 \pm 0.90$ & $0.432 \pm 0.001$ & $0.436 \pm 0.001$ \\
\hline 500 & $259 \pm 1.23$ & $0.402 \pm 0.001$ & $0.404 \pm 0.001$ & $211 \pm 0.77$ & $0.397 \pm 0.001$ & $0.400 \pm 0.001$ \\
\hline 700 & $256 \pm 1.18$ & $0.379 \pm 0.001$ & $0.381 \pm 0.001$ & $199 \pm 0.82$ & $0.370 \pm 0.001$ & $0.373 \pm 0.001$ \\
\hline 1000 & $257 \pm 1.18$ & $0.347 \pm 0.002$ & $0.349 \pm 0.002$ & $165 \pm 1.39$ & $0.339 \pm 0.002$ & $0.333 \pm 0.002$ \\
\hline
\end{tabular}

Table 4.5: Expected and observed heterozygosities $\left(H_{\mathrm{E}}\right.$ and $\left.H_{\mathrm{O}}\right)$ with standard error for HiP $D . b$. minor; with and without inbreeding depression at 860 carrying capacity for years 50, 100, 150, $200,250,500,700 \& 1000(5,10,14,19,24,48,67$, and 96 black rhino generations respectively); with mean population sizes and standard error. The $4 \%$ harvest is for a gestational year, which translates to $5.4 \%$ in a calendar year.

\begin{tabular}{|c|c|c|c|c|c|c|}
\hline \multicolumn{7}{|c|}{860 carrying capacity, $4 \%$ Harvest } \\
\hline \multirow[t]{2}{*}{ Year } & \multirow[t]{2}{*}{$\sim$ Pop Size } & \multicolumn{2}{|c|}{$\begin{array}{l}\text { No Inbreeding Depression, } \\
\text { No Genetic Supplementation }\end{array}$} & \multirow[t]{2}{*}{$\sim$ Pop Size } & \multicolumn{2}{|c|}{$\begin{array}{l}\text { Inbreeding Depression, } \\
\text { No Genetic Supplementation }\end{array}$} \\
\hline & & $H_{\mathrm{E}}$ & $H_{0}$ & & $H_{\mathrm{E}}$ & $H_{0}$ \\
\hline 50 & 473 & $\overline{0.461}$ & 0.46 & 4 & $0.461 \pm 0.000$ & 0.000 \\
\hline 100 & $503 \pm 1.85$ & $0.458 \pm 0.000$ & $0.459 \pm 0.000$ & $491 \pm 1.85$ & $0.458 \pm 0.000$ & 0.000 \\
\hline 150 & $514 \pm 2.09$ & $0.454 \pm$ & 0.4 & 1.85 & 0. & 000 \\
\hline 200 & $517 \pm 2.14$ & $0.451 \pm$ & $0.452 \pm$ & $478 \pm 1.65$ & .001 & 0.001 \\
\hline 250 & $519 \pm 2.09$ & $0.448 \pm 0.001$ & $0.449 \pm 0.001$ & $470 \pm 1.59$ & $0.447 \pm 0.001$ & $0.449 \pm 0.001$ \\
\hline 500 & $516 \pm 2.04$ & $0.432 \pm 0.001$ & $0.433 \pm 0.001$ & $452 \pm 1.33$ & $0.430 \pm 0.001$ & $0.432 \pm 0.001$ \\
\hline 700 & $516 \pm 2.04$ & $0.419 \pm 0.001$ & $0.421 \pm 0.001$ & $442 \pm 1.25$ & $0.416 \pm 0.001$ & $0.418 \pm 0.001$ \\
\hline 1000 & $517 \pm 2.11$ & $0.402 \pm 0.001$ & $0.403 \pm 0.001$ & $425 \pm 1.28$ & $0.395 \pm 0.001$ & $0.397 \pm 0.001$ \\
\hline
\end{tabular}

\subsubsection{Level of Variation with Supplementations}

Supplementations of one pair (1F, 1M) of KZN D. b. minor made to the simulated population (CC 430) approximately every BRG (10 gestational years, 13.4

calendar years) showed a negligible increase in mean population size (Figure

4.2). Supplementations made with one pair (1F, 1M) of KZN rhino every one and 
two gestational years maintained the mean $H_{\mathrm{E}}(\sim 0.45)$ through 96 BRGs $(1000$ gestational years) (Table 4.6; Figures 4.3 A-B). When supplementations of the pair were reduced to every five and ten gestations years, $96 \%$ and $92 \%$ of $H_{\mathrm{E}}$ averaged over 10 microsatellite loci was maintained respectively (Table 4.6; Figures 4.3 C-D). Supplementations made with one pair (1F, 1M) of Zimbabwe rhino every one, two, five and 10 gestational years increased the mean $H_{\mathrm{E}}$ by 34\%, 27\%, 31\%, and 29\% every 96 BRGs (1000 gestational years) (Table 4.6; Figures $4.3 \mathrm{~A}-\mathrm{D})$.

The difference in variation between the non-supplemented model population with a $430 \mathrm{CC}$ and the model supplemented with one pair of KZN $D$. b. minor every one, two, five and 10 years is $32 \%, 30 \%, 25 \%$ and $19 \%$ respectively after 96 BRGs (1000 gestational years) (Tables $4.4 \& 4.6)$. When the number of individuals supplemented to the model population from the KZN metapopulation was increased to two pairs (2F, 2M), every one, two, five and ten gestational years, there was only a slight improvement in the increase in genetic variation over the single pair scenarios (Table 4.6, Figure 4.4A). However, similar to the single pair, supplementations made with individuals from the native Zimbabwe population, increasing the pair number to two greatly improved the level of diversity within the model population (Table 4.6, Figure 4.4B). 


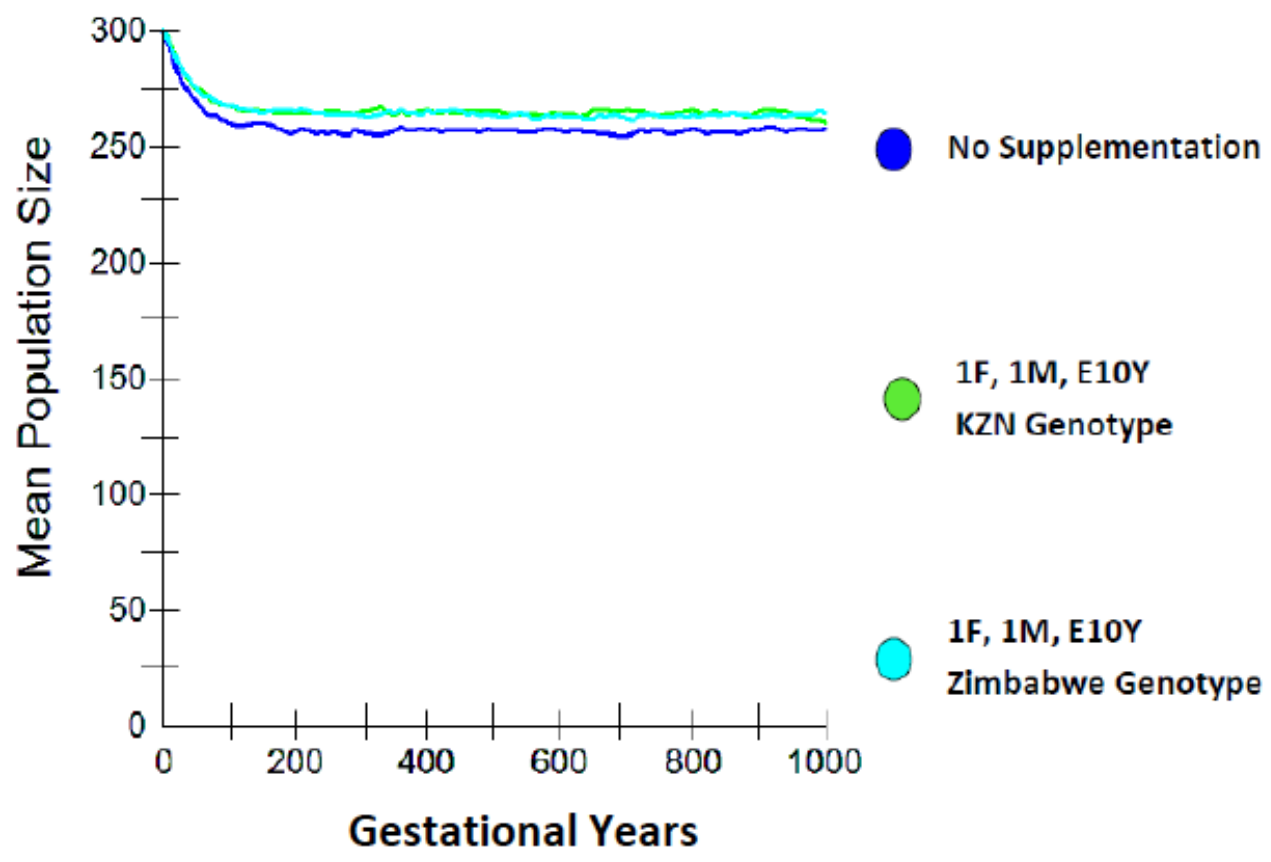

Figure 4.2: The mean population sizes for a modelled population with a CC of 430 , without inbreeding depression, with a $4 \%$ harvesting rate (translates to $5.4 \%$ in a calendar year) with and without supplementations. Supplementations were made with one female and one male $(1 \mathrm{~F}, 1 \mathrm{M})$ every ten years (E10Y; 13.4 years for a calendar year). Supplemented individuals were either assigned the KZN genotype or the native $D$. b. minor Zimbabwe genotype respectively. 
Table 4.6: Mean population sizes and expected heterozygosity $\left(H_{E}\right)$ with standard error for a population of $D$. $b$. minor; with various

supplementations; without inbreeding depression at 430 carrying capacity for years $50,100,150,200,250,500,700 \& 1000(5,10,14,19,24,48$ 67 , and 96 black rhino generations respectively). The $4 \%$ harvest is for a gestational year, which translates to $5.4 \%$ in a calendar year.

Supplementations (Supp.) were made with one male and one female $(1 \mathrm{~F}, 1 \mathrm{M})$, two females and two males $(2 \mathrm{~F}, 2 \mathrm{M})$ at intervals of every one year (E1Y), two years (E2Y), five years (E5Y) and 10 years (E10Y) with individuals with the KwaZulu-Natal genotype (KZN GT) and Zimbabwe genotype (Zim. GT).

\begin{tabular}{|c|c|c|c|c|c|c|c|c|c|c|c|}
\hline \multicolumn{3}{|c|}{ Supp. w/ 1F, 1M, E1Y - KZN GT } & \multicolumn{3}{|c|}{ upp. w/ 1F, 1M, E1Y - Zim. GT } & \multicolumn{3}{|c|}{ upp. w/ 2F, 2M, E1Y - KZN GT } & \multicolumn{3}{|c|}{ Supp. w/ 2F, 2M, E1Y - Zim. G } \\
\hline ar & $\begin{array}{c}\text { Mean Pop. } \\
\text { Size }\end{array}$ & Mean $H_{\mathrm{E}}$ & ear & $\begin{array}{c}\text { Mean Pop. } \\
\text { Size }\end{array}$ & le & par & $\begin{array}{c}\text { Mean Pop. } \\
\text { Size }\end{array}$ & & $\ldots$ & $\begin{array}{c}\text { Mean Pop. } \\
\text { Size }\end{array}$ & - \\
\hline 50 & $328 \pm 1.10$ & 461 & 50 & $326 \pm 1.12$ & $605 \pm$ & 50 & $356 \pm 0.83$ & 462 & 50 & $358 \pm 0.85$ & $0.644 \pm 0.0$ \\
\hline 100 & $9 \pm 1.04$ & & & $328 \pm 1.06$ & & & $358 \pm 0.81$ & & 100 & & \\
\hline & & & & & & & & & 50 & & \\
\hline 200 & & & 200 & & & & & & 00 & & \\
\hline 250 & & & & & $0+$ & & \pm 0.86 & & 250 & $359 \pm 0.82$ & \\
\hline & & & & & & & & & & & \\
\hline 700 & & & 700 & & & 700 & & & 700 & & \\
\hline 1000 & \pm 1.08 & & 1000 & & & & & & 1000 & & \\
\hline \multicolumn{3}{|c|}{ Supp. w/ 1F, 1M, E2Y - KZN GT } & \multicolumn{3}{|c|}{ Supp. w/ 1F, 1M, E2Y - Zim. GT } & \multicolumn{3}{|c|}{ Supp. w/ 2F, 2M, E2Y - KZN GT } & \multicolumn{3}{|c|}{ Supp. w/ 2F, 2M, E2Y - Zim. GT } \\
\hline ear & $\begin{array}{c}\text { Mean Pop. } \\
\text { Size }\end{array}$ & Mean $H_{\mathrm{E}}$ & Year & $\begin{array}{c}\text { Mean Pop. } \\
\text { Size }\end{array}$ & ( & 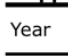 & $\begin{array}{c}\text { Mean Pop. } \\
\text { Size }\end{array}$ & Mean $H_{\mathrm{E}}$ & $\ldots$ & $\begin{array}{c}\text { Mean Pop. } \\
\text { Size }\end{array}$ & 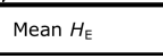 \\
\hline 50 & $300 \pm 1.31$ & & 50 & & & 50 & & & 50 & & \\
\hline 100 & & & 100 & & & 100 & & & 100 & & \\
\hline 150 & 25 & & 150 & & & 150 & & & 150 & & \\
\hline & & & & & & & & & 00 & & \\
\hline 250 & 2 & & & & & 250 & & & 50 & & \\
\hline & & & & & & 00 & & & 0 & & \\
\hline & & & & & & & & & 0 & & \\
\hline & & & & & & 1000 & & & 00 & & \\
\hline \multicolumn{3}{|c|}{ Supp. w/ 1F, 1M, E5Y - KZN GT } & \multicolumn{3}{|c|}{ Supp. w/ 1F, 1M, E5Y - Zim. GT } & \multicolumn{3}{|c|}{ Supp. w/ 2F, 2M, E5Y - KZN GT } & \multicolumn{3}{|c|}{ Supp. w/ 2F, 2M, E5Y - Zim. GT } \\
\hline ar & $\begin{array}{c}\text { Mean Pop. } \\
\text { Size }\end{array}$ & Mean $H_{E}$ & Year & $\begin{array}{c}\text { Mean Pop. } \\
\text { Size }\end{array}$ & Mean $H_{\mathrm{E}}$ & Year & $\begin{array}{c}\text { Mean Pop. } \\
\text { Size }\end{array}$ & Mean $H_{\mathrm{E}}$ & ar & $\begin{array}{c}\text { Mean Pop. } \\
\text { Size }\end{array}$ & \\
\hline 50 & $281 \pm 1.30$ & & 50 & .28 & & 50 & $294 \pm 1.31$ & & $\overline{50}$ & $5 \pm 1.29$ & \\
\hline & & & & & & & & & & & \\
\hline & & & & & & & & & & & \\
\hline 200 & & & & & & & & & 00 & & \\
\hline & & & & & & & & & & & \\
\hline & & & & & & & & & & & \\
\hline & & & & & & & & & 0 & & \\
\hline & & & & 27 & 0.5 & & 29 & 0.4401 & 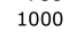 & 26 & 01 \\
\hline \multicolumn{3}{|l|}{ Supp. } & \multicolumn{3}{|c|}{ Supp. w/ 1F, 1M, E10Y - Zim. G1 } & \multicolumn{3}{|c|}{ Supp. w/ 2F, 2M, E1OY - KZN GT } & \multicolumn{3}{|c|}{ Supp. w/ 2F, 2M, E10Y - Zim. } \\
\hline ar & $\begin{array}{c}\text { Mean Pop. } \\
\text { Size }\end{array}$ & & Year & $\begin{array}{c}\text { Mean Pop. } \\
\text { Size }\end{array}$ & & Year & $\begin{array}{c}\text { Mean Pop. } \\
\text { Size }\end{array}$ & & Year & $\begin{array}{c}\text { Mean Pop. } \\
\text { Size }\end{array}$ & Mean $H_{E}$ \\
\hline 5 & $275 \pm 1.33$ & & & & & & 30 & & & 1.28 & \\
\hline 100 & 2 & & & 2 & 0 & & 5 & & 00 & 24 & \\
\hline & & & & & & & & & & & \\
\hline & & & & & & & & & & & \\
\hline 250 & 2 & & & 2 & 0 & & 22 & 0 & 50 & \pm 1.29 & 01 \\
\hline & & & & & & & & & & & \\
\hline 700 & & & & & & & & & & .29 & \\
\hline (nos & 22 & $\pm c$ & & 25 & \pm & 0 & $275 \pm$ & $34 \pm$ & & 27 & .58 \\
\hline
\end{tabular}



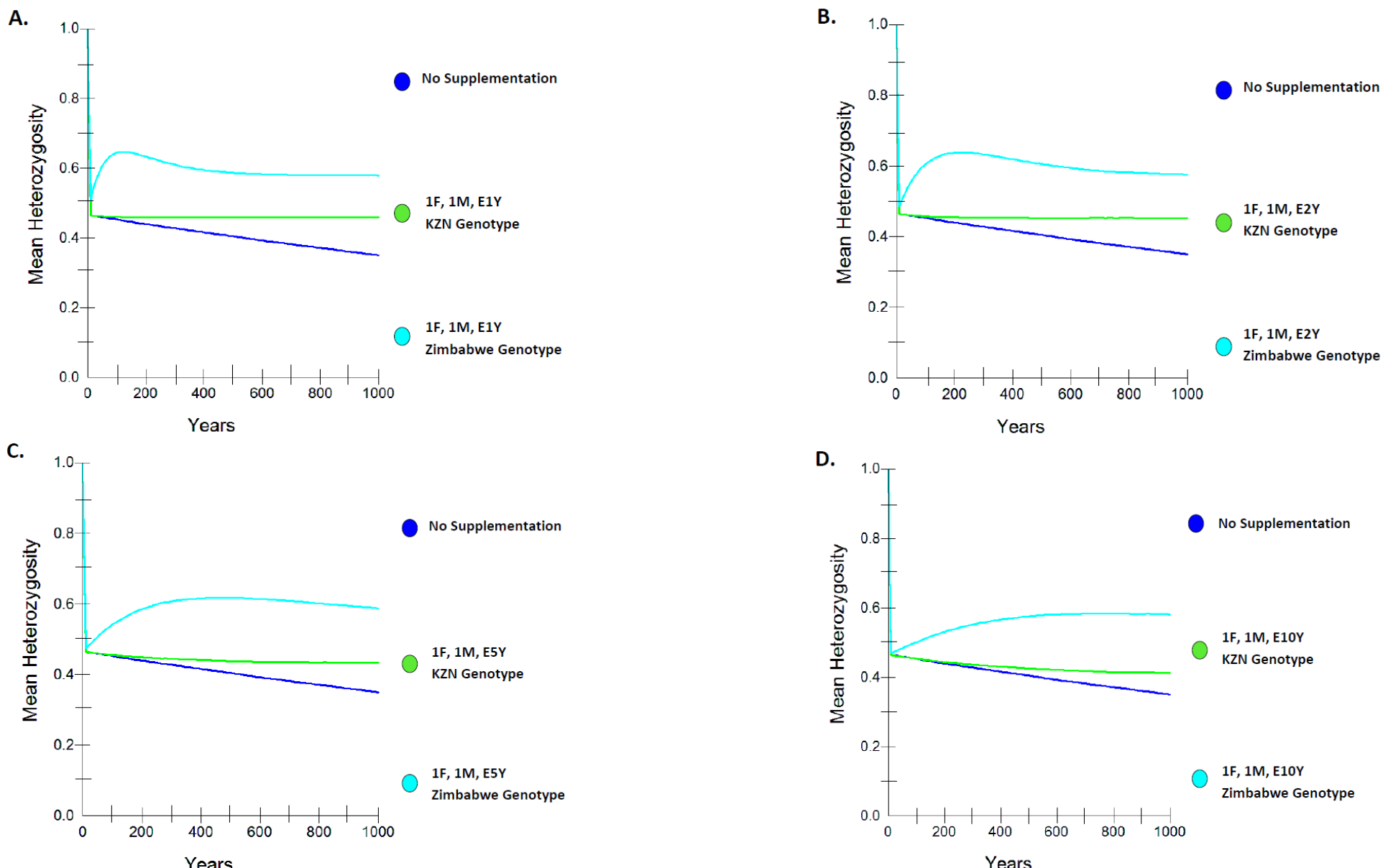

Figure 4.3: Mean expected heterozygosity for a modelled population with a carrying capacity of 430, without inbreeding depression, with a $4 \%$ harvesting rate per gestational year (translates to $5.4 \%$ in a calendar year) with and without supplementations. Supplementations were made with one female and one male (1F, 1M). Supplemented individuals were either from the KZN metapopulation or the native D. b. minor Zimbabwe population. A) Supplementations made every gestational year (1EY). B) Supplementations made every two gestational years (E2Y). C) Supplementations made every five gestational years (E5Y). D) Supplementations made every 10 gestational years (E10Y) 
A.

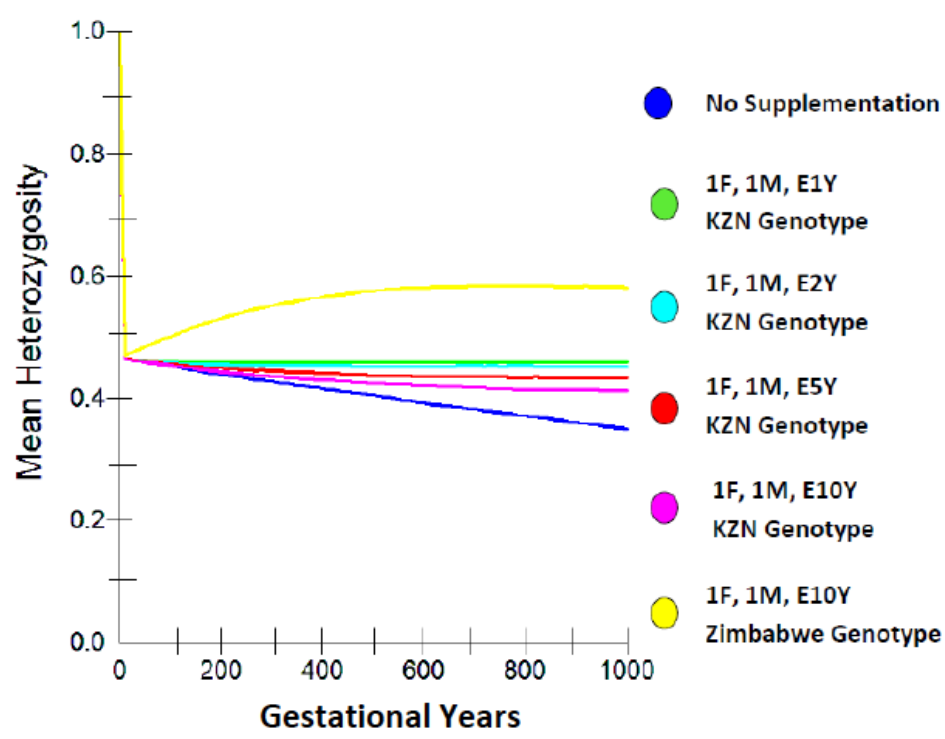

B.

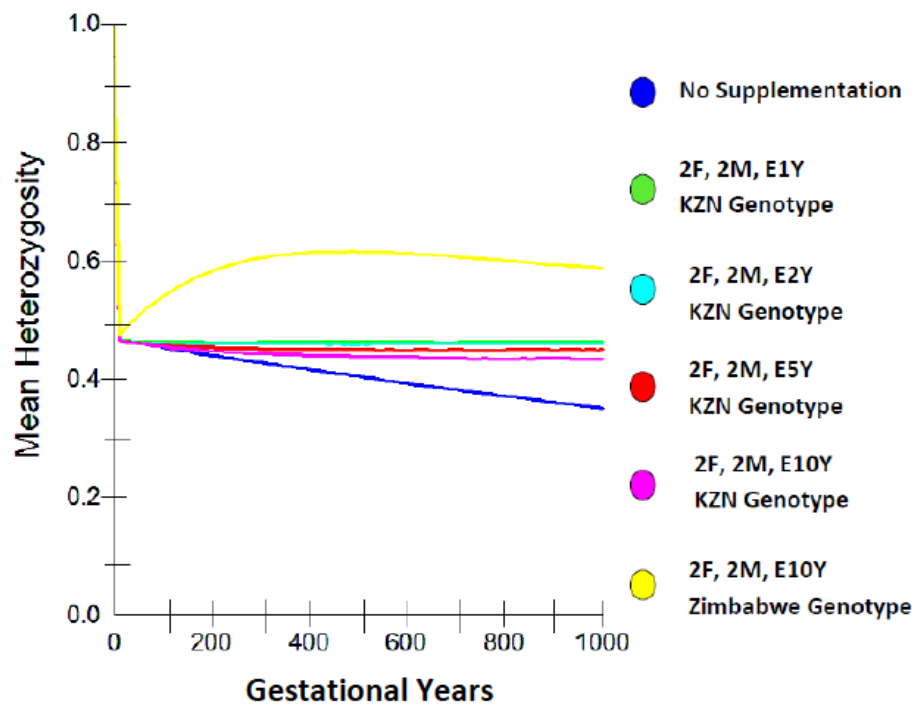

Figure 4.4 Mean expected heterozygosity for a modelled population with a CC of 430 , without inbreeding depression, with a $4 \%$ harvesting rate per gestational year (translates to $5.4 \%$ in a calendar year) with and without supplementations. Supplemented individuals were either from the KZN metapopulation or the native $D$. b. minor Zimbabwe metapopulation. A. Supplementations made with one female and one male (1F, 1M) every one (E1Y), two (E2Y), five (E5Y) and ten (E10Y) gestational years. B. Supplementations were made with two females and two males (2F, 2M) every one (E1Y), two (E2Y), five (E5Y) and ten (E10Y) gestational years. 


\subsection{Discussion}

The PVA analysis showed that the population size could be increased to the level needed to prevent the loss of genetic variation by expanding the size of the reserve or through supplementations. Doubling the model population size

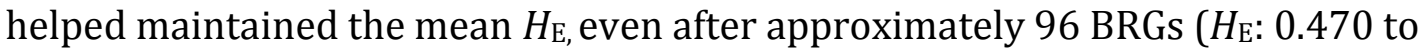
0.402 averaged over 10 microsatellite loci; 1000 gestational years /1342 calendar years), which was better than the significant loss seen when the population remained at $430 \mathrm{CC}$ over the same time period $\left(H_{\mathrm{E}}=0.470\right.$ to 0.347 averaged over 10 microsatellite loci; 1000 gestational years /1342 calendar years). Nevertheless, models also indicate that when supplementations were made with individuals from the KZN metapopulation the mean $H_{\mathrm{E}}$ improved more effectively than increasing the population size, even when supplementations only took place every ten gestational years (13.4 calendar years). However, the greatest effect on slowing the loss and increasing the mean $H_{\mathrm{E}}$ was when supplementations were made with native $D . b$. minor Zimbabwe individuals.

Currently, no translocations are made back into HiP. The only way to increase the size of the population is to expand the reserve beyond its current boundaries, which can be expensive and not always feasible. The SADC RMG supports sourcing same-subspecies individuals for founding populations from different original genetic sources including more than one source population and/or country (Emslie et al. 2009), which has been done successfully in Zambia with 25 D. b. minor sourced from several populations in South Africa (e.g. Kruger National Park, Eastern Cape, HiP, Markarele)(Chomba and Matandiko 2011). Guidelines do not, offer recommendations specifically for 
supplementing source populations. I recommend that this gap in guidelines for managing source populations be addressed, especially when populations are unable to expand and have no natural or assisted immigration. Increasing the number of black rhino in HiP could slow the loss of genetic variation. No decrease in genetic variation has been found in small populations that experienced rapid population expansion in habitats with virtually unlimited CCs (e.g. 13 European rabbits (Oryctolagus cuniculus) imported to Australia in 1859; Zenger et al. 2003). Increasing the number of black rhino in the population can be done either by (1) physically increasing the size of the reserve allowing the CC to increase, there by increasing the effective size $\left(N_{e}\right)(2)$ using other reserves to mimic expansion by performing serial translocations between $\mathrm{HiP}$ and the metapopulation or other sources of D. b. minor. In this way, D. b. minor are both emigrating from and immigrating to HiP and the population can take advantage of an artificial increase, thereby limiting further deterioration of genetic variation.

Increasing black rhino numbers by increasing the physical size of $\mathrm{HiP}$ will probably help to decrease the rate of loss of genetic diversity, but it will not increase variation as effectively as genetic supplementation. Furthermore, the limits associated with increasing the size of the reserve (e.g. land acquisition, facilitating corridors) make this option less appealing to conservation managers. Supplementations on the other hand are relatively inexpensive by comparison and translocations in KZN occur on an annual basis, so this method could be easily incorporated into existing management plans.

Emslie (1994) states that there is no proof that a long-term decrease in heterozygosity of black rhinos will automatically diminish future performance. 
While it is true that no obvious signs of inbreeding depression within HiP have been reported, the single mtDNA haplotype and 30\% lower microsatellite DNA variation than native Zimbabwe D. b. minor and lower growth rates compared to other D. $b$. minor populations suggests that a precautionary approach to managing HiP may be necessary. Preserving current levels of genetic variation within $\mathrm{HiP}$ is important and by avoiding further genetic decay HiP can continue to improve as a source for restocking other populations demographically and genetically. An example of this was observed in an isolated population of Scandinavian grey wolves (Canis lupus: Vila et al. 2003). A single male immigrant led to an increase in the mean $H_{\mathrm{E}}$ from 0.49 to 0.62 of the small population $(n=16)$ over a five-year period (Vila et al. 2003). The HiP population is much larger than the wolf example, but a similar change can be seen in the model population using supplemented individuals from the native D. b. minor Zimbabwe population.

It is clear from the PVA results that supplementation was more effective at arresting the rate of loss of genetic variation than maintaining the $\mathrm{CC}$ at current levels or increasing the size of the reserve. However, deciding whether to use black rhino from the KZN metapopulation or native Zimbabwe D. b. minor for supplementations is not straightforward. Unfortunately, it is unknown if the low levels of mtDNA and microsatellite DNA variation in HiP were anthropogenically induced or a result from a long-term demographic separation that caused historically small population sizes and local adaptation (Chapters Two and Three). If the population has always had low levels of variation, then supplementations with individuals from the KZN metapopulation would be recommended to avoid possible outbreeding depression that could compromise 
the population's evolutionary potential. However, if it can be proven that the low variation is a consequence of overexploitation by humans and the population was continuous through Zimbabwe, then supplementations with native Zimbabwe D. b. minor would be recommended to restore genetic variation possibly to near pre-decline levels. The only way to answer this question is by examining samples of KZN black rhino from before the decline. PVAs model the best-case scenario but wild populations do not necessarily respond in the same way as a simulated population.

\subsection{Conclusion}

Detecting losses in the level of genetic diversity in an endangered source population like HiP calls attention to the importance of developing conservation strategies that prevent such losses in other endangered species. While, managing genetic risks by facilitating gene flow with minimal intervention is preferable, at this time expanding park borders is not a viable option for HiP. The HiP source population only has one mtDNA haplotype $(n=65)$ (AndersonLederer et al. 2012) an $H_{\mathrm{E}}$ of 0.47 , has a homozygote excess at five out of 10 microsatellite DNA loci and is out of HWE (Chapter Three). In addition, it is subjected to poaching pressure. While modelled results are only as reliable as the species life-history data used to create scenarios, management has the opportunity to shift from a focus from not only increasing numbers of black rhino, but ensuring the future of the species by incorporating results from PVAs into management schemes. This could improve the genetic health of HiP black rhino in a practical and evidence-based way. PVA results suggest that pseudometapopulation expansion through supplementations are effective and are not 
required to be large or frequent (one female and one male every BRG). The supplementations can be accomplished inexpensively via the metapopulation using serial translocations. Most endangered large mammals no longer have the freedom to migrate, but with assistance via translocations and genetic supplementation, counteracting low levels of genetic variation that many small, fragmented populations exhibit is possible. 
Chapter IV: PVA 


\section{CHAPTER FIVE}

Managing genetic diversity in the D. b. minor metapopulation of KZN: Thesis summary and applications

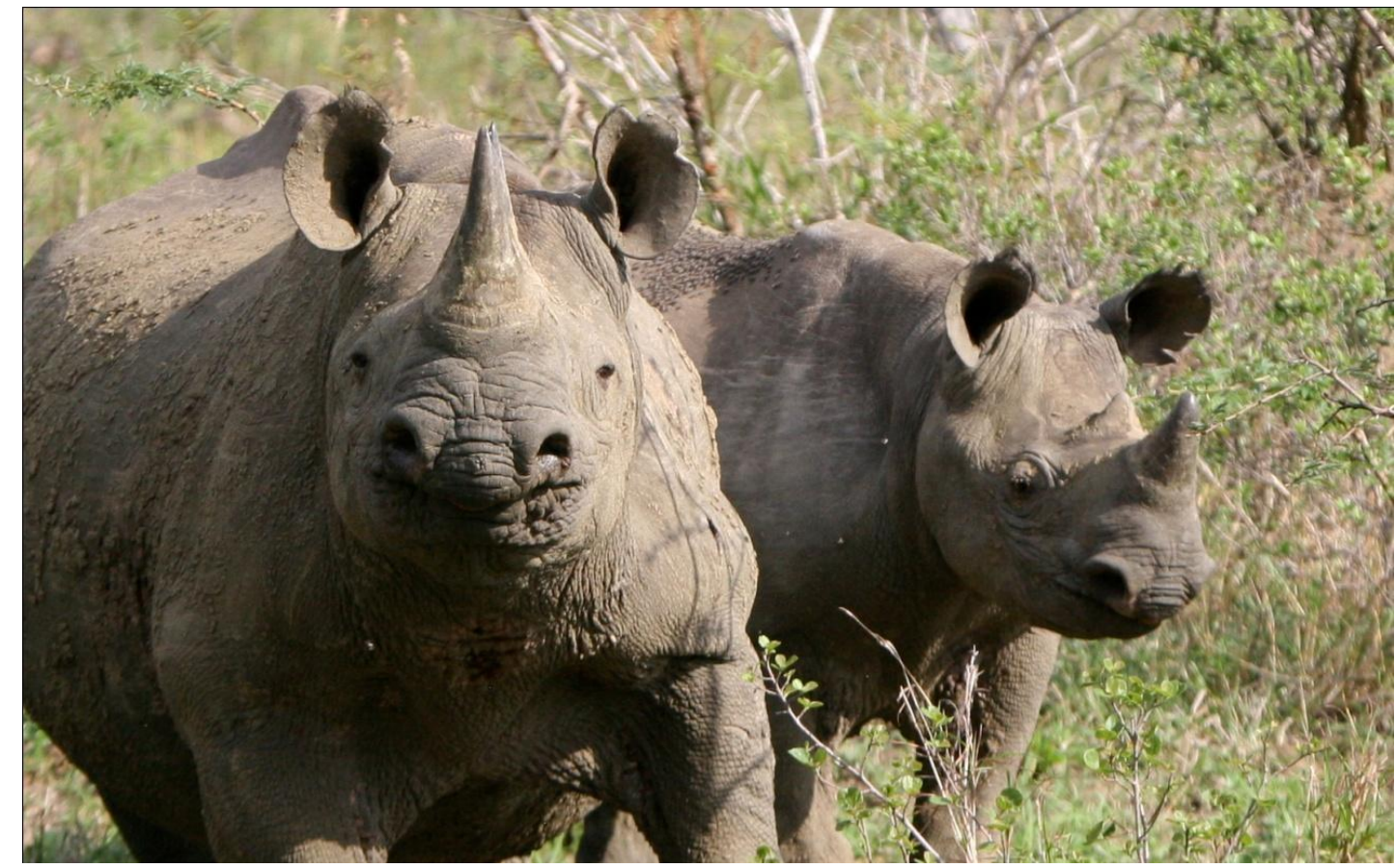

Photo by Rosalynn Anderson-Lederer 


\subsection{Introduction}

Many threatened species are now conservation-reliant after suffering severe declines in population size (see: Miller et al. 1988; Walters 1991; Tyus and Saunders 2000; Jamieson et al. 2006; Johnson et al. 2010). The continued survival of these species requires in situ and ex situ intervention by conservation teams. Unfortunately, for pragmatic reasons population genetic considerations are often not a priority when trying to increase the inevitably low number of individuals remaining in small and fragmented populations. However, when populations are in the process of recovery, wildlife managers need to shift their focus from protecting and increasing numbers to addressing population quality indicators such as the loss of genetic variation.

A loss of genetic variation in small and recovering populations could result in decreased fitness (inbreeding depression) or limit the long-term capacity of a population to respond to changes in the environment (Westemeier et al. 1998). Without shifting focus to the genetic variation in the small populations, the genetic risks may be overlooked and populations might continue to be vulnerable to extinction even though their size is increasing (Frankham 2005).

This thesis research contributes to a broader understanding of the genetic structure of the metapopulation of D. b. minor in KwaZulu-Natal (KZN), South Africa. It also demonstrates how management can incorporate a simple supplemental regime in a source population, like Hluhluwe-iMfolozi Game Park (HiP), to maintain and/or increase levels of genetic variation within the endangered, but recovering metapopulation. 


\subsection{Summary of findings}

\subsubsection{Chapter Two:}

Anderson-Lederer, R.M., Linklater, W.L., and P.A. Ritchie (2012) Limited mitochondrial DNA variation within South Africa's black rhino (Diceros bicornis minor) population and implications for management. African Journal of Ecology 50(4): 404-413. (Appendix A)

Mitochondrial DNA (mtDNA) control region sequences (406 bp) were examined to determine the level of variation within the KZN D. b. minor source population at $\mathrm{HiP}(\mathrm{n}=50)$ and KZN metapopulation $(\mathrm{n}=15)$ compared to D. $b$. minor populations outside South Africa ( $\mathrm{n}=11$; native Zimbabwe populations) and the two other black rhino subspecies (D. b. michaeli $\mathrm{n}=21, D$. b. bicornis $\mathrm{n}=4)$. The KZN source (HiP) and metapopulation had a single haplotype. However, six different haplotypes were represented in the 11 native $D . b$. minor individuals from Zimbabwe. The D. b. michaeli samples $(\mathrm{n}=21)$ had 13 haplotypes and the $D$. b. bicornis samples $(\mathrm{n}=4)$ had one haplotype. The single mtDNA haplotype in the KZN source and metapopulation coupled with previously published low levels of D. b. minor mixed population (KZN and native Zimbabwe) microsatellite DNA suggest a recent population decline and fragmentation. Small population numbers combined with fragmentation could have increased the rate of drift and may be responsible for the lack of haplotype diversity within the KZN HiP source and metapopulation. Further investigation of the native Zimbabwe $D . b$. minor mtDNA should be considered because the KZN haplotype may be present, but not yet detected. If the KZN haplotype is found in the Zimbabwe populations, management may also want to explore the possibility of 
outbreeding the native Zimbabwe $D . b$. minor populations with the translocated KZN D. b. minor population in Malilangwe, Zimbabwe.

\subsubsection{Chapter Three:}

Low levels of microsatellite DNA variation and possible management considerations for black rhino (Diceros bicornis minor) in KwaZulu-Natal, South Africa (In prep)

Ten microsatellite DNA loci were examined in the three D. bicornis subspecies: D. b. minor from the KZN source and metapopulation $(\mathrm{n}=118)$, D. $b$. michaeli $(\mathrm{n}=3)$ and $D$. b. bicornis $(\mathrm{n}=6)$. Results were compared with previously published findings for microsatellite DNA loci from a native Zimbabwe $D . b$. minor populations. The KZN source and metapopulation was out of HardyWeinberg Equilibrium and showed excess homozygosity at five loci. Direct comparison of 10 microsatellite DNA loci results confirmed that the South African metapopulation has lower genetic variation than the native Zimbabwe D. b. minor populations $\left(H_{\mathrm{E}}: 0.47\right.$ and 0.65 respectively, and lower number of alleles per locus), further indicating that current conservation plans may need to be modified to prevent additional genetic decay within KZN.

Additional loss of genetic variation and the possible risk of inbreeding depression could be prevented by rapidly increasing population numbers by increasing reserve sizes. Since procuring enough land to increase populations to sizes that would enable rapid growth is expensive and not always feasible, managers may consider serial translocations between HiP and other KZN reserves to replicate immigration and emigration. Moving rhino back into HiP would enable the source to take advantage of growth in other reserves and 
would essentially reinstate a single large genetic population. However, since native Zimbabwe D. b. minor have higher levels of genetic variation (mitochondrial and microsatellite DNA loci) than the KZN D. b. minor, managers could also consider genetic replenishment using native Zimbabwe individuals as supplements.

\subsubsection{Chapter Four:}

Population Viability Analysis of Black Rhino (Diceros bicornis minor) in Hluhluwe-iMfolozi Game Park, KwaZulu-Natal, South Africa

The KZN D. b. minor source and metapopulation have low mitochondrial DNA (Chapter Two) and microsatellite DNA (Chapter Three) variation. It was proposed in Chapters Two and Three that increasing the size of the HiP source to accommodate rapid growth may slow the rate of genetic drift and help to maintain current levels of genetic variation. It was also suggested that supplementation with individuals from the KZN metapopulation or native $D . b$. minor Zimbabwe populations may be equally effective at preserving current levels of genetic variability. To test whether one or both of the recommendations are viable options, a population viability analysis (PVA) was conducted.

The PVA modelled population increases and supplementations into HiP with individuals from the KZN metapopulation and Zimbabwe. With no change in management strategies, the PVA predicted a progressive loss in the mean expected heterozygosity of $23 \%$ over 96 black rhino generations (BRGs). Opportunities to increase the HiP population size by connecting the reserve 
through corridors to neighbouring reserves was modelled by increasing the carrying capacity (CC) of the population. Doubling the CC helped to decrease the rate of loss of the mean $H_{\mathrm{E}}$ by $11 \%$ over 96 BRGs. When supplementations of one female and one male black rhino from the KZN metapopulation were made every ten gestational years, the mean $H_{\mathrm{E}}$ of the population was maintained $\left(H_{\mathrm{E}}\right.$ 0.45) over 96 BRGs, but increased $29 \%$ when supplemented with native $D . b$. minor from Zimbabwe.

The PVA results show that serial translocation between populations is a powerful tool that can be used to decrease the rate of loss of genetic variability. In addition, it does not require a large number of individuals or to be frequent and corresponds with current management practices.

\subsection{Conservation Implications}

Data obtained in this study will allow field managers to forecast the likely changes in the levels of genetic variation within the relict HiP source population. Genetic variation in a population arises through mutation or gene flow and is typically lost either passively through genetic drift or actively through natural selection (Amos and Harwood 1998). The rate of loss of genetic variation in a population depends on its effective population size $\left(\mathrm{N}_{\mathrm{e}}\right)$ and the amount of time (number of generations) that the population has been isolated (Frankham 1997; Palstra and Ruzzante 2008). The loss of genetic variation caused by genetic drift increases in a population that has gone through a bottleneck and strong drift will continue to erode genetic variation if a population's size is unable to increase (Nei et al. 1975; Allendorf and Leary 1986b). Bottlenecks occur across many taxa, but origin, severity and population recovery times vary (i.e. they can 
develop rapidly over a short period of time in small populations or take several generations in large populations) (England et al. 2003).

Heterozygosity and allelic diversity (also called allelic richness) in populations are correlated when there is a mutation-drift balance, but after a short-term bottleneck correlation is disrupted because heterozygosity is only slightly affected (Allendorf 1986). Rare alleles on the other hand are typically lost when population sizes decrease, which means allelic diversity is expected to decrease faster than heterozygosity (Allendorf 1986; England et al. 2003).

The low levels of heterozygosity and allelic diversity, and the similar $F_{\mathrm{ST}}$ and $R_{\mathrm{ST}}$ results in HiP compared to native Zimbabwe $D . b$. minor are indicative of a recent (although not necessarily within $2 \mathrm{~N}$ to $4 \mathrm{~N}$ generations) decrease in HiP's population size. Recent bottlenecks have also been linked to reduced or low levels of genetic variation in other rhino taxa (Merenlender et al. 1989; Dinerstein and Mccracken 1990; Harley et al. 2005; Fernando et al. 2006), which is not unexpected given the high level of poaching that has occurred during the last century.

Regardless of whether the decreased level of genetic variation in the relict D. b. minor HiP population is due to the $20^{\text {th }}$ century bottleneck or historic demographic separation, it is necessary to take corrective action to prevent any further loss. The HiP population is small, fenced and has had no immigration since the latter half of the $19^{\text {th }}$ century (Swart et al. 1994), yet it is used as a source for D. b. minor subspecies expansion. HiP is also subjected to an annual harvest that targets removal of $\sim 5 \%$ of the total population each year. The removals are meant to keep the population below its CC, but this practice ultimately stagnates the size of the population, which could be having 
detrimental effects on the level of genetic variation.

This study has demonstrated that despite D. b. minor numbers increasing, $\mathrm{HiP}$ is genetically a relatively small population $(\mathrm{n}=\sim 220$ Clinning et al. 2009) and genetic drift has most likely caused genetic variability to be lost faster than would be expected in what might appear to be a larger metapopulation. Managers of any small, endangered source population should evaluate conservation plans once a species is classified as in recovery, to evaluate the levels of genetic variation and adjust priorities from quantity (number of individuals) to quality (evolutionary potential).

Using serial translocations and supplementations to maintain the levels of genetic variation in the relict HiP source population has important implications. Mangers of other small, remnant populations of conservation reliant species may be able to avoid the expense of acquiring land to 'expand' their population, by using serial translocations and supplementation to increase gene flow. Supplementation proved to be an effective tool for a remnant population of greater prairie chickens (Tympanuchus cupido pinnatus) that experienced 35 years of population declines and a reduction in genetic diversity (Westemeier et al. 1998). Despite three decades of intensive management that focused on and successfully increased the number of individuals in the population, the mean population fitness decreased to alarming levels and it was only turned around when the remnant population was supplemented with individuals from a larger population with higher levels of genetic variation (Westemeier et al. 1998).

With regards to genetic supplementation of new and established populations, management of African rhinos (black and white), the SADC RMG 
recommend that cohorts used for supplementations consist of individuals with "as little genetic similarity with the receiving population as possible" (Emslie and Brooks 1999). They also recommend that when establishing new populations, animals should not be from the same genetic source (Emslie and Brooks 1999) sensu North Luangwa National Park, Zambia (Chomba and Matandiko 2011) and Kruger National Park, South Africa (Hall-Martin and Knight 1994). These recommendations are important because if individuals with similar genotypes are used to establish or augment populations it could have a negative impact on fitness and long-term evolutionary potential of the receiving population (Eldridge et al. 1999; Moritz 2002).

Rhino guidelines encourage the mixing of populations of the same subspecies (du Toit 2006b; Emslie and Brooks 1999), however, there are instances of black rhino management taking measures to prevent the same subspecies from different regions from intermixing (i.e. the 27 KZN D. b. minor translocated to Malilangwe, Zimbabwe in 1997 that are not outbred with native Zimbabwe black rhino). There is a risk of over splitting the subspecies if $D . b$. minor populations (KZN and native Zimbabwe) are treated as separate subspecies. Managing D. b. minor as one large metapopulation (e.g. similar to the translocations into Krueger National Park from South Africa and Zimbabwe) across borders may aid with the overall management goals of retaining genetic diversity, but may not be feasible due to manpower (e.g. needed for antipoaching) and budget constraints (e.g. small GDP economies). 


\subsection{Future research directions}

\subsubsection{Historic Samples}

Determining whether the current levels of genetic diversity in an endangered species population are significantly different from historic levels is difficult because it requires 'before and after' samples of the population (Leberg 2002). If both historic and current samples could be obtained, it would confirm whether or not a decline in the level of genetic variation has occurred (Roy et al. 1994). Without temporal genetic data, assessments of the amount of genetic diversity lost in populations can only be inferred by comparing them to examples of closely related species, or populations of the same species in different geographic locations (Leberg 2002).

Sampling historic levels of genetic variation may also provide some insight into the past population structure and the amount of fragmentation that has occurred. Frankham et al. (2002) described five fragmented population structures (Figure 5.1) that have various impacts on levels of variation within populations ranging from negligible to severe, depending on the structure of and migration patterns between the fragments. Identifying the historic pattern of fragmentation could help managers plan metapopulations and allow duplication of past gene flow patterns through the creation of corridors or through translocations.

Information derived from historic samples could also reveal that very little loss in the level of genetic variation has occurred and that the populations were always small and isolated. These findings would assist when implementing management practices that might lead to outbreeding depression 
Figure 5.1: The five structures of fragmented populations. (From Frankham et al. 2002)

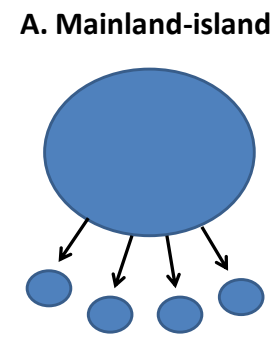

D. Two-dimensional stepping stone

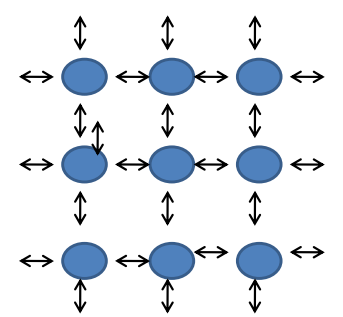

B. Island

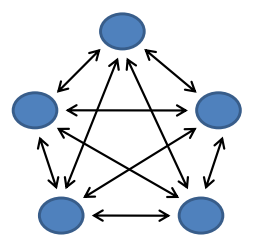

E. Metapopulation

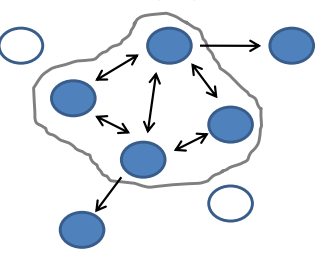

(Leberg 2002). In the case of rhino, historic samples might be obtained through zoo collections from the progeny of individuals originally captured in populations that have since been extirpated. They might also be collected from museum samples if documentation regarding the sample's origin is verified.

\subsubsection{Harvest for Population Growth}

As large wild populations of threatened and endangered species begin to recover, it will become necessary for managers to address limited reserve sizes and the need for harvesting populations when they reach maximum carrying capacities.

Translocations are used for increasing the viability of a species. Specifically, the technique is used to (1) move individuals between wild populations to bolster genetic heterogeneity of small populations, (2) move wild individuals to captivity (also called capture or collection) and (3) to move 
individuals or cohorts from captivity to the wild (also called reintroduction or release) (Griffith et al. 1989; Tenhumberg et al. 2004).

Apart from megaherbivores, translocations are not typically used to remove (or 'harvest') individuals from endangered populations that have reached their carrying capacity in order to stimulate continued population growth (see Appendix B for PVA results pertaining to HiP harvest rates) (Emslie et al. 2007; Emslie et al. 2009; Emslie 2001). Because the use of harvesting to facilitate breeding may become more popular as large species conservation programmes move into a recovery phase, shifting priorities to that of preserving levels of genetic variation will be important and warrants a more thorough examination (Lubow 1996).

\subsubsection{Translocation Cohort Sizes and Composition}

As translocation techniques are improved, reintroductions are becoming more important in the management of threatened species. There is little species specific information available, however, about the recommended number and composition (e.g. number of males and females, ages, parent-offspring) of individuals necessary in a cohort to minimize losses in levels of genetic diversity (Tracy et al. 2011).

Most researchers do not define what an 'acceptable' or 'adequate' number equates to in numeric terms, which makes planning the composition of cohorts for successful translocations and maintenance of levels of genetic diversity difficult for conservation managers. While Griffith et al. (1989) only refer to 'large' versus 'small' cohort sizes, they found that translocations had higher success rates among individuals and cohorts that were native game 
species (86\%), herbivores (77\%), and wild-caught individuals (75\%). Knapp and Dryer (1998) recommend using individuals that are a 'genetic match' or that already have local adaptations to the environment to improve the success rates. They found that individuals used for reintroductions that had adaptations to local conditions had higher fitness and lower mortality than individuals that were translocated to areas they were not adapted to (see Appendix C for PVA results pertaining to HiP cohort sizes).

As field managers are increasingly using translocations and reintroductions as tools for managing wild populations, improving the success of reintroductions while maintaining levels of genetic variation is an area that requires further investigation.

\subsubsection{Functionally important genetic variation and Conservation Genomics}

To successfully conserve an endangered species, it is important to: (1) identify different species within taxa, (2) identify evolutionarily significant units (ESUs) within species, and (3) identify management units (MUs) within ESUs (Moritz 1994; Hedrick et al. 2001). Examining the relative levels of genetic variation at neutral markers like mitochondrial or microsatellite DNA loci, can reveal historic population characteristics that support ESU and MU designation (Bos et al. 2008), however maintaining adaptive variation is important to the conservation objective that supports ESU and MU designations (Hedrick et al. 2001).

While demographic factors (e.g. genetic drift and inbreeding) play a crucial role in the degree of variation in neutral genes, some functional (coding) genes are under selection (Sommer 2003). Major histocompatibility complex 
(MHC) genes are functional genes that are primarily responsible for recognizing foreign proteins, presenting them to immune cells and initiating an immune response (Piertney and Oliver 2006). MHC genes have been connected with individual fitness, population viability and evolutionary potential in changing environments, which makes them ideal for studying adaptive genetic diversity (Strand 2011). However, due to the complexity of working with MHC genes (organization of MHC loci found in model species differs from non-model species), many large-scale studies of wild populations have been hindered (Strand 2011).

MHC primers for this study (Dbminor-MHC Alpha1-2 Forward: CCTCCTCCTGCTCTCGG and Dbminor-MHC Alpha 1-2 Reverse: CCACAGCCGCCCACTTCTGG) were developed using Geneious v4.8 (Drumond et al. 2010). Remaining DNA orginally extracted for Chapters Two and Three was used with the new primer pair. The forward primer was tagged with an M-13 tag (Schuelke 2000). Polymerase Chain Reaction (PCR) amplifications using 1-2 $\mu \mathrm{L}$ of DNA template were carried out in $25 \mu \mathrm{L}$ volumes with $67 \mathrm{mM}$ Tris $\mathrm{pH} 8.8$,

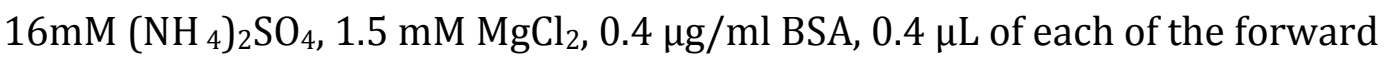
and reverse primer, $200 \mu \mathrm{M}$ of each dNTP, and 0.5 to 1 units of BIOTAQ DNA polymerase (Bioline). Thermal cycling was carried out using an Eppendorf Mastercycler for; $94^{\circ} \mathrm{C} 2 \mathrm{~min},\left(94^{\circ} \mathrm{C} 3 \mathrm{~min}, 50-54^{\circ} \mathrm{C} 30 \mathrm{sec}, 72^{\circ} \mathrm{C} 2 \mathrm{~min}\right)$, repeated for $30-40$ cycles, followed by a final step of $72^{\circ} \mathrm{C} 3 \mathrm{~min}$. PCR products were electorphoresed in agarose gel and a molecular weight standard was used to determine the size of amplified products. The resultant PCR products were analysed on a 3730 automated sequencer using the GS-500 LIZ size standard and the GENESCAN software (Applied Biosystems) I experienced significant 
difficulties amplifying MHC genes to a level of quality that would allow clear sequencing for this project.

Even though neutral and functional genes provide complimentary information about the recent evolutionary history of populations, only a small number of studies have actually used a combination of the markers to examine the relative levels of genetic variation in populations (Bos et al. 2008). Conservation genomics is a new field that utilises recent advances in DNA sequence techniques to discover genomic regions that are adaptively important for populations in particular habitats (Allendorf et al. 2010).

Most conservation genetic studies use neutral markers that are unable to answer questions about the impact of a small effective population size $\left(\mathrm{N}_{\mathrm{e}}\right)$ on functionally important genetic variation or whether microsatellite DNA variation provides a good representation of the genome wide levels of variation (Ouborg et al. 2010). Genomics will enable the field of conservation genetics to understand both the mechanistic (e.g. genetic and cellular operations and how they affect organismal development, ecology and evolution) and inventorial (functional, e.g. improve ability to take genealogical stock of biological resources at all levels in the phylogenetic hierarchy) aspects of at risk species (Avise 2010). The diagram in Figure 5.3 shows how conservation genomics can address a range of conservation genetic issues that previous techniques have been unable to address.

Conservation genetic analyses are typically based on neutral loci that only provide details of a very small part of an organism's genome, however with advances in conservation genomics (e.g. SNPs), researchers will be able to analyze genome-wide data that will translate into practical information that can 
significantly improve the way endangered species are managed. However, this new technical approach is expensive and requires detailed computational analyses, which will limit its uptake in the short term.

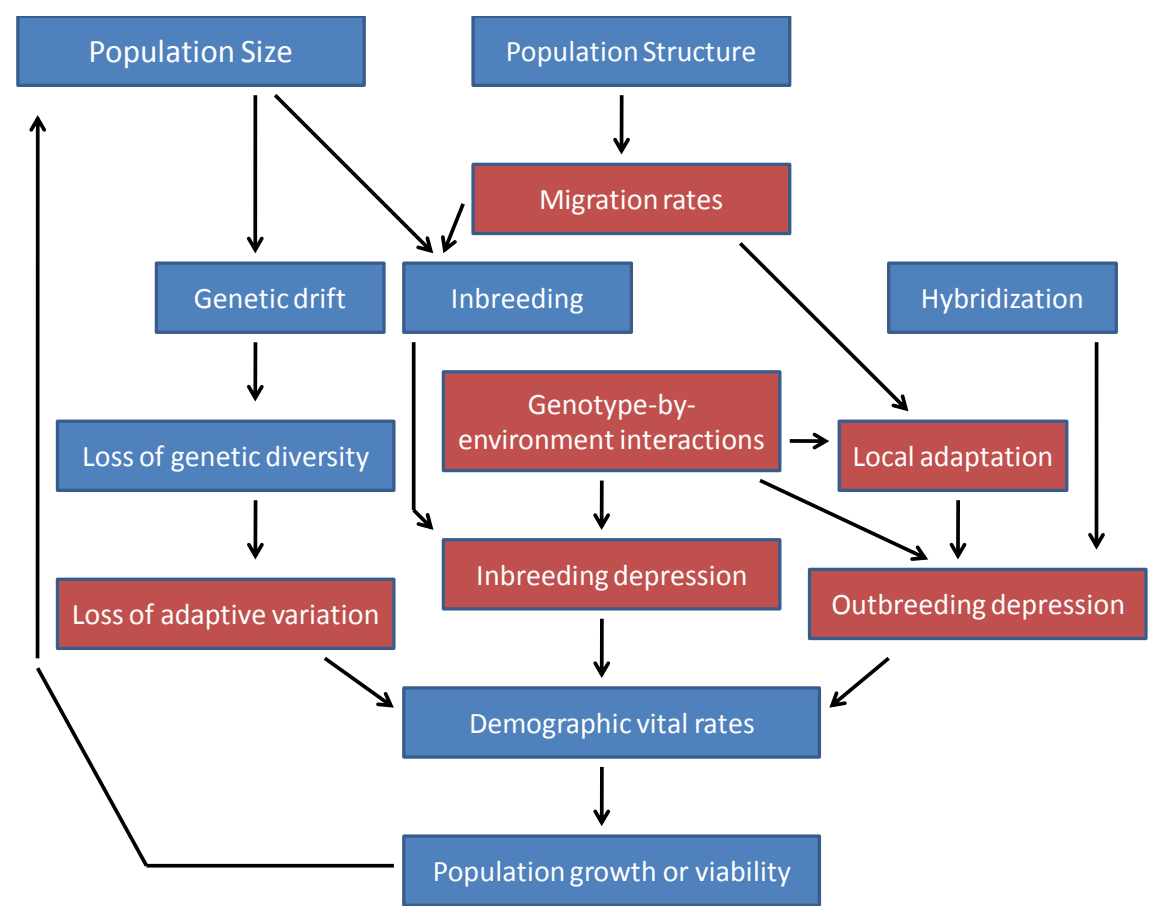

Figure 5.3: A diagram of interacting factors in the conservation of natural population. Traditional conservation genetics (neutral markers), provides direct estimates of some interacting factors (blue). Conservation genomics can address a wider range of factors (red). It also promises more precise estimates of neutral processes (blue) and understanding of the specific genetic basis of all of the factors. (From Allendorf et al. 2010)

\subsection{Summary}

In this study, I have added to the understanding of the genetic structure of the KZN remnant source (HiP) and metapopulation, highlighted genetic differences between KZN and native Zimbabwe $D$. . b. minor as well as made recommendations for maintaining the current levels of genetic heterozygosity within HiP. This is timely as the black rhino species is recovering. If poaching 
pressure can be abated, black rhino numbers may be able to match those of the southern white rhino that have been the most successful of the rhino taxa to recover from a severe bottleneck. I anticipate that this research will help to improve management of black rhino populations and contribute to conservation biology on a broader scale across species.

As rapid changes in the field continue, conservation genetics will bring to light historic and current stochastic and demographic changes occurring in wild populations. Techniques are advancing turning genetics and genomics into incredibly powerful tools for assisting in the management of populations across all levels (individual, population, and species). By wildlife managers taking research findings and incorporating them into management plans, the field of conservation genetics and genomics can help slowly reverse the process of destruction that human induced changes have caused. 


\title{
Appendix A
}

\section{African Journal of Ecology (r)}

\section{Limited mitochondrial DNA variation within South Africa's black rhino (Diceros bicornis minor) population and implications for management}

\author{
Rosalynn M. Anderson-Lederer ${ }^{1 *}$, Wayne L. Linklater ${ }^{1,2}$ and Peter A. Ritchie ${ }^{1}$ \\ ${ }^{1}$ Centre for Biodiversity and Restoration Ecology, School of Biological Sciences, Victoria University of Wellington, PO Box 600, Wellington, \\ 6041, New Zealand and ${ }^{2}$ Centre for African Conservation Ecology, Department of Zoology, Nelson Mandela Metropolitan University, PO \\ Box 77000, Port Elizabeth Eastern Cape 6031, South Africa
}

\begin{abstract}
The taxonomy of African black rhinoceros (Diceros bicornis) remains unresolved. Maintaining levels of genetic diversity and species rescue by reintroduction and restocking requires its resolution. We compared the sequences of the mitochondrial DNA (mtDNA) control region for a total of $101 \mathrm{D}$. bicornis from three subspecies: D. b. minor, $D$. b. michaeli and D. b. bicornis. A single unique haplotype was found within the 65 D. b. minor samples from KwaZulu-Natal (KZN) Province, South Africa, 55 of which came from Hluhluwe-iMfolozi Game Park (HiP) and Mkuzi Game Reserve (MGR) source populations. However, six different haplotypes were represented in eleven $D . b$. minor samples from Zimbabwe. Similarly, published autosomal microsatellite data indicate low levels of diversity within the KZN D. b. minor populations. The low levels of mtDNA diversity within the KZN metapopulation point to the possible need for genetic supplementation. However, there is a need to determine whether the low levels of genetic variation within KZN D. b. minor are a result of the recent bottleneck or whether KZN historically always had low diversity.
\end{abstract}

Key words: conservation genetics, control region, Diceros bicornis, D-loop, genetic rescue

\section{Résumé}

La taxonomie du rhinocéros noir d'Afrique (Diceros bicornis) n'est pas encore résolue. Pour préserver le taux de diversité génétique et pouvoir sauvegarder l'espèce par des réintroductions et des repeuplements, il faut résoudre cette

*Correspondence: Email: rosalynn.anderson-lederer@vuw.ac.nz question. Nous avons comparé les séquences de la région de contrôle de l'ADN mitochondrial (ADNmt) d'un total de 101 D. bicomis appartenant aux trois sous-espèces D. b. minor, D. b. michaeli et D. b. bicornis. Nous avons trouvé un seul et unique haplotype pour les 65 échantillons venant de D. b. minor de la province du KwaZuluNatal (KZN) en Afrique du Sud, dont 55 venaient de populations sources du Parc de Hluhluwe-iMfolozi (HiP) et de la Réserve de Faune de Mkuzi (MGR). Par contre, il y avait six haplotypes différents dans 11 échantillons de D. b. minor venant du Zimbabwe. Les données publiées sur les microsatellites autosomaux indiquent, elles aussi, un faible taux de diversité au sein des populations de D. b. minor du KZN. Le faible taux de diversité de l'ADNmt dans la métapopulation du KZN indique un éventuel besoin de supplémentation génétique. Cependant, il faut d'abord déterminer si le faible taux de variation génétique chez les $D$. $b$. minor du KZN est un résultat des réductions récentes ou si le KZN a toujours eu une faible diversité.

\section{Introduction}

Species conservation depends on identifying genetically distinct groups or management units and implementing strategies to retain genetic variation. Genetically distinct populations can contain unique genetic variation, and/or they can be locally adapted to their habitat. Mixing them with other populations may break up genetically complex traits and, in some cases, lead to outbreeding depression (Templeton, 1986; O'Ryan, Flamand \& Harley, 1994). Alternatively, genetic differences between populations can also result from strong genetic drift caused by population fragmentation and declining 
population sizes (Frankham, Ballou \& Briscoe, 2002; Allendorf \& Luikart, 2007). When the genetic structure and historic pattern of gene flow of a species has been described, reintroduction methods can be used to secure locally adapted populations or restocking used for genetic supplementation.

Variation in mitochondrial DNA (mtDNA) is a particularly useful metric for determining population structure and history (Moritz, 1994). The control region of mtDNA is highly variable, and it can often be used to resolve phylogenetic relationships between closely related taxa or for describing the genetic structure within species (Moritz, Dowling \& Brown, 1987; Kidd \& Friesen, 1998). MtDNA is maternally inherited and so does not recombine (Hayashi, Tagashira \& Yoshida, 1985), which means it reflects a quarter the effective population size $\left(\mathrm{N}_{\mathrm{e}}\right)$ compared with nuclear loci and hence it is more sensitive to changes in population demography.

The black rhinoceros (Diceros bicornis: Perisodactyla) once ranged across the African continent and numbered in the hundreds of thousands (Western \& Vigne, 1985). By 1969, their numbers had declined to $\sim 65,000$ (Muya \& Oguge, 2000), and during the last century, the species disappeared faster than any other large mammal (Hitchins, 1975; Western \& Vigne, 1985). The major causes for their decline have been anthropogenic, primarily illegal hunting (Western \& Vigne, 1985; Emslie \& Brooks, 1999; Amin et al., 2006). Nevertheless, conservation efforts have seen in situ black rhino numbers increase from a low of 2475 individuals in 1993 to approximately 4880 in 2010 (Emslie, 2011).

Three extant black rhino subspecies are recognized across Africa, including approximately $742 \mathrm{D}$. b. michaeli (Eastern black rhino), 1922 D. b. bicornis (South-western black rhinoceros) and 2216 D. b. minor (South-central black rhinoceros) (Emslie, 2011). Appraisal of the black rhino subspecies was initially based on skull measurements (Zukowsky, 1964; Groves, 1964; du Toit, 1987); however, uncertainty regarding taxonomy remained (du Toit, 1987). Although there are apparently no impervious geographic boundaries or reproductive barriers between the subspecies, they occupy different areas with distinct habitats and climates (Harley et al., 2005; Emslie \& Brooks, 1999). With no historical records of migration and the extent of gene flow between the subspecies unknown, some authors have speculated that each subspecies may have genetic or behavioural adaptations to their local environments (Emslie \& Brooks, 1999; Harley et al. 2005). Their suggestion regarding genetic differences was confirmed through recent mtDNA and autosomal DNA analyses (Merenlender et al., 1989; Ashley, Melnick \& Western, 1990; O'Ryan \& Harley, 1993; O'Ryan, Flamand \& Harley, 1994; Swart \& Ferguson, 1997; Brown \& Houlden, 1999, 2000; Nielsen et al. 2008; Karsten et al., 2011; Muya et al., 2011). Thus, current black rhino management policy is for each subspecies to be managed separately in order to maintain possible local adaptive traits and minimize the risk of outbreeding depression (Templeton, 1986; O'Ryan, Flamand \& Harley, 1994; Brown \& Houlden, 2000; Harley et al. 2005).

The largest remnant population of the critically endangered (IUCN, 2008) D. b. minor subspecies is in Hluhluwe-iMfolozi Game Park (HiP) ( $\mathrm{n}=\sim 220$ Clinning et al. 2009) in KwaZulu-Natal (KZN) Province, South Africa (Fig. 1). KZN black rhinos have been separated from other populations to the north (e.g. Zimbabwe) since at least the latter half of the 19th century (Swart et al. 1994). HiP and the smaller remnant in Mkuze Game Reserve (MGR) ( $\mathrm{n}=\sim 45 \mathrm{D}$. Kelly, personal communication) have been sources for metapopulation expansion and genetic management by reintroduction and restocking. Translocations from $\mathrm{HiP}$ to other KZN reserves first began in 1962, expanded to other South African provinces and later to other African nations (e.g. Zimbabwe, Zambia) (Hitchins, 1984; Emslie, Amin \& Kock, 2009). The potential now exists for KZN D. b. minor to be mixed with $D . b$. minor in or from other smaller African populations, especially those in Zimbabwe, if they are not too genetically divergent. Although the KZN population will likely be strategic to the subspecies recovery throughout the African continent (Emslie \& Brooks, 1999), no study has yet compared the mtDNA sequences of the KZN D. b. minor metapopulation with populations outside South Africa.

The aim of our study was to use mtDNA control region sequences (406 bp) to determine the level of variation within the D. b. minor source population at $\mathrm{HiP}$ ( $\mathrm{n}=50$ ) and compare it with the KZN metapopulation ( $\mathrm{n}=15$ ) and D. b. minor populations outside South Africa $(\mathrm{n}=11)$ and the other black rhino subspecies (D. b. michaeli $\mathrm{n}=21$, D. b. bicornis $\mathrm{n}=4$ ). We considered the implications of our findings for the long-term management of D. b. minor and make recommendations for possible future research. 


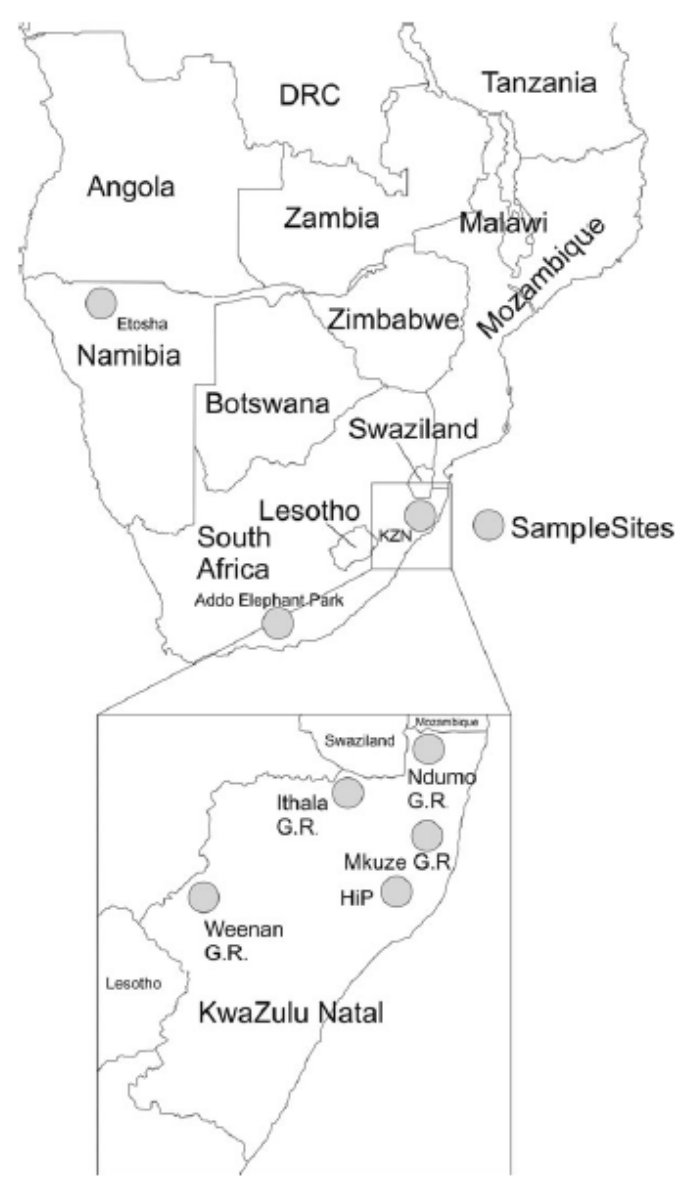

Fig 1 Map of southern Africa showing black rhinoceros sample sites. Inset showing KwaZulu-Natal study Game Reserves (Ndumo G.R., Ithala G.R., Mkuze G.R., Hluhluwe-iMfolozi Game Park (HiP) and Weenan G.R.).

\section{Materials and methods}

Sampling

Samples of blood and pinna ear tissue were collected from individuals of $D . b$. minor in the KZN province in South Africa $(\mathrm{n}=65)$, D. b. michaeli in Addo Elephant National Park, South Africa $(\mathrm{n}=1)$, and D. b. bicornis in Namibia's northern region $(n=4)$ (Fig. 1). The samples were acquired opportunistically during routine translocation and ear notching (for identification) events from 2002 to 2009. Blood samples were stored in cryovials containing $1 \mathrm{ml}$ of DMSO/EDTA/Tris/salt solution (Seutin, White \& Boag, 1991).

DNA sequencing and analysis

DNA extraction. Seventy micro-litres of the preserved blood solution or a $3 \times 3 \mathrm{~mm}$ piece of pinna ear tissue was digested in an SDS/proteinase-K solution. After dissolution, a standard phenol-chloroform DNA extraction and ethanol precipitation was conducted following the procedure of Sambrook, Fritsch \& Maniatis (1989).

PCR and DNA sequencing. A fragment of the mitochondrial DNA control region (406 bp) was amplified using the primers mt15996L (5'-TCCACCATCAGCACCCAA AGC-3') (Campbell et al., 1995; Brown \& Houlden, 2000) and mt16502H (5'-TTTG-ATGGCCCTGAAGTAAGAAC CA-3') (Moro et al., 1998; Brown \& Houlden, 2000). PCR amplifications using 1-2 $\mu \mathrm{l}$ of DNA template were carried out in $25 \mu \mathrm{l}$ volumes with $67 \mathrm{~mm}$ Tris $\mathrm{pH} 8.8,16 \mathrm{~mm}$ $\left(\mathrm{NH}_{4}\right)_{2} \mathrm{SO}_{4}, \quad 1.5 \mathrm{mM} \mathrm{MgCl}_{2}, 0.4 \mu \mathrm{g} \mathrm{ml}^{-1}$ BSA, $0.4 \mu \mathrm{l}$ of each of the forward and reverse primer, $200 \mu \mathrm{m}$ of each dNTP and 0.5-1 units of BIOTAQ DNA polymerase (Bioline USA Inc., Taunton, MA, USA). Thermal cycling was carried out using an Eppendorf Mastercycler at $94^{\circ} \mathrm{C}$ for $2 \mathrm{~min},\left(94^{\circ} \mathrm{C}\right.$ for $3 \mathrm{~min}, 50-54^{\circ} \mathrm{C}$ for $30 \mathrm{~s}, 72^{\circ} \mathrm{C}$ for $2 \mathrm{~min}$ ), repeated for $30-40$ cycles, followed by a final step of $72^{\circ} \mathrm{C}$ for $3 \mathrm{~min}$.

PCR products were electorphoresed in agarose gel, and a molecular weight standard was used to determine the size of amplified products. Products of the correct size were purified using column purification (Roche Corporate Communications, Basel, Switzerland) or ExoSAP-IT (GE Healthcare, Waukesha, WI, USA), and their DNA sequence was determined using an ABI 3730 Genetic Analyzer (Massey Genome Service, Palmerston North, New Zealand).

For comparison, eleven $D . b$. minor sequences stored in GenBank (accession numbers AF187825 - AF187827 \& AF187829 - AF187831, Brown \& Houlden, 2000; AY742832 \& AY742833, Fernando et al, 2006) originally sampled from Zimbabwe and zoos in Australia and the United States were added to the data set, in addition to 20 D. b. michaeli samples (accession numbers AF187834 \& AF187835, Brown \& Houlden, 2000; AY742830 \& AY742831, Fernando et al., 2006: FJ227484 - FJ227498, Muya et al., 2011) originally 
sampled from Kenya and zoos in Australia and the United States.

Data analysis. The 101 mitochondrial DNA sequences were edited by eye and then aligned using Clustal $\mathrm{W}$ (Larkin et al., 2007). Homogeneity of base compositions was tested using PAUP 4.10b (Swofford, 2002). DnaSP v 5.10 .1 (Rozas et al., 2003) was used to calculate haplotype diversity $(h)$, nucleotide diversity (p) and standard deviation (SD) within the subspecies. The level of sequence divergence within and between populations was estimated using a pairwise distance analysis in MEGA 5.1 (Tamura et al., 2011), and standard errors were calculated using a bootstrap procedure. A statistical parsimony haplotype network was calculated using NETWORK 4.610 (Bandelt, Forster \& Rohl, 1999).

\section{Results}

The sequence of the mtDNA control region was determined for a total of 70 individual black rhinos as follows: D. b. minor samples: 50 from HiP, eight from Itala, five from MGR, one from Ndumo Game Reserve, one from the Johannesburg Zoo (accession number JN593089) and eleven sequences from GenBank (accession numbers AF187826 - AF187831, AY742832 - AY742833 \& AF187832 - AF187833): D. b. michaeli samples: one from Addo Elephant Park (Accession number JN5930090) and 20 from GenBank (accession numbers FJ227483 - FJ227498, AY742830 - AY742831 \& AF187834 - AF187835) and four samples for D. b. bicornis from Namibia's northern region (accession numbers JN593091-JN593094) (Table 1).

The 101 aligned sequences were 363 bp long with 31 polymorphic sites, and there was an average pairwise difference of $4 \% \quad( \pm 1 \%)$ between $\quad$ D. b. michaeli and D. b. minor, $4.5 \%( \pm 1.1 \%)$ between D. b. michaeli and D. b. bicornis and $2.3 \%( \pm 0.8 \%)$ between D. b. minor and $D . b$. bicornis. No insertions or deletions were observed.

Considering each subspecies separately, the greatest level of diversity was recorded in D. b. michaeli $(\mathrm{n}=21)$, which contained thirteen haplotypes and showed comparatively high nucleotide diversity $(\mathrm{p}=0.011 \pm$ $0.00106)$ and haplotype diversity $(h=0.958 \pm 0.026)$ (Table 2). The lowest level of diversity within a subspecies was seen in the Namibian D. b. bicornis samples

Table 1 Rhinoceros subspecies and sources analysed for mtDNA variation

\begin{tabular}{|c|c|c|c|}
\hline Subspecies & $\begin{array}{l}\text { Sample } \\
\text { size }\end{array}$ & Sample/sequence source & References \\
\hline \multirow[t]{10}{*}{$\begin{array}{l}\text { Dioeros bicomis } \\
\text { minor }\end{array}$} & 50 & $\begin{array}{l}\text { Hluhluwe-iMfolozi Game Park, KNZ Region, Accession number } \\
\text { JN593089 }\end{array}$ & This study \\
\hline & 8 & Itala Game Park, KNZ Region & This study \\
\hline & 5 & Mkuze Game Park, KNZ Region & This study \\
\hline & 1 & Ndumo Game Park, KNZ Region & This study \\
\hline & 1 & Johannesburg Zoo & This study \\
\hline & 6 & $\begin{array}{l}\text { Chete National Park, Zimbabwe, Accession numbers } \\
\text { AF187825-AF187827 \& AF187829-AF187831 }\end{array}$ & Brown \& Houlden, 2000; \\
\hline & 2 & $\begin{array}{l}\text { Zambezi Valley, Zimbabwe, Accession numbers } \\
\text { AY742832-AY742833 }\end{array}$ & Femando et al., 2006; \\
\hline & 1 & Captive bom, San Diego Zoo Accession number AF187832 & Brown \& Houlden, 2000; \\
\hline & 1 & Captive bom, Milwaukee Zoo, Accession number AF187833 & Brown \& Houlden, 2000; \\
\hline & 1 & Captive bom, Western Plains Zoo Accession number AF187828 & Brown \& Houlden, 2000; \\
\hline \multirow[t]{5}{*}{ D. b. michaeli } & 16 & Kenya, Accession numbers FJ227483-FJ227498 & Muya 2011 \\
\hline & 2 & $\begin{array}{l}\text { Solio Game Reserve, Kenya, Accession numbers } \\
\text { AY742830-AY742831 }\end{array}$ & Femando et al., 2006; \\
\hline & 1 & Cincinnati Zoo, Accession number AF 187834 & Brown \& Houlden, 2000; \\
\hline & 1 & Taronga Zoo, Accession number AF187835 & Brown \& Houlden, 2000; \\
\hline & 1 & Addo Elephant Park, South Africa Accession number JN593090 & This study \\
\hline D. b. bioornis & 4 & Etosha, Namibia, Accession numbers JN593091-JN593094 & This study \\
\hline $\begin{array}{l}\text { Ceratotherium } \\
\text { simum }\end{array}$ & 4 & HiP \& London Zoo, Accession numbers AF187836-AF187839 & Brown \& Houlden, 2000 \\
\hline
\end{tabular}




\begin{tabular}{lrrrrrlll}
\hline & \multicolumn{7}{c}{ Genetic variability } \\
\cline { 5 - 8 } & $\mathrm{n}$ & $\mathrm{H}$ & $\mathrm{h}$ & (SD) & \multicolumn{1}{l}{$\pi$} & (SD) \\
\hline Subspecies & & & & & & \\
Diceros bicornis minor (aggregate) & 76 & 7 & 0.267 & 0.067 & 0.0023 & 0.001 \\
$\quad$ KZN metapopulation & 65 & 1 & - & - & - & - \\
$\quad$ Zimbabwe samples & 11 & 6 & 0.855 & 0.085 & 0.0074 & 0.001 \\
D. b. michaeli & 21 & 13 & 0.952 & 0.024 & 0.0112 & 0.001 \\
D. b. bicomis & 4 & 1 & - & - & - & - \\
\hline
\end{tabular}

Table 2 MtDNA D-loop sequence variability within subspecies

Sample size (n), Number of haplotypes (H), haplotype diversity (h), Nucleotide diversity (n), Standard deviation (SD).

( $n=4)$ where only one unique haplotype was found; however, this was based on a small sample size and might not represent the total amount of genetic variation within the population. The pooled KZN samples and GenBank sequences of all D. b. minor individuals $(\mathrm{n}=79)$ contained seven haplotypes, and haplotype diversity $(h)$ was $0.267 \pm$ 0.067 and a nucleotide diversity $(\pi)$ of $0.002 \pm 0.00063$. The eight D. b. minor Zimbabwe sequences from Brown \& Houlden (2000) and two from Fernando et al. (2006) had shared haplotypes (Table 3); however, there were no shared haplotypes with the KZN samples.

The haplotype network (Fig. 2) shows a clear pattern of the separation amongst the three currently recognized subspecies with the KZN population falling out with the D. b. minor populations of Zimbabwe. Our finding of no more than three base pair substitutions between adjacent haplotypes within the D. b. michaeli subspecies is consistent with the finding by Muya et al. (2011). There is a significant separation between D. b. minor and D. $b$. bicornis with eight base pair substitutions as well as between D. b. minor and D. b. michaeli with nine base pair substitutions.

\section{Discussion}

We showed that the KZN population of D. b. minor is fixed for a single mtDNA haplotype, like most Sumatran rhino (Dicerorhinus sumatrensis) populations (Morales et al., 1997). However, unlike the Sumatran rhino populations that have occupied separate land masses for more than 10,000 years (Morales et al., 1997), it has been widely assumed that the KZN D. b. minor population has been separate from other D. b. minor populations only recently (i.e. caused by anthropogenic settlement and habitat modification during the 19th century. Swart et al., 1994). The single mtDNA haplotype in KZN
D. b. minor raises the question of whether the KZN remnant population lost genetic variation recently because of the population bottleneck or has been a genetically separate lineage for longer than previously thought.

MtDNA has a smaller effective population size $\left(\mathrm{N}_{e}\right)$ compared to nuclear loci and is one of the first genetic markers to show the genetic signature of a demographic decline. The likelihood of two or more mtDNA haplotypes persisting within an isolated population is reduced to $P<0.1$ over $4 \mathrm{~N}_{e f}$ generations, and the population is expected to become monophyletic after $4 \mathrm{~N}_{\mathrm{ef}}$ generations (Avise, Neigel \& Arnold, 1984; Mucci et al., 1999). If this holds true for the KZN D. b. minor, then recent population decline and fragmentation would have increased the rate of drift and might be responsible for the lack of haplotype diversity within the KZN black rhinoceros. Examples of monomorphic haplotypes occurring from severe bottlenecks are well documented in several species. For instance, the Whooping Crane (Grus americana) once found throughout North America had six haplotypes in ten prebottleneck museum samples, but only one haplotype persisted in the remnant postbottleneck population of 14 (Glenn, Stephan \& Braun, 1999). Such rapid declines in genetic variation have also occurred amongst southern Africa's other large mammals. For example, three small remaining remnant populations of Cape mountain zebra (Equus zebra zebra) each contain a single, unique haplotype but larger Namibian populations of closely related Hartmann's mountain zebra (E. z. hartmannae) have as many as eleven different haplotypes (Moodley \& Harley, 2006; Watson \& Chadwick, 2007). Another case in point is the loss of genetic diversity at mitochondrial and Y-chromosome loci observed in small, managed populations of Cape buffalo in Kenya and Uganda, which was attributed to restricted gene flow into protected areas (Van Hooft, Groen \& Prins, 2002). 
Appendix A

Table 3 Summary statistics for the mtDNA control region sequence variablity in each subspecies and haplotype identifiers used in Fig. 2

\begin{tabular}{|c|c|c|c|c|c|c|c|}
\hline \multirow[b]{2}{*}{ Subspecies \& population } & \multirow[b]{2}{*}{$\mathrm{n}$} & \multicolumn{6}{|c|}{ Genetic variability } \\
\hline & & $\mathrm{H}$ & h & (SD) & $\pi$ & (SD) & S \\
\hline $\begin{array}{l}\text { Dioeros bicomis minor } \\
\text { Pooled D. b. minor samples }\end{array}$ & 76 & $A-G$ & 0.267 & 0.067 & 0.002 & 0.00063 & 6 \\
\hline $\begin{array}{l}\text { Hluhluwe-iMfolozi Game Park (KZN) Accession number } \\
\text { JN593089 }\end{array}$ & 50 & A & - & - & - & - & - \\
\hline Itala Game Park (KZN) & 8 & A & - & - & - & - & - \\
\hline Mkuze Game Park (KZN) & 5 & A & - & - & - & - & - \\
\hline Ndumo Game Park (KZN) & 1 & A & - & - & - & - & - \\
\hline Johannesburg Zoo & 1 & A & - & - & - & - & - \\
\hline $\begin{array}{l}\text { Chete National Park, Zimbabwe, Accession numbers } \\
\text { AF187825-AF187827 \& AF1878329-AF1 } 87831\end{array}$ & 6 & B, C \& D & 0.733 & 0.155 & 0.004 & 0.00056 & 4 \\
\hline $\begin{array}{l}\text { Zambezi Valley, Zimbabwe, Accession numbers } \\
\text { AY742832-AY742833 }\end{array}$ & 2 & E \& G & 1 & 0.5 & 0.008 & 0.00413 & 3 \\
\hline Captive born, San Diego Zoo, Accession number AF187832 & 1 & $\mathrm{E}$ & - & - & - & - & - \\
\hline $\begin{array}{l}\text { Captive born, Westem Plains Zoo, Accession number } \\
\text { AF187828 }\end{array}$ & 1 & B & - & - & - & - & - \\
\hline Captive born, Milwaukee Zoo, Accession number AF 187833 & 1 & $\mathrm{~F}$ & - & - & - & - & - \\
\hline $\begin{array}{l}\text { D. b. michaeli } \\
\text { Pooled D. b. michaeli samples }\end{array}$ & 21 & $\mathrm{H}-\mathrm{T}$ & 0.958 & 0.0260 .011 & - & 0.00106 & 15 \\
\hline Kenya, Accession numbers FJ227483-FJ227498 & 16 & $1-s$ & 0.952 & 0.031 & 0.011 & 0.00119 & 15 \\
\hline $\begin{array}{l}\text { Solio Game Reserve, Kenya, Accession numbers, } \\
\text { AY742830-AY742831 }\end{array}$ & 2 & $\mathrm{~J}-\mathrm{T}$ & 1 & 0.5 & 0.011 & 0.00413 & 3 \\
\hline Cincinnati Zoo, Accession number AF187834 & 1 & $\mathrm{H}$ & - & - & - & - & - \\
\hline Taronga Zoo, Accession number AF 187835 & 1 & 1 & - & - & - & - & - \\
\hline $\begin{array}{l}\text { Addo Elephant Park, South Africa, Accession number } \\
\text { JN593090 }\end{array}$ & 1 & $\mathrm{~T}$ & - & - & - & - & - \\
\hline $\begin{array}{l}\text { D. b. biornis } \\
\text { Pooled D. b. bicornis samples }\end{array}$ & 4 & $\mathrm{U}$ & 0 & - & - & - & - \\
\hline Etosha, Namibia, Accession numbers JN593091-JN593094 & 4 & $\mathrm{U}$ & - & - & - & - & - \\
\hline
\end{tabular}

Sample size (n), Haplotypes, labelled A-U (H), haplotype diversity (ft), Nucleotide diversity ( $\mathrm{n})$, Standard deviation (SD), Number of segregating sites (\$).

Low genetic variation is not always a consequence of recent anthropogenic fragmentation. An alternative hypothesis is that low levels of mtDNA and autosomal variation are a result of long-term demographic separation, historically small population sizes and local adaptation. For example, despite having lower mtDNA and autosomal DNA variation, there was no evidence of a genetic bottleneck in the Yellowstone National Park, U.S. A. grizzly bear (Ursus arctos) population compared to surrounding grizzly bear populations (Miller \& Waits, 2003). Although Yellowstone's large population is embedded within the species' range, Miller \& Waits (2003) attribute the lower genetic variation to restricted gene flow into the area from the north. The common impala (Aepceros melampus melampus) of KZN also exhibited population differentiation from populations in the Limpopo Province just $490 \mathrm{~km}$ north. Schwab et al. (2012) attributed the genetic divergence to a narrow zone of unsuitable habitat below the eastern escarpment of the Drakensberg Mountains that impeded dispersal between the two provinces.

Genetic replenishment by restocking and outbreeding is recommended in cases where anthropogenically induced fragmentation has caused a loss in genetic diversity and an increase in genetic divergence. For example, 'genetic rescue' has been recommended for the Cape zebra (Moodley \& Harley, 2006; Watson \& Chadwick, 2007). However, where differences amongst genetically depauperate populations might be of natural origin, population management may need to take into account local adaptation and the possibility of outbreeding depression. 


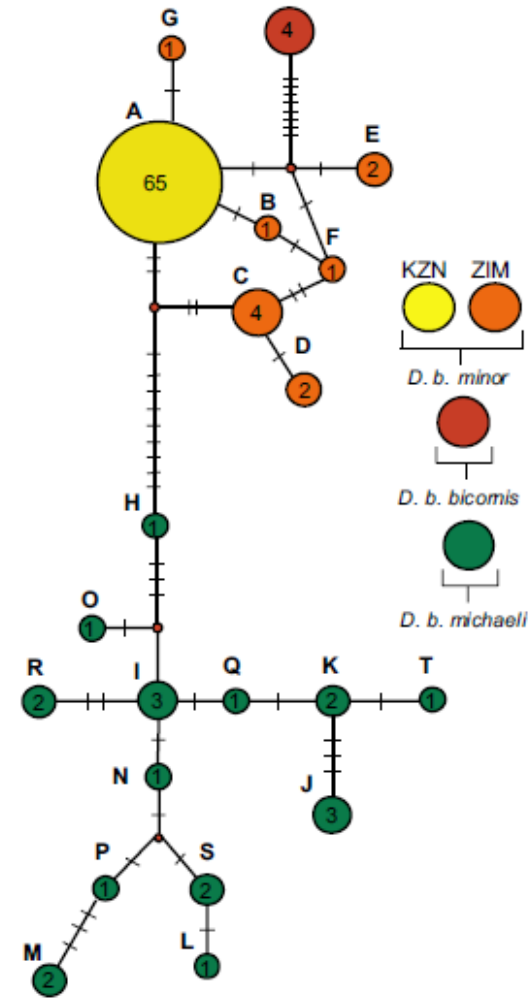

Fig 2 Statistical parsimony haplotype network calculated with Network Software for Diceros bicomis. KZN refers to the pooled D. b. minor samples within KwaZulu-Natal (KZN) (Ndumo Game Reserve, Ithala Game Reserve, Mkuze Game Reserve, HluhluweiMfolozi Game Park (HiP) and Weenan Game Reserve as well as the sample from the Johanesburg Zoo). ZIM refers to th D. b. minor samples from Zimbabwe. Circles with numbers refer to the number of sample with corresponding haplotype from Table 3. The small dots denote the mutational step, and the cross bars are the number of base pair differences.

Resolving the question regarding KZN D. b. minor mtDNA and autosomal DNA genetic structure being a recent or old event is important for guiding management plans (Rookmaaker, 2005).

Microsatellite DNA markers were previously used to assess the levels of genetic variation amongst $D$. b. minor populations. Harley et al. (2005) found appreciable amounts of variation within the $D$. b. minor subspecies using nine microsatellite loci (Table 4). They recommended that as long as heterozygosity and allele num- bers stayed at 'current' levels, no management policy change was necessary.

Based on a survey of ten microsatellites (Table 4), Karsten et al. (2011) found low levels of genetic variation within the KZN D. b. minor, but concluded that it was not cause for concern. They reached their conclusion based on (i) the similarity of allelic diversity and heterozygosity between the KZN D. b. minor population and the other subspecies; and (ii) a higher level of diversity within the black rhinoceros metapopulation compared to those found in other large African mammals. In their study, $\mathrm{H}_{\mathrm{E}}$ estimates for the D. b. bicornis and D. b. michaeli subspecies (each based on only four samples) were substantially lower than those reported by Harley et al. (2005) (Table 4). Thus, estimates for D. b. bicornis and D. b. michaeli in the study by Karsten et al. (2011) are probably underestimates. Moreover, comparisons with other large African mammals should be made cautiously. Lions in the Serengeti Plains and Ngorongoro Crater have an $\mathrm{H}_{\mathrm{E}}$ of 0.54 and 0.46 , on par with black rhinos in the study by Harley et al. (2005), yet unlike the Serengeti Plains lions the Ngorongoro Crater lions have a marked decrease in their reproductive rate attributed to inbreeding depression caused by an anthropogenic bottleneck (Brown \& Houlden, 1999; Driscoll et al. 2002).

HiP D. b. minor may be exhibiting signs of inbreeding depression owing to low reproductive rates that are not meeting the 0.25 fecundity rate desired by black rhino managers (Clinning et al, 2009). If historic gene flow is verified and inbreeding is detected, there may be a need for genetic supplementation. Supplementation would also be supported based on genetic distances between the subspecies, low levels of genetic diversity and differentiation within and amongst the KZN metapopulation reported by this mtDNA study and published autosomal microsatellite data.

The likelihood of outbreeding depression in supplemented populations of the same species is low if they have the same karyotype, have been isolated for less than 500 years and occupy similar environments (Frankham et al., 2011). Houck et al. (1995) identified the variation in chromosome morphology (number of submetacentric elements) between D. b. minor and D. b. michaeli zoo samples and recommended further studies to investigate possible differences in geographically separated populations of each subspecies in the wild. Furthermore, twenty-seven KZN D. b. minor were translocated to Malilangwe, Zimbabwe in 1997 where 
Appendix A

Table 4 Microsatellite results from Harley et al., 2005 and Karsten et al., 2011. Expected heterozygosity ( $\left.\mathrm{H}_{\mathrm{f}}\right)$, Observed heterozygosity $\left(\mathrm{H}_{0}\right)$

\begin{tabular}{|c|c|c|c|c|c|c|}
\hline Study & Microsatellites used & Subspecies & Geographic region & $\begin{array}{l}\text { Sample } \\
\text { size }\end{array}$ & $\mathrm{H}_{\mathrm{e}}$ & $\mathrm{H}_{\mathrm{o}}$ \\
\hline \multirow{3}{*}{$\begin{array}{l}\text { Harley } \\
\text { et al., } 2005\end{array}$} & BR4, BR6, BR1 7 & Diceros bicornis minor & South Africa and & 46 & 0.46 & 0.436 \\
\hline & (Cunningham et al. 1999) & D. b. bicomis & Zimbabwe & 53 & 0.51 & 0.523 \\
\hline & $\begin{array}{l}\text { DB1, DB14, DB44, DB49, DB52, DB66 } \\
\text { (Brown \& Houlden, 1999) }\end{array}$ & D. b. michaeli & $\begin{array}{l}\text { Namibia } \\
\text { South Africa }\end{array}$ & 19 & 0.68 & 0.731 \\
\hline \multirow{3}{*}{$\begin{array}{l}\text { Karsten } \\
\text { et al., 2011; }\end{array}$} & BR4, BR6, BR1 7 & D. b. minor & South Africa & 77 & 0.44 & 0.38 \\
\hline & (Cunningham et al. 1999) & D. b. biomis & Namibia & 4 & 0.43 & 0.46 \\
\hline & $\begin{array}{l}\text { DB1, DB14, DB49, DB66 } \\
\text { (Brown \& Houlden, 1999) } \\
\text { AY606078, AY606080, AY606083 } \\
\text { (Nielsen et al., 2008) }\end{array}$ & D. b. michaeli & $\begin{array}{l}\text { South Africa and } \\
\text { Tanzania }\end{array}$ & 4 & 0.54 & 0.54 \\
\hline
\end{tabular}

they were managed separately and not outbred with any Zimbabwe populations. The translocated population thrived with a growth rate of $8.3 \%$ per annum (R. du Toit, personal communication) $\mathrm{cf}$. 3.4\% over a 10 -year period (1999-2008) in HiP (Clinning et al., 2009). The success of the translocated KZN D. b. minor in Zimbabwe alleviated concerns about the adaptability of KZN rhino to Zimbabwe. The only remaining concern is whether the populations have been genetically isolated for longer than previously considered.

We recommend five research tasks to assist in resolving the genetic structure of southern Africa's black rhino as a guide to future management: (i) determine historic levels of genetic variation using museum or collection samples; (ii) investigate whether there is evidence of inbreeding depression within the HiP and KZN metapopulation; (iii) conduct a karyotype analysis on $D . b$. minor in $\mathrm{KZN}$ and Zimbabwe to determine whether chromosomal differences exist; (iv) increase the mtDNA sample size of the Zimbabwe $D$. b. minor population. Considering the high level of variation in the small sample size of the Zimbabwe sequences, a larger sample size of $D$. b. minor from that region might show that the KZN haplotype (A) is also there; and (v) lastly, genetic supplementation experiments should be implemented cautiously and systematically. A mixed population should be founded with at least 20 animals as suggested by du Toit (2006), perhaps using the Malilangwe, Zimbabwe translocation event as a template or more recent guidelines (Linklater et al., 2011. 2012). The translocated KZN D. b. minor rhinos in Malilangwe have not yet been outbred with the Zimbabwe rhinos (R. du Toit, personal communication) but might be with the F1 and F2 offspring carefully monitored for signs of reduction in reproductive fitness (outbreeding depression). If the research tasks we have recommended are completed and there is evidence of historic gene flow between KZN and Zimbabwe D. b. minor and no signs of outbreeding depression in the experimentally mixed population, then KZN D. b. minor is a candidate for genetic supplementation using progeny from Zimbabwe populations.

\section{Acknowledgements}

We thank Ezemvelo KZN Wildlife, specifically Drs Dave Cooper and Dave Druce without whom this project would not have come to fruition, the Section Rangers and Game Capture Crew at Hluhluwe-iMfolozi Park, and Dr Jacques Flamand and Pam Sheriffs of WWF's Black Rhino Range Expansion Project for their support. Thanks also to South African National Parks, specifically Michael Knight, and Ministry of Environment and Tourism, Namibia, specifically Pierre du Preez and Rudi Loutit, for facilitating earlier sampling. Thanks for financial support from the U.S. Fish and Wildife Service administered Rhinoceros and Tiger Conservation Act of 1994 (i.e. grant agreement number 96200-9-G268), including support of other projects that facilitated earlier tissue and blood sampling (e.g. grant agreement numbers 98210-2-G363, 98210-4G920 and 98210-6-G102). And a special thanks to Roan Plotz for assistance with sampling and data collecting.

\section{References}

Al.t.nnorf, F.W. \& LuikakT, G. (2007) Conservation and the Genetics of Populations. Blackwell, Malden. 
Amin, R., Thomas, K., Emst.I, R.H., Foose, T.J. \& VAn Strien, N. (2006) An overview of the conservation status of and threats to rhinoceros species in the wild. Int. Zoo Yearb. 40, 96-117.

Ashu.v, M.V., Mrinick, D.J. \& Westurn, D. (1990) Conservation genetics of the black rhinoceros (Diceros bioornis), I: Evidence from the mitochondrial DNA of three populations. Conserv. Biol. 4, 71-77.

Avise, J.C., Neigel, J.E. \& ArnotD, J. (1984) Geographic influences on mitochondrial DNA lineage survivorship in animal populations. J. Mol. Evol. 20, 99-105.

BANiet., H.J., Forster, P. \& RohI., A. (1999) Median-joining networks for inferring intraspecific phylogenies. Mol. Biol. Evol. 16, 37-48.

Brown, S.M. \& HounEN, B.A. (1999) Isolation and characterization of microsatellite markers in the black rhinoceros (Diceros bicornis). Mol. Ecol. 8, 1559-1561.

BRown, S.M. \& HoUIDEN, B.A. (2000) Conservation genetics of the black rhinoceros (Dioeros bicomis). Conserv. Gen. 1, 365-370.

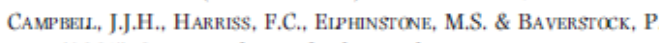
R. (1995) Outgroup heteroduplex analysis using temperature gradient gel electrophoresis - high resolution large scale screening of KNA variation in the mitochondrial control region. Mol. Eœl. Vol. 4, 407-418.

Cuinning, G., Druce, D., Robiztson, D., Bird, J. \& Nxeiz, B. (2009) Black rhino in Hluhluwe-iMfolozi Park: Historical records, status of current population and monitoring and future management recommendations. Fzemvelo KZN Wildlife, Hluhluwe.

Cunningham, J., Harley, E.H. \& O'Ryan, C. (1999) Isolation and characterization of microsatellite loci in black rhinoceros (Dixeros bicronis). Electrophoresis 20, 1778-1780.

Driscoll, C.A., Minotm-Raymond, M., Nison, G., Goldstein, D. \& O'Brien, S.J. (2002) Genomic microsatellites as evolutionary chronometers: a test in wild cats. Genome Res. 12, 414-423.

EMsin, R. (2011) Diceros bicornis. In: IUCN 2011. IUCN Red List of Threatened Species. Version 2011.2. www.iucnredlist.org. accessed on 8 February 2012.

Emsue, R., Amin, R. \& Kock, R. (2009) Guidelines for the in situ re-introduction and translocation of African and Asian rhinoceros. Occasional paper of the IUCN Species Survival Commission No. 39.

Eмsin, R. \& Broокs, M. (1999) African Rhino Status Survey and Conservation Action Plan. IUCN/SSC African Rhino Specialist Group. IUCN, Gland, Switzerland and Cambridge, UK.

Fernanid, P., Polrer, G., Fokad, N., NG, L.S., Pastorini, J. \& Meinick, D.J. (2006) Genetic Diversity, phylogeny and conservation of the Javan rhinoceros (Rhinoceros sondaicus). Conserv. Gen. 7, 439-448.

FranKham, R., BALLOU, J.D. \& BRISCOE, D.A. (2002) Introduction to Conservation Genetics, 1st edn. Cambridge University Press. Cambridge.

Frankham, R., Ballou, J.D., EIDRIIGGe, B., Lacy, R.C., Ralls, K., Dudash, M. \& Finster, C.B. (2011) Predicting the probability of outbreeding depression. Conserv. Biol. 25, 465-475.
Gl.nn, T.C., Stmphan, W. \& Braun, M.J. (1999) Effects of a population bottleneck on Whooping Crane mitochondrial DNA variation. Conserv. Biol. 13, 1097-1107.

Groves, C.P. (1964) Geographic variation in the black rhinoceros Diceros biornis (L., 1758). Z Saugetierkunde 32, 267-276.

harley, E.H., Baumgartin, I., Cunningham, J. \& O'Ryan, C. (2005) Genetic variation and population structure in remnant populations of black rhinoceros, Dioeros bicomis, in Africa. Mol. Eøol. 14, 2981-2990.

Hayashi, J.I., TAGashirA, Y. \& YoshidA, M.C. (1985) Absence of extensive recombination between inter and intraspecies mitochondrial DNA in mammalian cell. Exp. Cell Res. 160. 387-395.

Hrrchins, P.M. (1975) The black rhinoceros in South Africa. Endangered Wildlife 1, 1-2.

Hrrchins, P.M. (1984) Translocation of black rhinoceros (Diceros bicomis minor) from the Natal Game Reserves 1962-1983. The Lammergeyer 33, 45-48.

Houck, M.L., Ryder, O.A., Kumamoto, A.T. \& BinirschKe, K. (1995) Cytogenetics of the Rhinocerotidae. Proceedings of the 37th International Symposium on Disease of Zoo Animals, Dresden 25-32.

IUCN (2008) 2008 IUCN Red List of Threatened Species. IUCN, Gland, Switzerland.

KIDD, M.G. \& FrIESiN, V.L. (1998) Sequence variation in the Guillemot (Alcidae: Cepphus) mitochondrial control region and its nuclear homolog. Mol. Biol. Evol. 15, 61-70.

Karstin, M., Van VuUten, B.J., Gocoman, P. \& Barnaud, A. (2011) The history and management of black rhino in KwaZulu-Natal: a population genetic approach to assess the past and guide the future. Anim. Conserv. 14, 363-370.

Latinin, M.A., Blackshiel. Mogettigan, P.A., McWillaAm, H., Valinntin, F., Wallack, I.M., Wirm, A., Loplz, R., Thompson, J.D., Girson, T.J. \& Hricins, D.G. (2007) Clustal W and Clustal X version 2.0. Bioinformatics 23. 2947-2948.

Linkiater, W.L., Ancock, K., De Prizz, P., Swaisgood, R.R., LaW, P.R., KNIGHT, M.H., Gidir, J.V. \& KERIEY, G.I.H. (2011) Guidelines for herbivore translocation simplified: black rhinoceros case study. J. App. Ecol. 48, 493-502.

Linkiater, W.L., Gedir, J.V., Law, P.R., Swaisgood, R.R., Ancock, K., Duprezz, P., KNIGHT, M.H. \& Kerd.eY, G.I.H. (2012) Translocations as experiments in the ecological resilience of an asocial mega-herbivore. PLOS ONE 7, e30664.

MEREN.endir, A.M., WoODRUf, D.S., RYter, O.A., KoCK, R. \& VAHALA, J. (1989) Allozyme variation and differentiation in African and Indian rhinoceroses. J. Hered. 80, 377-382.

MmL.1s, C.R. \& Warts, L.P. (2003) The history of effective population size and genetic diversity in the Yellowstone grizzly (Ursus arctos): implications for conservation. PNAS 100. 43344339.

MoODL.EY, Y. \& HARIEY, E.H. (2006) Population structuring in mountain zebras (Equus zebra): the molecular consequences of divergent demographic histories. Consew. Gen. 6, 953-968. 
Appendix A

Moral.es, J.C., Andau, P.M., Supriatna, J., Zainudin, Z. \& MELNICK, D. (1997) Mitochondrial DNA variability and conservation genetics of the Sumatran rhinoceros. Conserv. Biol. 11, 539-543.

Mokrrz, C. (1994) Defining "Evolutionary Significant Units" for conservation. Trends Eøl. Evol. 9, 373-375.

Morriz, C., Dowl.ng, T.E. \& Brown, W.M. (1987) Evolution of animal mitochondrial DNA: implication for population biology and systematics. Annu. Rev. Ecol. Evol. Syst. 18, 269-292.

Moro, D., CAmpriz., J.J., Eiphinstone, M.S. \& Bravirstock, P.R. (1998) The Thevenard Island mouse: historic and conservation implications from mitochondrial DNA sequence variation. Pac. Con. Bio. 4, 282-288.

Mucc, N., Pertoldi, C., Mansen, A.B., Loischcke, V. \& Randi, E. (1999) Extremely low mitochondrial DNA control-region sequence variation in the otter Lutra lutra population of Denmark. Hereditas 130, 331-336.

MuyA, S.M. \& OGuGE, N.O. (2000) Effects of browse availability and quality on black rhinocenos (Diceros bicornis michaeli) diet in Nairobi National Park, Kenya. Afr. J. of Eøo. 38, 62-71.

Muya, S.M., Bruford, J.W., Muigal, A.W.-T., Osiemo, Z.B. MWachiro, E., OKtra-Ouma, B. \& Goossens, B. (2011) Substantial molecular variation and low genetic structure in Kenya's black rhinoceros: implications for conservation. Conserv. Gen. 12, 1575-1588.

Nibisen, L., Meghan-Mrota, D., Kilbourn, A. \& Aicivar-Warken, A. (2008) Characterization of microsatellite loci in the black rhinoceros (Diceros bicornis) and white rhinoceros (Ceratotherium simum): their use for cross-species amplification and differentiation between the two species. Conserv. Gen. 9 . 239-242.

O'Ryan, C., Flamand, J.R.B. \& HARL.EY, E.H. (1994) Mitochondrial DNA variation in black rhinoceros (Diceros bicornis): conservation management implications. Conserv. Biol. 8, 495500 .

O'Ryan, C. \& Harlex, E. (1993) Comparisons of Mitochondrial DNA in Black and White Rhinoceroses. J. Mamm. 74, 343 346.

ROOKMAAKER, L.C. (2005) The black rhino needs a taxonomic revision for sound conservation. IZN 52, 280-282.

Rozas, J., Sanchiz-Dilbarrio, J.C., Messinguir, X. \& Rozas, R. (2003) DnaSP, DNA polymorphism analyses by the coalescent and other methods. Bioinformatics 19, 2496-2497.

SAMrrook, J., Frrtsch, E.F. \& MANiATIS, T. (1989) Molecular Cloning: A Laboratory Manual. Cold Spring Harbour Laboratory Press, New York.

Schwab, P., Dizis, P.V., Wrtt, T., Haktr., G.B., Hмwe, S.S ZACHoS, F.E. \& GRoBL.ER, J.P. (2012) Genetic structure of the common impala (Aepyceros melampus melampus) in South Africa: phylogeography and implications for conservation. J. Zoo. Syst. Evol. Res. 50, 76-84.

Seutrn, G., Whrrz, B.N. \& BoAg, P.T. (1991) Preservation of avian blood and tissue samples for DNA analyses. Can. J. Zoo. 69, 82-90.

Swart, M.K.J. \& Ferguson, J.W.H. (1997) Conservation implications of genetic differentiation in southern African populations of black rhinoceros (Diceros biomis). Conserv. Biol. 11, 79-83.

Swart, M.K.J., Ferguson, J.W.H., du Tort, R. \& Flamand, J.R.B. (1994) Substantial genetic variation in Southern African black rhinoceros (Diceros bicronis). J. Hered. 850, 261-266. Sworrokd, D.L. (2002) Paup* 4.Ob: Phylogenetic Analysis Using Parsimony (*and Other Methods). Sinauer Associates. Sunderland, MA.

Tamura, K., Pertirson, D., Petzrson, N., Stechix, G., Net, G. \& KuMAR, S. (2011) MEGA5: Molecular evolutionary genetics analysis using Maximum Likelihood, Evolutionary Distance. and Maximum Parsimony methods. Mol. Biol. Evol. 28, 2731-2739.

Templ.eron, A.R. (1986) Coadaptation and outbreeding depression. In: Conservation Biology: The Science of Scarcity and Diversity (Ed. M.E. Soule). Sinauer Associates, Sunderland, MA.

Du Torr, R. (1987) The existing basis for subspecies classification of black and white rhinos. Pachyderm 9, 3-5.

DU Torr, R. (2006) Guidelines for Implementing SADC Rhino Conservation Strategies. SADC Regional Programme for Rhino Conservation, Harare, pp. 50-56.

Van Hoort, W.F., Groen, A.F. \& Prins, H.H.T. (2002) Phylogeography of the African buffalo based on mitochondria and Y-chromosomal loci: Pleistocene origin and population expansion of the Cape buffalo subspecies. Mol. Eool. 11. 267-279.

Watson, L.H. \& Chadwick, P. (2007) Management of Cape mountain zebra in the Kammanassie Nature Reserve, South Africa. S. Afr. J. Wildl. Res. 37, 31-39.

WISIERN, D. \& VIGNE, L. (1985) The deteriorating status of African rhinos. Onyx 19, 215-220.

Zukowsky, L. (1964) Die systematic der gattung Diceros Gray. 1821. Zoologische Garten, 30, 1-178.

(Mamuscript accopted 08 March 2012)

doi: 10.1111/j.1365-2028.2012.01333.x 


\section{Appendix B}

In order for harvests to be effective and to ensure that over-harvest does not occur, an accurate census in necessary. HiP black rhino managers recently reevaluated the number of $D . b$. minor in the reserve. They discovered that black rhino population estimates reported for 1998 to 2008 were over estimated (Clinning et al. 2009). Inaccurate counts during those years led to harvesting of up to $10 \%$ of the total population, which may be responsible for the low growth rate of the population (Clinning et al. 2009). Excessive harvesting can affect endangered populations that tend to already be small and more than likely have a smaller effective population size $\left(\mathrm{N}_{\mathrm{e}}\right)$ (Allendorf et al. 2008). Harvesting at levels above $10 \%$ will decrease population sizes and counteract efforts to stimulate growth (Owen-Smith 1987). HiP management has done its best to adhere to the $5 \%$ - $8 \%$ harvesting rate suggested by the SADC RMG. However, PVA modelling results suggest that managers should consider altering harvesting rates to $3 \%$ per gestational year ( $4.0 \%$ for actual year) so the population can reach its growth rate potential (e.g. higher stable population numbers and increased genetic heterozygosity; Table A.1). While the SADC RMG establishes recommended harvesting criteria for black rhino management, it must be appreciated that those guidelines are not necessarily suitable for every population. If lower harvesting rates for $\mathrm{HiP}$ was implemented $(<5 \%)$, management might see an increase a slight increase in reproductive rates.

Consideration should be taken when interpreting SADC RMG guidelines and calculating harvest regimes that not all populations are the same (e.g. different levels of heterozygosity, reproductive skew, etc.). If managers have 
Appendix B

ample and accurate life-history data on the population in question, a computer simulation should be carried out. 
Table A.1: Expected heterozygosity $\left(H_{E}\right)$ with standard error for a modelled $D$. b. minor population; with and without inbreeding at 430 carrying capacity for years $50,100,150,200,250,500,700 \&$ 1000 ; with mean population sizes and standard error. The $2 \%$ harvest is for a gestational year, which translates to $2.6 \%$ in a calendar year; $3 \%$ ( $4.0 \%$ in a calendary year); $4 \%$ ( $5.4 \%$ in a calendar year) and $5 \%(6.7 \%$. in a calendar year); $6 \%(8 \%$ in calendary year); $7 \%(9.3 \%$ in a calendar year); $8 \%(10.7 \%$ in a calendar year); $9 \%(12 \%$ in a calendary year); $10 \%(13.4 \%$ in a calendar year).

\begin{tabular}{clllll}
\hline 430 carrying capacity, 2\% Harvest & \\
\cline { 1 - 1 } & Mean Pop. & $H_{\mathrm{E}}:$ No & Mean Pop. & $\mathrm{H}_{\mathrm{E}}:$ \\
Year & Size & Inbreeding & Size & Inbreeding \\
\hline 50 & $\mathbf{3 3 4} \pm \mathbf{1 . 1 1}$ & $0.460 \pm 0.000$ & $\mathbf{3 3 1} \pm \mathbf{1 . 1 1}$ & $0.460 \pm 0.00$ \\
100 & $\mathbf{3 3 6} \pm \mathbf{1 . 1 0}$ & $0.455 \pm 0.000$ & $\mathbf{3 2 9} \pm \mathbf{1 . 1 4}$ & $0.456 \pm 0.00$ \\
150 & $\mathbf{3 3 8} \pm \mathbf{1 . 1 1}$ & $0.450 \pm 0.001$ & $\mathbf{3 2 2} \pm \mathbf{1 . 1 8}$ & $0.450 \pm 0.001$ \\
200 & $\mathbf{3 3 9} \pm \mathbf{1 . 0 6}$ & $0.446 \pm 0.001$ & $\mathbf{3 1 7} \pm \mathbf{1 . 2 1}$ & $0.446 \pm 0.001$ \\
250 & $\mathbf{3 3 9} \pm \mathbf{1 . 0 4}$ & $0.441 \pm 0.001$ & $\mathbf{3 1 5} \pm \mathbf{1 . 2 5}$ & $0.440 \pm 0.001$ \\
500 & $\mathbf{3 3 7} \pm \mathbf{1 . 0 7}$ & $0.418 \pm 0.001$ & $\mathbf{2 8 9} \pm \mathbf{1 . 3 0}$ & $0.415 \pm 0.001$ \\
700 & $\mathbf{3 3 9} \pm \mathbf{1 . 0 5}$ & $0.401 \pm 0.001$ & $\mathbf{2 7 3} \pm \mathbf{1 . 3 2}$ & $0.396 \pm 0.001$ \\
1000 & $\mathbf{3 3 7} \pm \mathbf{1 . 1 0}$ & $0.377 \pm 0.001$ & $\mathbf{2 3 6} \pm \mathbf{1 . 4 5}$ & $0.363 \pm 0.001$
\end{tabular}

\section{0 carrying capacity, $5 \%$ Harvest}

$$
H_{E} \text { : No Mean Pop. } H_{E} \text { : }
$$

Year $\sim$ Pop Size Inbreeding Size Inbreeding

$\begin{array}{llllllll}50 & \mathbf{2 3 6} \pm \mathbf{0 . 8 3} & 0.458 \pm 0.000 & \mathbf{2 3 6} \pm \mathbf{0 . 8 7} & 0.458 \pm 0.000\end{array}$

$\begin{array}{lll}100 & \mathbf{2 3 2} \pm \mathbf{0 . 7 9} 0.452 \pm 0.001 \quad 223 \pm \mathbf{0 . 7 1} & 0.451 \pm 0.001\end{array}$

$\begin{array}{llll}150 & 232 \pm 0.76 & 0.444 \pm 0.001 \quad 218 \pm 0.64 & 0.444 \pm 0.001\end{array}$

$200 \quad 231 \pm 0.77 \quad 0.437 \pm 0.001 \quad 214 \pm 0.63 \quad 0.437 \pm 0.001$

$250 \quad 231 \pm 0.750 .428 \pm 0.001211 \pm 0.670 .429 \pm 0.001$

$\begin{array}{lll}500 & \mathbf{2 3 1} \pm \mathbf{0 . 7 8} 0.394 \pm 0.0012 \mathbf{2 0 0} \pm \mathbf{0 . 7 2} 0.393 \pm 0.001\end{array}$

$1000 \mathbf{2 3 0} \pm \mathbf{0 . 7 5} 0.337 \pm 0.002 \quad \mathbf{1 5 1} \pm \mathbf{1 . 4 2} \quad 0.318 \pm 0.001$

\section{0 carrying capacity, $8 \%$ Harvest}

$$
\text { Mean Pop. } H_{E} \text { : No Mean Pop. } H_{E} \text { : }
$$

$\begin{array}{lllll}\text { Year Size } & \text { Inbreeding } & \text { Mean Pop. } & H_{E} \text { : } \\ \text { Size } & \text { Inbreeding }\end{array}$

$\begin{array}{llllll}100 & \mathbf{2 0 6} \pm \mathbf{0 . 4 2} & 0.449 \pm 0.001 & \mathbf{2 0 1} \pm \mathbf{0 . 4 6} & 0.449 \pm 0.001\end{array}$

$\begin{array}{lll}150 & \mathbf{2 0 7} \pm \mathbf{0 . 4 4} 0.441 \pm 0.001 \quad \mathbf{1 9 8} \pm \mathbf{0 . 4 9} 0.440 \pm 0.001\end{array}$

$200 \quad 206 \pm 0.44 \quad 0.432 \pm 0.001 \quad \mathbf{1 9 6}+\mathbf{0 . 5 4} 0.432 \pm 0.001$

$\begin{array}{lllllll}250 & 206 \pm 0.43 & 0.425 \pm 0.001 & \mathbf{1 9 4} \pm \mathbf{0 . 5 7} & 0.425 \pm 0.001\end{array}$

$\begin{array}{llllll}500 & \mathbf{2 0 6} \pm \mathbf{0 . 4 3} & 0.389 \pm 0.001 & \mathbf{1 8 5} \pm \mathbf{0 . 6 5} & 0.387+0.001\end{array}$

$\begin{array}{lllll}700 & 205 \pm 0.44 & 0.362 \pm 0.001 \quad 172 \pm 0.91 & 0.358 \pm 0.001\end{array}$

$1000206 \pm 0.420 .326 \pm 0.002 \quad 130 \pm 1.600 .308+0.002$

\section{0 carrying capacity, $3 \%$ Harvest}

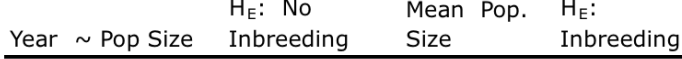

\begin{tabular}{llllll}
\hline 50 & $\mathbf{3 0 9} \pm \mathbf{1 . 2 8}$ & $0.460 \pm 0.000$ & $\mathbf{3 0 8} \pm \mathbf{1 . 2 5}$ & $0.459 \pm 0.000$
\end{tabular}

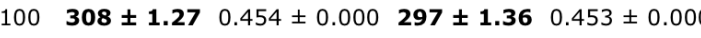

$\begin{array}{lllll}150 & \mathbf{3 1 1} \pm \mathbf{1 . 3 0} & 0.449 \pm 0.001 \quad 286 \pm \mathbf{1 3 8} & 0.448 \pm 0.001\end{array}$

$\begin{array}{llllll}200 & \mathbf{3 0 9} \pm \mathbf{1 . 2 9} & 0.444 \pm 0.001 \quad 276 \pm \mathbf{1 3 9} & 0.442 \pm 0.001\end{array}$

$250 \quad 308 \pm 1.30 \quad 0.438 \pm 0.001 \quad 267 \pm \mathbf{1 3 9} 0.435 \pm 0.001$

$500 \quad 310 \pm 1.270 .413 \pm 0.001238 \pm 1.21 \quad 0.406 \pm 0.001$

$700 \mathbf{3 0 8}+\mathbf{1 . 2 9} 0.394 \pm 0.001 \mathbf{2 1 2}+\mathbf{1 . 0 7} 0.381 \pm 0.001$

\#\#\# $\mathbf{3 0 8} \pm \mathbf{1 . 3 0} 0.366 \pm 0.001 \mathbf{1 8 0}+\mathbf{1 . 3 4} 0.341 \pm 0.002$

\section{0 carrying capacity, $6 \%$ Harvest} Size Inbreeding

$\begin{array}{llllll}50 & \mathbf{2 2 0} \pm \mathbf{0 . 6 0} & 0.457 \pm 0.000 & \mathbf{2 1 8} \pm \mathbf{0 . 5 7} & 0.457 \pm 0.00\end{array}$

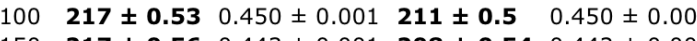
$\begin{array}{llllllll}150 & 217 \pm 0.56 & 0.443 \pm 0.001 \quad 208 \pm 0.54 & 0.442 \pm 0.001 & 0.434 & 0.001\end{array}$

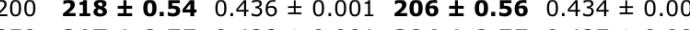
$\begin{array}{llllllll}250 & 217 & \pm 0.55 & 0.428 & 0.001 & 204 & 0.55 & 0.427 \pm 0.001\end{array}$ $\begin{array}{lllll}500 & 217 \pm 0.54 & 0.394 \pm 0.001 & 193 \pm 0.69 & 0.390 \pm 0.001\end{array}$ $\begin{array}{lllllllll}700 & 219 \pm 0.56 & 0.369 \pm 0.001 & 179 \pm 0.94 & 0.362 \pm 0.002\end{array}$

\section{0 carrying capacity, $9 \%$ Harvest}

$$
H_{E} \text { : No Mean Pop. } H_{E}
$$

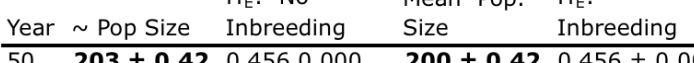

$\begin{array}{llllll}100 & \mathbf{2 0 3} \pm \mathbf{0 . 4 1} & 0.447 \pm 0.001 \quad \mathbf{1 9 8} \pm 0.45 & 0.448 \pm 0.001\end{array}$

$\begin{array}{lllllll}150 & \mathbf{2} & 03 \pm \mathbf{0 . 4 0} & 0.439 \pm 0.001 & \mathbf{1 9 5} \pm \mathbf{0 . 4 7} & 0.440 \pm 0.001\end{array}$

$200 \quad 201 \pm \mathbf{0 . 4 2} 0.431 \pm 0.001 \quad \mathbf{1 9 2} \pm 0.50 \quad 0.432 \pm 0.001$

$250 \quad 202 \pm 0.41 \quad 0.422 \pm 0.001 \quad \mathbf{1 9 1} \pm 0.59 \quad 0.424 \pm 0.001$

$500 \quad \mathbf{2 0 2}+\mathbf{0 . 4 4} 0.385+0.001 \mathbf{1 8 1} \pm \mathbf{0 . 7 4} 0.385+0.001$

$\begin{array}{llllll}700 & \mathbf{2 0 2} & \mathbf{0 . 4 2} & 0.357 \pm 0.002 & \mathbf{1 6 8} \pm \mathbf{0 . 9 8} & 0.355 \pm 0.001\end{array}$

\begin{tabular}{|c|c|c|c|c|}
\hline Year & $\begin{array}{l}\text { Mean Pop. } \\
\text { Size }\end{array}$ & $\begin{array}{l}\mathrm{H}_{\mathrm{E}}: \text { No } \\
\text { Inbreeding }\end{array}$ & $\begin{array}{l}\text { Mean Pop. } \\
\text { Size }\end{array}$ & $\begin{array}{l}\mathrm{H}_{\mathrm{E}}: \\
\text { Inbreeding }\end{array}$ \\
\hline 50 & $267 \pm 1.25$ & $0.459 \pm 0.000$ & $263 \pm 1.21$ & $0.459 \pm 0.00 c$ \\
\hline 100 & $260 \pm 1.19$ & $0.451 \pm 0.001$ & 247 & $0.453 \pm 0.001$ \\
\hline 150 & $257 \pm$ & $0.446 \pm 0.001$ & 238 & $0.447 \pm 0.001$ \\
\hline 200 & $258 \pm 1.21$ & $0.438 \pm 0.001$ & 230 & $0.440 \pm 0.001$ \\
\hline 0 & $256 \pm 1.20$ & $0.432 \pm 0.001$ & 0.90 & $0.432 \pm 0.00$ \\
\hline 500 & $259 \pm 1.23$ & $0.402 \pm 0.001$ & 0.77 & $0.397 \pm 0.001$ \\
\hline 700 & $256 \pm$ & $0.379 \pm 0.001$ & 199 & $0.370 \pm 0.001$ \\
\hline
\end{tabular}

\#\#\# $202 \pm \mathbf{0 . 4 3} 0.320 \pm 0.002 \quad \mathbf{1 2 5} \pm \mathbf{1 . 5 7} 0.307 \pm 0.002$
430 carrying capacity, 4\% Harvest

\section{0 carrying capacity, $7 \%$ Harvest}

$$
\text { Mean Pop. } H_{E} \text { : No Mean Pop. } H_{E} \text { : }
$$

Year Size Inbreeding Size Inbreeding

$\begin{array}{llllll}50 & \mathbf{2 1 3} \pm \mathbf{0 . 4 9} & 0.458 \pm 0.000 & \mathbf{2 1 0} \pm \mathbf{0 . 4 7} & 0.458 \pm 0.00 \mathrm{C}\end{array}$

$\begin{array}{lllll}211 & \pm .49 & 0.450 \pm 0.001 \quad 206 \pm 0.48 & 0.449 & 20.001\end{array}$

$\begin{array}{llll}212 & \pm .48 \quad 0.443 \pm 0.001 \quad 203 \pm 0.50 \quad 0.442 \pm 0.001\end{array}$

$\begin{array}{llll}\mathbf{2 1 0} \pm 0.50 & 0.435 \pm 0.001 \quad 200 \pm 0.55 & 0.433 \pm 0.001\end{array}$

$\begin{aligned} & 210 \pm 0.46 \quad 0.428 \pm 0.001 \\ & 197\end{aligned} 0.590 .425 \pm 0.001$

$\mathbf{2 1 1} \pm 0.490 .390 \pm 0.001 \quad 187 \pm 0.740 .388 \pm 0.001$

$\begin{array}{llll}700 & 211 \pm 0.48 & 0.364 \pm 0.001 & 176 \pm 0.91 \quad 0.360 \pm 0.00\end{array}$

\section{0 carrying capacity, $10 \%$ Harvest}

$$
\text { Mean Pop. } H_{E} \text { : No Mean Pop. } H_{E} \text { : }
$$

\begin{tabular}{lllll} 
Year & Size & Inbreeding & Size & Inbreeding \\
\hline 50 & $\mathbf{1 9 9} \pm \mathbf{0 . 3 9}$ & $0.457 \pm 0.000$ & $\mathbf{1 9 8} \pm \mathbf{0 . 4 2}$ & $0.456 \pm 0.00$
\end{tabular}

$\begin{array}{lllll}100 & \mathbf{1 9 9}+\mathbf{0 . 4 2} & 0.449 \pm 0.001 \quad \mathbf{1 9 4}+\mathbf{0 . 4 4} 0.448 \pm 0.001\end{array}$

$\begin{array}{llllll}150 & \mathbf{1 9 9} \pm \mathbf{0 . 4 1} & 0.440 \pm 0.001 & \mathbf{1 9 2} \pm \mathbf{0 . 4 9} & 0.440 \pm 0.001\end{array}$

$\begin{array}{lllll}000 & \mathbf{1 9 9} \pm \mathbf{0 . 4 0} & 0.433 \pm 0.001 & \mathbf{1 9 0} \pm \mathbf{0 . 5 1} & 0.432 \pm 0.001\end{array}$

$250 \quad \mathbf{1 9 9} \pm \mathbf{0 . 3 9} 0.424 \pm 0.001 \quad \mathbf{1 9 0} \pm \mathbf{0 . 5 1} \quad 0.423 \pm 0.001$

$\begin{array}{llllll}500 & \mathbf{1 9 9} \pm \mathbf{0 . 3 9} & 0.387 \pm 0.001 & \mathbf{1 8 0} \pm \mathbf{0 . 7 1} & 0.383+0.001\end{array}$

$\begin{array}{llllll}700 & \mathbf{1 9 9} \pm \mathbf{0 . 4 1} & 0.360 \pm 0.001 & \mathbf{1 6 5} \pm \mathbf{0 . 9 9} & 0.353 \pm 0.001\end{array}$

$1000199 \pm 0.410 .322 \pm 0.002118 \pm 1.630 .302+0.002$ 
Appendix C

\section{Appendix C}

It has already been established that cohorts consisting of individuals from HiP that are destined for reintroduction or established population supplementation within KZN have been successful as South African numbers of $D . b$. minor have steadily increased since translocations and reintroductions began in 1962 . Whether or not the success can be attributed to local adaptations however could be debated as the cohort of 27 KZN D. b. minor translocated to Mililangwe, Zimbabwe in 1997 had a higher reproductive rate than local KZN D. b. minor and no reported increases in mortality.

When running PVA computer simulations for Chapter Four, I also ran models to test the SADC RMG recommendation that cohorts used for reintroductions consist of at least 20 individuals. The results were interesting in that the cohorts with as few as 16 individuals was large enough to maintain a population from a 'numbers' standpoint (Table A2). Populations founded with 16 individuals from HiP had an $83 \%$ probability of success. The success rate increased to $88 \%$ when 20 individuals were modelled. 
Appendix C

Table A2: Probability of success or extinction for founder populations with sizes from 5- 30 individuals. The HiP results take into account the mean expected heterozygosity for each population using microsatellite DNA loc from Chapter Three with a carrying capacity of the founding reserve 50 .

\begin{tabular}{|c|c|c|c|c|c|}
\hline \multicolumn{6}{|c|}{ HiP Founders } \\
\hline $\begin{array}{l}\text { Number of } \\
\text { Individuals } \\
\text { in Starting } \\
\text { Population }\end{array}$ & $\begin{array}{l}\text { Pops. } \\
\text { that } \\
\text { Went } \\
\text { Extinct }\end{array}$ & $\begin{array}{l}\text { Pops. } \\
\text { That } \\
\text { Survived }\end{array}$ & $\begin{array}{l}\text { Probability of } \\
\text { Extinction }\end{array}$ & $\begin{array}{l}\text { Probability of } \\
\text { Success }\end{array}$ & $\begin{array}{l}\text { Mean to First } \\
\text { Extinction } \\
\text { (in years) }\end{array}$ \\
\hline 5 & 383 & 117 & $0.766 \pm 0.0189$ & $0.2340 \pm 0.0189$ & $37 \pm 3.21$ \\
\hline 6 & 331 & 169 & $0.6620 \pm 0.0212$ & $0.3380 \pm 0.0212$ & $54 \pm 4.70$ \\
\hline 7 & 297 & 203 & $0.5940 \pm 0.220$ & $0.4060 \pm 0.2200$ & $63 \pm 5.22$ \\
\hline 8 & 258 & 242 & $0.516 \pm 0.0223$ & $0.4840 \pm 0.2230$ & $57 \pm 4.63$ \\
\hline 9 & 210 & 290 & $0.4200 \pm 0.0221$ & $0.5800 \pm 0.0221$ & $88 \pm 7.44$ \\
\hline 10 & 213 & 287 & $0.4260 \pm 0.221$ & $0.5740 \pm 0.2210$ & $79 \pm 6.31$ \\
\hline 11 & 169 & 331 & $0.3380 \pm 0.0212$ & $0.6620 \pm 0.0212$ & $90 \pm 7.65$ \\
\hline 12 & 148 & 352 & $0.2960 \pm 0.0204$ & $0.7040 \pm 0.0204$ & $130 \pm 10.49$ \\
\hline 13 & 153 & 347 & $0.3060 \pm 0.0206$ & $0.6940 \pm 0.0206$ & $119 \pm 9.73$ \\
\hline 14 & 129 & 371 & $0.2580 \pm 0.0196$ & $0.7420 \pm 0.0196$ & $131 \pm 11.75$ \\
\hline 15 & 108 & 392 & $0.2160 \pm 0.0184$ & $0.7840 \pm 0.0184$ & $174 \pm 14.07$ \\
\hline 16 & 86 & 414 & $0.1720 \pm 0.0169$ & $0.8280 \pm 0.0169$ & $171 \pm 16.13$ \\
\hline 17 & 85 & 415 & $0.1700 \pm 0.0168$ & $0.8300 \pm 0.0168$ & $174 \pm 16.30$ \\
\hline 18 & 88 & 412 & $0.1760 \pm 0.0170$ & $0.8240 \pm 0.0170$ & $194 \pm 15.63$ \\
\hline 19 & 78 & 422 & $0.1560 \pm 0.0162$ & $0.8440 \pm 0.0162$ & $218 \pm 17.88$ \\
\hline 20 & 62 & 438 & $0.1240 \pm 0.0147$ & $0.8760 \pm 0.0147$ & $173 \pm 15.89$ \\
\hline 21 & 79 & 421 & $0.1580 \pm 0.0163$ & $0.8420 \pm 0.0163$ & $209 \pm 16.31$ \\
\hline 22 & 61 & 439 & $0.1220 \pm 0.0146$ & $0.8780 \pm 0.0146$ & $212 \pm 16.19$ \\
\hline 23 & 62 & 438 & $0.1240 \pm 0.0147$ & $0.8760 \pm 0.0147$ & $235 \pm 19.39$ \\
\hline 24 & 64 & 436 & $0.1280 \pm 0.0149$ & $0.8720 \pm 0.0149$ & $246 \pm 17.20$ \\
\hline 25 & 62 & 438 & $0.1240 \pm 0.0147$ & $0.8760 \pm 0.0147$ & $235 \pm 16.80$ \\
\hline 26 & 55 & 445 & $0.1100 \pm 0.0140$ & $0.8900 \pm 0.0140$ & $278 \pm 19.40$ \\
\hline 27 & 57 & 443 & $0.1140 \pm 0.0142$ & $0.8860 \pm 0.0142$ & $252 \pm 17.61$ \\
\hline 28 & 45 & 455 & $0.0900 \pm 0.0128$ & $0.9100 \pm 0.0128$ & $245 \pm 19.17$ \\
\hline 29 & 63 & 437 & $0.1260 \pm 0.0148$ & $0.8740 \pm 0.0148$ & $253 \pm 17.32$ \\
\hline 30 & 53 & 447 & $0.1060 \pm 0.0138$ & $0.8940 \pm 0.0138$ & $294 \pm 18.86$ \\
\hline
\end{tabular}


References

\subsection{References}

Adcock K (2001) Announcing the RMG carrying capacity model version 10 for black rhinos. Pachyderm 30:99-100

Adcock K, Emslie R (2003) Monitoring African rhino: an AfRSG update of

"Sandwithcarpentes" training course for field ranger, 5th edition: instructor's handbook. SADC Regional Programme for Rhino Conservation, Harare

Adcock K, Hansen HB, Lindemann H (1998) Lessons from the introduced black rhino population in Pilanesberg National Park. Pachyderm 26:40-51

Allendorf FW (1986) Genetic drift and the loss of alleles versus heterozygosity. Zoo Biol 5 (2):181-190

Allendorf FW, England PR, Luikart G, Ritchie PA, Ryman N (2008) Genetic effects of harvest on wild animal populations. Trends Ecol Evol 23 (6):327-337

Allendorf FW, Hohenlohe PA, Luikart G (2010) Genomics and the future of conservation genetics. Nat Rev Genet 11 (10):697-709

Allendorf FW, Leary RF (1986a) Heterozygosity and fitness in natural populations of animals. Conservation biology: the science of scarcity and diversity.

Sinauer Associates, Sunderland, Massachusetts

Allendorf FW, Leary RF (1986b) Heterozygosity and fitness in natural poulations of animals. In: Soule ME (ed) Conservation biology: the science of scarcity and diverstiy. Sinauer, Sunderland, Massachusetts, pp 57-76

Allendorf FW, Luikart G (2007) Conservation and the genetics of populations. Blackwell Publishing, 350 Main Street, Malden, MA U.S.A.

Amin R, Thomas K, Emslie R, Foose TJ, Van Strien N (2006) An overview of the conservation status of and threats to rhinoceros species in the wild. Int Zoo Yearb 40:96-117

Amos W, Harwood J (1998) Factors affecting levels of genetic diversity in natural populations. Phil Trans Roy Soc 353 (1366):177-186

Anderson-Lederer RM, Linklater WL, Ritchie PA (2012) Limited mitochondrial DNA variation within South Africa's black rhino (D. b. minor) population and implications for management. Afr J Ecol 50 (4):404-413

Ashley MV, Melnick DJ, Western D (1990) Conservation genetics of the black rhinoceros (Diceros bicornis), I: Evidence from the mitochondrial DNA of three popuatlions. Conserv Biol 4:71-77

Avise JC (2010) Perspective: conservation genetics enters the genomics era. Con Genet 11 (2):665-669

Avise JC, Neigel JE, Arnold J (1984) Geographic influences on mitochondrial DNA lineage survivorship in animal populations. J Mol Evol 20:99-105

Balloux F, Lugon-Moulin N (2002) The estimation of population differentiation with microsatellite markers. Molecular Ecology 11 (2):155-165

Bandelt HJ, Forster P, Rohl A (1999) Median-joining networkds for inferring intraspecific phylogenies. Mol Biol Evol 16:37-48 
Benjamini Y, Hochberg Y (1995) Controlling the False Discovery Rate - a Practical and Powerful Approach to Multiple Testing. J Roy Stat Soc B Met 57 (1):289300

Berkeley EV, Linklater WL (2010) Annual and seasonal rainfall may influence progeny sex ratio in the black rhinoceros. S Afr J Wildl Res 40 (1):53-57

Bertschinger HJ (1994) Reproduction in black and white rhinos: A review. Paper presented at the Proceedings of a Symposium on "Rhinos as Game Ranch Animals", Onderstepoort, Republic of South Africa, 9 \&10 September

Bijlsma R, Bundgaard J, Boerema AC (2000) Does inbreeding affect the extinction risk of small populations? Predictions from Drosophila. J Evolution Biol 13 (3):502-514

Bijlsma R, Loeschcke V (2012) Genetic erosion impedes adaptive responses to stressful environments. Evol Appl 5 (2):117-129

Bos DH, Gopurenko D, Williams RN, DeWoody JA (2008) Inferring population history and demography using microsatellites, mitochondrial DNA, and major histocompatibility complex (MHC) genes. Evolution 62 (6):1458-1468

Bouzat JL, Lewin HA, Paige KN (1998) The ghost of genetic diversity past: Historical DNA analysis of the greater prairie chicken. Am Nat 152 (1):1-6

Boyce MS (1992) Population Viability Analysis. Annu Rev Ecol Syst 23:481-506

Briskie JV, Mackintosh M (2004) Hatching failure increases with severity of population bottlenecks in birds. P Natl Acad Sci USA 101 (2):558-561

Brook BW, Cannon JR, Lacy RC, Mirande C, Frankham R (1999) Comparison of the population viability analysis packages GAPPS, INMAT, RAMAS and VORTEX for the whooping crane (Grus americana). Anim Conserv 2 (1):23-31

Brook BW, Lim L, Harden R, Frankham R (1997) Does population viability analysis software predict the behaviour of real populations? A retrospective study on the Lord Howe Island woodhen Tricholimnas sylvestris (Sclater). Biol Conserv 82 (2):119-128

Brook BW, O'Grady JJ, Chapman AP, Burgman MA, Akcakaya HR, Frankham R (2000) Predictive accuracy of population viability analysis in conservation biology. Nature 404 (6776):385-387

Brooks PM, Adcock K (1997) Conservation plan for the black rhinoceros Diceros bicornis in South Africa. Unpublished plan: $51 \mathrm{pp}+$ appendices

Brooks SJ (2000) Re-reading the Hluhluwe-Umfolozi Game Reserve: Contructions of a 'natural' space. Transformation (Special Issue on "Land in Africa") 24 (4):63-79

Brown SM, Houlden BA (1999) Isolation and characterization of microsatellite markers in the black rhinoceros (Diceros bicornis). Mol Ecol 8 (9):15591561

Brown SM, Houlden BA (2000) Conservation genetics of the black rhinoceros (Diceros bicornis). Con Genet 1:365-370

Bruford MW, Wayne RK (1993) Microsatellites and their application to population genetic studies. Current Opinion in Genetics \& Development 3 (6):939-943

Burger R, Lynch M (1995) Evolution and extinction in a changing environment - a quantitative-genetic analysis. Evolution 49 (1):151-163 
Campbell JJH, Harriss FC, Elphinstone MS, Baverstock PR (1995) Outgroup heteroduplex analysis using temperature gradient gel electrophoresis - high resolution large scale screening of KNA variation in the mitochondrial control region. Mol Ecol 4:407-418

Castric V, Bernatchez L, Belkhir K, Bonhomme F (2002) Heterozygote deficiencies in small lacustrine populations of brook charr Salvelinus fontinalis mitchill (Pisces, Salmonidae): a test of alternative hypotheses. Heredity 89:27-35

Caughley G (1994) Directions in conservation biology. J Anim Ecol 63 (2):215-244

Chomba C, Matandiko W (2011) Population status of black and white rhinoceros in Zambia. Pachyderm 50:50-55

Clinning G, Druce D, Robertson D, Bird J, Nxele B (2009) Black rhino in HluhluweiMfolozi Park: Historical records, status of current population and monitoring and future management recommendations. .

Coltman DW, Bowen WD, Wright JM (1998) Birth weight and neonatal survival of harbour seal pups are positively correlated with genetic variation measured by microsatellites. P Roy Soc Lond B Bio 265 (1398):803-809

Conner JC, Hartl D (2004) A primer of ecological genetics. Sinauer Associates, Inc., Sunderland, Massachusetts, U.S.A.

Conway AJ, Goodman PS (1989) Population Characteristics and Management of Black Rhinoceros Diceros-Bicornis-Minor and White Rhinoceros Ceratotherium-Simum-Simum in Ndumu-Game-Reserve, South-Africa. Biological Conservation 47 (2):109-122

Cornuet JM, Luikart G (1996) Description and power analysis of two tests for detecting recent population bottlenecks from allele frequency data. Genetics 144 (4):2001-2014

Coulson T, Mace GM, Hudson E, Possingham H (2001) The use and abuse of population viability analysis. Trends Ecol Evol 16 (5):219-221

Crawford NG (2010) SMOGD: software for the measurement of genetic diversity. Mol Ecol Resour 10 (3):556-557

Cromsigt JPGM, Hearne J, Heitkonig IMA, Prins HHT (2002) Using models in the management of black rhino populations. Ecol Model 149 (1-2):203-211

Cunningham J, Harley EH, O'Ryan C (1999) Isolation and characterization of microsatellite loci in black rhinoceros (Diceros bicornis). Electrophoresis 20 (8):1778-1780

Dinerstein E, Mccracken GF (1990) Endangered Greater One-Horned Rhinoceros Carry High-Levels of Genetic-Variation. Conserv Biol 4 (4):417-422

du Toit R (1986) Re-appraisal of black rhinoceros subspecies. Pachyderm 6:5-9

du Toit R (1987) The existing basis for subspecies classification of black and white rhinos. Pachyderm 9:3-5

du Toit R (2001) Black rhino in Lowveld Conservancies, Zimbabwe. Paper presented at the SADC Region Programme for Rhino Conservation: Proceedings of a SADC Rhino Management Group (RMG) workshop on biological management to meet continental and national black rhino conservation goals, Giants Castle, South Africa,

du Toit R (2006a) Guidelines for implementing SADC rhino conservation strategies. SADC Regional Programme for Rhino Conservation, Harare 
du Toit R (ed) (2006b) SADC Regional Programme for Rhino Conservation Strategies. SADC Region Programme for Conservation publication,

Dunn JM, Hearne J, MacArthur L (2007) A simple individual based model of black rhinoceros in Africa. Paper presented at the Preceedings of the International Congress on Modelling and Simulation, MODSIM 07, Christchurch, New Zealand, 10-13 December

Earl DA, vonHoldt BM (2012) STRUCTURE HARVESTER: a website and program for visualizing STRUCTURE output and implementing the Evanno method. Con Genet Res 4 (2):359-361

Eberhardt LL (1977) Optimal policies for conservation of large mammals with special reference to marine ecosystems. Enviro Conserv 4:205-212

Eldridge MDB, King JM, Loupis AK, Spencer PBS, Taylor AC, Pope LC, Hall GP (1999) Unprecedented low levels of genetic variation and inbreeding depression in an island population of the black-footed rock-wallaby. Conserv Biol 13 (3):531-541

Emslie R (1994) Regional conservation goals for black rhinos in Namibia/South Africa. Paper presented at the Proceedings of a symposium on rhinos as game ranch animals., Onderstepoort, South Africa, 9-10 September

Emslie R Strategic Achievement of metapopulation goals - Why rapid population growth is so important. In: Emslie R (ed) SADC Region Programme for Rhino Conservation: Proceedings of a SADC Rhino Management Group (RMG) workshop on biological management to meet continental and national black rhino conservation goals, Giants Castle, South Africa, 2001. pp 14-24

Emslie R (2011) Diceros bicornis. IUCN 2011. IUCN Red List of Threatened Species. Version $2011.2<$ www.iucnredlist.org $>$, vol 2012. IUCN,

Emslie R (2012) Ceratotherium simum. Accessed 20 October 2012

Emslie R, Brooks M (1999) African Rhino. Status Survey and Conservation Action Plan. . IUCN, Gland, Switzerland and Cambridge, UK.

Emslie R, Milledge S, Brooks M, van Strien N, Dublin T (2007) African and Asian Rhinoceroses - Status, Conservation and Trade (A report from the IUCN Survival Commission (SSC) African and Asian Rhino Specialist Groups and TRAFIC to the CITES Secretariat). vol CoP14 Doc. 54.

Emslie RH, Amin R, Kock RE (2009) Guidelines for the in situ re-introduction of African and Asian rhinoceros, vol 1. Gland, Switzerland: ICUN

England PR, Osler GHR, Woodworth LM, Montgomery ME, Briscoe DA, Frankham R (2003) Effects of intense versus diffuse population bottlenecks on microsatellite genetic diversity and evolutionary potential. Conserv Genet 4 (5):595-604

Evanno G, Regnaut S, Goudet J (2005) Detecting the number of clusters of individuals using the software STRUCTURE: a simulation study. Mol Ecol 14:2611-2620

Excoffier L, Laval G, Schneider S (2005) Arlequin (version 3.0): An integrated software package for population genetics data analysis. Evol Bioinform 1:47-50

Fanayo L, Van Rensburg S, Emslie R, Ngobese J (2005) KZN black rhino status report. . Hluhluwe iMfolozi Park: Ezemvelo KZN Wildlife, 
FANR (2010)

http://www.sadc.int/fanr/naturalresources/wildlife/programme.php. Accessed February 11, 2012

Fernando P, Polet G, Foead N, Ng LS, Pastorini J, Melnick DJ (2006) Genetic diversity, phylogeny and conservation of the Javan rhinoceros (Rhinoceros sondaicus). Conserv Genet 7 (3):439-448. doi:Doi 10.1007/S10592-0069139-4

Ferreira SM, Greaver CC, Knight MH (2011) Assessing the population performance of the black rhinoceros in Kruger National Park. South African Journal of Wildlife Research 41 (2):192-204

Foose TJ Long-term managment of small rhino populations. In: du Toit R, Foose TJ (eds) Proceedings of the 1986 Cincinnati African Rhino Workshop, 1987. Pachyderm, pp 13-15

Forstmeier W, Schielzeth H, Mueller JC, Ellegren H, Kempenaers B (2012) Heterozygosity-fitness correlations in zebra finches: microsatellite markers can be better than their reputation. Molecular Ecology 21 (13):3237-3249

Fowler CW (1981) Density Dependence as Related to Life-History Strategy. Ecology 62 (3):602-610

Fowler CW (1990) Density Dependence in Northern Fur Seals (CallorhinusUrsinus). Mar Mammal Sci 6 (3):171-195

Frankham R (1997) Do island populations have less genetic variation than mainland populations? Heredity 78:311-327

Frankham R (2005) Genetics and extinction. Biol Cons 126 (2):131-140

Frankham R, Ballou JD, Briscoe DA (2002) Introduction to conservation genetics. Cambridge University Press, Cambridge, UK

Frankham R, Ballou JD, Eldridge MDB, Lacy RC, Ralls K, Dudash MR, Fenster CB (2011) Predicting the probability of outbreeding depression. Conserv Biol 25 (3):465-475

Frankham R, Lees K, Montgomery ME, England PR, Lowe EH, Briscoe DA (1999) Do population size bottlenecks reduce evolutionary potential? Anim Conserv 2 (4):255-260

Gaggiotti OE, Lange 0, Rassmann K, Gliddon C (1999) A comparison of two indirect methods for estimating average levels of gene flow using microsatellite data. Molecular Ecology 8 (9):1513-1520

Gaillard J-M, Festa-Bianchet M, Yoccoz NG, Loison A, Toigo C (2000) Temporal variation in fitness components and population dynamics of large herbivores. Annu Rev Ecol Syst 31:367-393

Garner A, Rachlow JL, Hicks JF (2005) Patterns of genetic diversity and its loss in mammalian populations. Conserv Biol 19 (4):1215-1221

Garnier JN, Bruford MW, Goossens B (2001) Mating system and reproductive skew in the black rhinoceros. Mol Ecol 10 (8):2031-2041

Garza JC, Williamson EG (2001) Detection of reduction in population size using data from microsatellite loci. Mol Ecol 10 (2):305-318

Gibbs JP, Marquez C, Sterling EJ (2008) The role of endangered species reintroduction in ecosystem restoration: Tortoise-cactus interactions on Espanola Island, Galapagos. Restor Ecol 16 (1):88-93 
Glenn TC, Stephan W, Braun MJ (1999) Effects of a populaton bottleneck on Whooping Crane mitochondrial DNA variation. Conserv Biol 13:1097-1107

Goodman PS Black rhino harvesting strategies to improve and maintain productivity and minimise risk. In: Emslie R (ed) SADC Region Programme for Rhino Conservation: Proceedings of a SADC Rhino Management Group (RMG) workshop on biological management to meet continental and national black rhino conservation goals, Giants Castle, South Africa, 2001. pp 57-63

Goudet J (1995) FSTAT (Version 1.2): A computer program to calculate F-statistics. J Hered 86 (6):485-486

Griffith B, Scott JM, Carpenter JW, Reed C (1989) Translocation as a Species Conservation Tool - Status and Strategy. Science 245 (4917):477-480

Grobler JP, Hartl GB, Grobler N, Kotze A, Botha K, Tiedemann R (2005) The genetic status of an isolated black wildebeest (Connochaetes gnou) population from the Abe Bailey Nature Reserve, South Africa: Microsatellite data on a putative past hybridization with blue wildebeest (C-taurinus). Mamm Biol 70 (1):35-45

Grobler JP, Rushworth I, Brink JS, Bloomer P, Kotze A, Reilly B, Vrahimis S (2011) Management of hybridization in an endemic species: decision making in the face of imperfect information in the case of the black wildebeestConnochaetes gnou. Eur J Wildlife Res 57 (5):997-1006

Groves C, Grubb P (2011) Ungulate taxonomy. The Johns Hopkins University Press, Baltimore, Maryland, United States of America

Groves CP (1967) Geographic variation in the black rhinoceros Diceros gray, 1821. Zoologische Garten 30:1-178

Hall-Martin AJ, Castley JG (2001) The status of the black rhinoceros (Diceros bicornis) on private land in South Africa in 2001. Pachyderm 34:24-32

Hall-Martin AJ, Knight MH (1994) Conservation and management of black rhinoceros in South African national parks. Paper presented at the Proceedings of a symposium "Rhinos as game ranch animals", Onderstepoort,

Harley EH, Baumgarten I, Cunningham J, O'Ryan C (2005) Genetic variation and population structure in remnant populations of black rhinoceros, Diceros bicornis, in Africa. Mol Ecol 14:2981-2990

Hayashi JI, Tagashira Y, Yoshida MC (1985) Absence of extensive recombination between inter and intraspecies mitochondrial DNA in mammalian cell. Exp Cell Res 160:387-395

Hedrick PW, Parker KM, Lee RN (2001) Using microsatellite and MHC variation to identify species, ESUs, and MUs in the endangered Sonoran topminnow. Mol Ecol 10 (6):1399-1412

Hillman-Smith AKK, Groves CP (1994) Mammalian Species: Diceros bicornis. American Society of Mammalogists 455:1-8

Hitchins PM (1975) The black rhinoceros in South Africa. Endang Wildl 1:1-2

Hitchins PM (1984) Translocation of black rhinoceros (Diceros bicornis minor) from the Natal Game Reserves 1962-1983. The Lammergeyer 33:45-48 
Hogg JT, Forbes SH, Steele BM, Luikart G (2006) Genetic rescue of an insular population of large mammals. P Roy Soc Lond B Bio 273 (1593):1491-1499

Houck ML, Ryder OA, Kumamoto AT, Benirschke K Cytogenetics of the Rhinocerotidae. In: Proceedings of the 37th International Symposium on Disease of Zoo Animals

Dresden, 1995. pp 25-32

IUCN (1987) Translocation of living organisms (IUCN Position Statement). IUCN Council, Gland, Switzerland

IUCN (2008) 2008 IUCN Red List of Threatened Species. IUCN, Gland, Switzerland Jamieson IG, Wallis GP, Briskie JV (2006) Inbreeding and endangered species management: Is New Zealand out of step with the rest of the world? Conserv Biol 20 (1):38-47

Jarne P, Lagoda PJL (1996) Microsatellites, from molecules to populations and back. Trends Ecol Evol 11 (10):424-429

Johnson HE, Mills LS, Stephenson TR, Wehausen JD (2010) Population-specific vital rate contributions influence management of an endangered ungulate. Ecol Appl 20 (6):1753-1765

Jost L (2008) Gst and its relatives do not measure differentiation. Mol Ecol 17 (18):4015-4026

Jost L (2009) Population Genetics forum: topic, Calculation Jost D. vol 2012. Nature Publishing Group, http://network.nature.com/groups/popgen/forum/topics/4220,

Kalinowski ST (2005) HP-RARE 1.0: a computer program for performing rarefaction on measures of allelic richness. Mol Ecol Notes 5 (1):187-189

Karsten M, van Vuuren BJ, Goodman P, Barnaud A (2011) The history and management of black rhino in KwaZulu-Natal: a population genetic approach to assess the past and guide the future. Anim Conserv 14:363-370

Keller LF, Waller DM (2002) Inbreeding effects in wild populations. Trends Ecol Evol 17 (5):230-241

Kerley GIH, Pressey RL, Cowling RM, Boshoff AF, Sims-Castley R (2003) Options for the conservation of large and medium-sized mammals in the Cape Floristic Region hotspot, South Africa. Biological Conservation 112 (1-2):169-190

Kingdon J (1997) The Kingdon Field Guide to African Mammals. Harcourt Brace \& Company, Publishers,

Kjellander P, Hewison AJM, Liberg O, Angibault JM, Bideau E, Cargnelutti B (2004) Experimental evidence for density-dependence of home-range size in roe deer (Capreolus capreolus L.): a comparison of two long-term studies. Oecologia 139 (3):478-485

Knapp EE, Dryer AR (1998) When do genetic considerations reequire special approaches to ecological restoration? In: Fieldler PL, Karieva PM (eds) Conservation biology for the coming decade. Chapman and Hall, New York, pp 345-363

Lacombat F (ed) (2005) The evolution of the rhinoceros. . Save the Rhinos: EAZA Rhino Campaign 2005/6. European Association of Zoos and Aquaria, London 
Lacy RC (1987a) Further genetic and demographic analyses of small rhino populations. Paper presented at the Proceedings of the 1986 Cincinnati African Rhino Workshop,

Lacy RC (1987b) Loss of genetic diversity from managed populations: interacting effects of drift, mutation, immigration, selection, and population subdivision. Conserv Biol 1:143-158

Lacy RC (1993) VORTEX - a Computer-Simulation Model for Population Viability Analysis. Wildl Resource 20 (1):45-65

Lande R, Barrowclough GF (1987) Effective population size, genetic variation, and their use in population management. Viable populations for conservation. Cambridge University Press, New York

Larkin MA, Blackshields G, Brown NP, Chenna R, McGettigan PA, McWilliam H, Valentin F, Wallace IM, Wilm A, Lopez R, Thompson JD, Gibson TJ, Giggins DG (2007) Clustal W and Clustal X version 2.0. Bioinformatics 23:2947-2948

Leberg PL (1992) Effects of Population Bottlenecks on Genetic Diversity as Measured by Allozyme Electrophoresis. Evolution 46 (2):477-494

Leberg PL (1993) Stategies for population reintroduction: effects of genetic variability on population growth and size. Conserv Biol 7 (1):194-198

Leberg PL (2002) Estimating allelic richness: Effects of sample size and bottlenecks. Mol Ecol 11 (11):2445-2449

Lent PC, Fike B (2003) Home ranges, movements and spatial relationships in an expanding population of black rhinoceros in the Great Fish River Reserve, South Africa. S Afr J Wildl Res 33 (2):109-118

Lindenmayer DB, Burgman MA, Akcakaya HR, Lacy RC, Possingham HP (1995) A Review of the Generic Computer-Programs Alex, Ramas/Space and Vortex for Modeling the Viability of Wildlife Metapopulations. Ecol Model 82 (2):161-174

Lindenmayer DB, Clark TW, Lacy RC, Thomas VC (1993) Population Viability Analysis as a Tool in Wildlife Conservation Policy - with Reference to Australia. Environ Manage 17 (6):745-758

Linklater WL (2007) Translocation reverses birth sex ratio bias depending on its timing during gestation: evidence for the action of two sex-allocation mechanisms. Reprod Fert Develop 19 (7):831-839

Linklater WL, Adcock K, DePreez P, Swaisgood RR, Law PR, Knight MH, Kerley GIH (2012) Guidelines for herbivore translocation simplified: black rhinoceros case study. J Appl Ecol 48 (493-502)

Linklater WL, Gedir JV, Law PR, Swaisgood RR, Adcock K, DuPreez P, Knight MH, Kerley GIH (2011) Translocations as experiments in the ecological resilience of an asocial mega-herbivore. PLoS ONE 7 (e30664)

Lowe A, Harris S, Ashton P (2004) Ecological genetics: design, analysis and application. Blackwell Publishing, Malden, MA, U.S.A.

Lubow BC (1996) Optimal translocation strategies for enhancing stochastic metapopulation viability. Ecol Appl 6 (4):1268-1280

Luikart G, Allendorf FW, Cornuet JM, Sherwin WB (1998) Distortion of allele frequency distributions provides a test for recent population bottlenecks. J Hered 89 (3):238-247 
Luikart G, Cornuet JM (1999) Estimating the effective number of breeders from heterozygote excess in progeny. Genetics 151 (3):1211-1216

Lynch M (1996) A quantitative-genetic perspective on conservation issues. In: Avise JC, Hamrick JL (eds) Conservation genetics: case histories from nature. Chapman and Hall, New York, pp 471-501

Mace GM (2004) The role of taxonomy in species conservation. Philos T Roy Soc B 359 (1444):711-719

Manceau V, Crampe JP, Boursot P, Taberlet P (1999) Identification of evolutionary significant units in the Spanish wild goat, Capra pyrenaica (Mammalia, Artiodactyla). Anim Conserv 2 (1):33-39

Mduma SAR, Sinclair ARE, Hilborn R (1999) Food regulates the Serengeti wildebeest: a 40-year record. J Anim Ecol 68 (6):1101-1122

Merenlender AM, Woodruff DS, Ryder OA, Kock R, Vahala J (1989) Allozyme Variation and Differentiation in African and Indian Rhinoceroses. J Hered 80 (5):377-382

Messier F, Huot J, Lehenaff D, Luttich S (1988) Demography of the George River Caribou Herd - Evidence of Population Regulation by Forage Exploitation and Range Expansion. Arctic 41 (4):279-287

Miller BJ, Anderson SH, Doncarlos MW, Thorne ET (1988) Biology of the Endangered Black-Footed Ferret and the Role of Captive Propagation in Its Conservation. Can J Zool 66 (4):765-773

Miller CR, Waits LP (2003) The history of effective population size and genetic diversity in the Yellowstone grizzly (Ursus arctos): implication for conservation. PNAS 100:4334-4339

Miller PS, Lacy RC (2005) VORTEX: A stochastic simulation of the Extinction Process. Version 9.5 User's Manual. Conservation Breeding Specialist Group (SSC/IUCN), Apple Valley, MN

Mills A, Morkel P, Amiyo A, Runyoro V, Borner M, Thirgood S (2006) Managing small populations in practice: black rhino Diceros bicornis michaeli in the Ngorongoro Crater, Tanzania. Oryx 40 (3):319-323

Moehlman PD, Amato G, Runyoro V (1996) Genetic and demographic threats to the black rhinoceros population in the Ngorongoro Crater. Conserv Biol 10 (4):1107-1114

Moodley Y, Harley EH (2005) Population structuring in mountain zebras (Equus zebra): The molecular consequnces of divergent demographic histories. Con Genet 6:953-968

Morales JC, Andau PM, Supriantna J, Zainuddin Z, Melnick D (1997) Mitochondrial DNA variability and conservation genetics of the Sumatran rhinoceros. Conserv Biol 11:539-543

Moritz C (1994) "Evolutionary Significant Units" for conservation. Trends Ecol Evol 9:373-375

Moritz C (2002) Strategies to protect biological diversity and the evolutionary processes that sustain it. Syst Biol 51 (2):238-254

Moritz C, Dowling TE, Brown WM (1987) Evolution of animal mitochondrial DNA: implicaton for population biology and systematics. Annu Rev Ecol Evol Syst 18:269-292 
Moro D, Campbell JJ, Elphinstone MS, Braverstock PR (1998) The Thevendard Island mouse: Historic and conservation implications for mitochondrial DNA sequence variation. Pac Conserv Biol 4:282-288

Mucci N, Pertoldi C, Madsen AB, Loeschcke V, Randi E (1999) Extremely low mitochondrial DNA control-region sequence variation in the other Lutra lutra populatons of Denmark. Hereditas 130:331-336

Muya SM, Bruford JW, Muigai AW-T, Osiemo ZB, Mwachiro E, Okita-Ouma B, Goossens B (2011) Substantial molecular variation and low genetic structure in Kenya's black rhinoceros: implications for conservation. Conserv Genet 12:1575-1588

Muya SM, Oguge NO (2000) Effects of browse availability and quality on black rhinoceros (Diceros bicornis michaeli) diet in Nairobi National Park, Kenya. Afr J Ecol 38:62-71

Nei M (1978) Estimation of average heterozygosity and genetic distance from a small number of individuals. Genetics 89 (3):583-590

Nei M, Marayuma T, Chakraaborty R (1975) The bottleneck effect and genetic variability in populations. Evolution 29:1-10

Nelson NJ, Keall SN, Brown D, Daugherty CH (2002) Establishing a new wild population of tuatara (Sphenodon guntheri ). Conserv Biol 16 (4):887-894

Nielsen L, Meehan-Meola D, Kilbourn A, Alcivar-Warren A (2008) Characterization of microsatellite loci in the black rhinoceros (Diceros bicornis) and white rhinoceros (Ceratotherium simum): their use for cross species amplification and differentiation between the two species. Conserv Genet 9:239-242

O'Ryan C, Flamand JRB, Harley EH (1994) Mitochondrial DNA variation in black rhinoceros (Diceros bicornis): Conservation management implications. Conserv Biol 8:495-500

O'Ryan C, Harley EH (1993) Comparisons of mitochondrial DNA in black and white rhinoceroses. J Mammal 74:343-346

Ouborg NJ, Pertoldi C, Loeschcke V, Bijlsma R, Hedrick PW (2010) Conservation genetics in transition to conservation genomics. Trends Genet 26 (4):177187

Owen-Smith N (1987) Pleistocene extinctions: The pivotal role of megaherbivores. Paleonto Soc 13 (3):351-362

Owen-Smith RN (1988) Megaherbivores: the influence of very large body size on ecology. University Press, Cambridge

Owen-Smith RN Overview of the population dynamics of large mammals. In: Emslie R (ed) SADC Region Programme for Rhino Conservation: Proceedings of a SADC Rhino Management Group (RMG) workshop on biological management to meet continental and national black rhino conservation goals, Giants Castle, South Africa, 2001. pp 9-13

Palstra FP, Ruzzante DE (2008) Genetic estimates of contemporary effective population size: what can they tell us about the importance of genetic stochasticity for wild population persistence? Mol Ecol 17 (15):3428-3447

Pemberton JM, Slate J, Bancroft DR, Barrett JA (1995) Nonamplifying Alleles at Microsatellite Loci - a Caution for Parentage and Population Studies. Mol Ecol 4 (2):249-252 
Piertney SB, Oliver MK (2006) The evolutionary ecology of the major histocompatibility complex. Heredity 96 (1):7-21

Piry S, Luikart G, Cornuet JM (1999) BOTTLENECK: A computer program for detecting recent reductions in the effective population size using allele frequency data. J Hered 90 (4):502-503

Possingham HP, Lindenmayer DB, Norton TW (1993) A framework for improved threatened species management using Population Viability Analysis. Pac Conserv Biol 1:39-45

Pritchard JK, Stephens M, Donnelly P (2000) Inference of population structure using multilocus genotype data. Genetics 155 (2):945-959

Pritchard JK, Wen X, Falush D (2007) Documentation for structure software: Version 2.2. http://pritch.bsd.uchicago.edu/doftware/structure22/readme.pdf

Ralls K, Ballou JD, Templeton AR (1988) Estimates of lethal equivalents and the cost of inbreeding in mammals. Conserv Biol 2:185-193

Raymond M, Rousset F (1995) Genepop (Version-1.2) - Population-Genetics Software for Exact Tests and Ecumenicism. J Hered 86 (3):248-249

Robertson HA, Karika I, Saul EK (2006) Translocation of Rarotonga Monarchs Pomarea dimidiata within the souther Cook Islands. Bird Conserv Internat 16:197-215

Rodriguez-Ramilo ST, Wang JL (2012) The effect of close relatives on unsupervised Bayesian clustering algorithms in population genetic structure analysis. Mol Ecol Resour 12 (5):873-884

Rookmaaker LC (1995) Subspecies and ecotypes of the black rhinoceros. Pachyderm 20:39-40

Rookmaaker LC (2000) The alleged population reduction of the southern white rhinoceros (Ceratotherium simum simum) and the successful recovery. Säugetierkundliche Mitteilungen 45 (2):54-70

Rookmaaker LC (2004) Historical distribution of the black rhinoceros (Diceros bicornis) in West Africa. Afr Zoo 39 (1):63-70

Rookmaaker LC (2005) The black rhino needs a taxonomic revision for sound conservation. Int Zoo Yearb 52 (5):280-282

Rookmaaker LC (2007) A chronological survey of bibliographical and iconographical sources on rhinoceroses in southern Africa from 1795 to 1875: reconstructing views on classification and changes in distribution. Trans Roy Soc SAfr 62 (2):55-198

Rookmaaker LC (2011) A review of black rhino systematics proposed in Ungulate Taxonomy by Groves and Brubb (2011) and its implication for rhino conservation. . Pachyderm 50:72-76

Rousset F (2008) GENEPOP ' 007: a complete re-implementation of the GENEPOP software for Windows and Linux. Mol Ecol Resour 8 (1):103-106

Roy MS, Girman DJ, Taylor AC, Wayne RK (1994) The Use of Museum Specimens to Reconstruct the Genetic-Variability and Relationships of Extinct Populations. Experientia 50 (6):551-557 
Rozas J, Sanches-DelBarrio JC, Messenguer X, Rozas R (2003) DnaSP, DNA polymorphism analyses by the coalescent and other methods.

Bioinformatics 19 (2496-2497)

Rubinoff D, Holland BS (2005) Between two extremes: Mitochondrial DNA is neither the panacea nor the nemesis of phylogenetic and taxonomic inference. Systematic Biol 54 (6):952-961

Sambrook J, Fritsch EF, Maniatis T (1989) Molecular cloning: A laboratory manual. Cold Spring Harbour Laboratory Press, New York

Schenkel R, Schenkel-Hulliger L (1969) Ecology and behaviour of the black rhinoceros (Diceros bicornis L.). Mammalia Depicta (series). Verlag Paul Parey Scientific Publishers, Hamburg and Berlin

Schuelke M (2000) An economic method for the fluorescent labeling of PCR fragments. Nat Biotechnol 18 (2):233-234

Schwab P, Debes PV, Witt T, Hartl GB, Hmwe SS, Zachos FE, Grobler JP (2012) Genetic structure of the common impala (Aepyceros melampus melampus) in South Africa: phylogeography and implications for conservation. J Zool Syst Evol Res 50 (1):76-84

Schwartz MK, Luikart G, Waples RS (2006) Genetic monitoring as a promising tool for conservation and management. Trends Ecol Evol 22 (1):25-33

Selander RK, Johnson WE (1973) Genetic variation among vertebrate species. Annual Review of Ecology and Systematics 4:75-91

Seutin G, White BN, Boag PT (1991) Preservation of avian blood and tissue samples for DNA analyses. Can J Zoo 69 (82-90)

Shaffer HB, Fisher RN, Davidson C (1998) The role of natural history collections in documenting species declines. Trends Ecol Evol 13 (1):27-30

Shaffer ML (1990) Population Viability Analysis - Comment. Conserv Biol 4 (1):3940

Shoo LP, Williams SE, Hero J (2006) Detecting climate change induced range shifts: Where and how should we be looking? Austral Ecology 31 (1):22-29

Silberman MS, Fulton RB (1979) Medical Problems of Captive and Wild Rhinoceros - Review of the Literature and Personal Experiences. J Zoo Anim Med 10 (1):6-16

Simonsen BT, Siegismund HR, Arctander P (1998) Population structure of African buffalo inferred from mtDNA sequences and microsatellite loci: high variation but low differentiation. Mol Ecol 7 (2):225-237

Singer FJ, Papouchis CM, Symonds KK (2000) Translocations as a tool for restoring populations of bighorn sheep. Restor Ecol 8 (4):6-13

Skinner JD, Smithers RHN (1990) The mammals of the southern Africa subregion, new edition. University of Pretoria, Pretoria

Skogland T (1985) The Effects of Density-Dependent Resource Limitations on the Demography of Wild Reindeer. J Anim Ecol 54 (2):359-374

Slatkin M (1995) A Measure of Population Subdivision Based on Microsatellite Allele Frequencies (Vol 139, Pg 457, 1995). Genetics 139 (3):1463-1463

Smith KG, Clark JD (1994) Black Bears in Arkansas - Characteristics of a Successful Translocation. J Mammal 75 (2):309-320 
Sommer S (2003) Effects of habitat fragmentation and changes of dispersal behaviour after a recent population decline on the genetic variability of noncoding and coding DNA of a monogamous Malagasy rodent. Molecular Ecology 12 (10):2845-2851

Soulé ME (1980) Thresholds for survival: maintaining fitness and evolutionary potential. Conservation biology: an evolutionary-ecological perspective. Sinauer, Sunderland, Massachusetts

Strand TM (2011) Genotyping of black grouse MHC class II B using reference strand-mediated conformational analysis (RSCA). BMC Research Notes $4: 183$

Swart J, Goodman P, Hearne JW A conservation model for black rhino. In: Anderson DF, Richardson GP, Sterman JD (eds) The 8th International Conference of the Sytem Dynamics Society 1990, Chestnut Hill, Massachusetts, U.S.A., 1990. pp 1128-1136

Swart MKJ, Ferguson JWH (1997) Conservation implications of genetic differentiation in southern African populations of black rhinoceros (Diceros bicornis). Conserv Biol 11 (1):79-83

Swart MKJ, Ferguson JWH, Dutoit R, Flamand JRB (1994) Substantial GeneticVariation in Southern African Black Rhinoceros (Diceros bicornis). J Hered 85 (4):261-266

Swofford DL (2002) PAUP* 4.0b: Phylogenetic analysis using parsimony (*and other methods). Sinauer Associates, Sunderland, MA

Taberlet P, Bouvet J (1994) Mitochondrial-DNA Polymorphism, Phylogeography, and Conservation Genetics of the Brown Bear Ursus arctos in Europe. P Roy Soc Lond B Bio 255 (1344):195-200

Tacha TC, Warde WD, Burnham DP (1982) Use and interretation of statistics in wildlife journals. Wildlife Society Bulletin 10 (4):355-362

Talukdar BK, Emslie R, Bist SS, Choudhury A, Ellis S, Bonal BS, Malakar MC, Talukdar BN, Barua M (2008) Rhinoceros unicornis. Accessed 06 March 2013

Tamura K, Peterson D, Peterson N, Stecher G, Nei M, Kumar S (2011) MEGA5: Molecular Evolutionary Genetics Analysis Using Maximum Likelihood, Evolutionary Distance, and Maximum Parsimony Methods. Mol Biol Evol 28 (10):2731-2739. doi:Doi 10.1093/Molbev/Msr121

Templeton AR (ed) (1986) Coadaptation and outbreeding depression. Conservation Biology. Sinauer Associates, Sunderland, MA

Tenhumberg B, Tyre AJ, Shea K, Possingham HP (2004) Linking wild and captive populations to maximize species persistence: Optimal translocation strategies. Conserv Biol 18 (5):1304-1314

Tews J, Ferguson MAD, Fahrig L (2007) Modeling density dependence and climatic disturbances in caribou: a case study from the Bathurst Island complex, Canadian High Arctic. J Zool 272 (2):209-217

Tingley MW, Beissinger SR (2009) Detecting range shifts from historical species occurrences: new perspectives on old data. Trends Ecol Evol 24 (11):625633. doi:Doi 10.1016/J.Tree.2009.05.009

Toews DPL, Brelsford A (2012) The biogeography of mitochondrial and nuclear discordance in animals. Mol Ecol 21:3907-3930 
Tougard C, Delefosse T, Hanni C, Montgelard C (2001) Phylogenetic relationships of the five extant rhinoceros species (Rhinocerotidae, Perissodactyla) based on mitochondrial cytochrome b and 12S rRNA genes. Mol Phylogenet Evol 19 (1):34-44. doi:Doi 10.1006/Mpev.2000.0903

Tracy LN, Wallis GP, Efford MG, Jamieson IG (2011) Preserving genetic diversity in threatened species reintroductions: how many individuals should be released? Anim Conserv 14 (4):439-446

Traill LW, Brook BW, Frankham RR, Bradshaw CJA (2010) Pragmatic population viability targets in a rapidly changing world. Biol Conserv 143 (1):28-34

Tyus HM, Saunders JF (2000) Nonnative fish control and endangered fish recovery: Lessons from the Colorado River. Fisheries 25 (9):17-24

Van Coeverden de Groot PJ, Putnam AS, Erb P, Scott C, Melnick D, O'Ryan C, Boag PT (2011) Conservation genetics of the black rhinoceros, Diceros bicornis bicornis, in Namibia. Con Genet 12:783-792

Van Dierendonck MC, Wallis de Vries MF (1996) Ungulate reintroductions: experiences with the Takhi or Przewalski Horse (Equus ferus przewalskii) in Mongolia. Conserv Biol 10 (3):728-740

van Hooft WF, Groen AF, Prins HHT (2000) Microsatellite analysis of genetic diversity in African buffalo (Syncerus caffer) populations throughout Africa. Molecular Ecology 9 (12):2017-2025

Van Hooft WF, Groen AF, Prins HHT (2002) Phylogeography of the African buffalo based on mitochondrial and Y-chromosomal loci: Pleistocene origin and population expansion of the Cape buffalo subspecies. Mol Ecol 11:267-279

Van Houtan KS, Halley JM, van Aarde R, Pimm SL (2009) Achieving success with small, translocated mammal populations. Conserv Lett 2 (6):254-262

van Strien NJ, Manullang B, Sectionov, Isnan W, Khan MKM, Sumardja E, Ellis S, Han KH, Boeadi, Payne J, Martin B (2008a) Dicerorhinus sumatrensis. IUCN 2012. IUCN Red List of Threatened Species. Version 2012.2 $<$ www.iucnredlist.org $>$. Accessed 20 October 2012

van Strien NJ, Steinmetz R, Manullang B, Sectionov, Han KH, Isnan W, Rookmaaker K, Sumardja E, Khan MKM, Ellis S (2008b) Rhinoceros sondaicus. IUCN 2012. IUCN Red List of Threatened Species. Version 2012.2 $<$ www.iucnredlist.org $>$. Accessed 20 October 2012

Vila C, Sundqvist AK, Flagstad O, Seddon J, Bjornerfeldt S, Kojola I, Casulli A, Sand H, Wabakken P, Ellegren H (2003) Rescue of a severely bottlenecked wolf (Canis lupus) population by a single immigrant. P Roy Soc Lond B Bio 270 (1510):91-97

Waite TA, Vucetich J, Saurer T, Kroninger M, Vaughn E, Field K, Ibargüen S (2005) Minimizing extinction risk through genetic rescue. Anim Biodiv Conserv 28.2:121-130

Waits L, Taberlet P, Swenson JE, Sandegren F, Franzen R (2000) Nuclear DNA microsatellite analysis of genetic diversity and gene flow in the Scandinavian brown bear (Ursus arctos). Mol Ecol 9 (4):421-431

Waits LP, Talbot SL, Ward RH, Shields GF (1998) Mitochondrial DNA phylogeography of the North American brown bear and implications for conservation. Conserv Biol 12 (2):408-417 
Walters JR (1991) Application of Ecological Principles to the Management of Endangered Species - the Case of the Red-Cockaded Woodpecker. Annu Rev Ecol Syst 22:505-523

Watson LH, Chadwick P (2007) Management of Cape mountain zebra in the Kammanassie Nature Reserve, South Africa. S Afr J Wildl Res 37 (1):31-39

Weckworth BV, Musiani M, McDevitt AD, Hebblewhite M, Mariani S (2012) Reconstruction of caribou evolutionary history in Western North America and its implications for conservation. Mol Ecol 21 (14):3610-3624

Westemeier RL, Brawn JD, Simpson SA, Esker TL, Jansen RW, Walk JW, Kershner EL, Bouzat JL, Paige KN (1998) Tracking the long-term decline and recovery of an isolated population. Science 282 (5394):1695-1698

Western D, Vigne L (1985) The deteriorating status of African rhinos. Oryx 19:215220

Whitehouse AM, Harley EH (2001) Post-bottleneck genetic diversity of elephant populations in South Africa, revealed using microsatellite analysis. Molecular Ecology 10 (9):2139-2149

Wright S (1965) The Interpretation of Population-Structure by F-Statistics with Special Regard to Systems of Mating. Evolution 19 (3):395-420

Yamamoto S, Maekawa K, Tamate T, Koizumi I, Hasegawa K, Kubota H (2006) Genetic evaluation of translocation in artificially isolated populations of white-spotted charr (Salvelinus leucomaenis). Fish Res 78 (2-3):352-358

Zenger KR, Richardson BJ, Vachot-Griffin AM (2003) A rapid population expansion retains genetic diversity within European rabbits in Australia. Mol Ecol 12 (3):789-794

Zukowsky L (1965) Die systematic der gattung Diceros Gray, 1821. Zoologische Garten 30:1-178 